\title{
Modeling asymmetric and time-varying dependence
}

Citation for published version (APA):

Manner, H. (2010). Modeling asymmetric and time-varying dependence. [Doctoral Thesis, Maastricht University]. Datawyse / Universitaire Pers Maastricht. https://doi.org/10.26481/dis.20100318hm

Document status and date:

Published: 01/01/2010

DOI:

10.26481/dis.20100318hm

Document Version:

Publisher's PDF, also known as Version of record

\section{Please check the document version of this publication:}

- A submitted manuscript is the version of the article upon submission and before peer-review. There can be important differences between the submitted version and the official published version of record.

People interested in the research are advised to contact the author for the final version of the publication, or visit the DOI to the publisher's website.

- The final author version and the galley proof are versions of the publication after peer review.

- The final published version features the final layout of the paper including the volume, issue and page numbers.

Link to publication

\footnotetext{
General rights rights.

- You may freely distribute the URL identifying the publication in the public portal. please follow below link for the End User Agreement:

www.umlib.nl/taverne-license

Take down policy

If you believe that this document breaches copyright please contact us at:

repository@maastrichtuniversity.nl

providing details and we will investigate your claim.
}

Copyright and moral rights for the publications made accessible in the public portal are retained by the authors and/or other copyright owners and it is a condition of accessing publications that users recognise and abide by the legal requirements associated with these

- Users may download and print one copy of any publication from the public portal for the purpose of private study or research.

- You may not further distribute the material or use it for any profit-making activity or commercial gain

If the publication is distributed under the terms of Article $25 \mathrm{fa}$ of the Dutch Copyright Act, indicated by the "Taverne" license above, 
Modeling Asymmetric

and

Time-Varying Dependence

Hans Manner 
(C) Hans Manner, 2010

All rights reserved. No part of this publication may be reproduced, stored in a retrieval system, or transmitted in any form, or by any means, electronic, mechanical, photocopying, recording or otherwise, without the prior permission in writing from the author.

This book was typeset by the author using $\mathrm{LT}_{\mathrm{E}} \mathrm{X}$.

Published by Universitaire Pers Maastricht

ISBN: 978-90-5278-922-4

Printed in The Netherlands by Datawyse Maastricht 


\title{
Modeling Asymmetric
}

\author{
and \\ Time-Varying Dependence
}

PROEFSCHRIFT

ter verkrijging van de graad van doctor

aan de Universiteit Maastricht,

op gezag van de Rector Magnificus,

Prof. mr. G.P.M.F. Mols,

volgens het besluit van het College van Decanen,

in het openbaar te verdedigen op

donderdag 18 maart 2010 om 16.00 uur

door

Hans Manner 


\section{Promotores:}

Prof. dr. F.C. Palm

Prof. dr. J.R.Y.J Urbain

\section{Beoordelingscommissie:}

Prof. dr. P. Schotman (voorzitter)

Prof. dr. A. Monfort

Dr. A. J. Patton (Duke University, Durham, USA)

Dit onderzoek werd gefinancieerd door de Maastricht Research School of Economics of Technology and Organizations (METEOR). 


\section{Acknowledgements}

Many people have contributed to this thesis in ones way or another and helped me shape it the way it is. First of all I want to thank my supervisors Franz Palm and Jean-Pierre Urbain, who supported me greatly in my professional development, but also took an honest interest in my private life, which I highly appreciate. I thank the members of the Degree Committee, Peter Schotman, Alain Monfort and Andrew Patton, for taking the time to read and referee this thesis. I also want to thank my co-authors Bertrand Candelon, Christian Hafner, Johan Segers and Olga Reznikova for pleasant collaborations and for teaching me a lot. Next, I must mention Alain Hecq who woke my interest in copulas and supervised my master thesis on that topic, which turned into the first chapter of this book. Many thanks to my colleagues at the Department of Quantitative Economics for providing an enjoyable working environment, to Karin van den Boorn and Haydeé Hallmanns for helping me with those many little organizational things and to Robert Vermeulen for helping me with the dutch summary. I also express my gratitude to the lunch gang consisting of Christian Gengenbach, Sascha Wolf, Christoph Hanck, Wladimir Raymond and, towards the end, Dennis Tuerk for both interesting and sometimes extremely absurd conversations and for being nice company. Thanks also to Giovanni Motta not only for enjoyable lunches that were not spent with the above mentioned people, but also for introducing me three quarters of my co-authors and helping me organize my stay in Louvain-laNeuve.

Most importantly, I want to thank my parents Ruth and Dietmar Manner and my brother Paul for their great support over the years and for helping me develop into the person I am today. Finally, I want to express my greatest love and gratitude to my wife Mayra and my son Henry for making even the most stressful times a pleasure to live. Thank you for always being there for me and for 
being such a warm, loving and funny family.

Hans Manner

Maastricht, January 2010 


\section{Contents}

Acknowledgements $\quad$ v

1 Introduction $\quad 1$

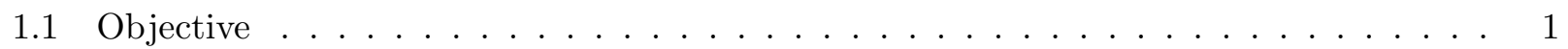

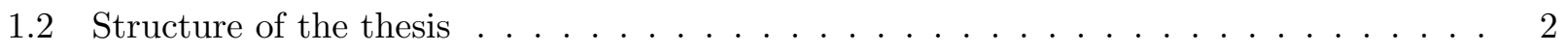

2 Estimation and model selection of copulas with an application to exchange rates 5

2.1 Introduction . . . . . . . . . . . . . . . . . . . . . . . . . 5

2.2 Introducing copulas and related concepts $\ldots \ldots \ldots \ldots \ldots$

$2.2 .1 \quad$ Preliminaries and copulas defined $\ldots \ldots \ldots \ldots \ldots \ldots$

2.2 .2 Examples and families of copulas . . . . . . . . . . . . . . . 11

2.2 .3 Simulation from a copula . . . . . . . . . . . . . . . . 15

2.3 Estimation and model selection . . . . . . . . . . . . . . . . . . 20

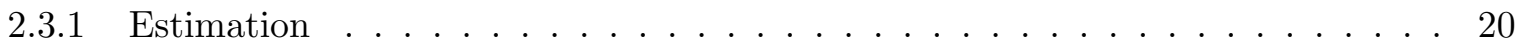

2.3 .2 Model selection . . . . . . . . . . . . . . . . . . . . . . . . . . . 24

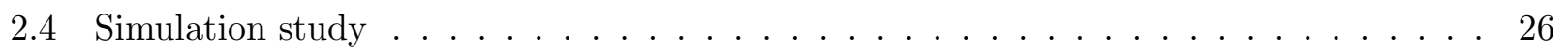

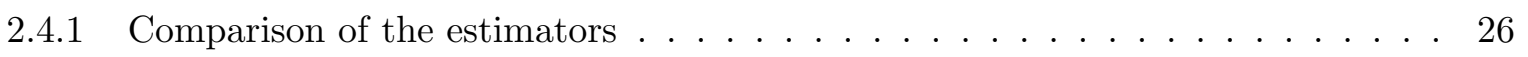

2.4 .2 Model selection . . . . . . . . . . . . . . . . . . . . 28

2.5 Modeling exchange rate dependence $\ldots \ldots \ldots \ldots$

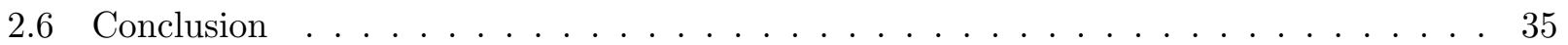

$\begin{array}{lll}3 & \text { Testing for asymmetric dependence } & 37\end{array}$

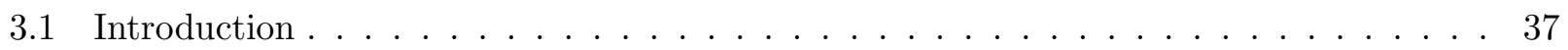


3.2 Testing for asymmetric dependence . . . . . . . . . . . . . . . . . . . 39

3.2 .1 Asymmetric dependence . . . . . . . . . . . . . . . . . . 39

$3.2 .2 \quad$ A test based on mixtures of copulas . . . . . . . . . . . . . . . . 41

$3.2 .3 \quad$ Local power . . . . . . . . . . . . . . . . . . . . . . . . 47

3.3 How asymmetry is generated $\ldots \ldots \ldots \ldots \ldots \ldots$

3.4 Monte Carlo studies . . . . . . . . . . . . . . . . . . . . . 54

3.4 .1 The general case . . . . . . . . . . . . . . . 54

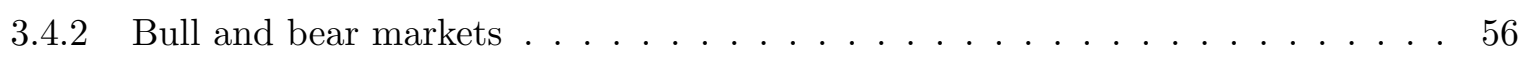

3.4 .3 Nonlinear VAR . . . . . . . . . . . . . . . . . . 57

3.4.4 SETAR models . . . . . . . . . . . . . . . . . . . . 57

3.5 Empirical applications $\ldots \ldots \ldots \ldots \ldots \ldots \ldots \ldots$

3.5.1 International stock market returns . . . . . . . . . . . . . . . . 59

3.5.2 Application on US output and unemployment data . . . . . . . . . . . . 60

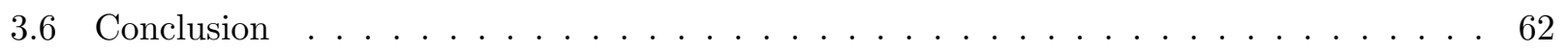

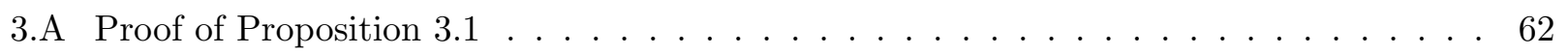

4 Time-varying copulas: a survey $\quad 65$

4.1 Introduction . . . . . . . . . . . . . . . . . . . . 65

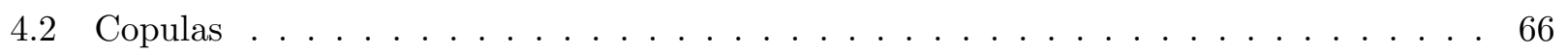

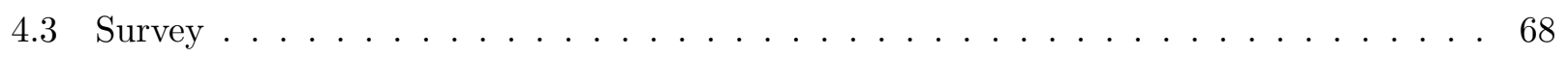

4.3 .1 Observation driven models $\ldots \ldots \ldots \ldots \ldots$

$4.3 .2 \quad$ DCC copulas . . . . . . . . . . . . . . . . . . . . 71

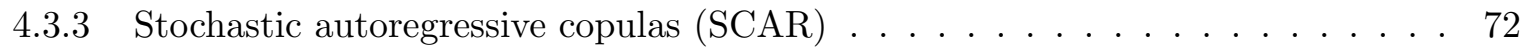

4.3.4 Semiparametric dynamic copula $(\mathrm{SDC}) \ldots \ldots \ldots \ldots$. . . . . . . . 73

4.3 .5 Structural breaks . . . . . . . . . . . . . . . . . . . . . 74

4.3 .6 Adaptive estimation method $(\mathrm{LCP}) \ldots \ldots \ldots \ldots \ldots$

4.3 .7 Other approaches . . . . . . . . . . . . . . 77

4.4 Model selection and simulations . . . . . . . . . . . . . . . . 78

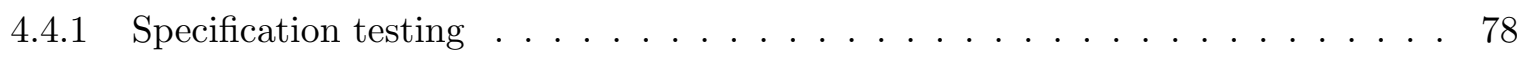

4.4 .2 Monte Carlo study . . . . . . . . . . . . . . . . . . . . 79

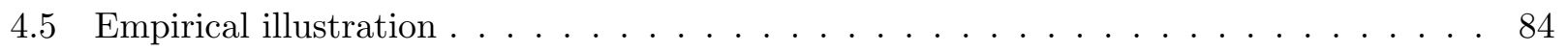

4.5.1 Copula model for exchange rates of Euro-USD and Yen-USD . . . . . . . . 85

4.5.2 Copula model for MSCI indexes of Korea and Singapore . . . . . . . . . . . . 88 


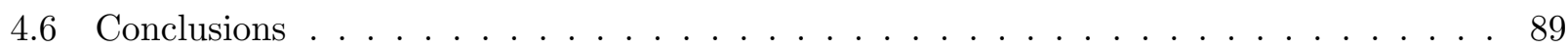

\section{Testing for asset market linkages:}

$\begin{array}{ll}\text { A new approach based on time-varying copulas } & 93\end{array}$

5.1 Introduction . . . . . . . . . . . . . . . . . . . . . . . 93

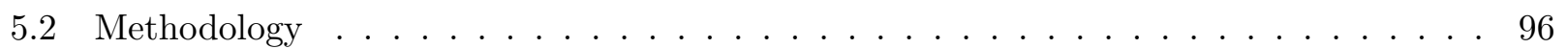

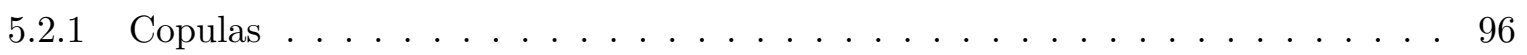

5.2 .2 The model . . . . . . . . . . . . . . . . . . . . 97

5.2.3 Testing for structural breaks in copula models (Dias and Embrechts 2004) . . . 99

5.2.4 Testing for a structural break in unconditional volatility . . . . . . . . . . . . 101

5.2.5 Testing for structural breaks in dependence conditional on a break in volatility: a sequential algorithm . . . . . . . . . . . . . . . . . . 102

5.3 Monte Carlo study . . . . . . . . . . . . . . . . . . . . . . . . . . 105

5.4 Empirical application . . . . . . . . . . . . . . . . . . . . 111

$5.4 .1 \quad$ Data . . . . . . . . . . . . . . . . . . . . 111

5.4 .2 The Sequential Testing Approach $(\mathrm{STA}) \ldots \ldots \ldots \ldots \ldots$. . . . . . . . . . . . . .

5.4 .3 Modeling time-varying dependence . . . . . . . . . . . . . . . . . . 115

5.4 .4 Robustness check . . . . . . . . . . . . . . . . . . . . . . . . . 119

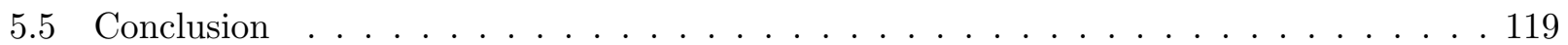

6 Dynamic stochastic copula models: Estimation, inference and applications 121

6.1 Introduction . . . . . . . . . . . . . . . . . . . . . . . . 121

6.2 Specification, estimation and diagnostics . . . . . . . . . . . . . . 123

6.2 .1 The stochastic copula model . . . . . . . . . . . . . . . . . . 123

6.2 .2 Estimation . . . . . . . . . . . . . . . . . . . . . 124

6.2.3 Including the marginals: One Step vs. Two Step Estimation . . . . . . . . . 128

6.2 .4 Estimating the underlying process f . . . . . . . . . . . . 130

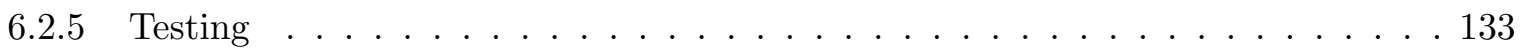

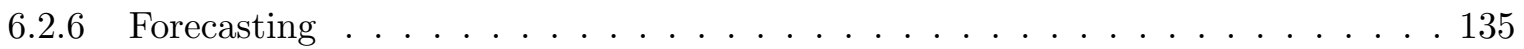

6.2.7 Tail properties of the Gaussian SCAR model . . . . . . . . . . . . . 137

6.2 .8 Possible extensions . . . . . . . . . . . . . . . . . . 137

6.3 Application . . . . . . . . . . . . . . . . . . . . . . . 139

6.3 .1 Daily data: Dow Jones and NASDAQ . . . . . . . . . . . . . . . . 139 
6.3.2 Weekly data: CAC and DAX . . . . . . . . . . . . . . . 146

6.4 Conclusions . . . . . . . . . . . . . . . . . . . . . . . . 149

7 Tails of Gaussian and $t$-copulas with random correlations 151

7.1 Introduction . . . . . . . . . . . . . . . . . . . . . 151

7.2 (Penultimate) Tail dependence coefficients of mixture copulas . . . . . . . . . . . 153

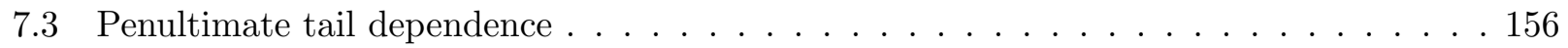

7.3.1 Penultimate tail dependence of mixtures of bivariate $t$-copulas . . . . . . 156

7.3.2 Gaussian copulas with random correlation: Near asymptotic dependence . . . . 157

7.4 Consequences for Modeling . . . . . . . . . . . . . . . . . . . . . . . . 161

7.4 .1 Simulation Study . . . . . . . . . . . . . . . . . . . . . 161

$7.4 .2 \quad$ Empirical Study . . . . . . . . . . . . . . . . . . . . . . 163

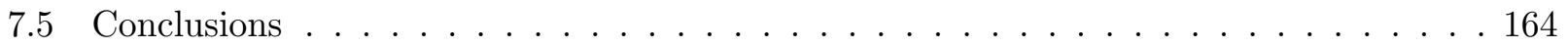

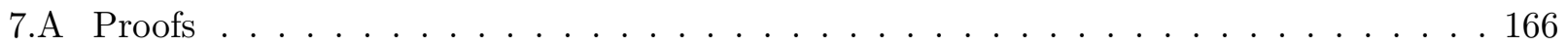

8 Conclusion $r$

8.1 Summary and reflections . . . . . . . . . . . . . . . . . . . . 179

$\begin{array}{ll}\text { Bibliography } & 183\end{array}$

$\begin{array}{ll}\text { Nederlandse samenvatting } & 195\end{array}$

$\begin{array}{ll}\text { Curriculum Vitae } & 199\end{array}$ 


\section{List of Tables}

2.1 Mean Square Errors for different estimators . . . . . . . . . . . . . . . . . 27

2.2 Model selection by AIC when the true model is the Gumbel copula . . . . . . . . . . . 29

2.3 Model selection by AIC when the true model is the Clayton copula . . . . . . . . . . . 30

2.4 Model selection by AIC when the true model is the Frank copula . . . . . . . . . . . 31

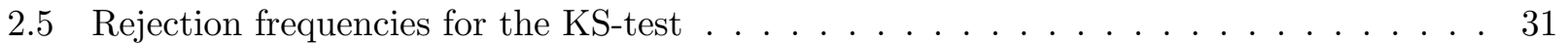

2.6 Rejection frequencies for the $\chi^{2}$ test $\ldots \ldots \ldots \ldots \ldots \ldots \ldots \ldots$

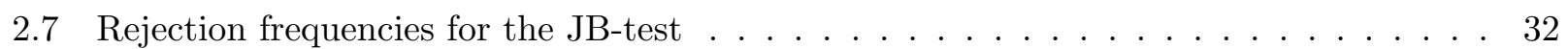

2.8 Goodness-of-fit tests for marginal distributions . . . . . . . . . . . . . . . . 33

2.9 Kendall's tau matrix between the GARCH residuals $\ldots \ldots \ldots$

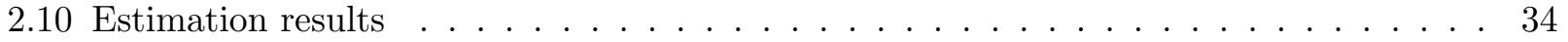

3.1 Rejection frequency of the null of symmetric dependence for data generated by (3.23) 56

3.2 Rejection frequency of the null of symmetric dependence for data generated by (3.31) 58

3.3 Rejection frequency of the null of symmetric dependence for data generated by (3.29) 59

3.4 Application on international stock market returns . . . . . . . . . . . . . . . . 60

3.5 Tests for asymmetric dependence on the autoregressive dynamics of Unemployment

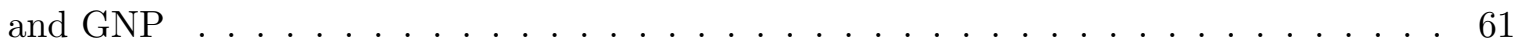

3.6 Tests for asymmetric dependence on the residuals of a linear and nonlinear VAR . . . 61

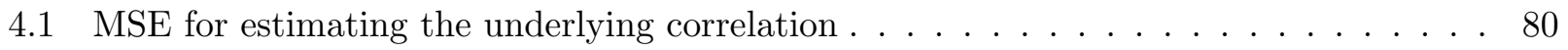

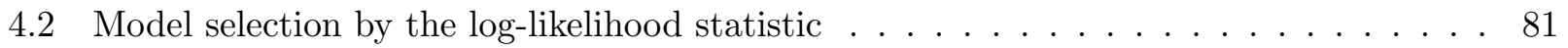

4.3 Size and power of the Anderson-Darling test based on the probability integral transform 82

4.4 First stage estimators: $\mathrm{AR}(\mathrm{p})-\mathrm{GARCH}(1,1)$ model $\ldots \ldots \ldots \ldots$ 
4.5 Model selection for Euro-USD and Yen-USD data . . . . . . . . . . . . . . . . 87

4.6 Model selection for Singapore-Korea MSCI indexes . . . . . . . . . . . . . . . . 90

4.7 Comparison of the presented models . . . . . . . . . . . . . . . . . . 91

5.1 Size of the stability test using DE and STA . . . . . . . . . . . . . 107

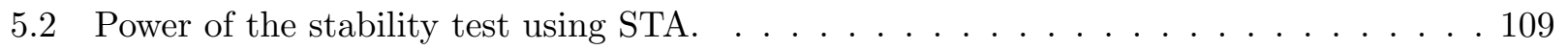

$5.395 \%$ confidence bound for the estimated break date in the dependence parameter breakpoint using STA . . . . . . . . . . . . . . . . . . 110

5.4 Breakpoints in variance. . . . . . . . . . . . . . . . . . . . 113

5.5 Breakpoints in correlation. . . . . . . . . . . . . . . . . . . . . . . 114

5.6 Likelihood ratio tests for significance of variance and correlation break dummies. . . . 116

6.1 Average MSEs for correlation estimates . . . . . . . . . . . . . . . . 134

6.2 Estimates of GARCH and SV models: Dow Jones and Nasdaq . . . . . . . . . . . 140

6.3 Estimates of the stochastic copula model: Dow Jones and Nasdaq. . . . . . . . . . . . 140

6.4 Goodness-of-Fit tests: Dow Jones and Nasdaq. . . . . . . . . . . . . . . . . . . 143

6.5 Out-of-sample fit: Dow Jones and Nasdaq . . . . . . . . . . . . . . . . . . . 145

6.6 Estimation and $\mathrm{GoF}$ for $\mathrm{CAC}$ and DAX returns. . . . . . . . . . . . . . . 147

6.7 Out-of-sample fit: CAC and DAX . . . . . . . . . . . . . . . 149

7.1 Bias estimating $\lambda(u)$ and $\lambda$ using $t$-copulas $\ldots \ldots \ldots \ldots$. . . . . . . . . . . . . .

7.2 Finite level and asymptotic tail dependence of static and dynamic $t$-copulas $\ldots . . .165$ 


\section{List of Figures}

2.1 Illustration of the Gumbel and survival Gumbel copula . . . . . . . . . . . . . . . 16

2.2 Illustration of the Clayton and survival Clayton copula . . . . . . . . . . . . . . 17

2.3 Illustration of the Frank and Plackett copula . . . . . . . . . . . . . . . . 18

3.1 Contour plots and simulated data from the Gumbel and Clayton Copula . . . . . . . 43

$3.2 \tau^{+}(0.5)$ and $\tau^{-}(0.5)$ for the Gumbel and Clayton copulas $\ldots \ldots \ldots \ldots$

3.3 Local power functions against $\sqrt{T}$ alternatives $\ldots \ldots \ldots \ldots$. . . . . . . 48

3.4 Simulated data for example $2 \ldots \ldots \ldots \ldots \ldots \ldots$

3.5 Difference between $\tau^{+}$and $\tau^{-}$for data generated by a SETAR model $\ldots \ldots \ldots 3$

3.6 Power functions of the CM, CM and $J$-test as a function of $\alpha$ in (3.30) $\ldots . . .55$

4.1 Time-varying dependence for exchange rate returns $\ldots \ldots \ldots$. . . . . . . . 86

4.2 Time-varying dependence for stock market returns $\ldots \ldots \ldots$. . . . . . . . . 89

5.1 Returns series . . . . . . . . . . . . . . . . . . . . . 112

5.2 GARCH Variances . . . . . . . . . . . . . . . . . . . . 117

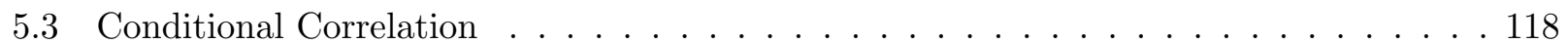

6.1 Volatility estimates: Dow Jones and Nasdaq . . . . . . . . . . . . . . . . . . 141

6.2 Time path of the dependence parameters: Dow Jones and Nasdaq . . . . . . . . . . 142

6.3 Out-of-sample forecasts of the dependence parameter: Dow Jones and Nasdaq . . . . . 144

6.4 Volatility estimates: CAC and DAX . . . . . . . . . . . . . . . . 146

6.5 Time path of the dependence parameters: CAC and DAX . . . . . . . . . . 148

7.1 Penultimate tail dependence of $t$-copulas . . . . . . . . . . . . . . . . 152 
7.2 Tail dependence of static and dynamic t-copulas . . . . . . . . . . . . 155

$7.3 \lambda(u)$ and $\eta$ for the stochastic Gaussian copula . . . . . . . . . . . . . 160 


\section{Introduction}

\subsection{Objective}

The aim of this thesis is to extend existing techniques for measuring and modeling dependencies, and to propose new ones. As this covers quite a large spectrum of potential techniques and applications we will focus on dependence models using so called copula functions. As the realm of applications we are mostly interested in modeling financial time series such as stock (market) returns or exchange rate returns, although many of the techniques dealt with in this thesis may also be applied different types of data. Copula models for financial time series have been studied extensively in both the empirical finance and econometrics literature. In particular the combination with GARCH models for volatility has become a popular way to jointly model multivariate time series. Notable contributions in this field have been made by Jondeau and Rockinger (2006), Lee and Long (2009), Patton (2006b). Also from a theoretical perspective a lot of progress has been made in recent years. Some notable contributions in econometrics are Chen and Fan (2006a,b) who study the properties of a popular semiparametric estimator for time series problems or Patton (2006a) who considers the problem of estimating copula models when the two data series available are of unequal length. At this point we do not want to provide a complete overview over this fast growing field of research, as this has been done elsewhere, for example by Patton (2009) and Embrechts (2009). In fact, Chapter 2 of this thesis has been included exactly for the reason of giving a brief overview on the copula literature and to introduce copulas. Although much work has already been done on the application of copulas in financial econometrics and many issues have been resolved, there are still a large number of open problems. Of course it is not possible to deal with all of them in the context of this thesis and hence important issues such as the development of higher dimensional models have to be ignored. The main issue that will be dealt with is how dependence changes, how these changes can be captured and what this implies for modeling dependence with copulas. Changing dependencies can be seen from different perspectives, two of which are dealt with in this thesis. The first treatment is an unconditional one, 
where the dependence is different for large and for small realizations of the variables of interest. This is what is usually termed asymmetric dependence and is found quite often in financial data when dependence is higher during bear markets than during bull markets. Certain copulas are suited quite well to capture these unconditional asymmetric dependence structures and this is one of the main reasons for the recent interest in copula models for financial data. The second and particularly the third Chapter of this thesis will deal with ways to measure, model and test for such asymmetric dependence structures. The main topic of this thesis, however, will be copulas that vary over time, a feature that appears to be quite important when dealing with time series. Time-varying, or conditional, copulas have been introduced by Patton (2006b). Given that it is a stylized fact that the volatility and correlation of financial time series vary strongly over time the assumption of static dependence is open to doubt. Simple techniques such as measuring dependence over sub samples or rolling windows will in fact almost always suggest that a constant copula model cannot be appropriate. Nevertheless, it is still unclear how to best model time-varying dependence, how to test for it or how to forecast it. This thesis provides answers to such questions and proposes novel ways on how to deal with time-varying copulas. Interestingly not all findings, both theoretical and empirical, correspond to what one would intuitively expect, and these findings were what made the work on this thesis so intriguing. For example, in Chapter 5 it is found that during the Asian crisis the increase in stock market volatility did not coincide with increasing correlations, although this is what is usually claimed in the literature and predicted by theoretical models for stock market returns such as factor models. Another surprising finding was that the tails of Gaussian copulas with stochastic correlations turn out to be quite heavy and that consequently time-varying Gaussian copulas become very attractive for modeling the distribution of many financial time series.

\subsection{Structure of the thesis}

Chapter 2 of this thesis serves as an introduction to copula functions and to techniques on how to handle them. An understanding of some of the concepts introduced here should greatly help the reader to be able to follow the rest of the thesis, although the chapters are mostly self contained. Formal definitions of copulas and related concepts are provided and the most important theoretical results are reviewed. Commonly used copula based measures of dependence are also introduced, before providing a catalogue of the most popular parametric copulas along with a brief discussion of their properties and methods to simulate data from them. Next, different ways to estimate the parameters of copulas are explained and the important issue of model selection is shortly discussed. 
A Monte Carlo study then compares the competing estimators for the copula parameter and the suggested methods for evaluating the goodness-of-fit. Finally, different copula models are estimated for a number of bivariate exchange rate series of Latin American currencies against the US dollar and the best fitting ones are identified.

Chapter 3 deals entirely with asymmetric dependence, so situations where dependence is different for large and small realizations of a variable of interest. Although this issue is also briefly touched in Chapter 2 by locking at the coefficient of tail dependence in the upper and lower tail, Chapter 3 defines asymmetric dependence differently by locking at asymmetries in the whole distribution. To this end a conditional version of Kendall's tau, a popular rank correlation coefficient, is defined and formulas are provided to evaluate it for any copula of interest. Two likelihood ratio tests based on well known copulas are proposed to test the null hypothesis of symmetric dependence. Our test statistics have non-standard asymptotic distributions, but these have been dealt with in the literature by Vuong (1989) and Chen and Fan (2006a). The tests are shown to have better size properties and higher power than a competing test proposed by Hong, Tu, and Zhou (2007). Additionally, we suggest three examples of data generating processes that can lead to asymmetric dependence and study these both analytically and in a Monte Carlo framework. Finally, we illustrate the use of our tests on stock market returns and on quarterly US GNP and Unemployment data and we find evidence of asymmetries and nonlinearities.

Chapter 4 then provides the transition to the topic of time-varying copulas by reviewing the literature on that relatively new topic and by systematically comparing different models. In particular, we review eight competing specifications for time-varying copulas and we briefly discuss how they are estimated. We then discuss how the goodness-of-fit can be compared and tested for in the case of time-varying copulas. A simulation study compares the ability of the competing techniques to estimate the underlying time-varying dependence parameter under quite different assumptions on how it is generated. Also the goodness-of-fit criteria are analyzed in this Monte Carlo study. An empirical application provides an illustration of the different methods for exchange rate and stock market return data measured at different frequencies. Apart from evaluating the statistical fit of the different models we also compare their performance by means of the Value-at-Risk.

Chapter 5 deals with testing for financial contagion during the Asian crisis relying on copula based techniques. A new approach to test for increases in stock market interdependence after a financial crisis, also labeled shift contagion, is proposed here. To this end the structural break test for copulas introduced by Dias and Embrechts (2004) is extended to allow for breakpoints in unconditional 
volatility at possibly distinct points in time. A sequential algorithm is proposed to reduce the computational burden of finding multiple unknown breakpoints and a parametric bootstrap is used to obtain appropriate critical values for our testing procedure. Applied to the recent 1997 Asian crisis, the analysis confirms that breaks in variances always precede those in the dependence parameter. Moreover, a significant "J-shape" evolution of the dependence parameter is detected supporting the idea of shift-contagion.

In Chapter 6 a new time-varying copula model, the stochastic copula autoregressive (SCAR) model, is introduced and various aspects of this model are studied. This model was motivated by stochastic volatility models by having time-varying dependence parameters that are driven by a latent Gaussian AR(1) process. The problem of estimating this model is overcome by adapting the efficient importance sampling technique by Richard and Zhang (2007) to the case at hand. We discuss how to estimate the latent dependence process, how to perform specification tests for this models class, and how to forecast the copula parameter. In both a Monte Carlo simulation and two empirical applications looking at in-sample and out-of-sample fit this model is compared to the DCC GARCH model of Engle (2002) and the dynamic copula model of Patton (2006b).

Chapter 7 studies the tail behavior of Gaussian and $t$-copulas when the correlation parameter is driven itself by a latent random process. This was motivated by the SCAR model introduced in Chapter 6 , but covers more general cases as well. We find that both penultimate and asymptotic tail dependence are much larger for elliptical copulas with random correlations than for static ones with the same unconditional correlation. Furthermore, we show for Gaussian and Student copulas that the penultimate coefficient of tail dependence is generally larger than its limit, the coefficient of tail dependence, which can have serious consequences for estimation and evaluation of extreme risk. Finally, for Gaussian copulas when mixing over the correlation parameter $\rho$, one obtains copulas whose tails inherit the property of asymptotic independence, but which at the same time fall into a new class of asymptotic dependence, which we label near asymptotic dependence, meaning that at practically relevant quantiles the tails are dependent. We provide several numerical illustrations of our theoretical findings and discuss the consequences for modeling by performing a simulation study and an illustration using financial time series.

Finally, Chapter 8 summarizes the most important results and provides a short discussion of current and future issues in the related literature. 


\section{2}

\section{Estimation and model selection of copulas with an} application to exchange rates ${ }^{1}$

\subsection{Introduction}

An assumption that is often made about the (joint) distribution of financial variables is that of normality. The dependence between variables that have a multivariate normal distribution is purely determined by the linear correlation coefficient. However, empirical findings show that asset returns have skewed and leptokurtic marginal distributions and that the dependence between these asset returns goes beyond the simple linear form. There is evidence that extreme co-movements (known as tail area dependence) occur and that some markets may be more dependent during extreme downward movements then when they are moving upwards. Simply looking at linear correlation in a non-elliptical world can be misleading as described by Embrechts, McNeil, and Straumann (2002). Copula functions allow for modeling joint multivariate distributions in a simple and extremely flexible way. Copulas are able to yield any kind of dependence structure independently of the marginal distributions. Whereas the bivariate Normal distribution requires its margins to be normally distributed as well, a Gaussian copula is characterized by the correlation coefficient if the margins were normally distributed. They can take on any distribution, which need not even be equal for all the margins. This explains the simple algorithm for simulating data from a Gaussian copula, which simply requires imposing linear dependence on a number of normally distributed variables by pre-multiplying the series with the Cholesky decomposition of the desired covariance matrix and then using Fisher's probability integral transform to give the marginals any distribution desired. Other copulas cannot be understood in such a simple way and they allow for very different types of nonlinear dependence. This may be depicted by graphing the relationship between the parameter of a given copula and the corresponding linear correlation coefficient, which will be a nonlinear one.

\footnotetext{
${ }^{1}$ This chapter is based on Manner (2007).
} 
The Latin word "copula" means "link, tie, bond". Copulas (or copulae when using the Latin plural) were first introduced by Sklar (1959), who proved the main result on copulas known as Sklar's theorem. They offer scale invariant measures of dependence, so dependence is not affected by increasing transformations in any of the variables. Their use in econometrics developed over the last 15 years, but they become more and more popular as more useful applications in finance arise. One application where copulas turn out to be very useful is quite obviously the Value-at-Risk of a portfolio, as it might differ quite significantly when comparing its value under the assumption of joint normality and when having heavy tailed margins and a copula that allows for a higher dependence during market downturns, see for example Junker and May (2005). Copulas offer further applications in risk management like modeling joint defaults for credit risks or when pricing exotic options with two or more underlying assets. Cherubini, Luciano, and Vecchiato (2004) show many applications of copulas in finance. Further uses are the construction of investment portfolios and the more realistic simulation of asset returns. Another application suggested is modeling autoregressive dynamic processes using copulas to capture the time dependence in one variable rather than the dependence between two or more variables as in Bouyé, Gaussel, and Salmon (2001) or Chen and Fan (2006b). More mathematical treatments of copulas are the books by Nelsen (2006) and Joe (1997) or the review by Embrechts, Lindskog, and McNeil (2003).

In this chapter we aim at reviewing the theory needed to understand copula based modeling and apply it to a given data set. We focus mainly on techniques for simulating random observations from copulas, the different ways of estimating copulas and some simple model selection techniques. We contribute to the issue of model selection by showing that selecting the candidate copula that produces the highest Akaike information criterion is a very reliable method and that using the JarqueBera test on the appropriately transformed data performs better than some alternative tests that have been suggested in the literature.

The chapter is structured as follows. The underlying theory is developed in Section 2.2, including some commonly used measures of dependence and the algorithms used to simulate data from a given copula. Furthermore we present the mostly used copula functions and their properties. The estimation and testing of a given copula model will be discussed in Section 2.3 and the performance of the different methods will be analyzed with the help of Monte Carlo studies in Section 2.4. In Section 2.5 their practical use will be illustrated by modeling the joint distribution of exchange rates of Latin American currencies against the Euro. Finally, we conclude in Section 2.6. 


\subsection{Introducing copulas and related concepts}

A copula can be seen as a correspondence, which assigns the value of the joint distribution function to each ordered pair of values of the individual distribution functions. Alternatively it can be seen as the joint distribution function of a set of uniformly $(0,1)$ distributed random variables or simply as a function, which couples, or joins, the marginal distributions with their multivariate distribution function. These "operational" definitions serve as a good intuition about what a copula function is.

All results in this section are derived for the bivariate case. For an extension to the multivariate case, which is only trivial for very few cases, see Nelsen (2006). Also proofs for most of the results mentioned can be found there.

\subsubsection{Preliminaries and copulas defined}

Before we are able to introduce copula functions themselves a number of properties need to be presented. First of all the notion of a nondecreasing function has to be generalized for the multivariate setting. We begin by defining the concept of a 2 -increasing function. Note that $\overline{\mathbf{R}}$ denotes the extended real line.

Definition 2.1. Let $\mathbf{S}_{\mathbf{1}}$ and $\mathbf{S}_{\mathbf{2}}$ be nonempty subsets of $\overline{\mathbf{R}}$, and let $H$ be a function such that DomH $=$ $\mathbf{S}_{\mathbf{1}} \times \mathbf{S}_{\mathbf{2}}$. Let $B=\left[x_{1}, x_{2}\right] \times\left[y_{1}, y_{2}\right]$ be a rectangle all of whose vertices are in Dom $H$. Then the $\mathrm{H}-$ volume of $B$ is given by:

$$
V_{H}(B)=H\left(x_{2}, y_{2}\right)-H\left(x_{2}, y_{1}\right)-H\left(x_{1}, y_{2}\right)+H\left(x_{1}, y_{1}\right)
$$

A natural interpretation of the $\mathrm{H}-$ Volume is when $\mathrm{H}$ is a distribution function. It then represents the probability of an event occurring in the region specified, which is a rectangle in the 2-dimensional case.

Definition 2.2. A 2-place real function $H$ is 2-increasing if $V_{H}(B) \geqslant 0$ for all rectangles $B$ whose vertices lie in DomH.

We call a function from $\mathbf{S}_{\mathbf{1}} \times \mathbf{S}_{\mathbf{2}}$ into $\mathbf{R}$ grounded if there is a least element in $S_{1}$, as well as in $S_{2}$, let's say $a_{1}$ and $a_{2}$, such that $\mathrm{H}\left(\mathrm{x}, a_{2}\right)=0$ and $\mathrm{H}\left(a_{1}, \mathrm{y}\right)=0$ for all $(x, y)$ in $\mathbf{S}_{\mathbf{1}} \times \mathbf{S}_{\mathbf{2}}$. A function that is grounded and 2-increasing is known to be increasing in each argument. Another property that will be useful later on is the following. Let $\mathbf{S}_{\mathbf{1}}$ and $\mathbf{S}_{\mathbf{2}}$ have a greatest element, $b_{1}$ and $b_{2}$ respectively. 
Then the one dimensional marginals of $H(x, y)$ are given by $F(x)=H\left(x, b_{2}\right)$ and $G(y)=H\left(b_{1}, y\right)$. With this at hand we are ready to present the definition of a copula.

Definition 2.3. A two dimensional copula is a function $C$ from $\mathbf{I}^{2}$ to $\boldsymbol{I}$ such that

1. $C$ is grounded and 2-increasing.

2. $C(u, 1)=u$ and $C(1, v)=v$ (margins)

Hence a copula is no more than a function with the unit cube $\mathbf{I}^{2}$ as its domain, the unit interval $\mathbf{I}$ as its range, and that is increasing in each element and has marginals.

The main result about copula functions is Sklar's theorem (Sklar 1959), which shows why copulas are so useful when dealing with multivariate distribution functions.

Theorem 2.1 (Sklar's theorem for continuous distributions). Let $F$ be the distribution of $X, G$ be the distribution of $Y$, and $H$ be the joint distribution of $(X, Y)$. Assume that $F$ and $G$ are continuous. Then there exists a unique copula $C$ such that

$$
H(x, y)=C(F(x), G(y)), \forall(x, y) \in \overline{\mathbf{R}} \times \overline{\mathbf{R}}
$$

Conversely, if we let $F$ and $G$ be distribution functions and $C$ be a copula, then the function $H$ defined by equation (2.2) is a bivariate distribution function with marginal distributions $F$ and $G$.

In other words, the joint distribution can be represented separately by the marginal distribution functions and the copula, which completely describes the dependence between the i.i.d. random variables $X$ and $Y{ }^{2}$ The converse turns out to be very useful in the construction of multivariate distribution functions, as we now can take any pair of marginal distributions and any copula to construct a bivariate distribution. This allows for a large number of multivariate distribution functions that can be constructed easily.

There is a very useful corollary to Sklar's theorem, which allows us to represent the copula by the joint distribution function and the inverses of the marginals. To ensure the existence of these inverses a new concept of the inverse of a function is required, which is called the quasi-inverse.

Definition 2.4. The quasi-inverse, $F^{(-1)}$ of a distribution function $F$ is defined as

$$
F^{(-1)}(u)=\inf \{x: F(x) \geqslant u\} \text { for } u \in[0,1]
$$

\footnotetext{
${ }^{2}$ The general discussion of the theory will be in an i.i.d. setting and we denote random variables by capital letters, realizations by lower case letters and we add an index $t$ when we speak of a sample.
} 
Corollary 2.1. Let $H$ be any bivariate distribution with continuous marginal distributions $F$ and $G$. Let $F^{(-1)}$ and $G^{(-1)}$ denote the (quasi-) inverses of the marginal distributions. Then there exists a unique copula $C$ from $\mathbf{I}^{2}$ to $\mathbf{I}$ such that

$$
C(u, v)=H\left(F^{(-1)}(u), G^{(-1)}(v)\right), \forall(u, v) \in \mathbf{I}^{2}
$$

The next result answers the natural question, whether there is an upper and a lower bound that holds for every copula.

Theorem 2.2 (Frèchet-Hoeffding bounds inequality). Let $C$ be a copula. The for every $(u, v)$ in $\mathbf{I}^{\mathbf{2}}$

$$
W(u, v)=\max (u+v-1,0) \leqslant C(u, v) \leqslant \min (u, v)=M(u, v)
$$

The upper bound corresponds to perfect positive dependence between two variables, the lower bound to perfect negative dependence. Additionally consider the function $\Pi(u, v)=u v$, which, not surprisingly, corresponds to independence. In the bivariate case these three important functions are copulas. For $n \geqslant 3$, however, the function $W$ is not a copula.

Finally, consider the function

$$
\hat{C}(u, v)=u+v-1+C(1-u, 1-v),
$$

which is the copula of a joint survival function. This is known as the survival or rotated copula.

As copulas are used to model dependencies one must specify how to measure the strength of overall dependence. Traditionally the dependence between two random variables is measured by the linear correlation coefficient. However, when the dependence is not described by an elliptical distribution it can be quite misleading to use linear correlation and it might be more reasonable to use copula based measures of dependence, which are scale invariant (see Embrechts et al. (2002) for caveats on using the correlation coefficient for measuring dependence). One of these more robust copula based measures is Kendall's tau. It has become the most popular measure of overall dependence in the literature on copulas and it relies on the concept of concordance. Consider two pairs of observations $\left(x_{i}, y_{i}\right)$ and $\left(x_{j}, y_{j}\right)$ from the continuous random variables $(X, Y)$. We call these pairs of observations concordant if $\left(x_{i}-x_{j}\right)\left(y_{i}-y_{j}\right)>0$ and discordant if $\left(x_{i}-x_{j}\right)\left(y_{i}-y_{j}\right)<0$. Hence, two random variables are said to be concordant, when large values of one random variable are associated with large values of the other, and similarly small values tend to be associated with each other. 
Using the concept of concordance we are now able to introduce the association measure Kendall's tau. Its sample version is defined as the fraction of concordant pairs of observations in the sample minus the fraction of discordant pairs of observations. The population version of Kendall's tau is defined as the difference between the probability of concordance and the probability of discordance.

$$
\tau=\tau_{X, Y}=P\left[\left(X_{1}-X_{2}\right)\left(Y_{1}-Y_{2}\right)>0\right]-P\left[\left(X_{1}-X_{2}\right)\left(Y_{1}-Y_{2}\right)<0\right]
$$

Kendall's tau may be represented as a function of the expected value of a copula as follows.

$$
\tau_{C}=4 E(C(U, V))-1
$$

A proof of this relation can be found in Chapter 3. For some copulas there is a one to one relationship between its parameter and Kendall's tau.

Another frequently encountered and important dependence concept, which is relevant when modeling extreme events, is tail dependence. It measures the dependence of the random variables $X$ and $Y$ in the upper-right-quadrant and lower-left-quadrant. As the measure discussed above it is a copula property and hence it is invariant under strictly increasing transformations of the random variables. There are two alternative definition for the coefficient of upper tail dependence. We first state the probabilistic one, followed by the definition in terms of copulas.

Definition 2.5. Let $(X, Y)$ be a vector of continuous random variables with marginal distribution functions $F$ and $G$. The coefficient of upper tail dependence of $(X, Y)$ is

$$
\lambda_{U}=\lim _{u \uparrow 1} P\left[Y>G^{-1}(u) \mid X>F^{-1}(u)\right]
$$

Definition 2.6. If a bivariate copula $C$ is such that

$$
\lambda_{U}=\lim _{u \uparrow 1} \frac{1-2 u+C(u, u)}{1-u}
$$

exists, then $C$ has upper tail dependence if $\lambda_{U} \in(0,1]$, and upper tail independence if $\lambda_{U}=0$.

In a similar way the concept of lower tail dependence can be introduced. Only the definition corresponding to definition 2.6 is considered here. 
Definition 2.7. If a bivariate copula $C$ is such that

$$
\lambda_{L}=\lim _{u \downarrow 0} \frac{C(u, u)}{u}
$$

exists, then $C$ has lower tail dependence if $\lambda_{L} \in(0,1]$, and lower tail independence if $\lambda_{L}=0$.

Alternative formulas for $\lambda_{U}$ and $\lambda_{L}$ exist and can be found in Embrechts et al. (2003). There it is shown that the coefficient of upper tail dependence for a copula is equal to the coefficient of lower tail dependence for the corresponding survival copula and the other way around. We will encounter some illustrations of tail dependence in the next section, where some families of copulas are introduced. In Chapter 7 we look more closely at the coefficient of tail dependence and related measures of dependence in the extremes of the distribution.

\subsubsection{Examples and families of copulas}

In this section the most commonly used copulas will be described and their properties will be presented. The presentation is far from complete, but covers the copulas that are considered in most applications in the literature. For exhaustive lists of copula functions and various methods for constructing copulas the books by Joe (1997) and Nelsen (2006) may be consulted.

\section{Elliptical copulas}

Elliptical copulas are simply the copulas of elliptical distributions. They share a number of properties of the multivariate normal distribution and they are used to model multivariate extreme events and non-normal dependencies. As a result of the fact that simulations from multivariate elliptical distributions are easy to perform, simulations from elliptical copulas are easy to perform as well. An advantage of using elliptical copulas is that we are now able to model multivariate distributions where the marginals are not assumed to be equal (or even of the same family of distributions), but the dependence between the marginals is still characterized by an elliptical distribution (of the uniform marginals). A drawback is that the distribution functions do not have a closed form expression and that elliptical copulas are restricted to have radial symmetry, i.e. $C=\hat{C}$.

The first copula presented is the (bivariate) Gaussian copula. It can easily be derived from the bivariate normal distribution and has the following distribution function

$$
C_{\text {Gaussian }}(u, v)=\int_{-\infty}^{\phi^{-1}(u)} \int_{-\infty}^{\phi^{-1}(v)} \frac{1}{2 \pi \sqrt{1-\rho^{2}}} \exp \left(-\frac{s^{2}-2 \rho s t+t^{2}}{2\left(1-\rho^{2}\right)}\right) d s d t
$$


where $\rho$ is the linear correlation coefficient of the corresponding bivariate normal distribution. Note that it can be shown that the Gaussian copula does not have tail dependence for $\rho<1$. The expression for Kendall's tau is given by

$$
\tau=\frac{2}{\pi} \arcsin (\rho)
$$

Conversely, a non-parametric estimator of $\rho$ is $\sin \left(\frac{\pi \hat{\tau}}{2}\right)$, which is easy to compute and inherits the robustness properties of Kendall's tau.

An elliptical copula that exhibits upper and lower tail dependence is the t-copula given by

$$
C_{t}(u, v)=\int_{-\infty}^{t^{-1}(u ; \nu)} \int_{-\infty}^{t^{-1}(v ; \nu)} \frac{1}{2 \pi \sqrt{1-\rho^{2}}}\left(1+\frac{s^{2}+t^{2}-2 \rho s t}{\nu\left(1-\rho^{2}\right)}\right)^{-\frac{\nu+2}{2}} d s d t
$$

Again, $\rho$ denotes the linear correlation coefficient of the corresponding bivariate t-distribution with $\nu$ degrees of freedom. The relationship between Kendall's tau and $\rho$ is the same as for the Gaussian copula. The coefficients of upper and lower tail dependence, which are equal, are given by

$$
\lambda=2 \bar{t}_{\nu+1}\left(\frac{\sqrt{\nu+1} \sqrt{1-\rho}}{\sqrt{1+\rho}}\right) .
$$

Consequently, $\lambda$ is increasing in $\rho$ and decreasing in $\nu$.

\section{Archimedean copulas}

Archimedean copulas form a large family of copulas with a number of convenient properties and they allow for a large number of dependence structures. Most have closed form expressions, which turns out to be very useful for estimation. They are, unlike many other copulas, not constructed from multivariate distributions using Sklar's theorem. Let $\varphi$ denote the so called generator function of a copula with the following properties:

1. $\varphi(1)=0$

2. $\varphi^{\prime}(t)<0 \forall \mathrm{t} \in(0,1)$ (i.e. it is decreasing)

3. $\varphi^{\prime \prime}(t) \geqslant 0 \forall t \in(0,1)$ (i.e. it is convex)

Now let $\varphi^{[-1]}$ denote the pseudo-inverse, which is equal to the normal inverse for $t \in[0, \varphi(0)]$ and is 
equal to 0 for $t \geqslant \varphi(0)$. Then the Archimedean copula corresponding to the generator $\varphi$ is given by

$$
C(u, v)=\varphi^{[-1]}(\varphi(u)+\varphi(v))
$$

When $\varphi(0)=\infty$ we say that $\mathrm{C}$ is strict and the pseudo inverse is simply the standard inverse of a function. Archimedean copulas are symmetric, i.e. $\mathrm{C}(\mathrm{u}, \mathrm{v})=\mathrm{C}(\mathrm{v}, \mathrm{u})$, and they are associative, i.e. $\mathrm{C}(\mathrm{C}(\mathrm{u}, \mathrm{v}), \mathrm{w})=\mathrm{C}(\mathrm{u}, \mathrm{C}(\mathrm{v}, \mathrm{w}))$. A very convenient property is that Kendall's tau and the coefficients of tail dependence can be expressed in terms of the generator functions. These expressions are:

$$
\begin{gathered}
\tau_{C}=1+4 \int_{0}^{1} \frac{\varphi(t)}{\varphi^{\prime}(t)} d t \\
\lambda_{U}=2-2 \lim _{s \downarrow 0} \frac{\varphi^{-1^{\prime}}(2 s)}{\varphi^{-1^{\prime}}(s)} \\
\lambda_{L}=2 \lim _{s \rightarrow \infty} \frac{\varphi^{-1^{\prime}}(2 s)}{\varphi^{-1^{\prime}}(s)} .
\end{gathered}
$$

In the following some examples of Archimedean copulas will be given.

For $\varphi(t)=-\ln (t)$ we obtain the product copula $\Pi$. Also the Frèchet-Hoeffding bounds are limiting cases of Archimedean copulas. The most commonly used cases are:

\section{Clayton copula:}

$\varphi(t)=\frac{t^{-\theta}-1}{\theta}, \theta \in[-1, \infty] \backslash\{0\}$

Its distribution function is:

$$
C_{\theta}^{\text {Clayton }}(u, v)=\max \left[\left(u^{-\theta}+v^{-\theta}-1\right)^{\frac{-1}{\theta}}, 0\right]
$$

For $\theta>0$ the Clayton copula is strict and has lower tail dependence. The coefficient of lower tail dependence is given by $\lambda_{L}=2^{-1 / \theta}$. The expression of Kendall's tau can be shown to be $\tau=\frac{\theta}{\theta+2}$. Furthermore $C_{-1}=W, \lim _{\theta \rightarrow 0} C_{\theta}=\Pi$ and $\lim _{\theta \rightarrow \infty} C_{\theta}=M$

\section{Gumbel copula:}

$\varphi(t)=(-\ln (t))^{\theta}$, where $\theta \geqslant 1$

$$
C_{\theta}^{\text {Gumbel }}(u, v)=\exp \left(-\left[(-\ln (u))^{\theta}+(-\ln (v))^{\theta}\right]^{1 / \theta}\right)
$$

For Kendall's tau the relation $\tau=1-\frac{1}{\theta}$ holds and the Gumbel copula has upper tail dependence 
$\lambda_{U}=2-2^{1 / \theta}$, but no lower tail dependence.

Similarly to the case above $C_{1}=\Pi$ and $\lim _{\theta \rightarrow \infty} C_{\theta}=M$.

\section{Frank copula:}

$\varphi(t)=-\ln \left(\frac{e^{-\theta t}-1}{e^{-\theta}-1}\right)$, where $\theta \neq 1$

$$
C_{\theta}^{F r a n k}(u, v)=-\frac{1}{\theta} \ln \left(1+\frac{\left(e^{-\theta u}-1\right)\left(e^{-\theta v}-1\right)}{e^{-\theta}-1}\right)
$$

It holds that $\tau=1-\frac{4\left(1-D_{1}(\theta)\right.}{\theta}$, where $\mathrm{D}$ is the Debye function

$$
D_{k}(x)=\frac{k}{x^{k}} \int_{0}^{x} \frac{t^{k}}{e^{t}-1} d t
$$

Frank copulas display the property of radial symmetry and do not have any tail dependence. In fact the Frank copula was shown to be the only Archimedean copulas that has radial symmetry.

\section{Joe-Clayton copula:}

The distribution function of the Joe-Clayton copula is

$$
C_{\lambda_{U}, \lambda_{L}}^{J C}=1-\left(1-\left[\left[1-(1-u)^{\kappa}\right]^{-\gamma}+\left[1-(1-v)^{\kappa}\right]^{-\gamma}-1\right]^{-1 / \gamma}\right)^{1 / \kappa}
$$

where

$$
\kappa=\frac{1}{\log _{2}\left(2-\lambda_{U}\right)} \text { for } \lambda_{U} \in(0,1)
$$

and

$$
\gamma=\frac{-1}{\log _{2}\left(\lambda_{L}\right)} \text { for } \lambda_{L} \in(0,1) .
$$

When $\lambda_{U}=0$ it collapses to the Clayton copula. As one of the coefficients approaches 1 the JoeClayton copula approaches the Frèchet-Hoeffding upper bound. The two parameters $\lambda_{U}$ and $\lambda_{L}$ measure the coefficient of upper and lower tail dependence respectively. Equality of the two parameters does not imply symmetry. Patton (2006b) introduced a symmetrized version of the Joe-Clayton copula, which has this desirable property.

\section{BB1 copula:}

$C_{\theta, \delta}^{B B 1}=\left(1+\left(\left(u^{-\theta}-1\right)^{\delta}+\left(v^{-\theta}-1\right)^{\delta}\right)^{1 / \delta}\right)^{-1 / \theta}$ 
When $\delta$ is equal to 1 it becomes the Clayton copula. As $\theta \rightarrow 0$ it becomes the Gumbel copula. Furthermore, $\lambda_{U}=2-2^{1 / \delta}$ and $\lambda_{L}=2^{-1 / \delta \theta}$.

\section{Further copulas}

Although there are many copulas that are neither elliptical nor Archimedean we just present the ones that are useful in the context of this thesis.

\section{Plackett copula:}

$$
C_{\theta}^{\text {Plackett }}(u, v)=\frac{1}{2}(\theta-1)\left[1+(\theta-1)(u+v)-\sqrt{\left[(1+(\theta-1)(u+v))^{2}-4 \theta(\theta-1) u v\right]}\right]
$$

Additionally, $\theta \in(0, \infty)$ and $\lim _{\theta \rightarrow 1} C_{\theta}=\Pi$. The Plackett copula has upper tail dependence as $\theta$ goes to infinity and lower tail dependence as $\theta$ goes to 0 .

\section{Rotated copulas:}

The idea of rotating a copula function makes sense only for ones with an asymmetric dependence structure. In this chapter we will make use of the rotated Gumbel and the rotated Clayton copulas. In practical terms, if $u$ and $v$ have e.g. a Gumbel copula, then the variables $1-u$ and $1-v$ have the rotated Gumbel copula, which instead of upper tail dependence shows stronger dependency in the lower tail. Rotated copulas are also called survival copulas of the corresponding family. Note that the survival copula of an Archimedean copula is not Archimedean.

Next to the copula families introduced, one can consider a mixture of two or more copulas, which is simply the convex combination between the copula functions considered. This makes it possible to obtain any dependence structure desired.

In Figures 2.1-2.3 we present scatterplots and contour lines for some of the most popular copulas with standard normal margin and parameters corresponding to Kendall's tau equal to 0.5. The difference in the dependence structure and the asymmetries for the Clayton and Gumbel copulas are quite easy to see.

\subsubsection{Simulation from a copula}

A possible application of copula functions is the efficient simulation of an asset return distribution in a more realistic way or, more generally, simulation from any distribution with dependent observa- 
Figure 2.1: Illustration of the Gumbel and survival Gumbel copula

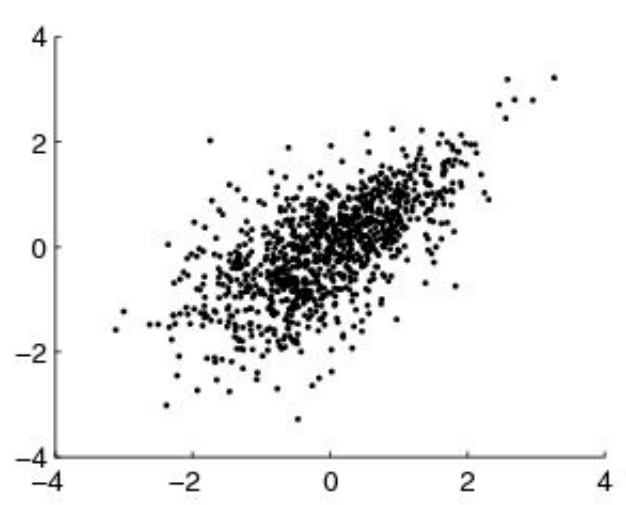
Gumbel $(\mathrm{x}, \mathrm{y}, 2)$
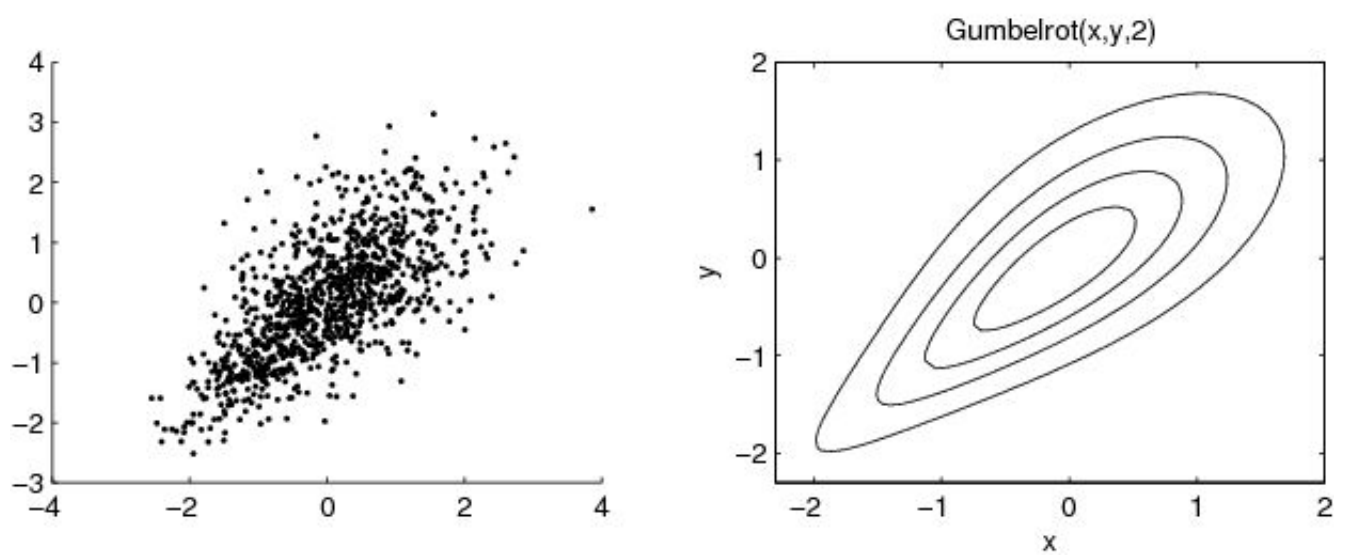

tions. By using the right copula, or even a mixture of two or more copulas, and the corresponding parameters any dependence structure may be imposed. The simulated data offers itself to a variety of Monte Carlo methods.

\section{Conditional sampling}

The following algorithm is the most general one that can be used to simulate a sample from any copula. However, the method is not always the most efficient one, so more efficient ways will be discussed below. Whenever one of the algorithms described below is applicable it should be used. The marginal series obtained by the algorithm have the $U(0,1)$ distribution, but as a result of Sklar's theorem can be transformed into any distribution without changing the dependence structure using the method above. The following algorithm is known as the conditional distribution method. The conditional distribution can be obtained as follows.

$$
C(v \mid u)=P[V \leqslant v \mid U=u]=\frac{\partial C(u, v)}{\partial u}
$$


Figure 2.2: Illustration of the Clayton and survival Clayton copula
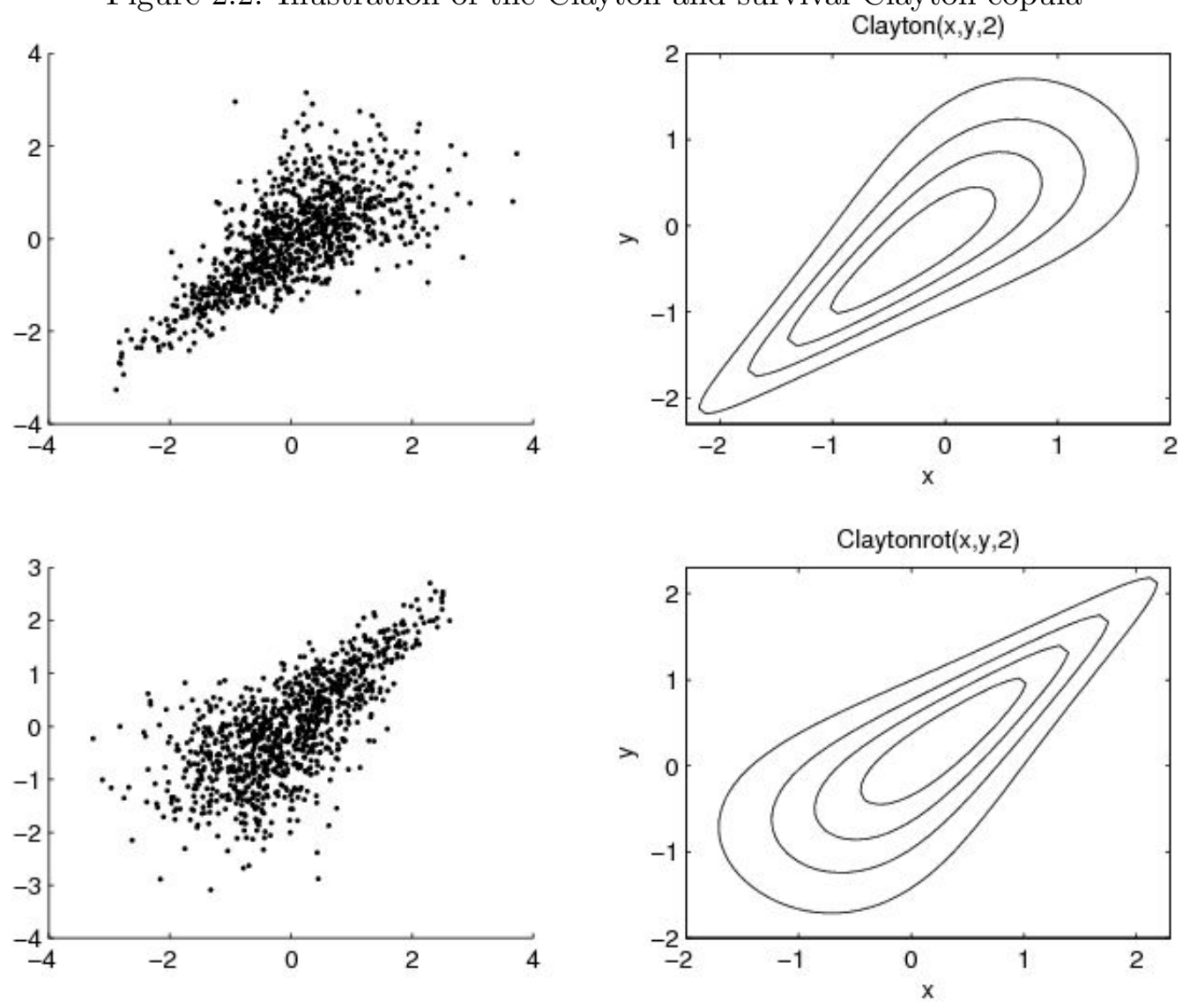

1. Generate two independent uniform $(0,1)$ variates $\mathrm{u}$ and $\mathrm{t}$;

2. Set $\mathrm{v}=C^{(-1)}(t \mid u)$, where $C^{(-1)}$ denotes the quasi-inverse of $C(v \mid u)$.

3. The pair $(u, v)$ has joint distribution function $C$.

Note that if the inverse of $C$ cannot be found analytically, it has to be obtained using numerical root finding. Unfortunately, this makes the algorithm particularly slow.

\section{Simulation from elliptical copulas}

Let $\Omega$ denote the covariance matrix, which is positive definite, and let $A$ be defined such that $\Omega=A A^{T}$. Then if $\mathbf{Z}=Z_{1}, \ldots, Z_{m} \stackrel{i . i . d .}{\sim} N(0,1)$ it follows that

$$
\mu+A \mathbf{Z} \sim N_{m}(\mu, \Omega)
$$

This can be used to efficiently simulate random variates from a Gaussian copula using the Cholesky 
Figure 2.3: Illustration of the Frank and Plackett copula
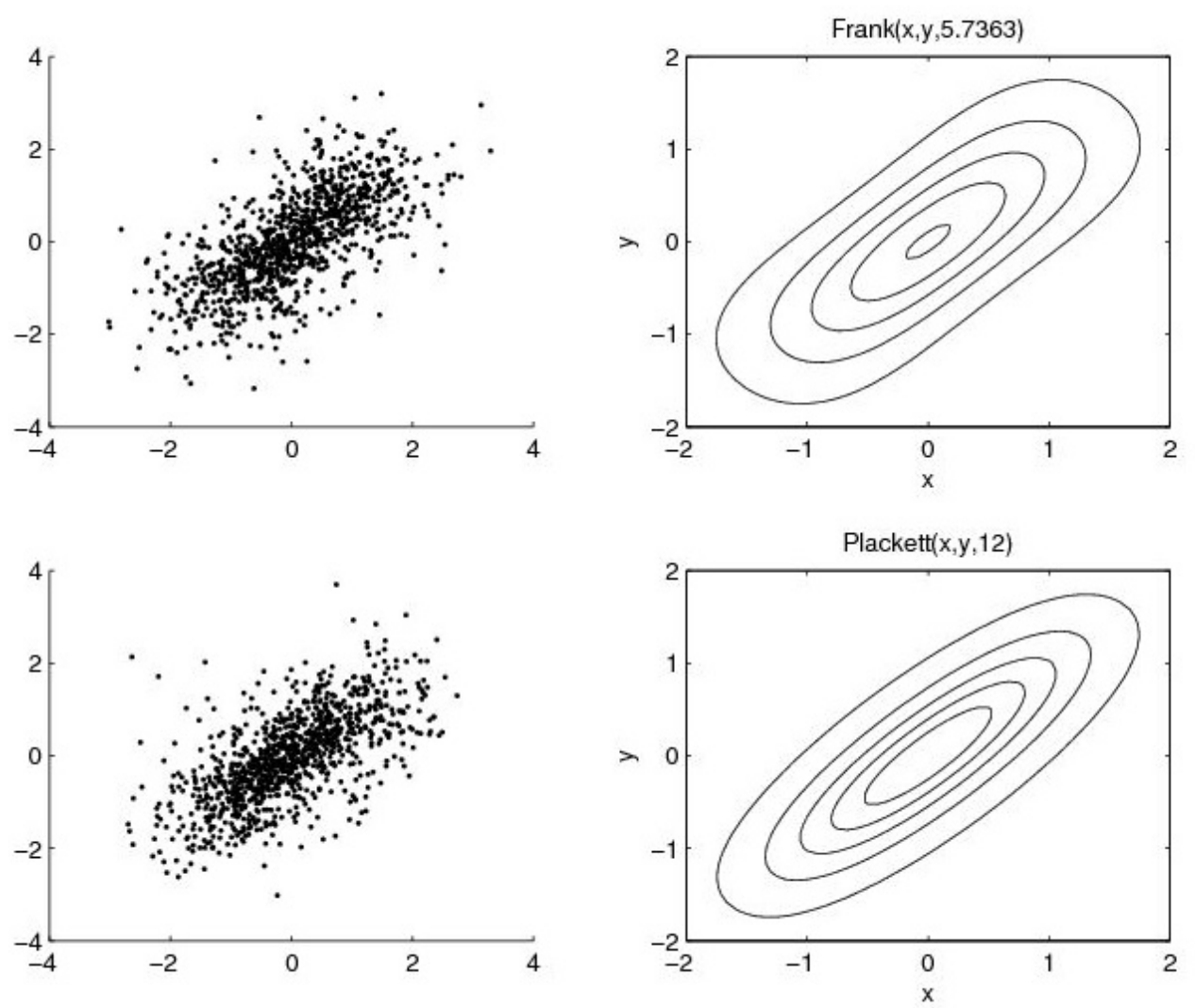

decomposition to impose the dependence on the independent random variables.

1. Find the Cholesky decomposition A of $\Omega$.

2. Simulate 2 independent standard normal random variates $z_{1}$ and $z_{2}$.

3. Set $\mathbf{x}=A \mathbf{z}$.

4. Set $\mathrm{u}=\phi\left(x_{1}\right)$ and $\mathrm{v}=\phi\left(x_{2}\right)$, where $\phi$

denotes the univariate standard normal CDF.

5. The pair $(u, v)$ has the Gaussian copula as its distribution function.

In order to simulate a t-copula we use the relationship that if $S$ has a $\chi^{2}$ distribution and $\mathbf{Z}$ is the same as above, then

$$
\mathbf{X}={ }_{d} \mu+\frac{\sqrt{\nu}}{\sqrt{S}} \mathbf{Z}
$$

has the multivariate t-distribution with $\nu$ degrees of freedom. The first three steps of the algorithm 
are the same as for the Gaussian copula. The remaining steps are:

4. Simulate a random variate $\mathrm{s}$ from $\mathscr{X}_{\nu}^{2}$ independent of $z_{1}$ and $z_{2}$.

5. Set $\mathbf{y}=\frac{\sqrt{\nu}}{\sqrt{s}} \mathbf{x}$.

6. Set $\mathrm{u}=t_{\nu}\left(y_{1}\right)$ and $\mathrm{v}=t_{\nu}\left(y_{2}\right)$.

7. The pair $(u, v)$ has the t-copula as its distribution function.

\section{Simulation from Archimedean copulas}

The following results provide a basis for efficiently simulating Archimedean copulas. These results also find their application for testing the goodness-of-fit of copulas.

Theorem 2.3. Let $C$ be an Archimedean copula generated by $\varphi$ and let

$$
K_{C}(t)=V_{C}\left(\left\{(u, v) \in[0,1]^{2} \mid C(u, v) \leqslant t\right\}\right) .
$$

Then for any $t$ in $[0,1]$,

$$
K_{C}(t)=t-\frac{\varphi(t)}{\varphi^{\prime}\left(t^{+}\right)}
$$

Corollary 2.2. If $(U, V)$ has distribution function $C$, where $C$ is an Archimedean copula generated by $\varphi$, then the function $K_{C}$ is the distribution function of the random variable $C(U, V)$.

The theorem below allows for a direct implementation of the algorithm for simulating the copulas.

Theorem 2.4. Under the hypotheses of Corollary 2.2, the joint distribution function $H(s, t)$ if the random variables $S=\varphi(U) /[\varphi(U)+\varphi(V)]$ and $T=C(U, V)$ is given by $H(s, t)=s K_{C}(t)$ for all $(s, t)$ in $[0,1]^{2}$. Hence $S$ and $T$ are independent, and $S$ is uniformly distributed on $[0,1]$.

Now we are ready to present the algorithm:

1. Simulate two independent $U(0,1)$ random variates $s$ and $q$.

2. Set $t=K_{C}^{-1}(q)$, where $K_{C}$ is the distribution function of $C(U, V)$.

3. Set $\mathrm{u}=\varphi^{[-1]}(s \varphi(t))$ and $v=\varphi^{[-1]}((1-s) \varphi(t))$.

4. The desired pair is $(u, v)$. 


\subsection{Estimation and model selection}

When modeling the joint density of two random variables using copula functions, care needs to be taken as to how to correctly and efficiently estimate the parameters and how to discriminate between competing models. A number of methods for the estimation of copula functions has been described in the literature. However, when it is best to use one of the methods is not always an easy issue. For dealing with the issue of model selection a large number of tests of correct model specification of the marginals and the copula have been proposed.

\subsubsection{Estimation}

There exist five methods of estimating copula models. The one step method or exact maximum likelihood (EML) method estimates all parameters of the model at the same time. The second method is the two step estimator or the method of inference functions for margins (IFM), which first estimates the parameters of the marginals and conditional on these the parameters of the copula function are estimated. The canonical maximum likelihood method (CML), or the semiparametric estimation, leave the marginal densities unspecified and uses the empirical probability integral transform in order to obtain the uniform marginals needed to estimate the copula parameters. The last two methods are nonparametric ways of estimating the copula. The first one is estimating the empirical copula directly from the data, leaving the whole specification nonparametric. The other is obtaining a nonparametric estimate for Kendall's tau and using the relationship between Kendall's tau and the copula parameter to get an estimate of the latter. This method is due to Genest and Rivest (1993) and is mainly suited for Archimedean copulas.

\section{Exact maximum likelihood (EML)}

Let $\theta \in \Theta$ be the parameter vector to be estimated. This parameter vector can be split up into the parameters for the marginals and the copula function as follows $\theta=\left[\varphi^{\prime}, \gamma^{\prime}, \delta^{\prime}\right]^{\prime} . \varphi \in \phi$ denotes the parameter(s) of $f(x), \gamma \in \Gamma$ denotes the parameter(s) of $g(y)$, and $\delta \in \Delta$ denotes the parame$\operatorname{ter}(\mathrm{s})$ of $c(F(x), G(y))$. Assume we observe a sample $x_{t}$ and $y_{t}$ for $t=1, \ldots, T$. Consider the following representation of the joint density known as the copula decomposition of a joint distribution and the 
resulting log-likelihood function.

$$
\begin{aligned}
h\left(x_{t}, y_{t} ; \theta\right) & =f\left(x_{t} ; \varphi\right) \cdot g\left(y_{t} ; \gamma\right) \cdot c\left(F\left(x_{t} ; \varphi\right), G\left(y_{t} ; \gamma\right) ; \delta\right) \\
\mathscr{L}_{X Y} & =\sum_{t=1}^{T} \log \left(f\left(x_{t} ; \varphi\right)\right)+\sum_{t=1}^{T} \log \left(g\left(y_{t} ; \gamma\right)\right)+\sum_{t=1}^{T} \log \left(c\left(F\left(x_{t} ; \varphi\right), G\left(y_{t} ; \gamma\right) ; \delta\right)\right) \\
& =\mathscr{L}_{X}(\varphi)+\mathscr{L}_{Y}(\gamma)+\mathscr{L}_{C}(\varphi, \gamma, \delta)
\end{aligned}
$$

The ML estimator is then given by:

$$
\hat{\theta}=\arg \max \mathscr{L}_{X Y}
$$

This estimator is fully efficient as it attains the minimum asymptotic variance bound when the amount of data available for the two series is equal. However, it may be computationally difficult to obtain these estimates. Standard errors can be obtained in the usual way by the inverse of the Fisher information matrix.

\section{The parametric two-step estimator (IFM)}

This estimator makes use of the neat form of the copula decomposition of a joint distribution. In the first step the parameters $\varphi$ and $\gamma$ of the marginal densities are estimated by MLE, i.e.

$$
\begin{aligned}
& \hat{\varphi}=\arg \max \mathscr{L}_{X} \\
& \hat{\gamma}=\arg \max \mathscr{L}_{Y} .
\end{aligned}
$$

Using these estimates to transform the marginals into uniform $(0,1)$ variables one can estimate the copula parameter(s) $\delta$ by maximizing the copula density, i.e.

$$
\hat{\delta}=\arg \max \mathscr{L}_{C}(\hat{\varphi}, \hat{\gamma}, \delta) .
$$

Joe and Xu (1996) and Joe (2005) showed that this estimator is consistent and asymptotically normal. The asymptotic covariance matrix of $T^{1 / 2}(\hat{\theta}-\theta)$ is called the Godambe information matrix. If we denote the vector of score functions of our model by $\psi_{\theta}(X, Y)$ then it is given by $V=D^{-1} M\left(D^{-1}\right)^{\prime}$, where $D=E\left[\partial \psi_{\theta}(X, Y) / \partial \theta\right]$ and $M=E\left[\psi_{\theta}(X, Y) \psi_{\theta}(X, Y)^{\prime}\right]$. Joe and Xu (1996) suggest using the Jackknife method as an estimator for the covariance matrix and show its validity. If we denote the estimate of $\theta$ with the t'th row deleted from the data by $\theta^{(t)}$, then the Jackknife estimator of $T^{-1} V$ 
is

$$
\sum_{t}^{T}\left(\hat{\theta}^{(t)}-\hat{\theta}\right)\left(\hat{\theta}^{(t)}-\hat{\theta}\right)^{\prime} .
$$

In case the sample is large one may delete the data block wise and the approach remains valid.

The IFM approach has the advantage of being computationally less demanding than the EML approach. Furthermore, estimating the margins in a first step allows to assess the goodness of fit of the margins separately from that of the copula. Another advantage of this estimator is that it allows the two series $x_{t}$ and $y_{t}$ to be of unequal length. W.l.g. assume that the amount of data available for $X, T_{x}$, is greater than the amount of data available for $Y, T_{y}$. Then the additional information on the random variable $X$ can be used to find a better estimate of $\varphi$, which may lead to a better performance of the two-step estimator. Whether the use of this additional information offsets the loss of efficiency compared to the one-step estimator depends on the ratios $\frac{T_{y}}{T_{x}} \equiv \lambda_{y}$ and $\frac{T_{c}}{T_{x}} \equiv \lambda_{c}$, where $T_{c}$ is the number of observations available for the estimation of the copula, $0<\lambda_{y}<1$ and $0<\lambda_{c}<1$.

Patton (2006a) proposes a one-step adjustment of this estimator, that theoretically makes it fully efficient. However, in a Monte Carlo study the small sample properties of this adjusted estimator are shown to be rather poor. In contrast, the unadjusted estimator outperforms the EML estimator in the setup chosen.

\section{The semi parametric two-step estimator (CML)}

When the densities of the marginal distributions are unknown this estimator allows for the possibility of leaving them unspecified. Bad estimation results due to miss specification of the marginals can be avoided. In the first step the series of interest are transformed into uniform variates using the empirical probability integral transform. The empirical distribution function is defined as

$$
\hat{F}(\cdot)=\frac{1}{T} \sum_{t=1}^{T} \mathbf{1}_{\left\{X_{t} \leqslant \cdot\right\}},
$$

where $\mathbf{1}_{\left\{X_{t} \leqslant\right\}}$ is the indicator function. The copula parameter $\delta$ can then be estimated by maximizing the log-likelihood function of the copula density using the transformed variables given by

$$
\mathscr{L}_{C}(\delta)=\sum_{t=1}^{T} \log \left(c\left(\hat{F}\left(x_{t}\right), \hat{G}\left(y_{t}\right)\right)\right)=\sum_{t=1}^{T} \log \left(c\left(\hat{u}_{t}, \hat{v}_{t}\right) .\right.
$$


The semi parametric estimator then is

$$
\hat{\delta}=\arg \max \mathscr{L}_{C}(\delta)
$$

Consistency and asymptotic normality of the CML estimator were shown by Genest, Ghoudi, and Rivest (1995) for the i.i.d. case and by Chen and Fan (2006b) for estimating copula based time series models. Chen and Fan (2005) showed that this estimator also converges to the pseudo true parameter in case the copula is misspecified (which in most practical situations is very likely), so that the estimated model is closest to the data generating process in terms of the Kullback-Leibler divergence.

Estimators for the covariance matrix are described by the authors for the various cases. For a clear step by step procedure for the i.i.d. case we refer to Genest and Favre (2007).

\section{The nonparametric estimator}

Unlike the methods described above this method does not rely on any parametric specification of the copula function. The empirical copula is the function $C_{n}$ given by

$$
C_{n}\left(\frac{i}{T}, \frac{j}{T}\right)=\frac{\text { number of pairs }\left(x_{t}, y_{t}\right) \text { in the sample such that } x_{t} \leqslant x_{(i)} \text { and } y_{t} \leqslant y_{(j)}}{T},
$$

for $i, j=1, \ldots, T$, where $x_{(i)}$ and $y_{(j)}$ are the $i$ th and $j$ th order statistics of $x$ and $y$. The empirical copula can be used to calculate population versions of the concordance measure described above. For more details on that see Nelsen (2006). Additionally, they find their use in nonparametric tests for independence and for goodness of fit test of the copula specification. Note that Fermanian and Scaillet (2003) alternatively propose a kernel estimator for copulas.

\section{The nonparametric estimator by Genest and Rivest (1993)}

An advantage of this approach is that the marginal distributions do not need to be specified. If we let $\mathrm{c}$ denote the number of concordant pairs in the sample and d the number of discordant pairs in the sample, then the sample version of Kendall's tau is given by

$$
\hat{\tau}=\frac{c-d}{c+d}
$$


Using this estimate and the relationship between the copula parameter and Kendall's tau the nonparametric estimate can be obtained easily if a closed form expression for this relationship exists. In case a closed form expression does not exists one can still estimate the copula parameter by using the general form of Kendall's tau for Archimedean copulas. This takes the form

$$
\tau_{C}=1+4 \int_{0}^{1} \frac{\varphi(t)}{\varphi^{\prime}(t)} d t
$$

and might be solved using a computer program like Mathematica or Maple. An obvious drawback of this estimator is that it only applies to the limited number of one parameter models. When it is available one may compare the parameter estimate of the copula model to the estimate obtained by a MLE for a first check of its goodness-of-fit. When the two estimates are close to each other one has an indication of a reasonable fit.

\subsubsection{Model selection}

Once one or more estimates of a certain parametric copula have been obtained a very important issue is how to compare the competing models. The first thing to be done is to assess the goodness-of-fit of the marginal distribution. Both the i.i.d.'ness and the correct specification of the distribution need to be tested. For testing the i.i.d.'ness we refer to Diebold, Gunther, and Tay (1998) who proposed a simple procedure for this task. The specification of the distribution can be tested by testing the transformed series for uniformity. This can be done by using the well known Kolmogorov-Smirnov test or a $\chi^{2}$ test.

A huge number of tests have been proposed for testing the copula specification. Examples are Chen, Fan, and Patton (2008), Chen and Fan (2005), Genest, Quessy, and Rémillard (2006) and Fermanian (2005). However, none of these tests has proven to be superior and quite some research remains to be done in this field. Besides the numerous goodness-of-fit tests there are a few model selection criteria, which allow to rank the copulas according to their fit in some way. The most widely used criterium is the Akaike information criterion. It is defined as

$$
A I C=2(\text { negative log-likelihood })+2 k
$$

where $k$ is the number of parameters in the model. The model with the lowest AIC should be considered as the best fitting one. 


\section{Graphical methods}

The first step we propose in the model selection process is a visual inspection of the scatterplot of the data. Some dependence structures may already be identified such as dependence in one of the tails. However, there are more formal ways of assessing the goodness-of-fit of your model by visual means. Consider the conditional distribution function(d.f.) of $Y$ given $X$. It is given by

$$
H_{Y \mid X}(x, y)=C(G(y) \mid F(x))
$$

where $C(v \mid u)=\frac{\partial}{\partial u} C(u, v)$. The conditional d.f. is $U(0,1)$ distributed, if the joint density is well specified. Therefore a QQ-plot of the conditional d.f. using the estimated parameter and the observed data $x$ and $y$ against uniform quantiles should yield a straight line.

A similar method makes use of Theorem 2.3 and Corollary 2.2, which state that the distribution function of the copula can be represented in the following way:

$$
K_{C}(t)=P[C(U, V) \leqslant t]=t-\frac{\varphi(t)}{\varphi^{\prime}\left(t^{+}\right)}
$$

Therefore $K_{C}(C(F(X), G(Y))$ should be uniformly distributed, so in a similar fashion as above the QQ-Plot can be constructed and can be compared to a straight line.

A third method, which was proposed by Genest and Rivest (1993), relies on two estimates for the distribution function of the copula $K_{C}$. One is a parametric estimate obtained by MLE, the other is the empirical copula, which is determined by the method described above. A QQ-Plot of these two distribution function should help determine the best fitting copula. For a good exposition of this method, possible drawbacks, and some applications the reader is referred to de Matteis (2001). Note that again we can also look at the histogram of the d.f. of interest and compare it to the histogram of a $U(0,1)$ variate. The histogram should be close to a horizontal line under a good specification.

\section{Tests based on the graphical methods}

The idea that both the conditional d.f. and the d.f. of the copula should follow a $U(0,1)$ distribution under a correct specification of the copula can also be used to conduct formal tests. de Matteis (2001) (among others) proposes to apply the Kolmogorov-Smirnov (K-S) test and the $\chi^{2}$ test to check the null hypothesis of a $U(0,1)$ distribution. We suggest additionally using the Jarque-Bera test after 
transforming the data through the inverse CDF of the normal distribution.

As mentioned above there are many more tests available for testing the goodness-of-fit of the copula specification. However, we postpone a systematic comparison.

\subsection{Simulation study}

So far the different estimators and some simple model selection tests for copula functions have been introduced. However, small sample performance of both the different estimators and the testing procedures are not always obvious. In order have a better idea on how to properly model data using copulas Monte Carlo experiments will be performed. The setup of the simulations are supposed to resemble decisions that have to be made by a researcher working on practical issues. This could include issues like which estimation technique to use, which copula functions to consider to describe certain dependence structures, small sample power of a certain test, behavior of competing tests under misspecification, etc. In the copula literature Monte Carlo studies are frequently performed, but often attention is only paid to testing the power of a certain test under a very limited number of data generating processes, as a general setting is not always possible. A problem is that most competing copula models are non-nested. Therefore, a lot of questions remain open and it is easy to set up interesting studies. Here we will consider two different simulation studies. In the first the properties of the different estimation techniques are compared, both when the density of the marginals is known and when it is misspecified. In the second an attempt is made to compare the methods for model selection for competing copula models we presented above.

\subsubsection{Comparison of the estimators}

In this section we want to take a closer look at the properties of the estimator that have been suggested in the literature. Theoretically their properties have been well studied and we expect to confirm the theoretical findings. We consider the following estimators: EML, IFM, CML and the nonparametric estimator based on Kendall's tau, which we shall call method of moments (MM) here. The data generating process is a rather simple one. Data is simulated from a Clayton copula with its parameter corresponding to Kendall's tau equal to 0.2, 0.4, 0.6 and 0.8. As marginal distributions we took t-distributions with 5 and 6 degrees of freedom for the $X$ and $Y$ series, respectively. Formally, 
Table 2.1: Mean Square Errors for different estimators

\begin{tabular}{rrrrrrrr}
\hline \hline $\mathrm{T}=100$ & $\tau$ & EML & IFM & CML & MM & EML MS & IFM MS \\
\hline & 0.2 & 0.0369 & 0.003 & 0.0038 & 0.0044 & 0.0223 & 0.0043 \\
& 0.4 & 0.0022 & 0.0023 & 0.0031 & 0.0039 & 0.0039 & 0.0035 \\
& 0.6 & 0.001 & 0.0013 & 0.0018 & 0.0023 & 0.0021 & 0.0019 \\
& 0.8 & 0.0003 & 0.0009 & 0.0008 & 0.0008 & 0.0007 & 0.0016 \\
$\mathrm{~T}=250$ & & & & & & & \\
\hline & 0.2 & 0.0056 & 0.0012 & 0.0014 & 0.0017 & 0.0141 & 0.0022 \\
& 0.4 & 0.0008 & 0.0008 & 0.0011 & 0.0014 & 0.0014 & 0.0013 \\
& 0.6 & 0.0004 & 0.0005 & 0.0007 & 0.0008 & 0.001 & 0.0007 \\
& 0.8 & 0.0001 & 0.0003 & 0.0003 & 0.0003 & 0.0002 & 0.0005 \\
$\mathrm{~T}=500$ & & & & & & & \\
\hline & 0.2 & 0.0009 & 0.0006 & 0.0007 & 0.0008 & 0.0065 & 0.0016 \\
& 0.4 & 0.0004 & 0.0004 & 0.0006 & 0.0007 & 0.0008 & 0.0007 \\
& 0.6 & 0.0002 & 0.0002 & 0.0003 & 0.0004 & 0.0006 & 0.0004 \\
& 0.8 & 0.0001 & 0.0001 & 0.0001 & 0.0001 & 0.0004 & 0.0003 \\
$\mathrm{~T}=1000$ & & & & & & & \\
\hline & 0.2 & 0.0003 & 0.0003 & 0.0003 & 0.0004 & 0.0031 & 0.0012 \\
& 0.4 & 0.0002 & 0.0002 & 0.0003 & 0.0003 & 0.0004 & 0.0004 \\
& 0.6 & 0.0001 & 0.0001 & 0.0002 & 0.0002 & 0.0004 & 0.0002 \\
& 0.8 & 0.00002 & 0.00004 & 0.0001 & 0.0001 & 0.0011 & 0.0005 \\
\hline \hline
\end{tabular}

Note: The table reports the MSE from the implied Kendall's tau to the true one. Data is generated from a Clayton copula with Student t margins. EML MS and IFM MS refer to the estimator when the margins are assumed to be normally distributed.

we have

$$
\begin{aligned}
(u, v) & \sim C_{\delta}^{\text {Clayton }} \\
X & =t^{-1}(u ; 5) \\
Y & =t^{-1}(v ; 6) .
\end{aligned}
$$

The sample sizes we look at are 100, 250, 500 and 1000 observations. All simulations will be replicated 1000 times. We only focus on the estimation of the copula parameter, although one has to keep in mind that for the EML and IFM estimators additionally the degrees of freedom parameter needs to be estimated. We use these two estimators both in the situation when the marginals are known to follow a t-distribution and when they are wrongly specified, which is a situation that is highly relevant in practice. In particular, normal marginals are estimated in the misspecified case. For the CML and the MM estimators, of course, the marginals remain unspecified. Theoretically the EML estimator is the most efficient one, followed by the IFM estimator and the CML estimator, but it remains to be 
seen whether this also holds in our simulation setup and how large the gain in efficiency is. Another interesting issue is how the CML and MM estimators compare to the misspecified estimators, because it can provide a guideline as to which estimator to use whenever one is not sure about the specification of the marginal distributions.

Table 2.1 reports the Mean Square Errors (MSE) of the estimates for Kendall's tau corresponding to the estimated copula parameter and its true value. We decided to compute the MSE for Kendall's tau and not for the copula parameter itself, because that allows comparison of the efficiency over different degrees of dependence. Overall the EML estimator is indeed superior to the others, but for weak dependence structures the IFM method is actually better and the difference between the two is rather small. For correctly specified models the MM estimator is the worst. The CML estimator is, as expected, not as efficient as the fully parametric methods. Only for tau equal to 0.2 and $T$ less than 1000 does it outperform the EML method. When the marginals are misspecified the IFM estimator is clearly superior to the EML estimator. However, it is almost always worse than the semi-parametric estimator. Thus, whenever one fears that the marginals are misspecified we recommend using the CML method. In case a fully parametric estimate is needed (e.g. because the behavior of some test statistic is not known for a semi-parametric estimator) we recommend using the IFM estimator. Note that as the sample size and the degree of dependence increase the MSE's greatly decrease.

\subsubsection{Model selection}

A typical problem that arises when fitting copulas to data is how to decide for the best fitting model. A large number of methods has been proposed for that task. Here we compare the ones we proposed earlier, namely the AIC and three tests for checking whether the conditional copula is distributed $U(0,1)$, which are the K-S test, the $\chi^{2}$ test $^{3}$ and the Jarque-Bera test. In our first simulation we sample data from three different copulas, the Gumbel, Clayton and Frank copulas with $U(0,1)$ marginals and parameters corresponding to Kendall's tau equal to 0.2, 0.4 and 0.6 and sample sizes of 100, 250, 500 and 1000 observations. Then we estimate the following copulas: Gumbel, survival Gumbel, Clayton, survival Clayton, Frank, Plackett and Normal copula, which are the most popular one-parameter copulas in the literature. Again we use 1000 replications for the Monte Carlo simulations.

Tables 2.2-2.4 report the fraction of times each candidate model is chosen when using a selection rule the always decides for the model with the smallest AIC. This model selection procedure becomes

\footnotetext{
${ }^{3}$ For the $\chi^{2}$ test we use 15, 20, 30 and 50 classes for 100, 250, 500 and 1000 observations, respectively.
} 
Table 2.2: Model selection by AIC when the true model is the Gumbel copula

\begin{tabular}{ccccccccc}
\hline \hline $\mathrm{T}=100$ & $\tau$ & Gumbel & rot. Gumbel & Clayton & rot. Clayton & Frank & Plackett & Normal \\
\hline & 0.2 & 0.333 & 0.037 & 0.015 & 0.373 & 0.053 & 0.077 & 0.112 \\
& 0.4 & 0.588 & 0.009 & 0 & 0.227 & 0.023 & 0.044 & 0.109 \\
& 0.6 & 0.806 & 0 & 0 & 0.09 & 0.019 & 0.019 & 0.066 \\
$\mathrm{~T}=250$ & & & & & & & & \\
\hline & 0.2 & 0.54 & 0.008 & 0 & 0.277 & 0.031 & 0.056 & 0.088 \\
& 0.4 & 0.844 & 0 & 0 & 0.113 & 0.004 & 0.008 & 0.031 \\
& 0.6 & 0.978 & 0 & 0 & 0.016 & 0 & 0 & 0.006 \\
$\mathrm{~T}=500$ & & & & & & & & \\
\hline & 0.2 & 0.731 & 0 & 0 & 0.205 & 0.013 & 0.012 & 0.039 \\
& 0.4 & 0.95 & 0 & 0 & 0.046 & 0 & 0.001 & 0.003 \\
$\mathrm{~T}=1000$ & 0.6 & 0.998 & 0 & 0 & 0.001 & 0 & 0 & 0.001 \\
& & & & & & & & \\
\hline & 0.2 & 0.856 & 0.001 & 0 & 0.125 & 0.001 & 0.002 & 0.015 \\
& 0.4 & 0.99 & 0 & 0 & 0.01 & 0 & 0 & 0 \\
& 0.6 & 1 & 0 & 0 & 0 & 0 & 0 & 0 \\
\hline \hline
\end{tabular}

more powerful with stronger dependence and larger samples sizes. In most of the cases when the true model is not selected the model with the lowest AIC is one with similar dependence structure. For example both the Gumbel and the rotated Clayton copula are characterized by upper tail dependence and one can see that when the data is generated from a Gumbel copula the rotated Clayton copula is selected quite often. Altogether one can say that the AIC is a good criterion for finding the best fitting copula. However, for weak dependence its performance is not entirely satisfactory. The good news is that when it does not find the correct model it will usually chose one that is close to it.

In order to get an idea about the size and the power under different alternatives of the three goodness-of-fit tests we consider the problem of testing whether data has been generated by a Gaussian copula. To this end we draw random observations from the Gaussian copula, to check the size of the tests, and from two alternatives, namely the t-copula with 4 degrees of freedom and the Clayton copula. The t-copula is chosen to see the behavior of the test when the alternative is also symmetric, but has fatter tails than then null. The Clayton copula, on the other hand, has an asymmetric dependence structure and we expect the tests to have higher power against this alternative.

In Tables 2.5-2.7 the rejection frequencies at a level of 5\% for the three mentioned tests are given. First note that all three tests have a higher power against the Clayton copula as the alternative than against the t-copula. In terms of size the K-S test performs quite well. It seems to be slightly undersized. Its power, however, is only acceptable for strong dependence structures and large samples. The $\chi^{2}$ test is clearly oversized, but its power is a lot higher than for the K-S test. Finally, the JarqueBera test has both good size properties and it is superior to the other two tests in terms of power. 
Table 2.3: Model selection by AIC when the true model is the Clayton copula

\begin{tabular}{ccccccccc}
\hline \hline $\mathrm{T}=100$ & $\tau$ & Gumbel & rot. Gumbel & Clayton & rot. Clayton & Frank & Plackett & Normal \\
& 0.2 & 0.01 & 0.192 & 0.647 & 0.001 & 0.041 & 0.033 & 0.076 \\
& 0.4 & 0 & 0.153 & 0.829 & 0 & 0.01 & 0.001 & 0.007 \\
& 0.6 & 0 & 0.07 & 0.93 & 0 & 0 & 0 & 0 \\
$\mathrm{~T}=250$ & & & & & & & & \\
\hline & 0.2 & 0.001 & 0.205 & 0.735 & 0 & 0.019 & 0.013 & 0.027 \\
& 0.4 & 0 & 0.085 & 0.915 & 0 & 0 & 0 & 0 \\
& 0.6 & 0 & 0.008 & 0.992 & 0 & 0 & 0 & 0 \\
$\mathrm{~T}=500$ & & & & & & & & 0.002 \\
$\mathrm{~T}=1000$ & 0.2 & 0 & 0.185 & 0.808 & 0 & 0 & 0 & 0.005 \\
& 0.4 & 0 & 0.021 & 0.979 & 0 & 0 & 0 & 0 \\
& 0.6 & 0 & 0.002 & 0.998 & 0 & 0 & 0 & 0 \\
& 0.2 & 0 & 0.092 & 0.908 & 0 & 0 & 0 & 0 \\
& 0.4 & 0 & 0.002 & 0.998 & 0 & 0 & 0 & 0 \\
& 0.6 & 0 & 0 & 1 & 0 & 0 & 0 & 0 \\
\hline \hline
\end{tabular}

Thus the Jarque-Bera test is the most recommendable test of the three. This may be due to the fact that it is able to detect skewness and excess kurtosis, which in terms of copulas means it is able to reveal whether a model insufficiently captures asymmetric dependence or fat tails. Still one can see that the performance of the tests is not acceptable for weak dependence structures, even when the sample is large. Thus we conclude that these tests are not very useful in small samples and for weak dependence. They are powerful only in situations when model selection using the AIC works extremely well. The K-S test is the weakest of the three and it should be interpreted with care. The $\chi^{2}$ and especially the Jarque-Bera test can be useful for complementing model selection by the AIC. Furthermore, formal testing is necessary, since one cannot be sure that the true model is among the ones selected for estimation. Thus, even when a model clearly outperforms its competitors in terms of AIC none of the estimated copulas may have a satisfactory fit.

\subsection{Modeling exchange rate dependence}

Now that we have introduced copula functions, the different ways of estimating them for a given data set and some model specification tests, including an analysis of their finite sample properties, we are ready to present their use in practice. We use copulas to model the dependence between pairs of Latin American exchange rates against the Euro. In particular we consider daily exchange rate returns from $01 / 01 / 2001$ until 30/11/2006, which amounts to 1485 observations for each series, for 
Table 2.4: Model selection by AIC when the true model is the Frank copula

\begin{tabular}{ccccccccc}
\hline \hline $\mathrm{T}=100$ & $\tau$ & Gumbel & rot. Gumbel & Clayton & rot. Clayton & Frank & Plackett & Normal \\
\hline & 0.2 & 0.067 & 0.086 & 0.085 & 0.077 & 0.319 & 0.188 & 0.178 \\
& 0.4 & 0.038 & 0.04 & 0.015 & 0.009 & 0.567 & 0.204 & 0.127 \\
& 0.6 & 0.013 & 0.01 & 0 & 0.002 & 0.817 & 0.106 & 0.052 \\
$\mathrm{~T}=250$ & & & & & & & & \\
\hline & 0.2 & 0.044 & 0.031 & 0.029 & 0.031 & 0.433 & 0.261 & 0.171 \\
& 0.4 & 0.008 & 0.003 & 0 & 0 & 0.756 & 0.151 & 0.082 \\
& 0.6 & 0 & 0.001 & 0 & 0 & 0.949 & 0.043 & 0.007 \\
$\mathrm{~T}=500$ & & & & & & & & \\
\hline & 0.2 & 0.005 & 0.015 & 0.003 & 0.003 & 0.526 & 0.32 & 0.128 \\
& 0.4 & 0 & 0.002 & 0 & 0 & 0.843 & 0.135 & 0.02 \\
$\mathrm{~T}=1000$ & 0.6 & 0 & 0 & 0 & 0 & 0.99 & 0.009 & 0.001 \\
& & & & & & & & \\
\hline \hline & 0.2 & 0.001 & 0.002 & 0.001 & 0 & 0.612 & 0.32 & 0.064 \\
& 0.4 & 0 & 0 & 0 & 0 & 0.946 & 0.053 & 0.001 \\
& 0.6 & 0 & 0 & 0 & 0 & 1 & 0 & 0 \\
\hline \hline
\end{tabular}

Table 2.5: Rejection frequencies for the KS-test

\begin{tabular}{rrrrrrrr}
\hline \hline $\mathrm{T}=100$ & Normal & Student & Clayton & $\mathrm{T}=250$ & Normal & Student & Clayton \\
$\tau=0.2$ & 0.053 & 0.036 & 0.05 & & 0.049 & 0.052 & 0.057 \\
$\tau=0.4$ & 0.056 & 0.053 & 0.096 & & 0.04 & 0.056 & 0.15 \\
$\tau=0.6$ & 0.041 & 0.054 & 0.184 & & 0.031 & 0.088 & 0.458 \\
\hline $\mathrm{T}=500$ & & & & $\mathrm{~T}=1000$ & & & \\
$\tau=0.2$ & 0.034 & 0.051 & 0.068 & & 0.06 & 0.057 & 0.108 \\
$\tau=0.4$ & 0.038 & 0.07 & 0.35 & & 0.053 & 0.1 & 0.567 \\
$\tau=0.6$ & 0.043 & 0.182 & 0.804 & & 0.051 & 0.41 & 0.999 \\
\hline \hline
\end{tabular}

Note: Data is generated from a Gaussian copula, a t-copula with 4 degrees of freedom and a Clayton copula with $U(0,1)$ margins. The table reports the frequency of rejection of tests of uniformity of the conditional Gaussian copula $C_{\text {Gaussian }}(u \mid v)$.

the following countries: Brazil, Chile, Columbia, Mexico and Peru. ${ }^{4}$

All the results on estimation and model selection require i.i.d. $U(0,1)$ observations. This requires a first estimation step to filter out mean dynamics and heteroscedasticity. Autocorrelation or an incorrect specification of the marginal distribution can influence the estimation of the copula parameters. The first step in modeling the data is to model the marginal distribution of each series individually in order to transform them into i.i.d. $U(0,1)$ series. To this end we start by fitting ARMA models to each series and then fitting GARCH models to their residuals. The mean dynamics where captured well by only an intercept for all series except Mexico. In that case a simple AR(1) model provided a good fit for the data. The best fit for the conditional variance was a t-GARCH $(1,1)$ for

\footnotetext{
${ }^{4}$ The data have been taken from the internet page of the PACIFIC Exchange Rate Service provided by the University of British Columbia (http://fx.sauder.ubc.ca/data.html).
} 
Table 2.6: Rejection frequencies for the $\chi^{2}$ test

\begin{tabular}{rrrrlrrr}
\hline \hline $\mathrm{T}=100$ & Normal & Student & Clayton & $\mathrm{T}=250$ & Normal & Student & Clayton \\
$\tau=0.2$ & 0.1 & 0.091 & 0.093 & & 0.073 & 0.1 & 0.106 \\
$\tau=0.4$ & 0.079 & 0.084 & 0.162 & & 0.077 & 0.099 & 0.301 \\
$\tau=0.6$ & 0.058 & 0.108 & 0.354 & & 0.078 & 0.139 & 0.74 \\
\hline $\mathrm{T}=500$ & & & & $\mathrm{~T}=1000$ & & & \\
$\tau=0.2$ & 0.083 & 0.076 & 0.117 & & 0.077 & 0.069 & 0.139 \\
$\tau=0.4$ & 0.071 & 0.105 & 0.517 & & 0.065 & 0.135 & 0.772 \\
$\tau=0.6$ & 0.076 & 0.237 & 0.959 & & 0.081 & 0.414 & 1 \\
\hline \hline
\end{tabular}

Note: Data is generated from a Gaussian copula, a t-copula with 4 degrees of freedom and a Clayton copula with $U(0,1)$ margins. The table reports the frequency of rejection of tests of uniformity of the conditional Gaussian copula $C_{\text {Gaussian }}(u \mid v)$.

Table 2.7: Rejection frequencies for the JB-test

\begin{tabular}{rrrrlrrr}
\hline \hline $\mathrm{T}=100$ & Normal & Student & Clayton & $\mathrm{T}=250$ & Normal & Student & Clayton \\
$\tau=0.2$ & 0.061 & 0.08 & 0.083 & & 0.053 & 0.149 & 0.122 \\
$\tau=0.4$ & 0.05 & 0.301 & 0.344 & & 0.048 & 0.495 & 0.65 \\
$\tau=0.6$ & 0.055 & 0.496 & 0.706 & & 0.054 & 0.796 & 0.965 \\
\hline $\mathrm{T}=500$ & & & & $\mathrm{~T}=1000$ & & & \\
$\tau=0.2$ & 0.059 & 0.166 & 0.236 & & 0.057 & 0.257 & 0.439 \\
$\tau=0.4$ & 0.053 & 0.725 & 0.928 & & 0.056 & 0.936 & 0.998 \\
$\tau=0.6$ & 0.044 & 0.954 & 1 & & 0.05 & 0.993 & 1 \\
\hline \hline
\end{tabular}

Note: Data is generated from a Gaussian copula, a t-copula with 4 degrees of freedom and a Clayton copula with $U(0,1)$ margins. The table reports the frequency of rejection of tests of uniformity of the conditional Gaussian copula $C_{\text {Gaussian }}(u \mid v)$.

all series. The standardized residuals where fit to a Student t-distribution using maximum likelihood estimation (MLE) and transformed into $U(0,1)$ variables using the CDF of the t-distribution and the estimated degrees of freedom parameter. The uniformity is tested using the tests also proposed for the goodness-of-fit tests for copulas, the K-S test, the $\chi^{2}$ test and the Jarque-Bera test. None of the series passed all three tests, so the uniformity assumption is questionable. Therefore we modeled the standardized residuals with the skewed t-distribution introduced by Hansen (1994). The p-values of the goodness-of-fit a given in Table 2.8. ${ }^{5}$ All series fit the skewed t-distribution quite well so we can continue working with the transformed series. Note that the skewness parameters were quite small (below 0.1 in absolute values) for all series.

The second step is actually estimating a number of copulas and deciding which is the best fitting one. The estimation is done using the IFM method, since we have already modeled the marginals

\footnotetext{
${ }^{5}$ Note that we used 55 classes for the $\chi^{2}$ test.
} 
Table 2.8: Goodness-of-fit tests for marginal distributions

\begin{tabular}{rrrrrr}
\hline \hline & Bra & Chi & Col & Mex & Peru \\
\hline KS & 0.6361 & 0.5455 & 0.8814 & 0.5246 & 0.5465 \\
$\chi^{2}$ & 0.2486 & 0.5941 & 0.3247 & 0.8275 & 0.0829 \\
JB & 0.9044 & 0.8643 & 0.9424 & 0.979 & 0.9237 \\
\hline \hline
\end{tabular}

Note: The series have been fit to a skewed t-distribution and were transformed by the skewed t CDF.

Table 2.9: Kendall's tau matrix between the GARCH residuals

\begin{tabular}{rrrrrr}
\hline \hline & bra & chi & col & mex & peru \\
\hline bra & 1 & 0.4513 & 0.3602 & 0.4346 & 0.3649 \\
chi & 0.4513 & 1 & 0.4299 & 0.5129 & 0.4727 \\
col & 0.3602 & 0.4299 & 1 & 0.5018 & 0.5721 \\
mex & 0.4346 & 0.5129 & 0.5018 & 1 & 0.5785 \\
peru & 0.3649 & 0.4727 & 0.5721 & 0.5785 & 1 \\
\hline \hline
\end{tabular}

and we found that IFM estimator behaves quite well compared to the one step estimator. An argument for using the EML technique is that it is easier to get the standard errors, but we are not interested in testing any hypothesis about the parameters. We also estimated all the models with the semi-parametric estimator, but the results were very close to the fully parametric approach, so we report only the outcomes for the latter estimator. To get an idea of the degree of dependence between the series the Kendall's tau matrix is given in Table 2.9. It ranges from 0.36 for the pair Brazil-Columbia until 0.57 for Peru-Mexico. The copulas we estimate are the following: Gumbel, survival Gumbel, Clayton, survival Clayton, Frank, Plackett, Normal, Student, Joe-Clayton(JC), symmetrized JC, BB1, mixture Clayton-Gumbel, mixture Clayton-Frank, mixture Gumbel-Frank, mixture Gumbel-survival Gumbel, mixture Clayton-survival Clayton and mixture JC-Frank. All copulas are estimated and the models were ranked according to the AIC. Then the Jarque-Bera test on the conditional copula is performed (after transforming the series with the inverse Gaussian CDF as before). The test was performed both for the conditional copula $\mathrm{U}$ given $\mathrm{V}$ and $\mathrm{V}$ given $\mathrm{U}$, so the derivative of the copula distribution function with respect to each of its arguments. The highest ranked model that passed the Jarque-Bera test in both directions is considered the best fitting one. In some cases, however, models were very close to each other in terms of the AIC, so deciding for the best fitting one is a rather hard task. More refined model selection techniques may be necessary in this case. In such situations we simply report more than one model. Overall there are three dominant copulas, the Student copula, the Joe-Clayton-Frank mixture and the mixture Gumbel- 
Table 2.10: Estimation results

\begin{tabular}{|c|c|c|c|c|c|c|c|}
\hline Countries & Model & $\delta_{1}$ & $\delta_{2}$ & $\alpha$ & $\lambda_{U}$ & $\lambda_{L}$ & $\mathrm{AIC}$ \\
\hline \multicolumn{8}{|l|}{ bra-chi } \\
\hline & JC-Frank & $1.6609 ; 0.5369$ & 6.2095 & 0.5173 & 0.2494 & 0.1423 & -787.169 \\
\hline & Student & 0.6411 & 12.1674 & - & 0.1132 & 0.1132 & -782.364 \\
\hline \multirow[t]{4}{*}{ bra-col } & & & & & & & \\
\hline & Gumbel- rot. Gumbel & 1.5091 & 1.655 & 0.6843 & 0.2854 & 0.1515 & -497.262 \\
\hline & Clayton-Gumbel & 1.3378 & 1.5201 & 0.2182 & 0.3301 & 0.1299 & -495.439 \\
\hline & Gumbel-Frank & 1.4721 & 4.6115 & 0.6315 & 0.2518 & 0 & -495.059 \\
\hline \multirow[t]{3}{*}{ bra-mex } & & & & & & & \\
\hline & JC-Frank & $1.6302 ; 0.5742$ & 5.4081 & 0.4498 & 0.2115 & 0.1345 & -718.688 \\
\hline & Student & 0.6214 & 10.1643 & - & 0.1343 & 0.1343 & -716.062 \\
\hline \multirow[t]{3}{*}{ bra-peru } & & & & & & & \\
\hline & Student & 0.5344 & 7.5576 & - & 0.1433 & 0.1433 & -502.37 \\
\hline & Gumbel- rot. Gumbel & 1.4132 & 1.8116 & 0.5623 & 0.2063 & 0.2337 & -499.56 \\
\hline chi-col & Gumbel- rot. Gumbel & 1.4722 & 2.1164 & 0.4516 & 0.18 & 0.3359 & -758.907 \\
\hline \multirow[t]{2}{*}{ chi-mex } & & & & & & & \\
\hline & Student & 0.719 & 14.1568 & - & 0.1361 & 0.1361 & -1080.69 \\
\hline \multirow[t]{2}{*}{ chi-peru } & & & & & & & \\
\hline & Student & 0.6763 & 5.1767 & - & 0.3156 & 0.3156 & -943.031 \\
\hline \multirow[t]{4}{*}{ col-mex } & & & & & & & \\
\hline & JC-Frank & $1.9722 ; 1.2787$ & 5.4311 & 0.4904 & 0.2839 & 0.2852 & -1002.99 \\
\hline & Gumbel- rot. Gumbel & 1.8917 & 2.102 & 0.5009 & 0.2793 & 0.3041 & -1002.41 \\
\hline & Clayton-Gumbel & 2.4352 & 1.9038 & 0.3166 & 0.3832 & 0.2382 & -999.295 \\
\hline col-peru & Cumbl rot Cumbol & 16847 & 31245 & 0.3706 & 0182 & 04736 & -143948 \\
\hline \multirow[t]{2}{*}{ mex-peru } & & & & & & & \\
\hline & Student & 0.781 & 12.9949 & - & 0.2107 & 0.2107 & -1392.82 \\
\hline
\end{tabular}

Note: Estimation results for the best fitting copula models obtained by the IFM method for standardized GARCH residuals. In case of mixtures $\delta_{1}$ is the estimate of the first model (two parameters in the JC-Frank copula), $\delta_{2}$ is the estimate of the second model and $\alpha$ is the mixing parameter.

survival Gumbel. Furthermore, it is apparent that all pairs of exchange rates exhibit tail dependence in both tails. Models that do not allow for dependence in both tails are not among the best fitting ones. We make the same observation as Junker et al. (2005) that a one parameter copula is not able to provide a reasonable fit for financial data.

For the pairs Brazil-Chile, Brazil-Columbia and Brazil-Mexico we observe an asymmetric dependence structure with more upper tail dependence than lower tail dependence. This means that when these currencies depreciate against the Euro they tend to be more dependent that when they appreciate. However, for the pair Brazil-Mexico the evidence is not that strong and dependence may actually be symmetric. The pairs Chile-Columbia, Columbia-Peru also have an asymmetric dependence structure, but they have more lower tail dependence than upper tail dependence. For the rest of the pair we can conclude that dependence is rather symmetric. Only for the pair Columbia-Mexico is there some evidence for excess upper tail dependence. Our findings are in line with the findings of 
Patton (2006b) and Patton (2006a) who finds asymmetric dependence for the Euro/German Mark and the Yen against the dollar. Note that in Patton (2006b) it is found that exchange rate dependence may be varying over time and should therefore be modeled using conditional copula models. The treatment of time-varying copula models can be found in later chapters.

\subsection{Conclusion}

In this chapter we introduced copula functions both in a formal and practical manner. We established some important links between copulas and some common measures of dependence, after presenting the relevant dependence concepts, in order to enable the reader to interpret these measures in a correct way. The introduction of the most commonly used examples and families of copulas, together with the different estimators and some goodness-of-fit tests, gave us all the tools necessary to model a data set. However, the theory is not able to give any guidelines as to which copula functions to consider, which estimator to use or how to discriminate between competing models. Providing some guidelines to these issues turned out to be the main point of this work. With the help of Monte Carlo simulations we can recommend the use of the CML estimator whenever a fully parametric model is not needed and when one runs the danger of working with misspecified marginal distributions. When one is confident about the marginal distributions chosen, however, the IFM estimator is superior to the CML method and it is extremely close to the EML estimator in terms of efficiency. We also gained some insight into the performance of different model selection criteria. The Akaike information criterion should be considered first when looking for the copula that most likely can capture the true dependence present in the data. The tests based on the conditional distribution function performed reasonable only for large samples and strong dependence. All three tests considered perform well in terms of size, but only the Jarque-Bera test, which had not been suggested in the literature for testing copula models until now, showed sufficient power to be recommended for empirical applications.

We used the techniques introduced to model the joint distribution of Latin American exchange rate returns against the Euro by fitting a copula to the standardized residuals of an ARMA-GARCH model. We found evidence of asymmetric dependence, both excess upper and lower tail dependence, as well as symmetric joint movements in the data we analyzed.

We can conclude that copula models are, and will be, a very useful tool in econometrics for simulation, density forecasting and for analyzing nonlinear dependence. The simulation algorithms offer themselves as a tool for realistically simulating asset returns, which may be used for pricing exotic options or credit risk derivatives by simulation methods. The ability of copulas to model the 
complete joint distribution of a variable of interest turns out to be useful when analyzing risk for a set of variables. A classical example is the Value-at-Risk of a portfolio using copula based dependence measure instead of Pearson's correlation coefficient. More copula based models in finance and risk management have arisen and can be expected to arise in the future. Furthermore, copulas can be used to model nonlinear dependencies between any type of economic variables. In particular the topic of time-varying copulas still poses many challenges for further research, and some issues will be dealt with in this thesis. Even though applications outside financial econometrics are rare, one can expect the development of such models in the near future. Additional theoretic research needs to be conducted regarding model selection tests and the modeling of higher dimensional copulas, but once they become a more common tool, higher dimensional copulas will most probably be used in many empirical applications. 


\section{Testing for asymmetric dependence ${ }^{1}$}

\subsection{Introduction}

Asymmetries in correlation of financial assets can have important implications for portfolio selection, hedging and pricing of options with multiple underlying assets. Several studies such as King and Wadhwani (1990), Longin and Solnik (1995) or Ramchand and Susmel (1998) have found that correlations increase during volatile periods. Such findings are supported by factor models for asset pricing, which imply that an increase in the volatility of the factors leads to higher correlations between different stocks or stock market indices. However, more recently Longin and Solnik (2001) noted that a "bear market, not volatility per se is the driving force in increasing correlation". Cappiello, Gérard, and Manganelli (2005) and Candelon and Manner (2007) even found that high volatility may decrease dependence between financial markets. Ang and Chen (2002) propose a test whether a given model can explain the asymmetric correlation and find evidence of asymmetries in stock returns. Based on this work Hong et al. (2007) provide a model free test for the null hypothesis of symmetric correlation, provide the asymptotic distribution of their test statistic and demonstrate the economic significance of taking into account asymmetries in investment decisions. Another study focusing on asymmetric dependence structures is Cappiello, Engle, and Sheppard (2007) who extended the well known DCC model for correlations and find evidence of asymmetries in conditional correlations.

A tool that has been frequently used to model asymmetric dependencies are copulas, which are that part of a multivariate distribution function that fully describes the dependence between the variables of interest (see Nelsen (2006) for an introduction and e.g. Patton (2006b), for an application). An important reason for using copulas is that many families allow for dependence in the extreme tails, a property known as tail dependence. Failing to capture dependence in the tails of the distribution may lead an underestimation of risks associated with crisis events. Although many authors have noted that certain copulas imply asymmetric dependence this has not been formalized

\footnotetext{
${ }^{1}$ This chapter is based on Manner (2010).
} 
and tested for in the copula context. Furthermore, usually only asymmetry in tail dependence is considered, but asymmetries at finite levels are not properly studied. In this chapter we try to bridge this gap. Instead of using linear correlation as our canonical measure of dependence we use the more robust Kendall's tau that has a number of desirable properties such as being invariant under monotone transformations of the underlying variables, see Joe (1997). Our first contribution is to define a conditional version of Kendall's tau and we provide formulas that can be used to evaluate the conditional Kendall's tau for a given copula. This allows us to assess the degree of asymmetry for two well known and frequently used families of copulas, namely the Gumbel and the Clayton (or Cook-Johnson) families. Based on these two copulas we construct two simple likelihood ratio tests for the null hypothesis of symmetric dependence. In a Monte Carlo experiment we compare these tests to the one proposed by Hong et al. (2007) and we find that these tests are clearly superior in terms of power, especially for small samples. Next, we suggest three simple data generating processes that can cause asymmetric dependence. The first two are returns generated by bear and bull markets with different mean returns, variances and correlations over the regimes, and data coming from a nonlinear VAR's, where ignoring the nonlinearity causes asymmetric dependence of the residuals. The third case makes use of the fact that copulas cannot only be used to model contemporaneous dependence of two (or more) variables, but that the class of Copula Markov Models, introduced by Joe (1997) and studied by Bouyé et al. (2001) and Chen and Fan (2006b), can be used to model dependence between a variable and its own lags. We show that data generated from a self exciting smooth transition autoregression (SETAR) can cause asymmetric dependence through time. These three cases are considered in a Monte Carlo study. From these examples it follows that finding asymmetric dependence does not necessarily mean that models allowing for asymmetric tail dependence must be used, as is quite often the case when modeling asset returns. Asymmetric dependence may simply suggest that linear models are not entirely appropriate for the data at hand and that care must be taken to account for nonlinearities.

In our empirical application we test for asymmetric dependencies in international stock market returns and reject the null of symmetric dependence for a majority of cases. We apply also the technique to quarterly returns of US GNP and unemployment data, that have been modeled with a threshold VAR by Altissimo and Violante (2001). In the autoregressive dynamics we find evidence of asymmetric dependence for unemployment, but not for GNP. Next, the data are fit to both a linear VAR and the threshold VAR by Altissimo and Violante (2001) and we run our tests on the residuals. The residuals of the linear VAR show asymmetries, whereas those of the nonlinear model are characterized by 
symmetric dependence.

The rest of the chapter is structured as follows. In Section 3.2 the concept of asymmetric dependence is introduced, the tests by Ang and Chen (2002) and Hong et al. (2007) are reviewed and we propose our alternative tests. Section 3.3 provides examples of data generating processes that can cause asymmetric dependence and these examples are analyzed. In Section 3.4 an extensive Monte Carlo study is provided that shows the behavior of the tests under different scenarios. In Section 3.5 an empirical application on international stock market returns and US macroeconomic data is provided and Section 3.6 concludes.

\subsection{Testing for asymmetric dependence}

We introduce the concept of exceedance correlation and we propose a conditional version of Kendall's tau in this section. Based on this we study the dependence properties of two well known families of copulas and we propose two tests for the null hypothesis of symmetric dependence using these copulas.

\subsubsection{Asymmetric dependence}

Let $X_{t}$ and $Y_{t}$, for $t=1, \ldots, T$, be two stochastic processes (e.g. stock returns) that are assumed to be $\beta$-mixing as in Chen and Fan (2006b) and Chen and Fan (2006a) and consider the exceedance correlation between the two as studied by Longin and Solnik (2001), Ang and Chen (2002) and Hong et al. (2007). Assume w.l.g. that $X_{t}$ and $Y_{t}$ have been standardized to have zero mean and unit variance. The exceedance correlation at level $c$ is defined as

$$
\begin{aligned}
& \rho^{+}(c)=\operatorname{corr}\left(X_{t}, Y_{t} \mid X_{t}>c, Y_{t}>c\right) \\
& \rho^{-}(c)=\operatorname{corr}\left(X_{t}, Y_{t} \mid X_{t}<-c, Y_{t}<-c\right) .
\end{aligned}
$$

The null hypothesis of symmetric correlation then is

$$
H_{0}: \quad \rho^{+}(c)=\rho^{-}(c), \quad \forall c \geqslant 0
$$

against

$$
H_{1}: \quad \rho^{+}(c) \neq \rho^{-}(c), \quad \text { for } \text { some } \quad c \geqslant 0 .
$$


Denote the estimates of the exceedance correlation by $\hat{\rho}^{+}(c)$ and $\hat{\rho}^{-}(c)$ and let $\rho(c, \phi)$ be the exceedance correlation implied by a given model with parameter $\phi$. Then the test statistic proposed by Ang and Chen (2002) is given by ${ }^{2}$

$$
H^{2}=\sum_{i=1}^{m} w\left(c_{i}\right)\left(\rho\left(c_{i}, \phi\right)-\hat{\rho}^{+}\left(c_{i}\right)\right)^{2}
$$

where $c_{1}, \ldots, c_{m}$ are $m$ exceedance levels and $w\left(c_{i}\right)$ is a weight. $\hat{\rho}^{+}(c)$ may be replaced by $\hat{\rho}^{-}(c)$ in the formula. Hong et al. (2007) derive the asymptotic distribution of $H^{2}$, which is a mixture of $m$ independent chi-squared random variables with weights equal to the eigenvalues of the weighted covariance matrix of $\hat{\rho}$. This test can be used to test whether a given model can explain the empirical exceedance correlation.

The test proposed by Hong et al. (2007) is model free and can be used to test the null hypothesis given in (3.3). For

$$
\hat{\rho}^{+}-\hat{\rho}^{-}=\left[\hat{\rho}^{+}\left(c_{1}\right)-\hat{\rho}^{-}\left(c_{1}\right), \ldots, \hat{\rho}^{+}\left(c_{m}\right)-\hat{\rho}^{-}\left(c_{m}\right)\right]^{\prime}
$$

and $T$ the sample size, their test statistic is

$$
J_{\rho}=T\left(\hat{\rho}^{+}-\hat{\rho}^{-}\right)^{\prime} \hat{\Omega}^{-1}\left(\hat{\rho}^{+}-\hat{\rho}^{-}\right),
$$

where $\hat{\Omega}$ is a heteroscedasticity and autocorrelation (HAC) robust covariance estimator like the one proposed by Newey and West (1994). Under the assumptions of fourth order stationarity, still allowing for volatility clustering, and a strong mixing condition ruling out long memory of the processes $X_{t}$ and $Y_{t}$ this statistic is shown to asymptotically follow a chi-square distribution with $m$ degrees of freedom. Additional assumptions needed are a finite and nonsingular covariance matrix $\Omega$ and some standard requirements on the Kernel and (data driven) bandwidth used for covariance estimation. Both approaches measure dependence through the correlation coefficient. As a measure of dependence, however, linear correlation is only appropriate when the data follows an elliptical distribution. For drawbacks of using linear correlation for modeling dependence see Embrechts et al. (2002).

As an alternative we suggest using the concept of concordance for measuring dependence. Consider two pairs of observations $\left(x_{i}, y_{i}\right)$ and $\left(x_{j}, y_{j}\right)$ from $\left(X_{t}, Y_{t}\right)$. We call these pairs of observations concordant if $\left(x_{i}-x_{j}\right)\left(y_{i}-y_{j}\right)>0$ and discordant if $\left(x_{i}-x_{j}\right)\left(y_{i}-y_{j}\right)<0$. Hence, two random

\footnotetext{
${ }^{2}$ Strictly speaking $H^{2}$ cannot be called a statistic, since it depends on the unknown parameter $\phi$, but it can be calculated once an estimate $\hat{\phi}$ has been obtained.
} 
variables are said to be concordant, when large values of one random variable tend to be associated with large values of the other, and similarly small values tend to be associated with each other.

Using the concept of concordance we are now able to introduce a measure of association known as Kendall's tau. Its sample version is defined as the fraction of concordant pairs of observations in the sample minus the fraction of discordant pairs of observations. The population version of Kendall's tau is defined as the difference between the probability of concordance and the probability of discordance.

$$
\tau=\tau\left(X_{t}, Y_{t}\right)=P\left[\left(X_{i}-X_{j}\right)\left(Y_{i}-Y_{j}\right)>0\right]-P\left[\left(X_{i}-X_{j}\right)\left(Y_{i}-Y_{j}\right)<0\right] \text { for } i \neq j .
$$

Since Kendall's tau is only based on the ranks of the observations it is, unlike the correlation coefficient, invariant under monotone transformations of $X_{t}$ and $Y_{t}$. Therefore, consider the random variables $U_{t}=F\left(X_{t}\right)$ and $V_{t}=G\left(Y_{t}\right)$, where $F$ and $G$ are the (unconditional) marginal distributions of $X_{t}$ and $Y_{t}$, respectively, which we assume to exist. We define the analogue to exceedance correlation, which we call exceedance Kendall's tau, as

$$
\begin{aligned}
& \tau^{+}(c)=\tau\left(U_{t}, V_{t} \mid U_{t}>c, V_{t}>c\right) \\
& \tau^{-}(c)=\tau\left(U_{t}, V_{t} \mid U_{t}<1-c, V_{t}<1-c\right) .
\end{aligned}
$$

In this chapter, whenever we speak of $\tau^{+}$and $\tau^{-}$without specifying an exceedance level we mean $\tau(U, V \mid U>0.5, V>0.5)$ and $\tau(U, V \mid U<0.5, V<0.5)$. Thus they are the exceedance Kendall's tau for the exceedance level being the median of the distributions of $X$ and $Y$. The hypothesis of symmetric dependence we are interested in then becomes

$$
H_{0}: \quad \tau^{+}(c)=\tau^{-}(c), \quad \forall c \geqslant 0
$$

against

$$
H_{1}: \quad \tau^{+}(c) \neq \tau^{-}(c), \text { for some } c \geqslant 0 \text {. }
$$

In the next section we introduce a methods for testing the equality of the $\tau^{+}(c)$ and $\tau^{-}(c)$ for all $c$.

\subsubsection{A test based on mixtures of copulas}

As before, let $F$ be the marginal distribution function of $X_{t}, G$ be the marginal distribution function of $Y_{t}$, and let $H$ be the joint distribution function of the pair of random variables $\left(X_{t}, Y_{t}\right)$. Then by 
the copula representation of multivariate distribution functions there exists a copula $C$ such that

$$
H(x, y)=C(F(x), G(y)), \forall(x, y) \in \overline{\mathbf{R}} \times \overline{\mathbf{R}},
$$

where $\overline{\mathbf{R}}$ denotes the extended real line. If $F$ and $G$ are continuous then $C$ is unique. Conversely if we have distribution functions $F$ and $G$ and a copula $C$, then $H$ is a bivariate distribution function. Recalling the probability integral transform for continuous distributions, which states that the random variable $U_{t}=F\left(X_{t}\right)$ has a $U(0,1)$ distribution regardless of the original distribution $F$, it becomes clear that a copula is no more than a multivariate distribution function with uniform marginals. It captures all the contemporaneous dependence between the random variables of interest, as all the dynamics of the marginal distributions are captured by $F$ and $G$ for $X_{t}$ and $Y_{t}$, respectively.

Kendall's tau between $X_{t}$ and $Y_{t}$ is determined by the underlying copula, which results from Theorem 5.1.3 in Nelsen (2006). For $\left(U_{t}, V_{t}\right)=\left(F\left(X_{t}\right), G\left(Y_{t}\right)\right) \sim C_{\theta}(u, v)$ it boils down to the statement that

$$
\tau_{C}=4 E\left(C_{\theta}\left(U_{t}, V_{t}\right)\right)-1
$$

where $E$ denotes the unconditional expectation. For many copulas this expression can be solved to express $\tau$ in terms of the copula parameters. One parameter copulas can even be estimated by inverting the relationship between $\tau$ and the copula parameter $\theta$, resulting in a moment estimator. For a display of this method see van den Goorbergh, Genest, and Werker (2005).

The conditional version of Kendall's tau can also be expressed in terms of the underlying copula. Proposition 3.1. Let the joint distribution of $U_{t}$ and $V_{t}$ be $C_{\theta}(u, v)$. Then the population version of $\tau^{-}$defined in (3.10) for $c=0.5$ is determined by the underlying copula through

$$
\tau_{C}^{-}=\frac{E\left[4 C_{\theta}\left(U_{t}, V_{t}\right)-2 C_{\theta}\left(U_{t}, 0.5\right)-2 C_{\theta}\left(0.5, V_{t}\right) \mid U_{t}<0.5, V_{t}<0.5\right]}{C_{\theta}(0.5,0.5)}+1 .
$$

The population version of $\tau^{+}$defined in (3.9) for $c=0.5$ is given by

$$
\tau_{C}^{+}=\frac{E\left[4 C_{\theta}\left(U_{t}, V_{t}\right)-2 C_{\theta}\left(U_{t}, 0.5\right)-2 C_{\theta}\left(0.5, V_{t}\right)-1 \mid U_{t}>0.5, V_{t}>0.5\right]}{C_{\theta}(0.5,0.5)}+1
$$

A proof is given is given in the appendix. It is straightforward to derive formulas for exceedance levels different from 0.5. The conditional expectations can in general not be evaluated analytically. However, since it is computationally easy to simulate from most parametric classes of copulas one 
Figure 3.1: Contour plots and simulated data from the Gumbel and Clayton Copula
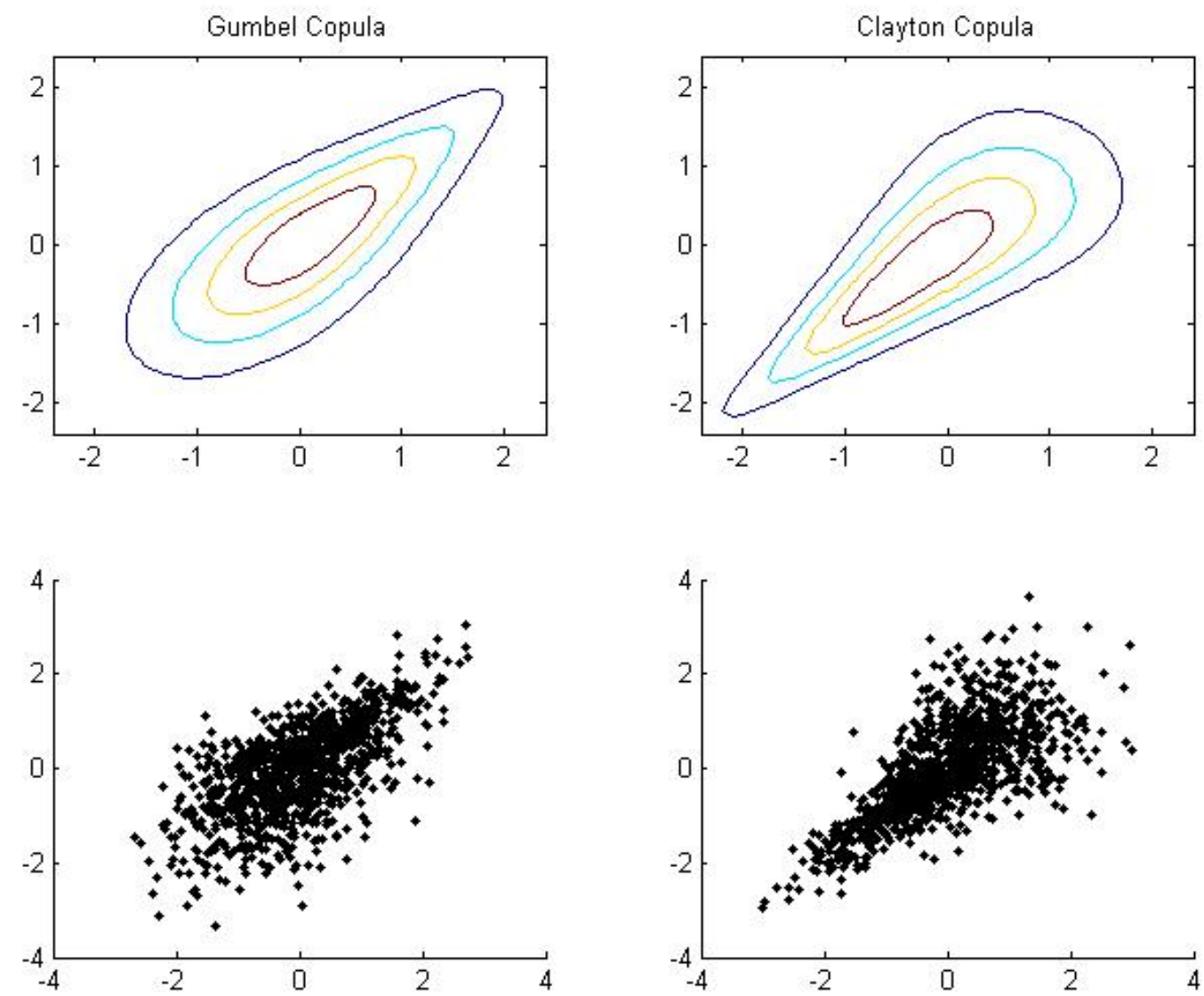

can evaluate these expectations numerically at any desired precision.

Two copulas that have received a lot of attention in the literature are the Gumbel and the Clayton copulas. Their distribution functions are given by

$$
C_{\theta}^{\text {Gumbel }}(u, v)=\exp \left(-\left[(-\ln (u))^{\theta}+(-\ln (v))^{\theta}\right]^{1 / \theta}\right)
$$

and

$$
C_{\theta}^{\text {Clayton }}(u, v)=\max \left[\left(u^{-\theta}+v^{-\theta}-1\right)^{\frac{-1}{\theta}}, 0\right] .
$$

They belong to the class of Archimedean copulas and they are known to imply asymmetric dependence. In particular, they are characterized by tail dependence in one tail, and tail independence in the other. Contour plots and simulated data of the two copulas with standard normal margins and 
Figure 3.2: $\tau^{+}(0.5)$ and $\tau^{-}(0.5)$ for the Gumbel and Clayton copulas
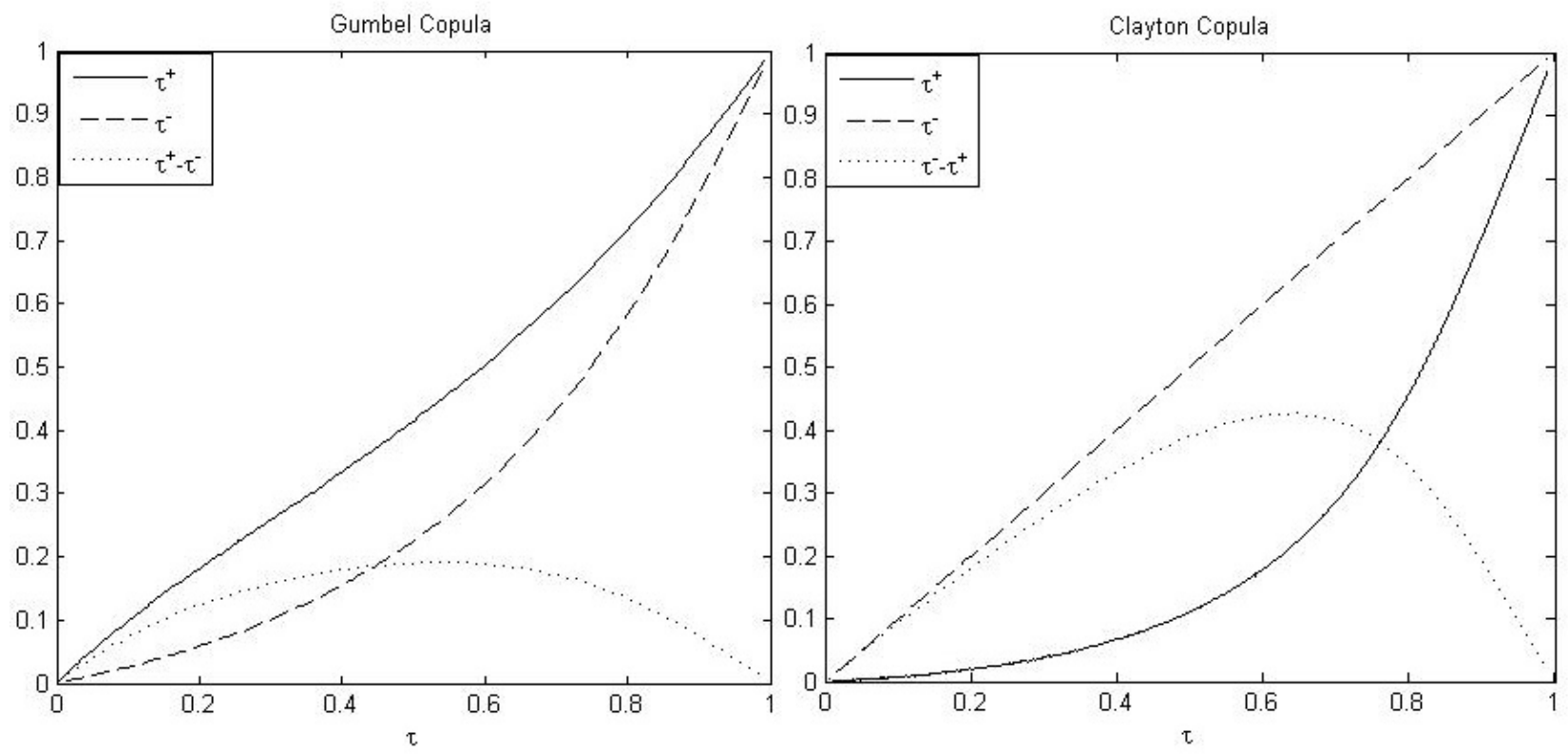

parameters corresponding to Kendall's tau equal to 0.5 are provided in Figure 3.1. Using the results from Proposition 3.1 we show that these two copulas imply asymmetric dependence. We evaluated the conditional expectations using 1000000 simulated data points and we let the parameters of the copulas vary corresponding to an overall Kendall's tau ranging from 0.01 to 0.99 . In Figure $3.2 \tau^{+}$ and $\tau^{-}$for $c=0.5$ are plotted along with their difference. One can see that both copulas imply asymmetric dependence, but that the asymmetry is larger for the Clayton copula. Especially for lower overall dependence $\tau^{+}$is extremely small for the Clayton copula, which explains why often it does not fit financial data too well, even though there is evidence of higher dependence for losses than for gains.

Given a copula $C(u, v)$ the corresponding survival, or rotated, copula is given by $C(1-u, 1-$ $v)+u+v-1$. Using the fact that the convex combination of two copulas is again a copula two very flexible copulas are the Gumbel mixture (GM) and the Clayton mixture (CM) copulas given by

$$
C_{\alpha, \theta_{1}, \theta_{2}}^{G M}(u, v)=\alpha \cdot C_{\theta_{1}}^{G u m b e l}(u, v)+(1-\alpha) \cdot\left(C_{\theta_{2}}^{G u m b e l}(1-u, 1-v)+u+v-1\right)
$$

and

$$
C_{\alpha, \theta_{1}, \theta_{2}}^{C M}(u, v)=\alpha \cdot C_{\theta_{1}}^{\text {Clayton }}(u, v)+(1-\alpha) \cdot\left(C_{\theta_{2}}^{\text {Clayton }}(1-u, 1-v)+u+v-1\right) .
$$


These Copulas nests symmetry when, jointly, $\alpha$ is equal to 0.5 and $\theta_{1}=\theta_{2}$, which follows from Theorem 2.7.3 in Nelsen (2006).

Denote the densities of the models in either (3.19) or (3.20) by $c(u, v ; \theta)$, with $\theta=\left(\alpha, \theta_{1}, \theta_{2}\right)$. Then the maximum likelihood estimator $\hat{\theta}$ is

$$
\hat{\theta}=\operatorname{argmax} \sum_{t=1}^{T} \log c\left(U_{t}, V_{t} ; \theta\right),
$$

whereas $\hat{\theta}_{\text {res }}$ is the maximum likelihood estimator under the restriction that $\alpha=0.5$ and $\theta_{1}=\theta_{2}$. Then the null hypothesis of symmetric dependence at all possible exceedance levels given in (3.11) can be tested with the likelihood ratio statistic

$$
L R=\sum_{t=1}^{T} \log \frac{c\left(U_{t}, V_{t} ; \hat{\theta}\right)}{c\left(U_{t}, V_{t} ; \hat{\theta}_{\text {res }}\right)} .
$$

Note that unless the data are assumed to be i.i.d. and the model is correctly specified this is in fact a pseudo likelihood ratio statistic. The case of a misspecified model is discussed in detail below, but we must make the assumption of i.i.d. data. See remark 1 below on how this can be handled in practice. The distribution of the $L R$ statistic depends on whether the GM or CM copulas are the true ones or not. Under a correctly specified model and standard regularity conditions it is well known that $2 L R \sim \chi_{2}^{2}$.

However, if the model is misspecified the situation is a little more difficult. Let $\ell(u, v, \theta)=$ $\log c(u, v ; \theta)$ be the $\log$-likelihood function, $\ell_{\theta}(u, v ; \theta)=\frac{\partial}{\partial \theta} \ell(u, v, \theta)$ the score, and $\ell_{\theta \theta}(u, v ; \theta)=$ $\frac{\partial^{2}}{\partial \theta \partial \theta^{\prime}} \ell(u, v, \theta)$ the Hessian. Further let $B=E^{0}\left[\ell_{\theta \theta}\left(U_{t}, V_{t} ; \theta^{*}\right)\right], \Sigma=E^{0}\left[\ell_{\theta}\left(U_{t}, V_{t} ; \theta\right) \ell_{\theta}\left(U_{t}, V_{t} ; \theta\right)^{\prime}\right]$, and $\Sigma_{12}=\operatorname{cov}^{0}\left(\ell_{1, \theta}\left(U_{t}, V_{t} ; \theta_{1}^{*}\right), \ell_{2, \theta}\left(U_{t}, V_{t} ; \theta_{2}^{*}\right)\right)$ the covariance of the scores of two models, where $\theta^{*}$ is the pseudo true value of the parameter and $E^{0}$ means that the expectations are taken under the true model. Then under general conditions Vuong (1989) showed for a fully parametric model that $2 L R \sim M\left(\cdot, \lambda^{*}\right)$, where $M\left(\cdot, \lambda^{*}\right)$ is the distribution of a weighted sum of independent $\chi_{1}^{2}$ random variables with weights $\lambda^{*}=\left(\lambda_{1}^{*}, \ldots, \lambda_{4}^{*}\right)^{\prime}$ equal to the eigenvalues of the $4 \times 4$ matrix

$$
W=\left(\begin{array}{cc}
\Sigma_{u r} B_{u r}^{-1} & -\Sigma_{12}^{\prime} B_{\text {res }}^{-1} \\
\Sigma_{12} B_{u r}^{-1} & -\Sigma_{r e s} B_{\text {res }}^{-1}
\end{array}\right),
$$

where the subscripts denote the matrices corresponding to unrestricted and restricted models. For inference the sample analogues of the matrices must be computed. 
The situation is further complicated when the margins are estimated by the empirical CDF as studied in Chen and Fan (2005) and Chen and Fan (2006a). When calculating the matrices $\Sigma$ and $\Sigma_{12}$, the score $\ell_{\theta}(u, v ; \theta)$ must be replaced by $\ell_{\theta}(u, v ; \theta)+W_{u}(u, v ; \theta)+W_{v}(u, v ; \theta)$, where $W_{u}=E^{0}\left[\ell_{\theta u}\left(U_{t}, V_{t} ; \theta\right)\left\{I\left(U_{t} \leqslant U_{s}\right)-U_{s}\right\} \mid U_{t}\right]$, with $\ell_{\theta u}(u, v ; \theta)=\frac{\partial^{2}}{\partial \theta \partial u} \ell(u, v, \theta)$ and $I$ the indicator function. The term $W_{v}$ is defined similarly. These terms are introduced by the need to estimate the marginal distributions and they disappear in the case of known marginals. Critical values can be obtained by calculating the sample analogues of $W_{u}$ and $W_{v}$. Alternatively, Chen and Fan (2005) suggest a bootstrap algorithm to approximate this asymptotic distribution and they show its validity.

Remark 1: In order to estimate one of the suggested copula models the variables of interest must be $U(0,1)$ distributed. Since we are not interested in the marginal distributions we can simply achieve that by using the empirical distribution function, which is given by

$$
\hat{F}(y)=\frac{1}{T} \sum_{t=1}^{T} \mathbf{1}_{\left\{Y_{t} \leqslant y\right\}} .
$$

This means that we are using the semi-parametric estimator studied in detail by Genest et al. (1995) for the i.i.d. case and by Chen and Fan (2006a) for time series. Chen and Fan (2006b) show that the empirical CDF consistently estimates the marginal distributions under the assumption of $\beta$-mixing. However, in practice parametric models such as AR-GARCH specifications are estimated in a first step to remove mean and variance dynamics, and the empirical CDF is applied to the standardized residuals, to achieve i.i.d.'ness of the transformed variables, which is required for the asymptotic results to hold.

Remark 2: The assumptions under which the above results hold are can be found in Chen and Fan (2006a) and are assumed to hold. However, care must be taken on the assumption the pseudo true values of the parameters lie in the interior of the parameters space. This may be violated when the mixing parameter $\alpha$ in (3.19) or (3.20) is close to zero or one, which may happen when the data show tail dependence only in one tail. In that case the critical values cannot be relied on. On the other hand, a mixing parameter close to the boundary itself is a strong indication of asymmetric dependence.

Remark 3: In practice it is rather difficult to know whether one of the copulas we propose for testing the null hypothesis of symmetric dependence is indeed the true one and hence which critical values need to be used. A possible solution is to perform a Goodness-of-fit test on the unrestricted GM 
and CM copulas and only use the standard critical values when the null of correct specification is not rejected. We recommend to test this null by performing an Anderson-Darling test for uniformity on the Rosenblatt transformation $Z_{t}=C\left(U_{t} \mid V_{t} ; \hat{\theta}\right)$ as suggested in e.g. Dobric and Schmid (2007). Nevertheless, it is always safe to use the critical values based on the mixture $\chi^{2}$ distribution, because this distribution reduces to the standard $\chi_{2}^{2}$ distribution under a correctly specified model, see Vuong (1989).

From now on we denote the test based on the Gumbel mixture by GM-test and the one based on the Clayton mixture copula by CM-test. The use of these two copulas is motivated by the fact that the Clayton copula can capture very strong asymmetries. The Gumbel copula (or its survival version), on the other hand, tends to fit financial and economic data quite well in empirical applications.

\subsubsection{Local power}

In this section we focus on the simpler case of a correctly specified model, i.e. assuming data is coming from model (3.19) or (3.20). There are two reasons why we believe that our tests have higher power than the ones by Ang and Chen (2002) and Hong et al. (2007). First, as mentioned already Kendall's tau is a more robust measure of dependence and it is more appropriate when the data is not normally distributed, which is likely to be the case for most financial data. Second, when computing the two mentioned tests one may have to discard a large fraction of the data, in particular when the overall dependence is rather weak. This is not the case for the copula based test and thus its efficient use of the data available should lead to better size and power properties. We investigate this argument by studying the local power properties of the GM and CM tests in comparison with the $J$ - test by Hong et al. (2007). Consider the GM and CM models given in (3.19) and (3.20) with $\theta_{1}=\theta_{2}=2$ corresponding to an overall Kendall's tau of 0.5. When $\alpha=0.5$ the null hypothesis holds and $2 L R$ is $\chi_{2}^{2}$ distributed. Under the sequence of local alternatives $\alpha=0.5+h / \sqrt{T}$, the statistic $2 L R$ follows a noncentral $\chi^{2}$ distribution with non-centrality parameter $\delta=h^{2} \cdot \sqrt{\Sigma_{\alpha}}$, where $\Sigma_{\alpha}$ is the entry of the Fisher information matrix defined above corresponding the mixing parameter $\alpha$, see van der Vaart (1998). We approximate $\Sigma_{\alpha}$ numerically. In principle the distribution of the $J$-test under local alternatives is known as well, since it is a Wald test. However, the true exceedance correlations and their covariance under the GM and CM models are unknown and thus we must approximate these numbers by Monte Carlo simulation. We consider the exceedance quantiles of $\mathrm{c}=0.5,0.7,0.9$. The local power functions in Figure 3.3 show that for the GM test the local power curve is flatter than 
Figure 3.3: Local power functions against $\sqrt{T}$ alternatives
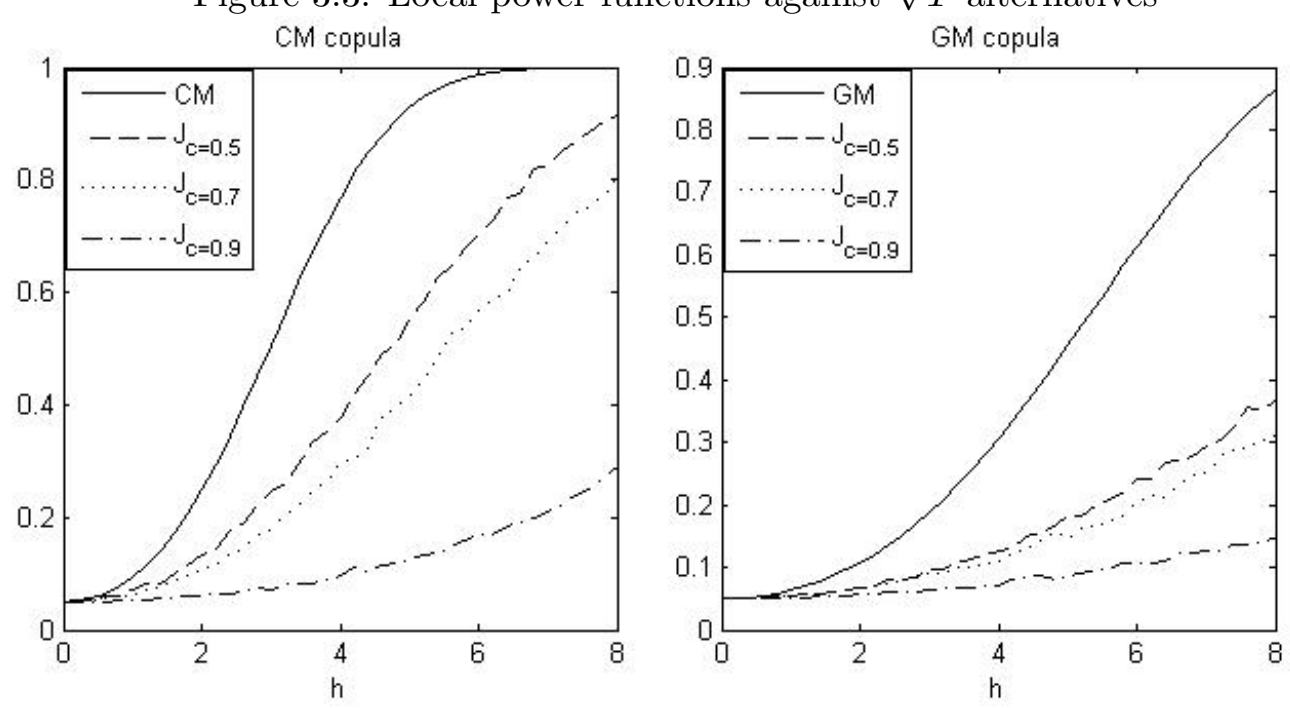

that of the CM test for the same sequence $h$. This is a result of the fact that the Gumbel copula implies less asymmetry than the Clayton copula for the same overall dependence as seen in Figure 3.2. This can also be seen by comparing the local power curves of the $J$-test across the two figures. Furthermore, the copula based tests have much higher power than the $J$-test and the $J$-test loses power as $c$ increases. This is not surprising as one has $T$ observations available to estimate each parameter for the GM and CM tests, but only $P\left[U_{t}>c, V_{t}>c\right] \cdot T$ observations for the $J-$ test at exceedance level $c$. In practice this means that even though one could in principle allow $c$ to increase towards 1 with the sample size ${ }^{3}$ it is optimal to chose $c=0.5$ or to chose multiple exceedance levels including the optimal choice.

In the rest of the chapter we do not make the assumption of a correctly specified model anymore, but instead use the mixture of $\chi_{1}^{2}$ distributions for inference.

\subsection{How asymmetry is generated}

In this section we consider a few data generating processes (DGP) that imply asymmetric dependence even though the innovations were generated by a multivariate normal distribution. In the next section we perform Monte Carlo simulations to study these examples.

\footnotetext{
${ }^{3}$ In fact, $c$ could increase at rate $T$, e.g. $c=1-k / T$ for some positive $k$, for data that has positive tail dependence, but at a slower rate for data coming from a distribution with independent tails. In the latter case the rate depends on the speed of decline of the tail probabilities, see e.g. Ledford and Tawn (1996) for some examples.
} 


\section{Example 1: Bull and Bear Markets}

Let $R_{1 t}$ and $R_{2 t}$ be the returns in stock market 1 and 2 in period $t$. It is a stylized fact that stock markets have low volatility and correlation in periods of high returns (bull markets) and that volatility and correlation tend to increase when market have low (mostly negative) returns (bear markets). This corresponds to regime switching models for international equity markets such as the one considered in Ramchand and Susmel (1998). Consider the case that returns are generated by the following DGP in state $s=h, l$, where $h$ stands for bull state and $l$ for the bear state:

$$
\begin{gathered}
R_{1 t s}=\mu_{1 s}+\epsilon_{1 t s} \\
R_{2 t s}=\mu_{2 s}+\epsilon_{2 t s},
\end{gathered}
$$

where

$$
\left(\begin{array}{c}
\epsilon_{1 t s} \\
\epsilon_{2 t s}
\end{array}\right) \sim N\left[\left(\begin{array}{l}
0 \\
0
\end{array}\right),\left(\begin{array}{cc}
\sigma_{1 s}^{2} & \rho_{s} \sigma_{1 s} \sigma_{2 s} \\
\rho_{s} \sigma_{1 s} \sigma_{2 s} & \sigma_{2 s}^{2}
\end{array}\right)\right]
$$

If one ignores the state dependence of the distributions of $R_{1}$ and $R_{2}$ they will exhibit asymmetric dependence. As an example consider the simplified case that all standard deviations are equal to the mean return in the high state $\sigma_{1 h}=\sigma_{1 l}=\sigma_{2 h}=\sigma_{2 l}=\mu_{1 h}=\mu_{2 h}$, the mean returns in the bear state are minus the mean returns in the bull state $\mu_{1 l}=\mu_{2 l}=-\mu_{h}$, and the correlations are $\rho_{h}=0.3$ and $\rho_{l}=0.7$. Then the probabilities of observing joint exceedances of zero returns are $P\left(R_{1 l}>0, R_{2 l}>0\right)=0.084, P\left(R_{1 l}<0, R_{2 l}<0\right)=0.7667, P\left(R_{1 h}>0, R_{2 h}>0\right)=0.7281$, and $P\left(R_{1 h}<0, R_{2 h}<0\right)=0.0455$. Therefore the correlation between positive returns is dominated by the lower correlation during the bull state, whereas the correlation for negative returns is dominated by the higher correlation during the bear state, and together this implies asymmetric dependence. If additionally we assume that both state are equally likely we can calculate $\tau^{+}$and $\tau^{-}$for this setting using the formulas in Proposition 3.1. The corresponding copula is a mixture of two Gaussian copulas with $\rho_{1}=0.3$ and $\rho_{2}=0.7$, where the mixing parameter can be calculated using the exceedance probabilities calculated here. For $\tau^{+}$the weights are 0.9155 and 0.0845 and for calculating $\tau^{-}$they are 0.0691 and $0.9309 .{ }^{4}$ For $c=0.5 \tau^{-}$then is 0.2671 and $\tau^{+}$is 0.0997 .

\footnotetext{
${ }^{4}$ The weights are found such that the probabilities of exceedance for the mixing copula are equal to the ones found in the example. Using the CDF of the Gaussian copula at $(0.5,0.5)$ for $\rho$ equal to 0.3 and 0.7 this is straightforward.
} 


\section{Example 2: Nonlinear Multivariate Models}

A second set of DGP's that may lead to the observation of asymmetric dependence are nonlinear multivariate models. Let the random variable $X$ and $Y$ be generated as follows

$$
\begin{aligned}
& X_{t}=\delta I\left(\epsilon_{2 t}>c\right)+\epsilon_{1 t} \\
& Y_{t}=\delta I\left(\epsilon_{1 t}>c\right)+\epsilon_{2 t} .
\end{aligned}
$$

$\epsilon_{1 t}$ and $\epsilon_{2 t}$ are, possibly correlated, $N(0,1)$ variables. In general, dynamics should be allowed for and the model could be a nonlinear VAR, but for pedagogical purposes we stick to this simplified version for now. In the simulation study, however, we do allow for dynamics. This is similar to the model for stock market contagion considered by Pesaran and Pick (2007). Consider regressing the $X$ and $Y$ on an intercept and collecting the residuals, which then are tested for asymmetric dependence. The population version of the linearized model is

$$
\begin{aligned}
& X_{t}=\delta P\left(\epsilon_{2 t}>c\right)+\epsilon_{1 t}^{*} \\
& Y_{t}=\delta P\left(\epsilon_{1 t}>c\right)+\epsilon_{2 t}^{*} .
\end{aligned}
$$

Denote $P\left(\epsilon_{1 t}>c\right)=P\left(\epsilon_{2 t}>c\right) \equiv a$. Then the mean of the linearized model is $a \delta$ and the relation between the errors of the linearized model and the true errors is

$$
\begin{array}{r}
\epsilon_{1 t}^{*}=\epsilon_{1 t}+\delta I\left(\epsilon_{2 t}>c\right)-a \delta \\
\epsilon_{2 t}^{*}=\epsilon_{2 t}+\delta I\left(\epsilon_{1 t}>c\right)-a \delta .
\end{array}
$$

Due to the nonlinear nature of the DGP we cannot calculate the $\tau^{+}$and $\tau^{-}$directly. However, we can get an intuitive idea how asymmetry is generated for this DGP. To do so we must distinguish the cases where $c>a \delta$ and $c<a \delta$. For the second case the situation with $c=0$ is the most important one.

Case 1: $c>a \delta$

In this situation the probabilities that the errors of the linearized model jointly lie below and above 
zero can be shown to be

$$
\begin{aligned}
& P\left(\epsilon_{1 t}^{*}<0, \epsilon_{2 t}^{*}<0\right)=P\left(\epsilon_{1 t}<a \delta, \epsilon_{2 t}<a \delta\right) \\
& P\left(\epsilon_{1 t}^{*}>0, \epsilon_{2 t}^{*}>0\right)=P\left(\epsilon_{1 t}>a \delta, \epsilon_{2 t}>a \delta\right)+2 P\left(a-1<\epsilon_{1 t}<a \delta, \epsilon_{2 t}>c\right) .
\end{aligned}
$$

Clearly, the $\tau^{-}$is not affected by threshold effects, because all of the original errors that generate these observation jointly lie below the thresholds. The $\tau^{+}$on, the other hand, is affected, but it is a bit delicate to see in what way. The first part of $P\left(\epsilon_{1 t}^{*}>0, \epsilon_{2 t}^{*}>0\right)$ can be decomposed into those original errors that jointly exceed the threshold and those that do not. Once both errors exceed the threshold, the errors from the linearized model jump by the value $\delta$. A second pair of observations from the original errors for which only one variable exceeds the threshold is now more likely of being concordant with this first pair than before, since one of the components may have been larger than the corresponding one from the first pair. After the effect of the jump this may not be the case anymore.

It is unclear in what way concordance is affected for the terms in the second part of the probability. We expect the effect to be more or less neutral.

Case 2: $c<a \delta$

Now the probabilities that the residuals of the linearized model jointly lie below and above zero are

$$
\begin{aligned}
& P\left(\epsilon_{1 t}^{*}<0, \epsilon_{2 t}^{*}<0\right)=P\left(\epsilon_{1 t}<c, \epsilon_{2 t}<c\right) \\
& P\left(\epsilon_{1 t}^{*}>0, \epsilon_{2 t}^{*}>0\right)=P\left(\epsilon_{1 t}>c, \epsilon_{2 t}>c\right)+2 P\left(a-1<\epsilon_{1 t}<c, \epsilon_{2 t}>c\right) .
\end{aligned}
$$

The effect of the threshold will be constant when the original errors are both above the threshold and the effect described in case 1 cannot occur anymore. Thus we expect the asymmetry generated in this case to be rather small.

In Figure 3.4 we present a scatterplot of data generated from the this model with $\delta=0.7$ and correlation of 0.3 between the errors for $c=0$ and $c=0.5$. For a better exposition the variables have been mapped into $U(0,1)$ variables by the empirical probability integral transform. For $c=0.5$ the asymmetry is quite apparent and the data looks similar to observations generated by a Gumbel or a 
Figure 3.4: Simulated data for example 2
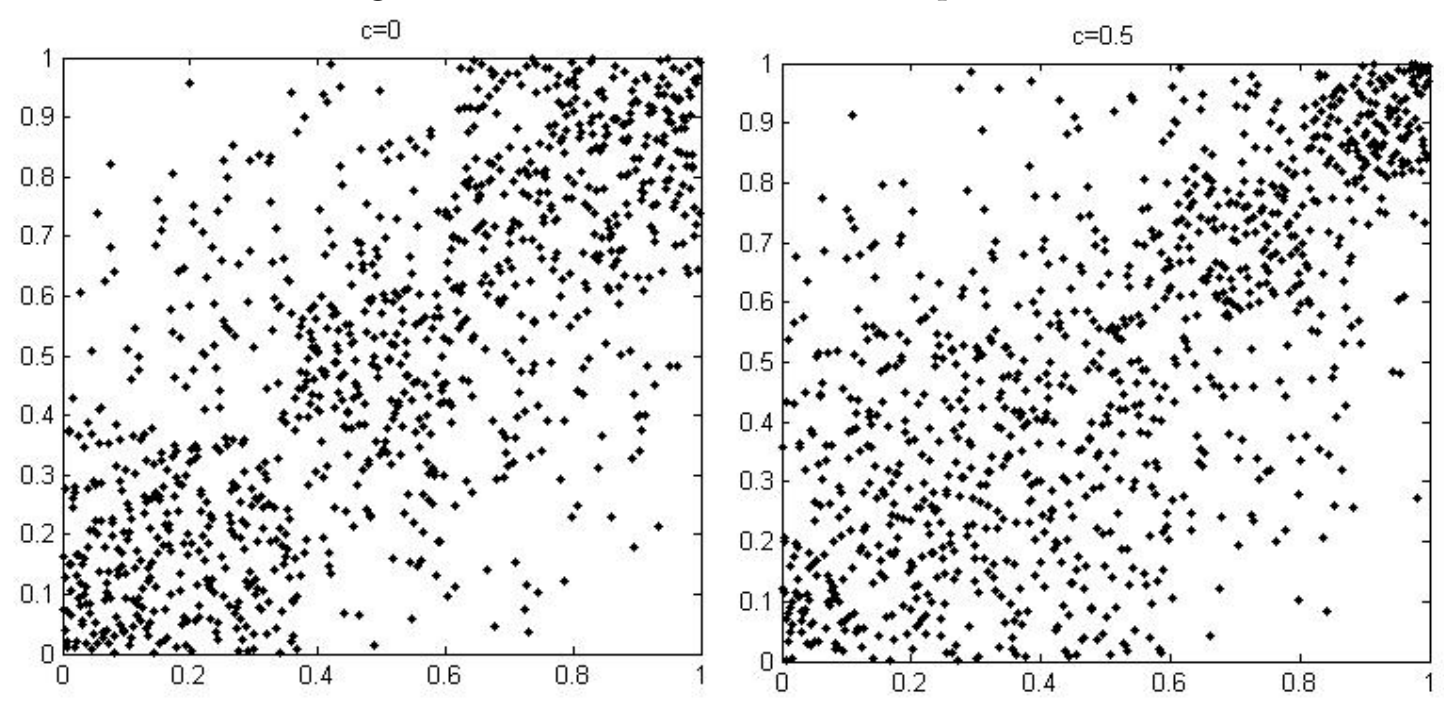

survival Clayton copula. In the plot for $c=0$ the dependence seems to be rather symmetric.

Finally, we evaluated the $\tau^{+}$and $\tau^{-}$by Monte Carlo simulation. For $c=0$ they are 0.2393 and 0.2379 , whereas for $c=0.5$ they turn out to be 0.3622 and 0.0896 , respectively. Thus the conclusions of our informal argument above are confirmed.

\section{Example 3: Nonlinear Autoregressions}

Copulas cannot only be used to model the contemporaneous dependence between i.i.d. random variables, but they may be used to model time dependence of a single variable. Instead of describing the dependence between $X_{t}$ and $Y_{t}$ one can fit a copula to $Y_{t}$ and $Y_{t-1}$, thus generalizing the normal $\mathrm{AR}(1)$ model for stationary variables and treating the random process $Y_{t}$ as a stationary first order Markov chain. More generally, a copula of dimension $p$ can be used to model autoregressive processes of order $p-1 .^{5}$ These models are called Copula Markov models. For details on these models see Joe (1997) or Bouyé et al. (2001). Chen and Fan (2006b) establish the validity of the semi-parametric estimation approach to this type of models when $Y_{t}$ is a stationary Markov chain of order 1 , namely they present conditions for processes generated by this type of models to be $\beta$-mixing and they establish consistency and asymptotic normality. Furthermore, they suggest an estimator for the asymptotic variance of the estimator. These results justify the validity of applying our tests for

\footnotetext{
${ }^{5}$ The bivariate margins of $(i, i+1)$ need to be restricted to be the same for all $i$.
} 
Figure 3.5: Difference between $\tau^{+}$and $\tau^{-}$for data generated by a SETAR model

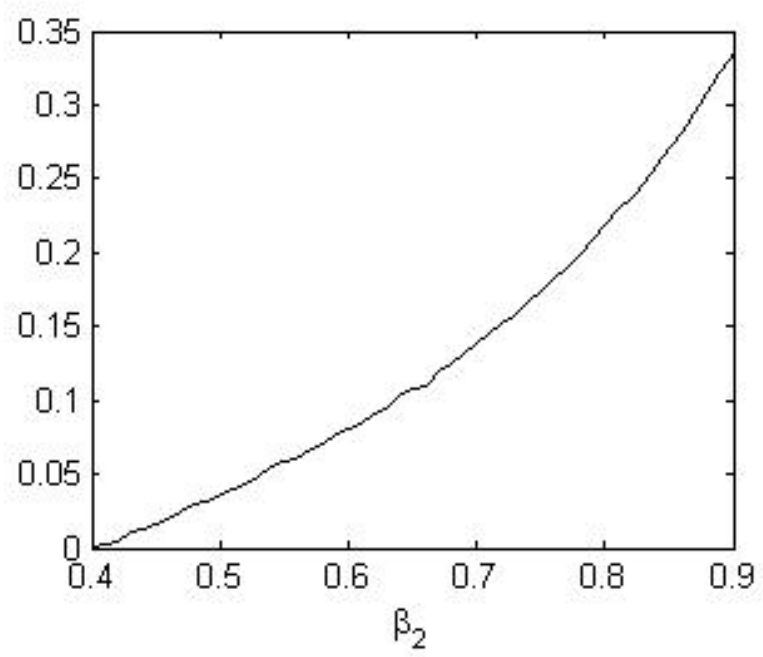

symmetric dependence on $Y_{t}$ and $Y_{t-1}$ whenever $Y_{t}$ can be treated as a stationary first order Markov chain and the asymptotic $\chi^{2}$ distribution of the likelihood ratio test statistic remains appropriate. In case the dynamics of a time series of interest are best described by a Gaussian copula and the margins are also normal, meaning linear dependence is appropriate, we are just replicating a linear AR model. However, in case the dependence is of an asymmetric type, a linear AR model may not be an appropriate model for the dynamics present in the data anymore. Conversely, data generated by a nonlinear autoregressive process is likely to be show asymmetric dependence. The example we consider here is the class of smooth transition autoregressions (STAR). For a survey on STAR models see van Dijk, Teräsvirta, and Franses (2002). Thus in the model of interest $Y_{t}$ is generated by

$$
Y_{t}=\left(\alpha_{1}+\beta_{1} Y_{t-1}\right)\left(1-G\left(s_{t} ; \gamma, c\right)\right)+\left(\alpha_{2}+\beta_{2} Y_{t-1}\right) G\left(s_{t} ; \gamma, c\right)+\varepsilon_{t},
$$

where popular choice for $G$ is the logistic function given by

$$
G\left(s_{t} ; \gamma, c\right)=\left(1+\exp \left\{-\gamma\left(s_{t}-c\right)\right\}\right)^{-1}
$$

with $s_{t}$ being a state variable, $c$ the threshold value and $\gamma$ the smoothness parameter. When $s_{t}=Y_{t-d}$ (3.29) is called self-exiting STAR or SETAR and this is the model we consider here with $d=1$.

An advantage of using the copula based tests for the null of symmetric time dependence is that although the corresponding Copula Markov models are in general non-linear and asymmetric they nest symmetry as a special case. Besides we do not have to deal with problems of unidentified 
parameters in the logistic function under the null hypothesis like the classical tests for STAR models relying on Taylor expansions.

It is rather obvious that the simple first order STAR generates asymmetric dependence between $Y_{t}$ and $Y_{t-1}$ as long as $\beta_{1} \neq \beta_{2}$. In Figure 3.5 we illustrate the degree of asymmetry generated by this DGP in terms of the difference between the $\tau^{+}$and $\tau^{-}$. The parameter choices are $\alpha_{1}=\alpha_{2}=0$, $\beta_{1}=0.4, c=1$ and $\gamma=2.5$. $\beta_{2}$ ranges from 0.4 to 0.9 and $\varepsilon_{t} \sim N(0,1)$. One can see that a significant degree of asymmetry is present even for small differences between $\beta_{1}$ and $\beta_{2}$.

\subsection{Monte Carlo studies}

In this section we provide an extensive Monte Carlo study to assess the quality of the copula based tests for asymmetric dependence. In the first subsection we study the size and power of the tests and compare it to the test of Hong et al. (2007). In the other three subsections we study the behavior of the copula based tests for the three DGPs studied in Section 3.3. Note that for the simulations in section 3.4.1 we use the critical values based on the asymptotic distribution in Vuong (1989), whereas in sections 3.4.2 to 3.4.4 we used the ones proposed by Chen and Fan (2006a) accounting for the use of the empirical CDF.

\subsubsection{The general case}

In order to study the properties of the Gumbel mixture (GM) and Clayton mixture (CM) tests in comparison to the $J$-test for asymmetric correlation proposed by Hong et al. (2007) under misspecification of the model and fixed alternatives we chose a data generating process that is quite similar to the one used by these authors in their simulation. ${ }^{6}$ We perform the tests for the null hypothesis of symmetric dependence on i.i.d. $U(0,1)$ data generated by the following copula model.

$$
C(u, v)=\alpha \cdot C_{\theta_{1}}^{\text {Clayton }}(u, v)+(1-\alpha) \cdot C_{\theta_{2}}^{\text {Normal }}(u, v)
$$

$\theta_{1}$ and $\theta_{2}$ are chosen such that the overall Kendall's tau of the mixture is equal to 0.5. This DGP is characterized by symmetric dependence when $\alpha=0$, but asymmetric dependence increases as $\alpha$ get larger. We let the mixing parameter $\alpha$ take vary for 0 to 1 over a grid of 0.05 and we consider 4 different sample sizes, namely $(100,250,500,1000)$. The power curves based on 1000 Monte Carlo

\footnotetext{
${ }^{6}$ Hong et al. (2007) demonstrated in their paper that the $J$-test is superior to the $H^{2}-$ test proposed by Ang and Chen (2002), so we do not consider it here.
} 
Figure 3.6: Power functions of the CM, CM and $J$-test as a function of $\alpha$ in (3.30)
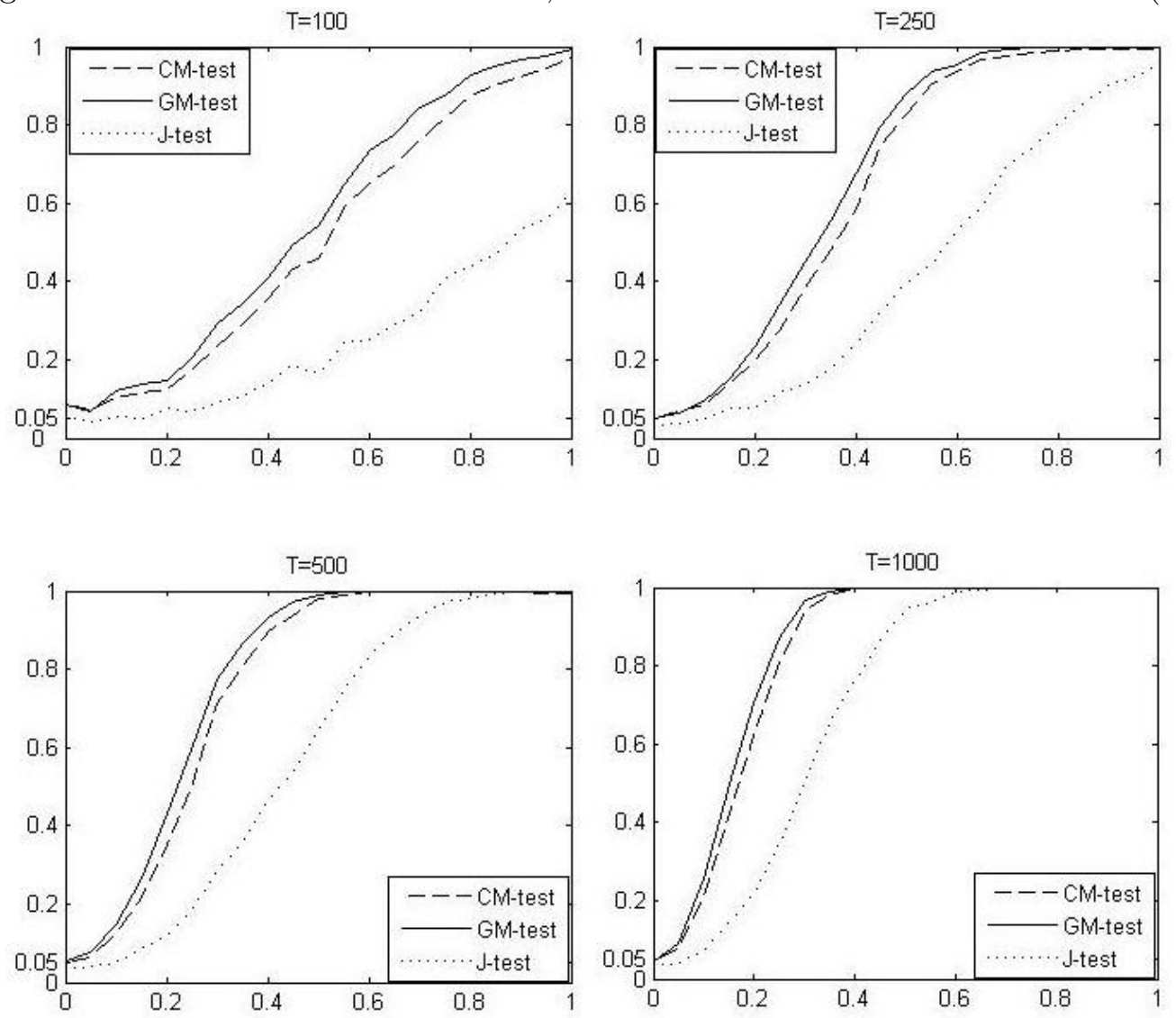

simulations are reported in Figure 3.6. Note that except for the case of 100 observations the CM and GM tests have size that is extremely close to the nominal level of $5 \%$, whereas the $J$-test is slightly undersized. The power of the copula based tests is always significantly higher than that of the $J$-test, the GM-test performing slightly better. As mentioned above, the reason for the copula based tests to outperform the $J$-test is most likely that they rely on a more robust measure of dependence and that these tests use the full information of the sample, whereas the $J$-test only uses those observations that are jointly above and below the exceedance levels. Note that the $J$-test has been performed on the $U(0,1)$ variables, so it actually tests the symmetry of the rank correlation coefficient also known as Spearman's $\rho$. When we transformed the data into $N(0,1)$ variables the $J-t e s t$ is severely undersized (below 1\%) and has lower power. The copula based tests circumvent this problem by automatically standardizing the data with the empirical probability integral transform.

When using the critical values from a $\chi_{2}^{2}$ distribution instead of the correct ones from a mixture of $\chi_{1}^{2}$ distributions the copula based tests turn out to be slightly undersized and have less power, but 
Table 3.1: Rejection frequency of the null of symmetric dependence for data generated by (3.23)

\begin{tabular}{lrrrr}
\hline \hline$\sigma_{h}=0.01$ & $\sigma_{l}=0.01$ & & & \\
GM-test & $\rho_{l}=0.4$ & $\rho_{l}=0.5$ & $\rho_{l}=0.6$ & $\rho_{l}=0.7$ \\
$\mathrm{~T}=100$ & 0.081 & 0.133 & 0.241 & 0.465 \\
$\mathrm{~T}=250$ & 0.082 & 0.252 & 0.500 & 0.804 \\
$\mathrm{~T}=500$ & 0.114 & 0.453 & 0.853 & 0.989 \\
$\mathrm{~T}=1000$ & 0.227 & 0.773 & 0.987 & 1.000 \\
\hline $\mathrm{CM}$-test & & & & \\
$\mathrm{T}=100$ & 0.033 & 0.054 & 0.119 & 0.266 \\
$\mathrm{~T}=250$ & 0.057 & 0.189 & 0.400 & 0.729 \\
$\mathrm{~T}=500$ & 0.104 & 0.395 & 0.809 & 0.982 \\
$\mathrm{~T}=1000$ & 0.185 & 0.735 & 0.980 & 1.000 \\
\hline$\sigma_{h}=0.01$ & $\sigma_{l}=0.02$ & & & \\
$\mathrm{GM}-\mathrm{test}$ & $\rho_{l}=0.4$ & $\rho_{l}=0.5$ & $\rho_{l}=0.6$ & $\rho_{l}=0.7$ \\
$\mathrm{~T}=100$ & 0.322 & 0.401 & 0.530 & 0.608 \\
$\mathrm{~T}=250$ & 0.509 & 0.628 & 0.802 & 0.929 \\
$\mathrm{~T}=500$ & 0.816 & 0.928 & 0.986 & 0.997 \\
$\mathrm{~T}=1000$ & 0.985 & 1.000 & 1.000 & 0.998 \\
\hline $\mathrm{CM}$-test & & & & \\
$\mathrm{T}=100$ & 0.224 & 0.276 & 0.374 & 0.464 \\
$\mathrm{~T}=250$ & 0.490 & 0.584 & 0.755 & 0.872 \\
$\mathrm{~T}=500$ & 0.780 & 0.905 & 0.982 & 0.993 \\
$\mathrm{~T}=1000$ & 0.987 & 1.000 & 1.000 & 1.000 \\
\hline \hline
\end{tabular}

Note: Table 3.1 reports the rejection frequency of the null hypothesis symmetric dependence using the two copula based tests. The data has been generated by (3.23) with $\mu_{h}=0.01$ and $\mu_{l}=-0.01$. The change in regime always occurs at $T / 2$. The number of Monte Carlo replications is fixed to 1000 .

the tests still perform better than the $J-$ test. $^{7}$

\subsubsection{Bull and bear markets}

Data is generated from the bull and bear markets model (3.23). We consider two different cases, one where volatility remains constant across states and one where it increases during the bear state. The mean is chosen to be the same for both markets, namely $\mu_{h}=0.01$ and $\mu_{l}=-0.01$. The standard deviation $\sigma_{s}$ is equal to 0.01 . In the case that it increases during the bear state it is equal to 0.02 . The change in regime always occurs at $\mathrm{T} / 2$, where $\mathrm{T}$ is the sample size, which takes on the values $(100,250,500,1000)$. Finally, $\rho_{h}$ is chosen to be 0.3 , whereas $\rho_{l}$ varies across the values $(0.4,0.5$, $0.6,0.7)$. Before applying our tests we transform the two artificially generated return series into $U(0,1)$ variables by the empirical probability integral transform. Table 3.1 shows that the two tests are able to identify the asymmetric dependence reasonably well, as long as the change in correlation

\footnotetext{
${ }^{7}$ Results are available upon request.
} 
is large enough. The GM-test has higher power than the CM-test in all situations. It is notable that a change in variance additionally to a change in correlation strongly increases the asymmetry. This can be explained by the reasoning of Forbes and Rigobon (2002) that changes in volatility lead to spurious increases in observed correlation combined with our calculations in Section 3.3.

\subsubsection{Nonlinear VAR}

We extend the DGP (3.24) by introducing an intercept and some dynamics, so that it becomes the following simple nonlinear vector autoregression $(\mathrm{VAR})^{8}$

$$
\begin{aligned}
& X_{t}=\alpha_{1}+\beta_{1} X_{t-1}+\beta_{2} Y_{t-1}+\delta I\left(\epsilon_{2 t}>c\right)+\epsilon_{1 t} \\
& Y_{t}=\alpha_{2}+\gamma_{1} X_{t-1}+\gamma_{2} Y_{t-1}+\delta I\left(\epsilon_{1 t}>c\right)+\epsilon_{2 t} .
\end{aligned}
$$

The parameter choices are $\alpha_{1}=0.2, \alpha_{2}=0.1, \beta_{1}=0.3, \beta_{2}=0.1, \gamma_{1}=0.1$ and $\gamma_{2}=0.3$. The innovations are drawn from a standard multivariate normal distribution with correlation coefficient equal to 0.5 . The threshold value $c$ takes on the values $(0,0.5,1,1.5)$, the effect of the threshold $\delta$ varies over $(0.3,0.5,0.7,0.9)$ and the sample sizes we consider are $(100,250,500,1000)$. A linear $\operatorname{VAR}(1)$ is fit to the data and the residuals are collected. After transforming them into $U(0,1)$ variables by the empirical probability integral transform the two copula based tests are performed. As before, the number of Monte Carlo replications is equal to 1000. The results can be found in Table 3.2. The rejection frequency of the null of symmetric dependence increases with $\delta$ and with the sample size. It also depends on $c$ and is highest for $c=1$. That makes intuitively sense, as a larger threshold means that less observations exceed it and thus the effect of the indicator function occurs less often. Even after the introduction of intercepts and dynamics it still holds that when $c=0$, the DGP does not cause any asymmetric dependence, confirming that the findings in example 2 still hold after the DGP is generalized. Finally, the GM-test again outperforms the CM-test in terms of power.

\subsubsection{SETAR models}

In this final Monte Carlo simulation we illustrate how the tests for asymmetric dependence can be used to distinguish nonlinear autoregressive processes from linear ones. We generate a time series from DGP (3.29). The intercept $\alpha$ is chosen equal to $0.2, \beta_{1}=0.4$ and the innovations come from a

\footnotetext{
${ }^{8}$ Strictly speaking this is a linear VAR, as the nonlinearity only enters through the innovations, which are martingale differences. As mentioned before, the DGP is comparable to the model suggested by Pesaran and Pick (2007), but it is easier to analyze.
} 
Table 3.2: Rejection frequency of the null of symmetric dependence for data generated by (3.31)

\begin{tabular}{lrrrrrrrr}
\hline \hline CM-test & & & \multicolumn{5}{c}{ GM-test } \\
$\mathrm{T}=100$ & $\mathrm{c}=0$ & $\mathrm{c}=0.5$ & $\mathrm{c}=1$ & $\mathrm{c}=1.5$ & $\mathrm{c}=0$ & $\mathrm{c}=0.5$ & $\mathrm{c}=1$ & $\mathrm{c}=1.5$ \\
$\delta=0.3$ & 0.045 & 0.097 & 0.156 & 0.122 & 0.102 & 0.220 & 0.297 & 0.292 \\
$\delta=0.5$ & 0.032 & 0.177 & 0.366 & 0.233 & 0.064 & 0.303 & 0.577 & 0.451 \\
$\delta=0.7$ & 0.027 & 0.269 & 0.582 & 0.362 & 0.059 & 0.429 & 0.767 & 0.609 \\
$\delta=0.9$ & 0.026 & 0.396 & 0.773 & 0.496 & 0.063 & 0.525 & 0.893 & 0.732 \\
\hline $\mathrm{T}=250$ & & & & & & & & \\
$\delta=0.3$ & 0.033 & 0.223 & 0.379 & 0.208 & 0.047 & 0.251 & 0.417 & 0.259 \\
$\delta=0.5$ & 0.043 & 0.455 & 0.781 & 0.478 & 0.052 & 0.546 & 0.833 & 0.570 \\
$\delta=0.7$ & 0.037 & 0.705 & 0.900 & 0.733 & 0.043 & 0.783 & 0.970 & 0.810 \\
$\delta=0.9$ & 0.030 & 0.865 & 0.953 & 0.869 & 0.04 & 0.908 & 0.992 & 0.931 \\
\hline $\mathrm{T}=500$ & & & & & & & & \\
$\delta=0.3$ & 0.032 & 0.435 & 0.686 & 0.412 & 0.050 & 0.484 & 0.715 & 0.461 \\
$\delta=0.5$ & 0.046 & 0.812 & 0.968 & 0.852 & 0.054 & 0.870 & 0.983 & 0.892 \\
$\delta=0.7$ & 0.047 & 0.961 & 0.985 & 0.969 & 0.059 & 0.979 & 0.996 & 0.985 \\
$\delta=0.9$ & 0.054 & 0.997 & 0.983 & 0.987 & 0.068 & 0.997 & 0.998 & 0.996 \\
\hline $\mathrm{T}=1000$ & & & & & & & & \\
$\delta=0.3$ & 0.038 & 0.732 & 0.942 & 0.727 & 0.050 & 0.750 & 0.944 & 0.783 \\
$\delta=0.5$ & 0.043 & 0.995 & 1.000 & 0.989 & 0.050 & 0.994 & 1.000 & 0.994 \\
$\delta=0.7$ & 0.052 & 1.000 & 0.997 & 0.998 & 0.048 & 1.000 & 0.999 & 0.998 \\
$\delta=0.9$ & 0.034 & 1.000 & 1.000 & 0.997 & 0.044 & 0.999 & 0.999 & 1.000 \\
\hline \hline
\end{tabular}

Note: Table 3.2 reports the rejection frequency of the null hypothesis symmetric dependence using the two copula based tests. The data has been generated by (3.31) with $\alpha_{1}=0.2, \alpha_{2}=0.1, \beta_{1}=0.3, \beta_{2}=0.1, \gamma_{1}=0.1$ and $\gamma_{2}=0.3$. The number of Monte Carlo replications is fixed to 1000.

standard normal distribution. We vary both the parameter $\beta_{2}$, which can take on the values $(0.5,0.6$, 0.7 ), the threshold $c$ (values are 0,1 and 2) and the smoothness parameter $\gamma$ of the logistic function, which is varies over $(1,2.5,3.5,10)$. The series $Y$ is transformed into a standard uniform variable and the asymmetry tests are applied to $Y_{t}$ and $Y_{t-1}$. For spatial reasons we report the outcomes, which can be found in Table 3.3, only for the case of 1000 observations. The tests are able to capture the nonlinearities reasonably well. The size of the tests (not reported) is very close to the nominal level of $5 \%$. As a comparison we also performed a test based on a third order Taylor expansions that was designed for logistic STAR models in the same simulation setup (van Dijk et al. 2002). The results, which are available upon request, showed that the latter test in fact has slightly less power than the GM-test and the CM-test, although one has to keep in mind that the copula based test only can be used for SETAR models of order one, whereas the test designed for STAR models is applicable in more general situations. 
Table 3.3: Rejection frequency of the null of symmetric dependence for data generated by (3.29)

\begin{tabular}{rrrrrrrrr}
\hline \hline CM-test & & & \multicolumn{7}{c}{ GM-test } \\
$\beta_{2}=0.5$ & $\gamma=1$ & $\gamma=2.5$ & $\gamma=3.5$ & $\gamma=10$ & $\gamma=1$ & $\gamma=2.5$ & $\gamma=3.5$ & $\gamma=10$ \\
$c=0$ & 0.136 & 0.176 & 0.155 & 0.147 & 0.158 & 0.183 & 0.181 & 0.161 \\
$c=1$ & 0.143 & 0.215 & 0.226 & 0.234 & 0.174 & 0.229 & 0.249 & 0.257 \\
$c=2$ & 0.112 & 0.167 & 0.178 & 0.208 & 0.124 & 0.199 & 0.200 & 0.232 \\
\hline$\beta_{2}=0.6$ & & & & & & & & \\
$c=0$ & 0.460 & 0.465 & 0.489 & 0.441 & 0.492 & 0.499 & 0.528 & 0.475 \\
$c=1$ & 0.467 & 0.681 & 0.694 & 0.708 & 0.515 & 0.702 & 0.712 & 0.721 \\
$c=2$ & 0.376 & 0.565 & 0.618 & 0.693 & 0.426 & 0.604 & 0.651 & 0.738 \\
\hline$\beta_{2}=0.7$ & & & & & & & & \\
$c=0$ & 0.812 & 0.810 & 0.789 & 0.775 & 0.853 & 0.855 & 0.845 & 0.817 \\
$c=1$ & 0.880 & 0.976 & 0.982 & 0.964 & 0.900 & 0.979 & 0.984 & 0.970 \\
$c=2$ & 0.736 & 0.937 & 0.968 & 0.979 & 0.772 & 0.947 & 0.971 & 0.982 \\
\hline \hline
\end{tabular}

Note: Table 3.3 reports the rejection frequency of the null hypothesis symmetric dependence between $Y_{t}$ and $Y_{t-1}$ using the two copula based tests. The data has been generated by (3.29) with $\alpha=0.2$ and $\beta_{1}=0.4$. The number of observations is equal 1000 to and the number of Monte Carlo replications is fixed to 1000 .

\subsection{Empirical applications}

In the section we apply the tests for asymmetric dependence two different data set. First we test for asymmetric dependence between monthly returns of 17 international stock markets, which relates to example 1 in Section 3.3. Second the tests are applied on macroeconomic data. The tests are used to test both for asymmetric dependence over time of the individual series as suggested in example 3 and for asymmetric dependence of the residuals of a linear and nonlinear VAR motivated by example 2 .

Critical values used in this section are computed using the asymptotic distribution derived in Chen and Fan (2006a) in order to account for estimating the marginal distributions with the empirical CDF.

\subsubsection{International stock market returns}

The first data set we consider are monthly stock market returns for the most important stock markets in the world over a period of over 30 years spanning the period from January 1974 to December 2006. This amount to a total of 396 observations per series and a total of 136 pairs of countries. ${ }^{9}$ The data has been obtained from Datastream. The two tests we have introduced are applied to each of the 136 pairs of de-meaned stock market returns, which have been filtered using a GARCH $(1,1)$ model to account for conditional heteroscedasticity. The fraction of rejections of the null hypothesis

\footnotetext{
${ }^{9}$ The countries we consider are Australia, Austria, Belgium, Canada, Denmark, France, Germany, Hong Kong, Ireland, Italy, Japan, Netherlands, South Africa, Singapore, Switzerland, UK and USA.
} 
Table 3.4: Application on international stock market returns

\begin{tabular}{lrrrr}
\hline \hline & GM-test & CM-test & avg. $\hat{\tau}^{+}$ & avg. $\hat{\tau}^{-}$ \\
Mean rejection and cond. $\tau$ & 0.647 & 0.552 & 0.119 & 0.250 \\
\hline \hline
\end{tabular}

Note: Columns 2 and 3 report the fraction of pairs of stock market returns for which the null of symmetric dependence is rejected at a $5 \%$ confidence level using the GM- and CM-test. Columns 4 and 5 report the average measure of $\tau^{+}$ and $\tau^{-}$.

of symmetric dependence at a 5\% confidence level is reported in Table 3.4 together with average estimates of $\tau^{+}$and $\tau^{-}$. The outcomes do not only suggest that the majority of pairs is characterized by asymmetric dependence, but also that the dependence is higher for negative returns than it is for positive returns. These findings do not come as a surprise and similar ones have been documented in the literature mentioned in the introduction, as well as in many other studies using copulas or conditional correlation measures. Thus we consider a further field of application of our tests.

\subsubsection{Application on US output and unemployment data}

The second data set we consider to illustrate our testing procedure are quarterly observations of US GNP at 1982 prices and total unemployment ranging from 1951 until 1990. In order to have stationary data the log difference has been taken of the output series and the first difference of the unemployment series, leading to a total of 156 observations. The data have been used by Altissimo and Violante (2001), who modeled them with a threshold VAR. The first step of our analysis is testing for asymmetries in the autoregressive dynamics of the two individual series. In a preliminary analysis AR models have been fit to the data to check the order of the dynamics present. This is an important step, as our approach only is valid if $Y_{t}$ can be treated as a first order Markov process. Ignoring dependencies with higher lags could severely influence the results. For both series only the first order autoregressive term was significant, which means we do not have to worry about omitted higher order dynamics when using copulas to mimic the AR(1) process. The data have been transformed into $U(0,1)$ variables using the empirical probability integral transform and the tests for symmetry are applied on the transformed variables $\hat{u}_{t}$ and $\hat{u}_{t-1}$. Table 3.5 presents the p-values of the tests along with the sample estimates of $\tau^{+}$and $\tau^{-}$. Symmetric dependence is rejected for the unemployment series at a 5\% confident level. For the change in output we cannot reject the null of symmetric dependence. This means that when modeling the two series by AR(1) models the change in output can be well described by a linear model, whereas the change in unemployment series should be modeled by a non-linear specification such as the STAR model. The findings for the GNP are in contrast to Potter (1995) who, using a slightly different sample period, included more lags in 
Table 3.5: Tests for asymmetric dependence on the autoregressive dynamics of Unemployment and GNP

\begin{tabular}{lrrrr}
\hline \hline & GM-test & CM-test & $\hat{\tau}^{+}$ & $\hat{\tau}^{-}$ \\
Change in unemployment & 0.007 & 0.010 & 0.363 & 0.255 \\
Change in log GNP & 0.579 & 0.503 & 0.073 & 0.113 \\
\hline \hline
\end{tabular}

Note: Table 3.5 reports the p-values of the null hypothesis symmetric dependence and the sample estimates of $\tau^{+}$and $\tau^{-}$between the observations at time $t$ and time $t-1$ for the change in unemployment and change in log GNP using data from the US ranging from 1951:Q1 to 1990:Q4.

Table 3.6: Tests for asymmetric dependence on the residuals of a linear and nonlinear VAR

\begin{tabular}{lrrrr}
\hline \hline & GM-test & CM-test & $\hat{\tau}^{+}$ & $\hat{\tau}^{-}$ \\
linear VAR & 0.001 & 0.000 & 0.032 & 0.364 \\
nonlinear VAR & 0.425 & 0.052 & -0.017 & 0.012 \\
\hline \hline
\end{tabular}

Note: Table 3.6 reports the p-values of the null hypothesis symmetric dependence and the sample estimates of $\tau^{+}$ and $\tau^{-}$between the residuals of a linear $\operatorname{VAR}(2)$ and a $\operatorname{VAR}(2)$ augmented with two lags of the variable CDR defined in (3.32) for the change in log GNP and change in unemployment using data from the US ranging from 1951:Q1 to 1990:Q4

the autoregressive specification (most of which were not significant), and who found evidence for a Self-Exciting Threshold Autoregression. Next, we test for asymmetric dependence of the residuals of a VAR model for the two series. Following Altissimo and Violante (2001) we chose a lag length of 2 to capture the dynamics. We consider two specifications of the VAR. The first one is the standard linear one, whereas the second one is the threshold VAR specification that was used by Altissimo and Violante (2001). For the nonlinear specification the VAR is augmented with 2 lags of the following feedback variable

$$
C D R_{t}(r, k)=y_{t}-\max \left(y_{t}, y_{t-1}+r, \ldots, y_{t-k}+r\right),
$$

where $y$ is the real GNP. The variable captures periods where output is lower than the maximum in past periods increased by a threshold $r$, so it identifies recession periods. The threshold was determined endogenously by Altissimo and Violante (2001) and turned out to be -0.138 and we set $k=10$. Table 3.6 reports the outcomes of the tests and the sample estimates of $\tau^{+}$and $\tau^{-}$. Since the residuals are negatively correlated we actually perform the analysis using minus the second residual to ensure the tests can still be applied. For the residuals of the linear VAR we clearly reject the null of symmetric dependence. When the two lags threshold variable are included this is not the case anymore.

Overall these results are in line with Altissimo and Violante (2001). They found that nonlinear 
dynamics are only present in the output equation and that the nonlinearity of the unemployment series "transmits to output purely through its cross correlation". This finding can also explain the difference of our specification to the one by Potter (1995).

\subsection{Conclusion}

Various kinds of asymmetries and nonlinearities are frequently observed in economic and financial data. These may be hard to distinguish from one another in a given data set. In this chapter we have proposed a way to test for a specific type of nonlinearity, namely asymmetric dependence structures. We defined a conditional version of Kendall's tau as the probability of concordance minus the probability of discordance of a pair of random variables conditional on both variables being above or below their respective medians. This adjusted dependence measure serves as a basis for our definition and tests of asymmetric dependence based either on the Gumbel or the Clayton copula. The conditional dependence structures of these two copulas are analyzed more carefully than has been done in previous studies. The tests we propose have good size properties and clearly outperform existing approaches for the same problem in terms of power.

Different data generating processes may lead to the observation of asymmetric dependencies. This highlights an important issue: What do I know if I reject symmetric dependence? The answer depends on the situation at hand and can not be generally answered. Often it can be a sign of misspecification of an underlying model such as ignoring different regimes, structural breaks, time varying parameters, changing volatilities and leverage effects. In other cases it may point to a feature in the data that cannot be easily explained, but that should nevertheless be regarded when e.g. making investment decisions. In any case it tells us that a linear approximation may not capture the important aspects of the data in a satisfactory way. This may mean that linear correlation is not the appropriate measure of (unconditional) stock market dependence, that the autoregressive dynamics in the change in unemployment are not sufficiently captured by a linear AR model or that a linear VAR is not enough to capture the joint dynamics of the changes in output and unemployment.

\section{A Proof of Proposition 3.1}

We start by proving the general formula

$$
\tau_{C}=4 E(C(U, V))-1
$$


Let $(U, V) \sim C$, where $C$ is a Copula. Take an arbitrary pair $(u, v)$ drawn from Copula $C$. We are interested in the probability that a second pair $\left(u^{\prime}, v^{\prime}\right)$ drawn from $C$ is concordant with the first pair minus the probability that the two pairs are discordant, i.e. in the quantity $P[(u-U)(v-V)>$ $0]-P[(u-U)(v-V)<0]$. Dividing the unit square into four regions we have concordance for points in the rectangles southwest (which we call $\mathrm{A}$ ) and northeast $(\mathrm{C})$ of $(u, v)$ and discordance in the rectangles southeast (B) and northwest (D) of $(u, v)$. The probability of an observation in each of the rectangles is given by its H-Volume (see Nelsen, 2006 for a definition):

$$
\begin{aligned}
& P(A)=C(u, v)+C(0,0)-C(u, 0)-C(0, v) \\
& P(C)=C(1,1)+C(u, v)-C(u, 1)-C(1, v) \\
& P(B)=C(1, v)+C(u, 0)-C(1,0)-C(u, v) \\
& P(D)=C(u, 1)+C(0, v)-C(0,1)-C(u, v)
\end{aligned}
$$

The probability of concordance minus the probability of discordance is equal to $P(A)+P(C)-$ $P(B)-P(D)$. Noting the basic properties that a copula is grounded $(C(a, 0)=C(0, a)=0$ for any $a)$ and that it has margins $(C(b, 1)=C(1, b)=b$ for any $b)$ we find that

$$
P(A)+P(C)-P(B)-P(D)=4 C(u, v)-2 v-2 u+1 .
$$

Integrating this expression over all possible points $(u, v)$ over the unit square with respect to the underlying copula and noting that the mean of a standard uniform random variable is 0.5 the formula in (3.33) follows.

Now take an arbitrary pair $(u, v)$ such that $u>0.5$ and $v>0.5$. The joint probability that a second pair $\left(u^{\prime}, v^{\prime}\right)$ from $C$ is concordant with $(u, v)$ and that both coordinates are greater than 0.5 minus the joint probability that the second point is discordant with $(u, v)$ and both coordinates are greater than 0.5, i.e. $P[(u-U)(v-V)>0, U>0.5, V>0.5]-P[(u-U)(v-V)<0, U>0.5, V>0.5]$, is given by

$$
4 C(u, v)-2 C(u, 0.5)-2 C(0.5, v)-2 u-2 v+2+C(0.5,0.5)
$$

Note that the probability that both coordinates of a pair from $C$ are larger than $0.5, P[u>0.5, v>$ $0.5]$, is equal to $C(0.5,0.5)$ and the conditional probability is the joint probability divided by the 
marginal probability. Then the conditional probability of concordance minus the conditional probability discordance is equation $(3.34)$ divided by $C(0.5,0.5)$. Integrating this over all points $(u, v)$ such that $u>0.5$ and $v>0.5$ and noting that for a $U(0,1)$ variable $E(U \mid U>0.5)=0.75$, the expression for $\tau^{+}$follows.

The formula for $\tau^{-}$is derived in a similar way and its derivation is omitted. 


\section{Time-varying copulas: a survey ${ }^{1}$}

\subsection{Introduction}

It is well accepted that the hypothesis of (multivariate) normality is one that is usually not supported by the data for many types of variables. This has created the need to construct flexible, non-standard multivariate distributions and this task can easily be solved using a class of functions known as copulas (Sklar 1959). Any multivariate distribution function can be decomposed into the marginal distributions that describe the individual behavior of each series and the copula that fully captures the dependence between the variables. Furthermore, given a set of marginal distributions and a copula a multivariate distribution can be constructed by coupling the marginals with the copula. The flexibility of the way dependencies can be modeled independently of the marginal distributions has made copulas particularly popular for financial applications. The most important fields of applications are pricing CDO's (Li 2000), calculating the Value-at-Risk of a portfolio (Embrechts, Höing, and Juri 2003, Giacomini, Härdle, and Spokoiny 2009), the pricing of options with multiple underlying assets (van den Goorbergh et al. 2005) or portfolio construction (Patton 2004). Textbook treatments of the theory of copulas are given in Joe (1997) and Nelsen (2006). The book Cherubini et al. (2004) deals entirely with various applications of copulas in finance.

Most of the time copulas are applied to financial time series data, but often they are treated to be constant over time. However, it has become a stylized fact that correlations between asset returns are not constant through time, a finding that has been documented by, among many others, Erb, Harvey, and Viskante (1994), Longin and Solnik (1995) or Engle (2002). Some notable parametric models to model these time-varying correlations in multivariate volatility models are the DCC GARCH model, simultaneously proposed by Engle (2002) and Tse and Tsui (2002), a stochastic volatility model with stochastic correlations by $\mathrm{Yu}$ and Meyer (2006) and the regime switching model for dynamic correlations by Pelletier (2006). Hafner, van Dijk, and Franses (2006) propose a semiparametric model for correlation dynamics.

\footnotetext{
${ }^{1}$ This chapter is based on Manner and Reznikova (2009).
} 
Even though copulas allow for more general dependence structures than simple linear correlation it seems unrealistic to treat dependence as constant, given that correlations have been found to be time-varying. To our knowledge the first papers allowing copulas to be time-varying were Patton (2006b), who extended Sklar's theorem for conditional distributions and proposed a parametric model to describe the evolution of the copula parameter, and Dias and Embrechts (2004) who proposed a test for structural breaks in the copula parameter. Subsequently, a large number of studies has dealt with the application of time-varying copulas and the development of new models and tests to appropriately model time-varying dependencies. Some contributions to this fast growing field of research are van den Goorbergh et al. (2005), Jondeau and Rockinger (2006), Giacomini et al. (2009), Guégan and Zhang (2009), Chollete, Heinen, and Valdesogo (2009), Creal, Koopman, and Lucas (2008), Hafner and Manner (2008) and Hafner and Reznikova (2008).

In this chapter we want to offer a survey over the existing models for time-varying copulas by focusing on the specification, estimation and properties of a number of models. Furthermore, we discuss how the best fitting time-varying copula can be chosen among a number of competing ones and how the goodness-of-fit of a candidate model can be tested. A Monte Carlo study compares the performance of the model selection and goodness-of-fit criteria for competing specifications of dynamics of the copula parameter, and shows how well the competing time-varying copula models are able to estimate the (latent) dependence process. In an empirical application alternative models are estimated for two financial data sets and in addition to statistical model selection the ability of the models to correctly estimate the Value-at-Risk is tested.

The rest of the chapter is organized as follows. In Section 4.2 copulas and their estimation are reviewed. Section 4.3 provides a survey over existing time-varying copula models followed by a simulation study in Section 4.4. An empirical application is provided in Section 4.5 and, finally, Section 4.6 provides conclusions and an outlook to future developments.

\subsection{Copulas}

In this section we shortly discuss the basic theory of copulas and some ways to estimate their parameters. For a complete introduction to copulas see Joe (1997).

Lets consider the bivariate stochastic process $\left\{X_{t}\right\}_{t=1}^{T}$ with $X_{t}=\left(X_{1 t}, X_{2 t}\right)^{\prime}$. Let $F\left(X_{1 t}, X_{2 t}\right)$ be the joint distribution, whereas $F_{i}$ and $f_{i}$ will denote the marginal $c d f$ and $p d f$ respectively for $i=1,2$. Then by Sklar's theorem there exists a copula function $C(\cdot, \cdot):[0,1]^{2} \rightarrow[0,1]$ mapping the 
marginal distributions of $X_{1 t}$ and $X_{2 t}$ to their joint distribution through

$$
F\left(X_{1 t}, X_{2 t}\right)=C\left(F_{1}\left(X_{1 t}\right), F_{2}\left(X_{2 t}\right)\right) .
$$

We assume that the marginals can be modeled parametrically, thus the probability transform is given by $U_{i t}=F_{i}\left(X_{i t} ; \phi_{i}\right)$, where $\phi_{i}$ is the vector of parameters completely describing the individual behavior of the series. $F_{i}\left(X_{i t} ; \phi_{i}\right)$ can be a conditional distribution and in financial econometrics $X_{i t}$ is usually modeled by an ARMA-GARCH type model, whose residuals are treated as iid random variables. We also assume that the copula belongs to a parametric family $C_{\theta}, \theta \in \Theta \subset \mathbb{R}^{K}$. Some examples of parametric copulas are given in the appendix.

Given that the copula function and the marginals are absolutely continuous, the following expression for the joint $p d f$ holds

$$
f\left(X_{1 t}, X_{2 t}\right)=c\left(U_{1 t}, U_{2 t} ; \theta\right) \prod_{i=1}^{2} f_{i}\left(X_{i t} ; \phi_{i}\right),
$$

where $c(\cdot, \cdot)$ denotes the copula density. Assume a sample $X_{t}, t=1, \ldots, T$. The log-likelihood function is given by

$$
\begin{aligned}
L(\theta, \phi) & =\sum_{t=1}^{T}\left\{\log c\left(U_{1 t}, U_{2 t} ; \theta\right)+\log f_{1}\left(X_{1 t} ; \phi_{1}\right)+\log f_{2}\left(X_{2 t} ; \phi_{2}\right)\right\} \\
& =L_{C}(\theta, \phi)+L_{X_{1}}\left(\phi_{1}\right)+L_{X_{2}}\left(\phi_{2}\right),
\end{aligned}
$$

where $\phi=\left(\phi_{1}^{\prime}, \phi_{2}^{\prime}\right)^{\prime}$. Thus, the full log-likelihood function $L(\theta, \phi)$ can be split into two parts, copula likelihood $L_{C}(\theta, \phi)$ and likelihood of the marginals $L_{X_{1}}\left(\phi_{1}\right)$ and $L_{X_{2}}\left(\phi_{2}\right)$.

There are several ways to estimate $\theta$ and $\phi$. One possible method is to estimate the parameters simultaneously by full maximum likelihood

$$
(\widehat{\theta}, \widehat{\phi})=\underset{\theta, \phi}{\arg \max } L(\theta, \phi) .
$$

This estimation method is conceptually straightforward. However, in some situations it may be computationally rather burdensome.

Another approach is to use a two stage estimator. At the first stage only the parameters from the marginal distributions are estimated

$$
\widehat{\phi}_{i}=\underset{\phi}{\arg \max } L_{X_{i}}\left(\phi_{i}\right), i=1,2 .
$$


At the second stage the dependence parameter is estimated from the copula likelihood

$$
\widehat{\theta}=\underset{\theta}{\arg \max } L_{C}(\theta, \widehat{\phi})
$$

However, the estimation of the parameters in two steps leads to a loss in efficiency and standard errors cannot be obtained as the inverse of the Fisher Information Matrix anymore. By applying one step of the Newton-Rhapson algorithm to the full likelihood function using the two step estimators, statistical efficiency can be achieved (see van der Vaart (1998), Ch.5).

Alternatively when the marginal model is unknown Genest et al. (1995) suggest modeling the marginal distribution with the empirical $c d f$ and estimating the copula on the ranks of the data. Again, the problem of loss of efficiency occurs and calculation of the standard errors of the estimated copula parameter is quite tedious. On the other hand, this method is robust to the misspecification of the marginals, which can cause biased estimates of the copula parameter.

\subsection{Survey}

In this section we will give an overview of the time-varying copula models that have been proposed in the literature. We focus our attention on the specification of the dynamics of the copula parameter and the estimation of the models. For the sake of brevity a complete description of the properties and many details of the procedures involved must be omitted. The interested reader is referred to the original papers.

Note that the following paragraphs describe only the specification and estimation of the copula, whereas the marginals are assumed to be appropriately modeled and the data is assumed to be transformed into the $U(0,1)$ variables $U_{1 t}$ and $U_{2 t}$. In general the time-varying dependence parameter of the copula will be called $\theta_{t}$, and for the correlation coefficient of the Gaussian copula $\rho_{t}$ is reserved.

\subsubsection{Observation driven models}

Patton (2006b) and Creal et al. (2008) propose similar observation driven copula models for which the time-varying dependence parameter of a copula is a parametric function of transformations of the lagged data and an autoregressive term.

The model of Patton for the dynamics of the correlation for Gaussian or Student copula has the 
following form,

$$
\begin{aligned}
\rho_{t} & =\Lambda_{1}\left(\omega+\beta \Lambda_{1}^{-1}\left(\rho_{t-1}\right)+\alpha \frac{1}{m} \sum_{i=1}^{m} \Phi^{-1}\left(U_{1, t-i}\right) \Phi^{-1}\left(U_{2, t-i}\right)\right), \\
\Lambda_{1}(x) & =\frac{1-\exp (-x)}{1+\exp (-x)},
\end{aligned}
$$

where $\Lambda_{1}(\cdot)$ is a transformation function which holds the correlation parameter $\rho_{t}$ in the interval $(-1,1), \Phi(\cdot)$ is the standard normal $c d f$ and $m$ is an arbitrary window length. If the data is positively dependent, the inverse of marginal transforms of both variables will have the same sign. Thus, in case of positive dependence the parameter $\alpha$ should be positive.

For the non-Gaussian case Patton suggests modeling the tail dependence parameters $\left(\lambda^{U}\right.$ and $\lambda^{L}$ ) of the Symmetrized Joe-Clayton (SJC) copula, where $\lambda^{U}$ and $\lambda^{L}$ are stand-alone monotonic transformations to copula parameters ${ }^{2}$. In general, the model for the evolution of a dependence parameter (or tail dependence) of a copula is

$$
\theta_{t}=\Lambda_{2}\left(\omega+\beta \Lambda_{2}^{-1}\left(\theta_{t-1}\right)+\alpha \frac{1}{m} \sum_{j=0}^{m-1}\left|U_{1, t-j}-U_{2, t-j}\right|\right)
$$

where $\Lambda_{2}(x)$ is an appropriate transformation function to ensure the parameter always remains in its domain: $(1+\exp (-x))^{-1}$ for tail dependence, $\exp (x)$ for Clayton copula and $(\exp (x)+1)$ for Gumbel copula. In case of perfect positive dependence the forcing variable $\left|U_{1, t}-U_{2, t}\right|$ is close to zero, therefore the parameter $\alpha$ is expected to be negative.

Creal et al. (2008) developed a unifying framework named Generalized Autoregressive Score (GAS) for time series processes with time varying parameters. A scaled score vector is used as an updating mechanism for the observation driven part of a model. In general, the model GAS(p,q) for a time-varying parameter $f_{t}$ looks as follows

$$
f_{t}=\omega+\sum_{j=1}^{q} \beta_{j} f_{t-j}+\sum_{i=0}^{p-1} \alpha_{i} s_{t-i}
$$

where $s_{t}=S_{t-1} \cdot \nabla_{t}$ is the scaled score of the log-likelihood function of the model of interest. $\nabla_{t}$ is the first derivative of the log-likelihood with respect to the parameter, whereas $S_{t-1}$ is the scaling matrix, which is approximated by the inverse of Fisher information matrix.

\footnotetext{
${ }^{2}$ The Joe-Clayton copula is such a transformation of the Clayton copula that possesses upper and lower dependence and it is characterized by two parameters; the SJC allows for the special case of the symmetry in the dependence.
} 
The GAS $(1,1)$ model for the correlation coefficient of the Gaussian copula is

$$
\begin{aligned}
& f_{t}=\omega+\beta f_{t-1}+\alpha \frac{2\left(y_{t}-\rho_{t-1}-\rho_{t-1}\left(1+\rho_{t-1}^{2}\right)^{-1}\left(z_{t}-2\right)\right)}{\left(1-\rho_{t-1}^{2}\right)} \\
& \rho_{t}=\Lambda_{1}\left(f_{t}\right)
\end{aligned}
$$

where $y_{t}=\Phi^{-1}\left(U_{1 t}\right) \cdot \Phi^{-1}\left(U_{2 t}\right)$ and $z_{t}=\Phi^{-1}\left(U_{1 t}\right)^{2}+\Phi^{-1}\left(U_{2 t}\right)^{2}$.

Such a specification is more sensitive to the off-diagonal observations than the Patton model and the correlation parameter more rapidly adjusts to the decrease in dependence as illustrated nicely by Creal et al. (2008). The GAS model is also shown to be more sensitive to observations in the lower and upper tail.

This approach is also applicable to Archimedean copulas and, unlike Patton's model, it can be used for multivariate data. However, the problems with computing $s_{t}=\nabla_{t} \mathscr{I}_{t-1}^{-1}$ term might occur. A numerical approximation is suggested for obtaining the Fisher information matrix $\mathscr{I}_{t-1}=$ $E_{t-1}\left[\left(\nabla_{t}\right)^{2}\right]$. The conducted simulation study shows that GAS model provides an estimator, which is closer to the true parameter but has a higher variation.

A further paper dealing with dynamic copulas is Jondeau and Rockinger (2006), who model timevarying correlations for Gaussian and Student copulas in three different ways. Two of them, DCC correlations and regime-switching correlations, will be described in Sections 4.3.2 and 4.3.6. The third way can be seen as a discrete variation of the forcing equation by Patton (2006b). For this the unit square is split into a number of subsets $\mathscr{A}_{j}, j=1, \ldots, 16$. The choice of the subintervals can be chosen by the modeler and the authors suggest using 16 equally sized sub-squares over the grid 0 , $0.25,0.5,0.75,1$. Correlation then is given by

$$
\rho_{t}=\sum_{j=1}^{16} d_{j} \mathbf{I}\left[\left(U_{1 t-1}, U_{2 t-1}\right) \in \mathscr{A}_{j}\right],
$$

with $d_{j} \in[-1,1]$ and $\mathbf{I}$ the indicator function. Thus correlation at time $t$ is driven by the concordance of the observations at $t-1$.

Estimation of the observation driven models is based on the maximization of the copula loglikelihood as in (4.7), having the vector of parameters as an argument and treating the evolution function of $\theta_{t}$ as a constraint. 


\subsubsection{DCC copulas}

Engle (2002) proposed a multivariate GARCH model with dynamic conditional correlations (DCC), where the correlations are driven by the cross product of the lagged standardized residuals and an autoregressive term. Estimation is done, similarly as for copula models, by first estimating the GARCH parameters for the individual series and then estimating the parameters driving the correlation dynamics. This specification can easily be adapted to model the dynamics of copula parameters. Let $Y_{i t}=\Phi^{-1}\left(U_{i t}\right)$, where $\Phi$ denotes the $c d f$ of the standard normal distribution and $Y_{t}=\left(Y_{1 t}, Y_{2 t}\right)^{\prime}$. Then the DCC model specifies the correlation matrix $R_{t}$ as

$$
R_{t}=\operatorname{diag}\left\{Q_{t}\right\}^{-1 / 2} Q_{t} \operatorname{diag}\left\{Q_{t}\right\}^{-1 / 2}
$$

where $Q_{t}$ follows

$$
Q_{t}=\Omega(1-\alpha-\beta)+\alpha Y_{t-1} Y_{t-1}^{\prime}+\beta Q_{t-1}
$$

and $\Omega$ is the unconditional covariance matrix of $Y_{t-1}$. This specification ensures positive definiteness of the correlation matrix and that the correlation coefficient $\rho_{t}$, which is the off-diagonal element of $R_{t}$, lies in $[-1,1]$ at all times. Heinen and Valdesogo (2008) suggest how this approach can be extended to some non-elliptical copulas. They propose transforming the correlations into Kendall's tau through

$$
\tau_{t}^{K}=\frac{2}{\pi} \arcsin \left(\rho_{t}\right)
$$

Some copulas have a one-to-one relation between Kendall's tau and the dependence parameter $\theta$ and using this relationship the $\tau_{t}^{K}$ is mapped into $\theta_{t}$. As some copulas only allow for positive dependence, Heinen and Valdesogo (2008) overcome this potential problem by replacing the off-diagonal elements of $Q_{t}$ by $\max \left(0, q_{t}\right)$ to ensure that the copula parameter always remains in its domain. Thus, the negative dependence is treated by setting the corresponding copula to the independence copula. This can be seen as a potential drawback, but as the authors mention when the conditional correlation is below zero a large fraction of the time, models only allowing for positive dependence are likely to have bad fit and will not be considered to be appropriate very often. Another disadvantage of the DCC copula specification is that it is not obvious how to generalize it to copulas that have more than one parameter.

Estimation can be done by treating the copula parameter $\theta_{t}$ as an observable function of $\alpha, \beta$ 
and $\mathscr{F}_{t-1}$, the information at time $t-1$. The copula likelihood (4.7) is then maximized over the parameters $\alpha$ and $\beta$ that drive the dependence parameter.

\subsubsection{Stochastic autoregressive copulas (SCAR)}

Hafner and Manner (2008) suggest a time-varying copula model where dynamics of the copula parameter are not driven by the observations as in the DCC or the Patton model, but where the copula parameter is driven by an independent stochastic process. Formally, $\theta_{t}=\Lambda\left(\lambda_{t}\right)$, where $\Lambda: \mathbb{R} \rightarrow \Theta$ is an appropriate transformation to ensure that the copula parameter remains in its domain and whose functional form depends on the choice of copula. The underlying process $\left\{\lambda_{t}\right\}_{t=1}^{T}$, which is latent, is assumed to follow a Gaussian autoregressive process of order one,

$$
\lambda_{t}=\omega+\beta \lambda_{t-1}+\sigma_{\eta} \eta_{t}
$$

where $\eta_{t}$ is an i.i.d. $N(0,1)$ innovation and $|\beta|<1$ to ensure stationarity of $\lambda_{t}$. For the Frank and the Plackett copulas the transformation $\Lambda$ is simply $\Lambda(x)=x$, implying normality of the copula parameter, for the Clayton copula it is $\Lambda(x)=\exp (x)$, and for the Gumbel copula $\Lambda(x)=\exp (x)+1$, implying log-normality of $\theta_{t}$ for these two families. For the Gaussian and the Student copulas the inverse Fisher transform $\Lambda(x)=(\exp (2 x)-1) /(\exp (2 x)+1)$ is the most natural choice, since the Fisher transform is the variance stabilizing transformation for the correlation coefficient (van der Vaart 1998).

Estimation of the parameter vector $\left(\omega, \beta, \sigma_{\eta}\right)$ is not straightforward since the process $\left\{\lambda_{t}\right\}_{t=1}^{T}$ is unobservable. Hafner and Manner (2008) propose to integrate it out of the likelihood function of the copula. Denote $U_{1}=\left\{U_{1 t}\right\}_{t=1}^{T}, U_{2}=\left\{U_{2 t}\right\}_{t=1}^{T}, \lambda=\left\{\lambda_{t}\right\}_{t=1}^{T}$ and let $f\left(U_{1}, U_{2}, \lambda ; \omega, \beta, \sigma_{\eta}\right)$ be the joint density of the observable variables $\left(U_{1}, U_{2}\right)$ and the latent process $\left\{\lambda_{t}\right\}_{t=1}^{T}$. Then the likelihood function is given by

$$
\mathscr{L}\left(\omega, \beta, \sigma_{\eta} ; U_{1}, U_{2}\right)=\int f\left(U_{1}, U_{2}, \lambda ; \omega, \beta, \sigma_{\eta}\right) d \lambda
$$

Hafner and Manner (2008) discuss how the efficient importance sampler (EIS) by Liesenfeld and Richard (2003) and Richard and Zhang (2007) can be adapted to evaluate this T-dimensional integral by simulation. The simulated likelihood function can then be maximized over the parameter vector $\left(\omega, \beta, \sigma_{\eta}\right)$. As a byproduct one obtains a smoothed estimate $\hat{\lambda}_{t}$ of the underlying latent process and thus also a smoothed estimate $\hat{\theta}_{t}$ of the time-varying copula parameter. 


\subsubsection{Semiparametric dynamic copula (SDC)}

Hafner and Reznikova (2008) propose a semiparamteric approach to model the time-varying behavior of the dependence parameter of a copula treating $\theta$ as a smooth function of time. On the second stage of the estimation the log-likelihood function from (4.7) is locally weighted around location $\tau$

$$
L(\theta ; h, \tau)=\sum_{t=1}^{T} \log c\left(U_{1 t}, U_{2 t} ; \theta\right) \cdot K_{h}(t / T-\tau),
$$

where $K(\cdot)$ is a kernel function, $K_{h}(\cdot)=(1 / h) K(\cdot), h>0$ is a bandwidth and $\tau \in[0,1]$ is an appropriate grid. Then the locally estimated dependence parameter takes the form:

$$
\widehat{\theta}(\tau)=\underset{\theta}{\arg \max } L(\theta ; h, \tau) .
$$

In the case when $K(\cdot)$ is a symmetric function, the estimator can possess a considerable bias at the boundaries, which is a well known problem of kernel estimation techniques (see Simonoff (1996), Ch.3). A possible solution is to approximate $\theta$ by a higher order polynomial, e.g. by simply taking the local linear function

$$
\theta(t / T) \approx \theta(\tau)+\theta^{\prime}(\tau)\left(\frac{t}{T}-\tau\right)
$$

The important step prior to estimation of $\theta$ is the bandwidth selection. The MSE-optimal bandwidth is

$$
\widehat{h}=\underset{h}{\arg \min }\left\{\int \widehat{M S E}(x ; h) w(x) d x\right\},
$$

where $\widehat{M S E}(\tau ; h)=\widehat{\operatorname{bias}}^{2}(\tau ; h)+\widehat{\operatorname{var}}(\tau ; h)$ and $w(x)$ is any weight function. To obtain the estimators of the bias and variance one needs first to select the pilot bandwidth $h^{*}$, which is the minimum of the integrated Extended Residual Square Criterion (ERSC) of Fan, Farmen, and Gijbels (1998)

$$
\operatorname{ERSC}(\tau ; h)=J_{T}^{-2}(\tau) s_{T}(\tau)\left\{1+\frac{\|K\|^{2}}{n h}\right\}
$$

where $J_{T}(\tau)=\ell_{[\tau T]}^{\prime \prime}(\widehat{\theta}(\tau)), s_{T}(\tau)=\frac{\sum_{t=1}^{T}\left(\ell_{\tau T}^{\prime}\left(\theta^{*}(t / T)\right)\right)^{2} K_{h}(t / T-\tau)}{\sum_{t=1}^{T} K_{h}(t / T-\tau)}$ with $\ell_{t}(\theta)=\log c\left(U_{1 t}, U_{2 t} ; \theta\right)$ and $\theta^{*}(t / T)$ is estimated for the local quadratic function.

If $T$ is not equal to the number of grid subintervals, then the estimated $\theta(\tau)$ is extrapolated on $[1, T]$. Hafner and Reznikova (2008) also provide the asymptotic theory for the $\theta$ estimator. 


\subsubsection{Structural breaks}

Another possibility to allow for changing dependence over time is to test for a structural break in the copula parameter at a given point in time $t^{*}$ as suggested by Dias and Embrechts (2004). Let the distribution of $U_{t}=\left(U_{1 t}, U_{2 t}\right)^{\prime}$ be $C\left(U_{1 t}, U_{2 t}, \theta_{t}\right)$, where $t=1, \ldots, T$. Formally, the null hypothesis of no structural break in the copula parameter becomes

$$
H_{0}: \theta_{t}=\theta
$$

whereas the alternative hypothesis of the presence of a single structural break is formulated as:

$$
H_{1}: \theta_{t}=\left\{\begin{array}{cc}
\theta_{1} & 1 \leqslant t \leqslant t^{*} \\
\theta_{2} & t^{*}<t \leqslant T .
\end{array}\right.
$$

In the case of a known break-point $t^{*}$, the test statistics can be derived as a generalized likelihood ratio test. Let $L_{1}(\theta), L_{2}(\theta)$ and $L(\theta)$ be the log-likelihood functions of the copula using the first $t^{*}$ observations, the observations from $t^{*}+1$ to $T$ and all observations, respectively. Then the likelihood ratio statistic can be written as

$$
L R_{t^{*}}=2\left[L_{1}\left(\hat{\theta}_{1}\right)+L_{2}\left(\hat{\theta}_{2}\right)-L(\hat{\theta})\right]
$$

where a hat denotes the maximizer of the corresponding likelihood function. Note that $\hat{\theta}_{1}$ and $\hat{\theta}_{2}$ denote the estimates of $\theta$ before and after the break, whereas $\hat{\theta}$ is the estimate of $\theta$ using the full sample. For $t^{*}$ fixed this statistic follows a $\chi^{2}$ distribution with the number of degrees of freedom equal to the dimension of $\theta$. In the case of an unknown break date $t^{*}$, a procedure similar to the one proposed in Andrews (1993) can be applied. The test statistic proposed by Dias and Embrechts (2004) is the supremum of the sequence of statistics for known $t^{*}$

$$
Z_{T}=\max _{1 \leqslant t^{*}<T} L R_{t^{*}}
$$

and the asymptotic critical values of Andrews (1993) can be used.

Candelon and Manner (2007) have extended the procedure to additionally allow for a breakpoint in the parameters of the marginal distribution at a (possibly) different point in time and they propose a bootstrap procedure to obtain critical values of the test statistic. 


\subsubsection{Adaptive estimation method (LCP)}

Giacomini et al. (2009) propose to estimate the time-varying parameters of the copula adaptively by means of local parametric fitting. The main idea is that the varying copula parameter $\theta_{t}$ can be well approximated by a constant $\theta$ on an interval of homogeneity $I_{t}$. The crucial point is how to estimate the length of each interval $\forall t$. This distinguishes the model from the simple case of moving window estimator, as for this method the length of the window is determined by a data driven procedure.

The Local Change Point (LCP) method developed by Mercurio and Spokoiny (2004) determines the largest interval where the dependency parameter is invariant. The method tests the hypothesis of homogeneity for the interval $I_{t}=\left[t-m_{t}, t\right)$ with the right end-point $t$. As soon as the length of the interval $m_{t}$ is estimated, the parameter $\theta_{t}$ is approximated by a constant estimator $\widehat{\theta}_{\widehat{I}_{t}}$. The method is carried out in the counter direction for $t=T, \ldots, 1$.

The length of the interval of homogeneity $I_{t}$ is estimated as follows. First, a family of nested intervals is defined as $\mathscr{I}=\left\{I_{k}=\left[t-m_{k}, t\right), k=1,2, \ldots\right\}$, such that $m_{k+1}>m_{k}$. Then, within an interval $I_{k}$ a set of internal points $\mathscr{T}_{k} \subset I_{k}$ is selected. This set of points $\mathscr{T}_{k}$ is suspected to contain a break-point $t^{*}$. The procedure works as follows:

1. Test the hypothesis of homogeneity on $\mathscr{T}_{k} \subset I_{k}$. The null and the alternative hypothesis are similar to (4.24) and (4.25). As for the likelihood ratio test in (4.26), here the point $t^{*} \in \mathscr{T}_{k}$ divides the testing interval $I_{k}$ in two disjoint intervals $I_{1}$ and $I_{2}$. Thus, the likelihoods are calculated for $I_{k}, I_{1}$ and $I_{2}$ with the ML estimators $\widetilde{\theta}_{k}, \widetilde{\theta}_{1}$ and $\widetilde{\theta}_{2}$. The corresponding $Z_{I_{k}}$ statistics from (4.27) is then compared to the critical value. The hypothesis of homogeneity of $\theta$ is rejected when $Z_{I_{k}}$ exceeds the critical value.

2. If $H_{0}$ for $k$ is not rejected, then the next interval $I_{k+1}$ is tested for homogeneity.

3. If $H_{0}$ is rejected on $I_{k}$, then the interval of homogeneity is the last accepted interval $\widehat{I}_{t}=I_{k-1}$. If a large window is selected the estimate of dependence is not sensitive and reacts to changes in dependence with high delay. On the contrary, if a window is small, the estimate is quite unstable with high perturbation. This is also the case for the first observations, for which the window is forced to be small. The size of the window depends on the choice of the critical values and other parameters, described in Giacomini et al. (2009) and Mercurio and Spokoiny (2004). subsectionRegime switching copulas (RSC) A further way to specify a copula model in which both the degree and the type of dependence change over time is to allow for a number of states, each being characterized by a different copula. These copulas can be from the same family but allowing for different parameters. They 
may, however, also change their functional forms implying different states having entirely different dependence structures, a possibility we do not consider here, but that allows for interesting modeling of financial data. One may think of a model distinguishing tranquil and crisis time, the former being characterized by a Gaussian copula, whereas during the latter data is being generated by a copula allowing for lower tail dependence. To our knowledge the first to allow for regime switching in correlations is Pelletier (2006). Garcia and Tsafack (2008) and Chollete et al. (2009) have explicitly modeled copulas in a regime switching framework. Let $k_{t}$ be a latent random variable that takes on the value $k=1, \ldots, K$ when regime $k$ is the current state. Then

$$
\left(U_{1 t}, U_{2 t} \mid k_{t}=k\right) \sim C\left(U_{1 t}, U_{2 t} ; \theta_{k}\right)
$$

and $k_{t}$ is assumed to follow a Markov chain of order one with $\pi_{i j}$ the probability of moving to regime $j$ in period $t$ conditional on being in state $i$ at time $t-1$. Usually the number of states $K$ is taken to be equal to two or three. $K=2$ is the more common choice which we focus on in this study. Estimation can be done using the Expectation Maximization (EM) algorithm as outlined in Hamilton (1994) Ch. 22. Define the matrix of transition probabilities

$$
P=\left(\begin{array}{cc}
\pi_{11} & 1-\pi_{11} \\
1-\pi_{22} & \pi_{22}
\end{array}\right)
$$

and let $\hat{\xi}_{t \mid t}$ be a $(2 \times 1)$ vector containing the estimated probabilities of being in each state at time $t$ given the information at time $t$. Further $\hat{\xi}_{t \mid t-1}$ are the same estimated probabilities only using information until time $t-1$. Then the system is described by

$$
\begin{aligned}
& \hat{\xi}_{t \mid t}=\frac{\hat{\xi}_{t \mid t-1} \odot \eta_{t}}{\mathbf{1}^{\prime}\left(\hat{\xi}_{t \mid t-1} \odot \eta_{t}\right)}, \\
& \hat{\xi}_{t+1 \mid t}=P^{\prime} \hat{\xi}_{t \mid t}, \\
& \eta_{t}=\left(\begin{array}{c}
c_{1}\left(U_{1 t}, U_{2 t} ; \theta_{1}\right) \\
c_{2}\left(U_{1 t}, U_{2 t} ; \theta_{2}\right)
\end{array}\right),
\end{aligned}
$$

with 1 a vector of ones and $\odot$ the Hadamard product ${ }^{3}$. For a given starting value $\hat{\xi}_{1 \mid 0}$ and copula parameters $\theta_{1}, \theta_{2}$ and transition probabilities $\pi_{11}$ and $\pi_{22}$ one can iterate over (4.29) and (4.30) to

\footnotetext{
${ }^{3}$ The Hadamard product denotes element by element multiplication of two equally sized matrices.
} 
obtain the log-likelihood function of the copula

$$
L L_{C}\left(\theta_{1}, \theta_{2}, \pi_{11}, \pi_{22} ; U_{1 t}, U_{2 t}\right)=\sum_{t=1}^{T} \log \left(\mathbf{1}^{\prime}\left(\hat{\xi}_{t \mid t-1} \odot \eta_{t}\right)\right)
$$

Formulas to estimate the smoothed probabilities of being in each state at time $t, \hat{\xi}_{t \mid T}$, can be found in Hamilton (1994).

\subsubsection{Other approaches}

In this section we shortly review additional approaches for testing for and modeling time-varying copulas that have been proposed in the literature. However, we will skip most of the details for the sake of brevity.

\section{van den Goorbergh et al. (2005):}

In this paper time-varying copulas are used to price options with multiple underlying assets and it is found that the option prices implied by time-varying copulas are quite different from those using static copulas. The relation between the parameter of some one-parameter copulas and Kendall's tau is exploited to estimate the copula parameter by a moment type estimator. A time-varying measure of Kendall's tau then implies a time-varying copula parameter. It is assumed that dependence is driven by the volatility of the assets, which is reasonable as it is implied by factor models for asset pricing and this relation has been confirmed in a number of studies. Let $h_{i t}$ be the conditional variance of asset $i$ (e.g. the GARCH variance). Then $\tau_{t}$ is assumed to follow

$$
\tau_{t}=\gamma_{0}+\gamma_{1} \log \left\{\max \left(h_{1 t}, h_{2 t}\right)\right\}
$$

The parameters $\gamma_{0}$ and $\gamma_{1}$ are estimated by regressing a rolling window estimate of $\tau_{t}$ on a constant and the maximum of the logarithm of the maximum of the GARCH variances. The window size is chosen to be equal to about 40 days, although it is found that the results are robust to the choice of the window size.

The main difference of this approach to the ones presented so far is that the copula parameter is assumed to depend on the marginal distribution through the conditional variance, whereas all the other approaches assume that the copula parameter behaves independently of the parameters of the marginal distributions. 


\section{Guégan and Zhang (2009):}

The difference between this approach to the majority of the competing approaches is that the authors do not only test for a change in the relationship between the variables of interest, but also whether the copula remains the same and only the degree of dependence changes, or whether additionally also the type of copula changes at a given point in time ${ }^{4}$. The main idea is to compare a parametric copula to a nonparametric estimate of the copula density at $m$ distinct points in time using the goodness-of-fit tests by Fermanian (2005). By applying the test to a conditional copula one can check whether the copula family changes. When the copula family changes the authors suggest using a binary segmentation procedure to detect the change points and the type of copula on each sub-interval, otherwise they suggest using the structural break test by Dias and Embrechts (2004) to detect the change points of the copula parameters. For the details of the procedure and the test statistics we refer the interested reader to the original paper.

\section{Harvey (2009):}

A further technique worth mentioning is that of Harvey (2009), who treats the problem of changing copulas by noting that it is related to estimating time-varying quantiles. The method is nonparametric and very different to the other techniques described here. Busetti and Harvey (2008) build on the same methods to construct a formal test for changing dependence. A description of the approaches is beyond the scope of this chapter.

\subsection{Model selection and simulations}

In this section we study how to measure the goodness-of-fit for time-varying copulas, how to select the best fitting model and how well the competing specifications for time-varying dependence presented in the previous section are able to estimate the underlying dependence process.

\subsubsection{Specification testing}

Assume for a given time series of observations $\left(U_{1 t}, U_{2 t}\right), t=1, \ldots, T$ copula model $C_{i}$ has been estimated, where $i$ denotes a candidate parametric copula, and an estimate for the sequence of dependence parameters $\hat{\theta}_{i t}, t=1, \ldots, T$ has been obtained. The first thing we are interested in is which of the competing models $C_{i}$ fits the data at hand best. Even though the models are usually non-nested and standard likelihood ratio test cannot be performed a very simple and (as we shall

\footnotetext{
${ }^{4}$ One exception is the regime switching copula presented in Section 4.3.6.
} 
see) reliable way to select the best fitting model is to compare the value of the log-likelihood function $L L_{i}$. The model with the highest likelihood is considered to be the best fitting one ${ }^{5}$.

The model maximizing the $L L$ statistics, however, must not necessarily provide a satisfactory fit for the data being analyzed. Thus, for an estimate $\hat{\theta}_{i t}, t=1, \ldots, T$ the hypothesis of interest is whether the data has actually been generated by $C_{i}$. Let $C_{0}\left(U_{1 t}, U_{2 t}, \theta_{t}^{0}\right)$ be the true copula where $\theta_{t}^{0}$ denotes the true parameter at time $t$. Then formally the null hypothesis is

$$
H_{0}: C_{i}\left(U_{1 t}, U_{2 t}, \hat{\theta}_{i t}\right)=C_{0}\left(U_{1 t}, U_{2 t}, \theta_{t}^{0}\right)
$$

Note that this means that we are testing both the copula specification $C_{i}$ and the estimate of the latent dependence parameter of model $i, \hat{\theta}_{i t}$, and rejecting $H_{0}$ does not necessarily mean that the data was not generated by $C_{i}$. We test the hypothesis in (4.34) by testing whether the copula of $U_{1}$ given $U_{2}$ is uniformly distributed, which is an application of the Rosenblatt probability integral transformation. In our case this means

$$
\hat{z}_{t}=C_{i}\left(U_{1 t} \mid U_{2 t}, \hat{\theta}_{i t}\right)=\frac{\partial C_{i}\left(U_{1 t}, U_{2 t}, \hat{\theta}_{i t}\right)}{\partial U_{2 t}} \sim U(0,1)
$$

We test this hypothesis by applying the Anderson-Darling (Anderson and Darling (1952)) test, which is given by

$$
T_{A D}=\sup _{x} \frac{\sqrt{T}|\hat{\mathbb{F}}(x)-F(x)|}{\sqrt{F(x)(1-F(x))}},
$$

where $\hat{\mathbb{F}}(\cdot)$ denotes the empirical $c d f$ of $\hat{z}_{t}$ and $F(x)$ is the $U(0,1) c d f$. For this statistics tabulated critical values must be used. Contrary to applying the test in the static copula setting for the timevarying case we are actually not only testing the functional form, but, as mentioned above, also the quality of the estimate $\hat{\theta}_{i t}, t=1, \ldots, T$, which may cause size distortions and influence the power of the tests.

\subsubsection{Monte Carlo study}

The simulation setup is as follows. We randomly draw a sample $\left(U_{1 t}, U_{2 t}\right)_{t=1}^{T}$ from a Gaussian copula with time-varying correlation coefficient. The correlations follow three alternative processes, two of

\footnotetext{
${ }^{5}$ It is theoretically more sound to use the Akaike Information Criterion (AIC) to compare the fit of non-nested models, but since we only compare the fit within each specification for the time-variation, the number of parameters is always the same and hence it is equivalent to looking at the value of the log-likelihood function.
} 
Table 4.1: MSE for estimating the underlying correlation

\begin{tabular}{rrrrrrrr}
\hline \hline MSE & Const & DCC & PATT & SDC & LCP & SCAR & RSC \\
\hline Step & 0.092 & 0.016 & 0.053 & 0.007 & 0.017 & 0.008 & 0.004 \\
& $(0.002)$ & $(0.004)$ & $(0.005)$ & $(0.003)$ & $(0.005)$ & $(0.003)$ & $(0.003)$ \\
Sine & 0.082 & 0.021 & 0.048 & 0.006 & 0.047 & 0.010 & 0.020 \\
& $(0.002)$ & $(0.004)$ & $(0.005)$ & $(0.003)$ & $(0.007)$ & $(0.003)$ & $(0.005)$ \\
AR(1) & 0.076 & 0.040 & 0.052 & 0.035 & 0.063 & 0.025 & 0.036 \\
& $(0.022)$ & $(0.009)$ & $(0.011)$ & $(0.008)$ & $(0.014)$ & $(0.006)$ & $(0.010)$ \\
\hline \hline
\end{tabular}

Note: Table 4.1 reports the MSE for estimating the underlying correlation process for data that has been generated by Gaussian copulas with correlation following a Step, Sine and AR(1) processes. Monte Carlo standard errors are given in parenthesis. The sample size is 1000 and the number of Monte Carlo replications is equal to 1000 for Const, DCC, PATT and RSC, 250 for SDC and SCAR, and 100 for LCP.

which are deterministic and one is stochastic:

1. Step: $\rho_{t}=0.2+0.6 I_{t>500}$

2. Sine: $\rho_{t}=0.5+0.4 \cos (2 \pi t / 400)$

3. $\operatorname{AR}(1): \rho_{t}=\left(\exp \left(2 \lambda_{t}\right)-1\right) /\left(\exp \left(2 \lambda_{t}\right)+1\right)$ with $\lambda_{t}=0.02+0.97 \lambda_{t-1}+0.1 \varepsilon_{t}$,

where $\varepsilon_{t} \sim N(0,1)$. Note that the average correlation is 0.5 for each of the data generating processes. We decided to leave out the case of data generated by a model with constant correlation, but we note that the models seem to be able to deal well with the case of constant dependence. Some simulation results for this situation can be found in Hafner and Manner (2008) and Hafner and Reznikova (2008). For each artificial data set we estimate the Gaussian, Frank, Gumbel and Clayton copulas with the following method to allow for time variation: Constant, DCC (§4.3.2), PATT (§4.3.1), SDC (§4.3.4), LCP (§4.3.6), SCAR (§4.3.3), and RSC (§4.3.6). For each estimation technique and each model $\hat{\theta}_{i t}, t=1, \ldots, T$ and $L L_{i}$ is obtained ${ }^{6}$. The sample size is equal to $T=1000$, corresponding to 4 years of daily data, and the number of Monte Carlo replications is 1000 in general, although due to the extremely high computational complexity it was only 250 for the SCAR and SDC models, and 100 for the LCP specification.

In order to get an idea of how well the competing time-varying copula models introduced above are able to estimate the underlying dependence parameter $\theta_{t}$ at each point in time we compute the

\footnotetext{
${ }^{6}$ For the regime switching copula $\hat{\theta}_{i t}$ is computed as the smoothed probabilities of being in each of the two states times the parameter in that state.
} 
Table 4.2: Model selection by the log-likelihood statistic

\begin{tabular}{rrrrrrrr}
\hline \hline & \multicolumn{7}{c}{ Step } \\
Const & DCC & PATT & SDC & LCP & SCAR & RSC \\
Gaussian & 0.212 & 0.997 & 0.011 & 0.996 & 0.720 & 0.968 & 0.990 \\
Clayton & 0.008 & 0.001 & 0.001 & 0.000 & 0.000 & 0.020 & 0.001 \\
Frank & 0.697 & 0.000 & 0.824 & 0.000 & 0.160 & 0.008 & 0.000 \\
Gumbel & 0.083 & 0.002 & 0.164 & 0.004 & 0.120 & 0.004 & 0.009 \\
\hline & Const & DCC & PATT & SDC & LCP & SCAR & RSC \\
Gaussian & 0.212 & 0.981 & 0.007 & 1.000 & 0.350 & 1.000 & 0.999 \\
Clayton & 0.008 & 0.002 & 0.002 & 0.000 & 0.010 & 0.000 & 0.000 \\
Frank & 0.697 & 0.006 & 0.488 & 0.000 & 0.260 & 0.000 & 0.000 \\
Gumbel & 0.083 & 0.011 & 0.503 & 0.000 & 0.380 & 0.000 & 0.001 \\
\hline & & \multicolumn{7}{c}{ AR(1) } & & & \\
Gaussian & 0.318 & 0.925 & 0.327 & 0.956 & 0.190 & 0.962 & 0.991 \\
Clayton & 0.004 & 0.001 & 0.002 & 0.000 & 0.060 & 0.034 & 0.000 \\
Frank & 0.608 & 0.043 & 0.312 & 0.024 & 0.360 & 0.004 & 0.005 \\
Gumbel & 0.070 & 0.031 & 0.359 & 0.02 & 0.390 & 0.000 & 0.004 \\
\hline \hline
\end{tabular}

Note: Table 4.2 reports the fraction of times each estimated copula has the highest log-likelihood statistics for data that has been generated by Gaussian copulas with correlation following a Step, Sine and AR(1) processes. Monte Carlo standard errors are given in parenthesis. The sample size is 1000 and the number of Monte Carlo replications is equal to 1000 for Const, DCC, PATT and RSC, 250 for SDC and SCAR, and 100 for LCP.

mean square distance between the true dependence parameter and its estimate

$$
M S E=\frac{1}{K} \sum_{k=1}^{K} \frac{1}{T} \sum_{t=1}^{T}\left(\hat{\theta}_{t}^{k}-\theta_{t}^{0 k}\right)^{2},
$$

where $K$ is the number of Monte Carlo replications, and $\theta_{t}^{0 k}$ and $\hat{\theta}_{t}^{k}$ denote the true and estimated dependence paths at replication $k$, respectively. Table 4.1 reports the average $M S E$ between the true and the estimated correlation processes for the Gaussian copula for the static and each of the timevarying copula specifications and for the different correlation dynamics. As expected, all models lead to substantial improvements over the constant copula model. However, the RSC, SCAR and SDC models are superior to the competing ones in all cases, the RSC being better for the Step correlation, SCAR for the AR(1) correlation and the SDC for the Sine correlation, as to be expected. The DCC performs worse than all three, but better than both PATT and LCP. The latter specification naturally does not do too well for the Sine and AR(1) correlations as the assumption of intervals of 
Table 4.3: Size and power of the Anderson-Darling test based on the probability integral transform

\begin{tabular}{|c|c|c|c|c|c|c|c|}
\hline \multicolumn{8}{|c|}{ Step } \\
\hline & Const & DCC & PATT & SDC & LCP & SCAR & RSC \\
\hline Gaussian & $\begin{array}{l}0.352 \\
(0.015)\end{array}$ & $\begin{array}{l}0.058 \\
(0.007)\end{array}$ & $\begin{array}{l}0.254 \\
(0.014)\end{array}$ & $\begin{array}{l}0.056 \\
(0.007)\end{array}$ & $\begin{array}{l}0.040 \\
(0.006)\end{array}$ & & $\begin{array}{l}0.048 \\
(0.007)\end{array}$ \\
\hline Clayton & $\begin{array}{l}0.643 \\
(0.015)\end{array}$ & $\begin{array}{l}0.864 \\
(0.011)\end{array}$ & $\begin{array}{l}0.619 \\
(0.015)\end{array}$ & $\begin{array}{l}0.600 \\
(0.015)\end{array}$ & $\begin{array}{l}0.480 \\
(0.016)\end{array}$ & $\begin{array}{l}0.737 \\
(0.014)\end{array}$ & $\begin{array}{l}0.838 \\
(0.012)\end{array}$ \\
\hline Frank & $\begin{array}{l}0.051 \\
(0.007)\end{array}$ & $\begin{array}{l}0.207 \\
(0.013)\end{array}$ & $\begin{array}{l}0.254 \\
(0.014)\end{array}$ & $\begin{array}{l}0.268 \\
(0.014)\end{array}$ & $\begin{array}{l}0.190 \\
(0.012)\end{array}$ & $\begin{array}{l}0.267 \\
(0.014)\end{array}$ & $\begin{array}{l}0.062 \\
(0.008)\end{array}$ \\
\hline Gumbel & $\begin{array}{l}0.539 \\
(0.016)\end{array}$ & $\begin{array}{l}0.621 \\
(0.015)\end{array}$ & $\begin{array}{l}0.584 \\
(0.016)\end{array}$ & $\begin{array}{l}0.564 \\
(0.016)\end{array}$ & $\begin{array}{l}0.420 \\
(0.016)\end{array}$ & & $\begin{array}{l}0.571 \\
(0.016)\end{array}$ \\
\hline \multicolumn{8}{|c|}{ Sine } \\
\hline & Const & DCC & PATT & SDC & LCP & SCAR & RSC \\
\hline Gaussian & $\begin{array}{l}0.352 \\
(0.015)\end{array}$ & $\begin{array}{l}0.129 \\
(0.011)\end{array}$ & $\begin{array}{l}0.324 \\
(0.015)\end{array}$ & $\begin{array}{l}0.068 \\
(0.008)\end{array}$ & $\begin{array}{l}0.260 \\
(0.014)\end{array}$ & & $\begin{array}{l}0.041 \\
(0.006)\end{array}$ \\
\hline Clayton & $\begin{array}{l}0.643 \\
(0.015)\end{array}$ & $\begin{array}{l}0.898 \\
(0.010)\end{array}$ & $\begin{array}{l}0.635 \\
(0.015)\end{array}$ & $\begin{array}{l}0.640 \\
(0.015)\end{array}$ & $\begin{array}{l}0.770 \\
(0.013)\end{array}$ & $\begin{array}{l}0.790 \\
(0.013)\end{array}$ & $\begin{array}{l}0.762 \\
(0.013)\end{array}$ \\
\hline Frank & $\begin{array}{l}0.051 \\
(0.007)\end{array}$ & $\begin{array}{l}0.142 \\
(0.011)\end{array}$ & $\begin{array}{l}0.134 \\
(0.011)\end{array}$ & $\begin{array}{l}0.212 \\
(0.013)\end{array}$ & $\begin{array}{l}0.110 \\
(0.010)\end{array}$ & & $\begin{array}{l}0.130 \\
(0.011)\end{array}$ \\
\hline Gumbel & $\begin{array}{l}0.539 \\
(0.016)\end{array}$ & $\begin{array}{l}0.625 \\
(0.015)\end{array}$ & $\begin{array}{l}0.561 \\
(0.016)\end{array}$ & $\begin{array}{l}0.552 \\
(0.016)\end{array}$ & $\begin{array}{l}0.520 \\
(0.016)\end{array}$ & & $\begin{array}{l}0.595 \\
(0.016)\end{array}$ \\
\hline \multicolumn{8}{|c|}{$\operatorname{AR}(1)$} \\
\hline & Const & DCC & PATT & SDC & LCP & SCAR & RSC \\
\hline Gaussian & $\begin{array}{l}0.291 \\
(0.014)\end{array}$ & $\begin{array}{l}0.160 \\
(0.012)\end{array}$ & $\begin{array}{l}0.249 \\
(0.014)\end{array}$ & $\begin{array}{l}0.100 \\
(0.019)\end{array}$ & $\begin{array}{l}0.370 \\
(0.048)\end{array}$ & $\begin{array}{l}0.076 \\
(0.017)\end{array}$ & $\begin{array}{l}0.054 \\
(0.007)\end{array}$ \\
\hline Clayton & $\begin{array}{l}0.656 \\
(0.015)\end{array}$ & $\begin{array}{l}0.897 \\
(0.010)\end{array}$ & $\begin{array}{l}0.661 \\
(0.015)\end{array}$ & $\begin{array}{c}0.708 \\
(0.014)\end{array}$ & $\begin{array}{l}0.730 \\
(0.014)\end{array}$ & $\begin{array}{l}0.810 \\
(0.012)\end{array}$ & $\begin{array}{l}0.858 \\
(0.011)\end{array}$ \\
\hline Frank & $\begin{array}{l}0.078 \\
(0.008)\end{array}$ & $\begin{array}{l}0.118 \\
(0.010)\end{array}$ & $\begin{array}{l}0.126 \\
(0.010)\end{array}$ & $\begin{array}{l}0.216 \\
(0.013)\end{array}$ & $\begin{array}{l}0.080 \\
(0.009)\end{array}$ & $\begin{array}{l}0.397 \\
(0.015)\end{array}$ & $\begin{array}{l}0.134 \\
(0.011)\end{array}$ \\
\hline $\mathrm{Gu}$ & $\begin{array}{l}0.555 \\
(0.016)\end{array}$ & $\begin{array}{l}0.652 \\
(0.015)\end{array}$ & $\begin{array}{l}0.605 \\
(0.015)\end{array}$ & $\begin{array}{l}0.636 \\
(0.015)\end{array}$ & $\begin{array}{l}0.590 \\
(0.016)\end{array}$ & $\begin{array}{l}0.741 \\
(0.014)\end{array}$ & $\begin{array}{c}0.668 \\
(0.015)\end{array}$ \\
\hline
\end{tabular}

Note: Table 4.3 reports the rejection frequency of the null hypothesis of correct copula specification using the Anderson-Darling test based on the probability integral transform at a $5 \%$ nominal level. Data has been generated by Gaussian copulas with correlation following a Step, Sine and AR(1) processes. Monte Carlo standard errors are given in parenthesis. The sample size is 1000 and the number of Monte Carlo replications is equal to 1000 for Const, DCC, PATT and RSC, 250 for SDC and SCAR, and 100 for LCP.

homogeneity is violated. Surprisingly, although the performance for the Step correlation is acceptable the MSEs are still higher than those of the DCC, SDC and SCAR models. This is puzzling insofar as this DGP should strongly favor the nature of the LCP procedure.

The fraction of times each copula is the selected as the best fitting one in terms of the highest $L L$ statistics can be found in Table 4.2. One has to keep in mind that the comparison of different copulas using the $L L$ statistic is only possible within the same specification for the time-variation, but cannot generally be used to compare different models for the dynamics in dependence. When ignoring the time-variation of dependence the Frank copula is chosen quite often. This suggests that the unconditional copula corresponding to a time-varying Gaussian copula is closer to the static 
Frank than to the Gaussian copula. When using the RSC, DCC, SDC and the SCAR models the $L L$ statistics turns out to be a very reliable model selection criterion. It does, however, become quite unreliable for both PATT and LCP, although for the latter the results for the step correlation are still acceptable.

The size and the power for the AD test at a 5\% nominal level are reported in Table 4.3. Monte Carlo standard errors are included, since for different models a different number of Monte Carlo replication was chosen. In terms of size the RSC model performs best closely followed by the SCAR model, which is slightly oversized for all cases. The SDC has higher size distortions, but still does quite well and the other models are all severely oversized with the exception of the DCC and the LCP models for the Step correlation. The power of the tests, which is not corrected for the size distortions, is best for the RSC, DCC and the SCAR models, although the SDC also has good power properties. All models have problems rejecting the Frank copula and in some cases the power against the Frank copula is even below the size. This is probably due to the fact that the Frank copula is quite similar to the Gaussian copula having no tail dependence and a symmetric dependence structure. The power against the asymmetric copulas looks better for all models.

Overall, we can conclude the RSC, SDC and SCAR specifications for time-varying copulas are superior to the competing specifications. These models do not only perform very well for the DGPs that clearly favor the models, namely Step for RSC, AR(1) for the SCAR and Sine for SDC, but also for the other DGP's. This shows the flexibility of these approaches. For the SDC it is due to the non-parametric nature of the parameter changes and the local estimation of the model. The SCAR model most likely performs well due to the high flexibility allowed for by including a random error term in the dependence process and the fact that the importance sampler exploited for its estimation makes efficient use of the information contained in the data. The usefulness and flexibility of the regime switching approach has already been shown for many other models and it seems to work equally well for copulas. Still, the DCC model also shows a rather good performance having the big advantage that it is easy to implement and that it does not require heavy computations, which in fact is also the case for the RSC.

Which model to use depends on the assumptions one is willing to make on the time-evolution of the dependence parameter, SDC being more suitable for smoothly changing processes, whereas the DCC and SCAR models are more appropriate for autoregressive correlations and regimes switching naturally applying when one believes in different states of the world. The simulation result showed that even for misspecified correlation dynamics these models perform well. Still, formal techniques to 
decide which method provides the best fit on a given data set need to be developed. From a practical point of view the choice of the model is also a matter of taste and software availability.

Note that although we only considered the Gaussian copula as the data generating process unreported simulations suggest that our findings continue to hold when the data is generated by different copulas.

\subsection{Empirical illustration}

For the empirical example we consider two data sets. The first data set are daily returns of the exchange rates of Yen-USD and Euro-USD. It contains 1564 observations from 31 December 1999 till 30 December 2005. The second data set are weekly returns of Morgan Stanley Capital International (MSCI) indexes of Korea and Singapore (in US Dollars) with 1039 observations from 10 May 1989 till 29 April 2009. With these examples we want to check the ability of the copula models to describe both data in tranquil and crisis times, and also to find out how much information is hidden in volatility vs. dependence.

The log-returns of Yen and Euro do not show any unusual behavior due to the selected observation period, with the skewness $(-0.06$ and -0.08$)$ and kurtosis $(3.61$ and 4.27$)$, respectively. The logreturns of Korea and Singapore MSCI indexes, on the other hand, show vivid evidence of the clusters of volatility (Dec'97 and Nov'08). The descriptive statistics also suggest that the observations should be filtered: both series posses negative skewness $(-0.48$ and -0.40$)$ and large kurtosis (9.64 and 5.87) respectively. The Jarque-Bera test for normality clearly rejects the null hypothesis for all series.

At the first stage of estimation of the models we model the marginal distributions of the data. We use $\operatorname{AR}(\mathrm{p})-\operatorname{GARCH}(1,1)$ models with Student-t error terms to correct the log-returns for the presence of autocorrelation and conditional heteroscedasticity. The number of lags $p$ of the AR(p) model is selected by Bayesian information criterion (BIC). Thus, the model for the log-returns $X_{i t}$ looks as follows:

$$
\begin{aligned}
X_{i t} & =\alpha_{i 0}+\sum_{j=1}^{p} \alpha_{i p} X_{i, t-j}+\varepsilon_{i t} \\
\varepsilon_{i t} & =\sqrt{h_{i t}} z_{i t} \\
h_{i t} & =\omega_{i}+\alpha_{i} \varepsilon_{i, t-1}^{2}+\beta_{i} h_{i, t-1},
\end{aligned}
$$

where $i$ is the index of the analyzed data series and $z_{i t}$ are standard-t distributed with $\nu_{i}$ degrease 
Table 4.4: First stage estimators: $\operatorname{AR}(\mathrm{p})-\mathrm{GARCH}(1,1)$ model

\begin{tabular}{|c|c|c|c|c|c|}
\hline & \multirow{2}{*}{$\begin{array}{l}\mathbf{A R}(\mathbf{p}) \\
\alpha_{0}, \alpha_{1}, \ldots \alpha_{p}\end{array}$} & \multicolumn{3}{|c|}{$\overline{\operatorname{GARCH}(\mathbf{1}, \mathbf{1})}$} & \multirow{2}{*}{$\begin{array}{c}\text { d.o.f. } \\
\nu\end{array}$} \\
\hline & & $\omega$ & $\alpha$ & $\beta$ & \\
\hline Euro & $\begin{array}{cc}-9.7 E-05, & -0.06 \\
(1.7 E-04) & (0.03)\end{array}$ & $\begin{array}{l}3.5 E-07 \\
(1.3 E-07)\end{array}$ & $\begin{array}{l}0.02 \\
(0.01)\end{array}$ & $\begin{array}{l}0.97 \\
(0.01)\end{array}$ & $\begin{array}{l}28.83 \\
(12.03)\end{array}$ \\
\hline Yen & $\begin{array}{cc}9.8 E-05, & -0.04 \\
(1.5 E-04) & (0.03)\end{array}$ & $\begin{array}{r}5.3 E-07 \\
(1.5 E-07)\end{array}$ & $\begin{array}{l}0.02 \\
(0.01)\end{array}$ & $\begin{array}{l}0.96 \\
(0.01)\end{array}$ & $\begin{array}{l}7.11 \\
(1.15)\end{array}$ \\
\hline Singapore & $\begin{array}{ll}5.3 E-04, & 0.06 \\
(9.9 E-04) & (0.03)\end{array}$ & $\begin{array}{l}1.6 E-05 \\
(7.9 E-06)\end{array}$ & $\begin{array}{l}0.11 \\
(0.03)\end{array}$ & $\begin{array}{l}0.88 \\
(0.03)\end{array}$ & $\begin{array}{l}7.48 \\
(1.58)\end{array}$ \\
\hline Korea & $\begin{array}{ccc}-4.8 E-02, & 0.03, & 0.13 \\
(3.1 E-02) & (0.03) & (0.03) \\
\end{array}$ & $\begin{array}{c}5.9 E-05 \\
(2.3 E-05)\end{array}$ & $\begin{array}{l}0.12 \\
(0.03) \\
\end{array}$ & $\begin{array}{l}0.86 \\
(0.03) \\
\end{array}$ & $\begin{array}{c}10.60 \\
(3.26)\end{array}$ \\
\hline
\end{tabular}

Note: Table 4.4 reports the estimated parameters and standard errors of the AR(p)GARCH(1,1)-t model for the log-returns of exchange rates Euro-USD and Yen-USD (daily observations, Dec'99 - Dec'05) and MSCI indexes of Singapore and Korea (weekly observations, May'89 - Apr'09).

of freedom. The first stage estimators are given in Table 4.4. The adequacy of the estimated models is tested by applying the Ljung-Box test on the estimated residuals.

Thus, we estimate $\widehat{z}_{i t}=\varepsilon_{i t} / \sqrt{\widehat{h}_{i t}}$, where $\widehat{z}_{i t} \sqrt{\frac{\nu_{i}}{\nu_{i}-2}}$ follows a Student-t distribution with $\nu_{i}$ degrees of freedom.

\subsubsection{Copula model for exchange rates of Euro-USD and Yen-USD}

Next we estimate the dependence structure between Euro-USD and Yen-USD with six types of copulas: two symmetric with no tail dependency (Gaussian, Frank), two with upper tail dependency (Gumbel, rotated Clayton), and two with lower tail dependency (Clayton, rotated Gumbel). These copulas and their properties are reviewed in the appendix. The models for the time evolution of the parameter are Constant, DCC, PATT, SCDM, LCP, SCAR and RSC discussed in previous sections. Table 4.5(a) reports the log-likelihoods of the estimated models. For each model the best fitting type of copula in terms of the likelihood is marked out in bold. As it is seen from the table the likelihoods of Constant, PATT and LCP models favor Frank copula, whereas DCC, SDC, SCAR and RSC models point to Gaussian copula. However, the log-likelihoods for the Frank copula are virtually identical in latter cases. Taking into account the finding of the Monte Carlo study that the DCC, SDC, SCAR and RSC models are more reliable when selecting the best fitting copula using the log-likelihood either the Frank or the Gaussian copula could be selected. Recall that in general it is not possible to compare the fit across different specification by looking at the log-likelihood, as not all models have the same number of parameters. However, the fit of the DCC, PATT and SCAR models may in fact be compared, because they do have the same number of parameters. 
Figure 4.1: Time-varying dependence for exchange rate returns
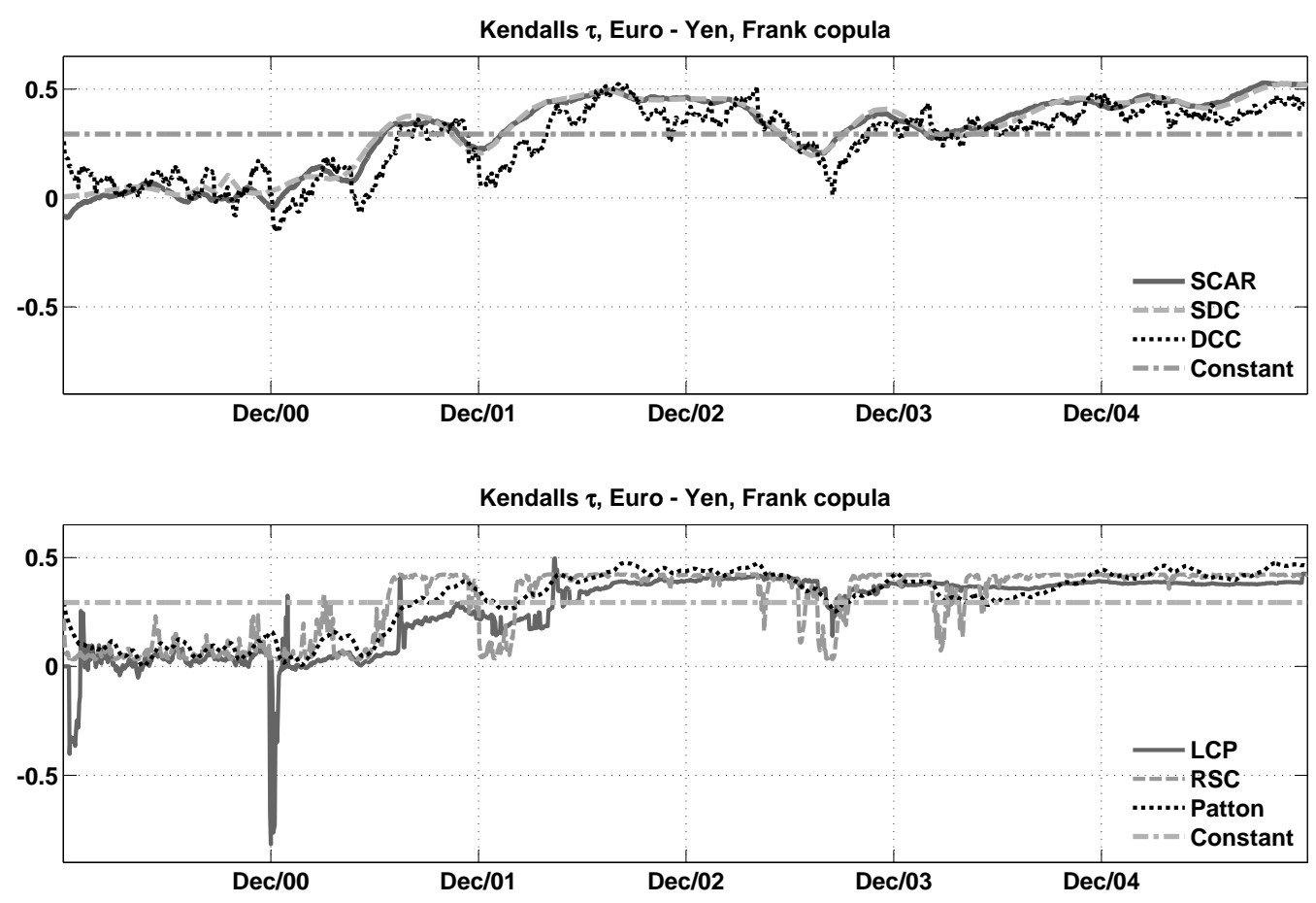

Note: Estimated dependence for the pair of exchange rates Euro-USD and Yen-USD. Frank copula. Dependence paths are transformed to Kendall's tau. Daily observations, Dec'99 - Dec'05.

The goodness-of-fit of the estimated models is then checked with Anderson-Darling (AD) test of correct copula specification, described in Section 4.4. The p-values of the test are presented in Table 4.5(b). The Frank copula passed the test for all estimated specifications, whereas all the other copulas are rejected. Taken as a whole, these findings strongly favor the Frank copula as the best fitting copula.

Figure 4.1 presents the dependence paths, estimated from Frank copula and transformed to Kendall's tau for the sake of comparison. The estimated paths of SDC and SCAR models are very close. Dependence estimated with Patton, DCC and RSC models show similar behavior as SDC and SCAR, but shifted to the right. This can be explained by the fact that the SDC and SCAR take into account the information of the full sample to estimate dependence at time $t$, whereas the other specifications only rely on past information. Finally, the erratic behavior of the dependence path estimated for LCP in 2001 suggests the presence of a sudden change. Indeed, the data seem to be independent until January 2001 and then the dependence grows considerably. This corresponds to 
Table 4.5: Model selection for Euro-USD and Yen-USD data

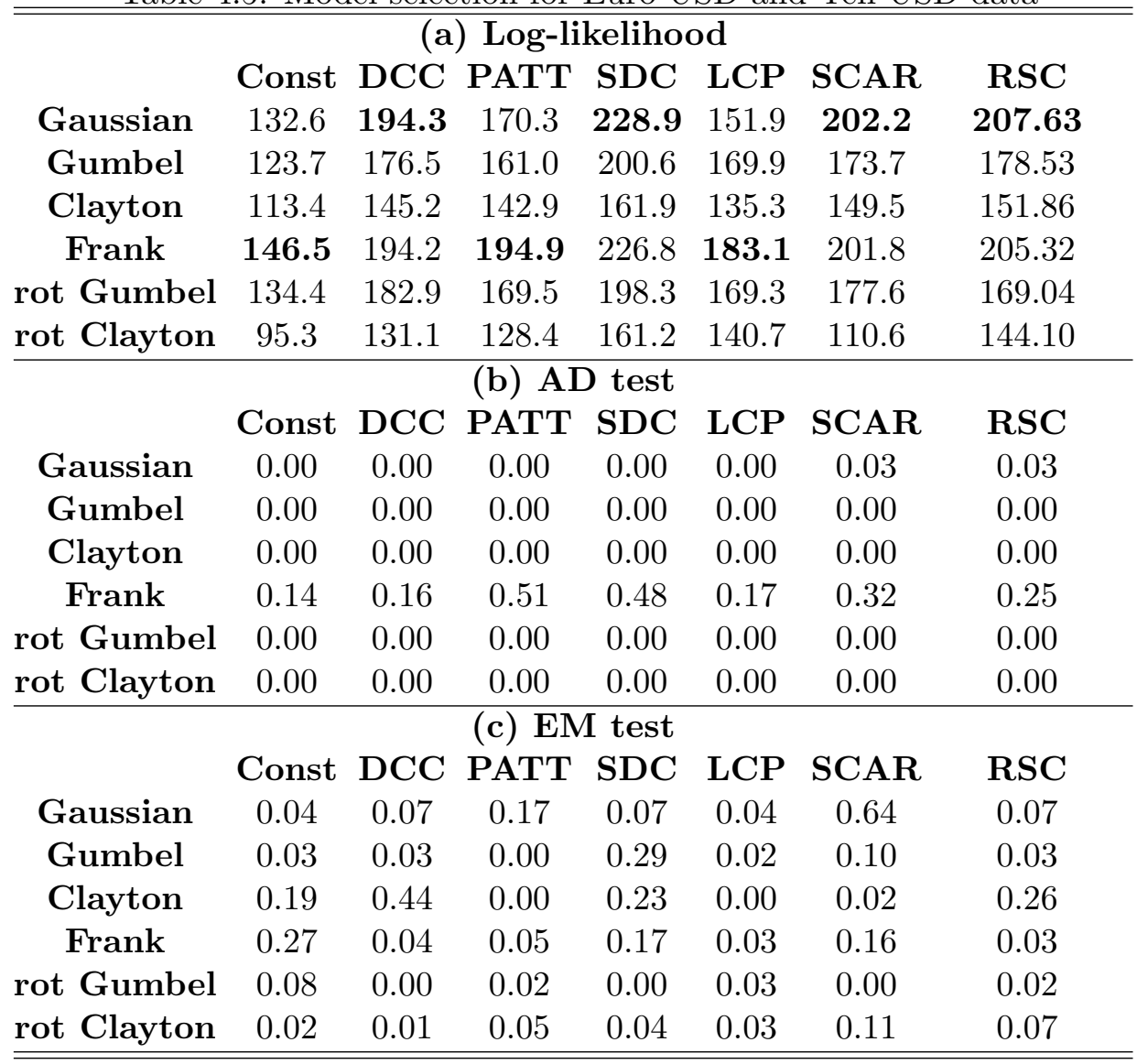

Note: Table 4.5 reports the log-likelihood (a), the p-values of the Anderson-Darling test for correct copula specification (b) and the p-values of the Engle-Manganelli test for the correct specification of the Value-at-Risk (c). The data are log-returns of the exchange rates Euro-USD and Yen-USD (daily observations, Dec'99 - Dec'05).

the expectations of the introduction of the Euro in January 2002.

The last measure that we use to test the adequacy of the estimated models is the Value-at-Risk (VaR) of an equally weighted portfolio. $\operatorname{VaR}_{t}(\alpha)$ is the $\alpha$-quantile of the conditional distribution of portfolio returns at time $t$, which can be obtained by simulation. Table 4.5(c) reports the results of the Dynamic Quantile (DQ) test of Engle and Manganelli (2004). The null hypothesis of the DQ test states that the model is correctly specified and that VaR is not under or over-estimated. The test is based on $F$ statistics and tests $H_{0}: \delta_{0}=\delta_{1}=\ldots=\delta_{6}=0$ for the regression:

$$
h i t_{t}^{\alpha}-\alpha=\delta_{0}+\delta_{1} h i t_{t-1}^{\alpha}+\ldots+\delta_{5} h i t_{t-5}^{\alpha}+\delta_{6} \operatorname{VaR}_{t}(\alpha)+\nu_{t}
$$

where $h_{t}^{\alpha}=\mathbb{I}\left(X_{t} \leqslant \operatorname{VaR}_{t}(\alpha)\right)$ and $X_{t}$ is the return of the portfolio. The results of the DQ test are shown in Table 4.5(c). For Gaussian and Frank copulas the estimated VaR has no autocorrelation 
in the hits for five and four out of seven models, respectively. For other types of copulas the Pvalues are in general close to zero. Thus, we can conclude that for this data example not only the properly estimated volatilities of the marginals matters, but also the dynamics of the joint dependence structure of the assets. However, as it will be shown in the next section, DQ test for VaR as a models' goodness-of-fit criterion should be used with care.

\subsubsection{Copula model for MSCI indexes of Korea and Singapore}

In the second application we consider weekly observations of the MSCI indexes of Korea and Singapore. As in the example above we estimate time-varying copula specifications for the same types of copulas. The results of the evaluated log-likelihoods can be found in Table 4.6(a). The log-likelihoods unambiguously point to the Gaussian copula as the best fitting copula type. As for the second best choice, for all seven models it is a rotated Gumbel copula. This provides some evidence of lower tail dependence, which is not a surprise given a financial crisis occurred in 1997 and stock market returns tend to have more dependence for losses than for gains.

The AD test results are reported in Table 4.6(b). The test rejects only Gumbel and rotated Clayton copulas, but approves all the other types. Given that it produces the highest log-likelihood statistic and that it is not rejected by the $\mathrm{AD}$ test the Gaussian copula seems to be the best fitting model, although one may argue in favor of the rotated Gumbel copula. The transforms to Kendall's tau of the dependence paths based on Gaussian copula are shown in Figure 4.2. The estimated paths of dependence for the SDC and SCAR models are very close and look quite smooth. The correlation estimated from DCC model is also very close to SDC and SCAR with some deviations. The dependence estimated from Patton's model is this time noisier than of DCC model and compared to the SDC/SCAR models lies closer to the unconditional dependence parameter throughout the sample. The RSC estimator vividly shows the periods of constancy of the dependence. However, the main shift in the dependence for this model falls on the year 2000. The dependence path estimated from the LCP model deviates a lot from the other models when the dependence increases due to the Asian crisis in 1997. Note that this increase in dependence provides evidence for financial contagion as studied using copulas in Rodriguez (2007) and Candelon and Manner (2007).

Finally, Table $4.6(\mathrm{c})$ provides the DQ test results. For this data example the DQ test approved the estimated VaR for almost all types of models and copulas. Such a result shows us that most of the risk information is hidden in the volatilities of the individual data series and less in the joint dependence structure. Thus, though it is demonstrated that the associated countries tend to be more 
Figure 4.2: Time-varying dependence for stock market returns
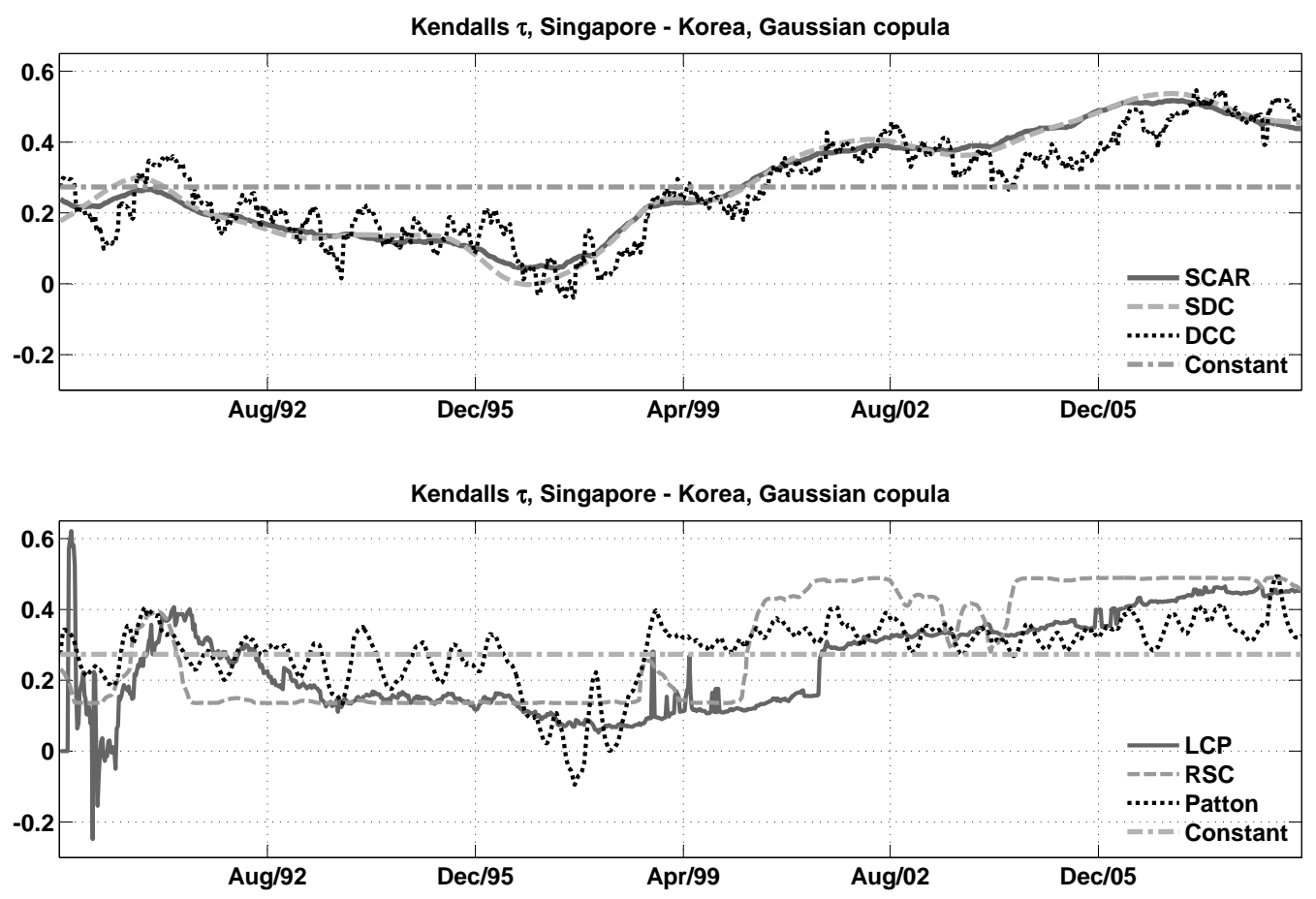

Note: Estimated dependence for the pair of MSCI indexes of Singapore and Korea. Gaussian copula. Dependence paths are transformed to Kendall's tau. Weekly observations, May'89 - Apr'09.

dependent during the crisis period and even after, the risk hidden in the dependence structure is not always relevant. Hence, DQ test is not a bona fide goodness-of-fit measure, but just an auxiliary method.

\subsection{Conclusions}

In this chapter we have provided a survey over existing copula models allowing for time-varying dependencies that have been proposed in recent years. Correctly modeling the dependence between financial assets plays a crucial role for measuring risks and pricing derivatives and since there is strong evidence that dependencies change over time, appropriately modeling and measuring these changes is not only interesting for its own sake, but also has important economic implications.

The different time-varying copula models we reviewed rely on different assumptions about the way dependence may change over time ranging from structural breaks in dependence, the existence of different dependence regimes, smooth changes or copula parameters behaving like an independent 
Table 4.6: Model selection for Singapore-Korea MSCI indexes

\begin{tabular}{|c|c|c|c|c|c|c|c|}
\hline \multicolumn{8}{|c|}{ (a) Log-likelihood } \\
\hline & Const & DCC & PATT & SDC & LCP & SCAR & RSC \\
\hline Gaussian & 98.5 & 134.6 & 120.4 & 149.1 & 117.7 & 133.9 & 127.39 \\
\hline Gumbel & 88.9 & 121.9 & 109.7 & 133.5 & 106.6 & 118.6 & 117.65 \\
\hline Clayton & 81.7 & 109.4 & 105.6 & 128.2 & 95.9 & 114.1 & 109.43 \\
\hline Frank & 87.9 & 117.0 & 106.1 & 130.6 & 101.8 & 116.2 & 106.31 \\
\hline rot Gumbel & 93.4 & 125.6 & 115.8 & 139.7 & 112.2 & 122.2 & 122.88 \\
\hline rot Clayton & 71.1 & 94.4 & 89.9 & 110.4 & 83.6 & 92.7 & 90.94 \\
\hline \multicolumn{8}{|c|}{ (b) AD test } \\
\hline & Const & $\mathrm{DCC}$ & PATT & SDC & LCP & SCAR & RSC \\
\hline Gaussian & 0.17 & 0.30 & 0.30 & 0.41 & 0.27 & 0.38 & 0.19 \\
\hline Gumbel & 0.02 & 0.00 & 0.01 & 0.01 & 0.01 & 0.00 & 0.00 \\
\hline Clayton & 0.75 & 0.04 & 0.49 & 0.15 & 0.60 & 0.14 & 0.15 \\
\hline Frank & 0.21 & 0.05 & 0.10 & 0.05 & 0.08 & 0.06 & 0.11 \\
\hline rot Gumbel & 0.34 & 0.13 & 0.59 & 0.14 & 0.53 & 0.17 & 0.51 \\
\hline rot Clayton & 0.03 & 0.00 & 0.01 & 0.00 & 0.02 & 0.00 & 0.00 \\
\hline \multicolumn{8}{|c|}{ (c) EM test } \\
\hline & Const & $\mathrm{DCC}$ & PATT & SDC & LCP & SCAR & RSC \\
\hline Gaussian & 0.76 & 0.45 & 0.14 & 0.14 & 0.14 & 0.79 & 0.86 \\
\hline Gumbel & 0.45 & 0.24 & 0.66 & 0.66 & 0.66 & 0.83 & 0.24 \\
\hline Clayton & 0.32 & 0.55 & 0.39 & 0.39 & 0.39 & 0.34 & 0.44 \\
\hline Frank & 0.01 & 0.11 & 0.17 & 0.17 & 0.17 & 0.49 & 0.91 \\
\hline rot Gumbel & 0.26 & 0.66 & 0.71 & 0.71 & 0.71 & 0.65 & 0.47 \\
\hline rot Clayton & 0.03 & 0.90 & 0.09 & 0.09 & 0.09 & 0.84 & 0.47 \\
\hline
\end{tabular}

Note: Table 4.6 reports the log-likelihood (a), the p-values of the Anderson-Darling test for correct copula specification (b) and the p-values of the Engle-Manganelli test for the correct specification of the Value-at-Risk (c). The data are log-returns of the MSCI indexes of Singapore and Korea (weekly observations, May'89 - Apr'09).

stochastic process. Since one cannot directly observe the dependence parameter and hence no $a$ priori type of dynamics can be favored a natural question is how robust the competing models are to a misspecification of these dynamics. Our simulation results suggest that the RSC relying on a regime switching framework, the SDCM assuming smoothly changing dependence parameter and SCAR model assuming autoregressive stochastic dependence seem to work better than those competing techniques that have been studied in more detail, also in situations when they are clearly misspecified. However, the DCC-copula model also performs quite well and given that its estimation is easy its use can be recommended in many situations. Table 4.7 gives an overview of the properties of the techniques under different criteria. Overall, if we had to recommend a single model it would be the RSC since in addition to good performance in the simulations it is easy to program and does not require heavy computations.

For assessing the goodness-of-fit we recommend comparing the log-likelihood statistics in addition 
Table 4.7: Comparison of the presented models

\begin{tabular}{rcccccc}
\hline \hline & DCC & PATT & SDCM & LCP & SCAR & RSC \\
estimating $\theta_{t}$ & $+/ 0$ & - & + & - & + & + \\
GoF testing & $+/ 0$ & - & $+/ 0$ & - & + & + \\
computations & + & + & - & - & - & + \\
flexibility & 0 & 0 & + & - & + & + \\
\hline \hline
\end{tabular}

to performing the Anderson-Darling test on the data transformed by the Rosenblatt probability integral transform, which has acceptable size and power properties for a number of models. However, ignoring the time-variation of the dependence when deciding which copula best fits the data is not recommendable as it will most likely lead to false conclusions.

In our empirical application we found that when allowing for time-varying dependence parameters symmetric copulas that do not allow for tail dependence offer the best fit, which is in contrast to what has been found in the literature for the static case, where usually copulas that feature tail dependence and asymmetry seem appropriate. Thus it appears that part of the asymmetry may be generated by time-varying parameters. The lack of tail dependence may partially be offset by the possibility of large overall dependence, which would explain why the Gaussian and Frank copulas fit the data so well. Finally, the models we studied seem to be reliable when estimating the Value-at-Risk.

The most important challenge for future research is to develop time-varying copula models in dimensions larger than two. This is crucial in order to make these models applicable for practical purposes. For Gaussian and Student copulas techniques from multivariate volatility modeling such as the DCC model and the model by Asai and McAleer (2009) look promising. Nevertheless, for non-elliptical dependence structures extensions are far from obvious and more research needs to be done. Further, methods to obtain multi-step forecasts of the dependence parameter have not been studied thoroughly in the literature, with the exception of the DCC-GARCH and the SCAR models, for which known results on autoregressive models can be used. Finally, goodness-of-fit techniques that help deciding which specification for the time-variation to chose need to be developed to avoid making to strong assumptions on the way dependence changes over time. 



\section{Testing for asset market linkages:}

\section{A new approach based on time-varying copulas ${ }^{1}$}

\subsection{Introduction}

The recent financial crises have renewed the debate on the importance of vulnerability of international financial markets and the propagation of foreign shocks. These turmoils originate from a crash in a "ground-zero" country ${ }^{2}$ which spreads across the world, even though market analysts considered other countries "healthy" before the crisis.

Financial market linkages are fostered by the trend towards an almost complete capital market liberalization and are consequently at the heart of the recent crises. Emerging countries wishing to finance domestic investments can find the capital they require on foreign capital markets. They are thus no longer bounded by their national saving and can accelerate their growth. Nevertheless, it is achieved at the cost of a higher risk of financial instability: a negative shock in a "ground-zero" country will be quickly and strongly transmitted to its financial partners. The group of crisiscontingent theories explain the increase in market cross-correlation after a shock issued in a "groundzero" country in several ways: multiple equilibria based on investor psychology, endogenous liquidity shocks causing a portfolio recomposition, and/or political disturbances affecting the exchange rate regime. The transmission of the crisis and the subsequent increase in cross correlation between markets is thus characterized by a "spill-over" or "shift-contagion" process. ${ }^{3}$ These crisis-contingent theories do not specify the channels of transmission which are assumed to be unstable and crisisdependent. In contrast, the non-crisis-contingent theories consider that the propagation of shocks

\footnotetext{
${ }^{1}$ This chapter is based on Candelon and Manner (2007).

${ }^{2}$ Thailand and the devaluation of the Thai Bath in July 1997 is considered as the event that initiated the Asian crisis.

${ }^{3}$ Masson (1999) considers the particular case of "false" shift-contagion, where the increase in cross-correlation may be due to the simultaneous occurrence of macroeconomic shocks across countries. According to the "monsoonal effect" theory, this artefact for shift-contagion is likely to happen as macroeconomic shocks are correlated.
} 
does not lead to a shift from a good to a bad equilibrium, but that the increase in cross-correlation is the continuation of linkages (trade and/or financial) existing before the crisis.

Empirical tests for "shift-contagion" avoid the identification of transmission channels and focus their attention on changing patterns of cross-market correlation. For example, the propagation of the Asian crisis from Thailand to Indonesia is revealed by a higher correlation between returns of these financial markets during the crisis period. This correlation breakdown has been considered by several empirical studies. For example, King and Wadhwani (1990) and Lee and Kwang (1993) show that financial market cross-correlation in the largest financial markets exhibits a significant increase after the U.S. stock market crash. Similarly Calvo and Reinhart (1995) and Baig and Goldfajn (1998) offer similar results after the 1994 Mexican and the 1997 Asian crises.

The concept of "shift-contagion" appeared to be a robust standard stylized fact until the influential paper of Forbes and Rigobon (2002). Considering a simple linear framework they show that any increase in correlation may be spurious and caused by a change in volatility. As during a crisis financial markets are subject to high volatility regimes, Forbes and Rigobon (2002) propose a stability test for correlation, which corrects for the influence of volatility changes. Applied to the 1994 Mexican and the 1997 Asian crises, the hypothesis of higher cross-market linkages is ruled out. Several recent studies have extended the framework proposed by Forbes and Rigobon (2002), see among others Candelon, Hecq, and Verschoor (2005), Corsetti, Pericoli, and Sbracia (2005) and Dungey and Zhumabekova (2001), ruling out the idea of absence of contagion.

The linear framework used in Forbes and Rigobon (2002) is also subject to strong criticism. Hartman, Straetmans, and de Vries (2004), Bae, Karolyi, and Stulz (2003) stress that contagion is not characterized by an increase in correlation over the whole sample, but only during a period of extreme events, i.e. a financial crises. It would support the idea that contagion is a transitory process and that dependence between markets deviates only temporarily from its long-run path. They consequently propose to test for an increase in tail dependence (also called "co-exceedence") around the financial crisis dates. ${ }^{4}$

Other studies such as Ramchand and Susmel (1998) and Ang and Bekaert (2002) to name but a few, prefer to consider another non-linear framework, namely the Markov-switching approach. They test for differences of the sample correlations among different volatility regimes identified as crisis and non-crisis periods. Maximum likelihood techniques are used to estimate the coefficients of a SWARCH and the probability matrix of staying or leaving a particular volatility regime.

\footnotetext{
${ }^{4}$ Formal tests for the stability tail-dependence are proposed in Straetmans (1997).
} 
More recently, Rodriguez (2007) investigates contagion using the concept of copulas. A copula is the part of a joint distribution that completely describes its dependence structure. Copulas allow the modeling of the dependence between variables in a flexible way and independently of the marginal distribution. Using copulas it is possible to model different dependencies for losses and gains (asymmetric dependence) and dependence of extreme events (tail dependence). They thus appear to allow for a more general characterization of contagion than linear correlation. Using asymmetric copulas and assuming that the same regimes govern the volatility and the (tail-)dependence structure, Rodriguez (2007) finds support for changing tail-dependence during periods of turmoil.

This chapter proposes to use copulas in order to investigate asset market shift-contagion. It is worth noticing that contagion is defined here as a significant increase in dependence, or correlation, as in Forbes and Rigobon (2002). This point of view differs from Rodriguez (2007), who focuses on tail-dependence. Moreover, we do not impose the change in dependence to coincide with the changes in volatility regime. It may be that both dates are identical, supporting the idea that change in dependence is synchronized with change in volatility regime. However, because of propagation time or information transfer, they are in our opinion unlikely to perfectly coincide. Typically, when tensions occur on the "ground zero" market we expect the linkage with the other markets to remain constant or even to slightly decrease until the transmission of the crisis is complete. Consequently, interdependence would rise to become higher than before the turmoil. The existence of such a "Jshape" can be addressed within the framework proposed in this chapter.

For this reason, this chapter contributes to the literature by setting up a sequential algorithm. Based on a time-varying copula, it allows for an efficient joint estimation of distinct breakpoints in dependence and volatility. To this end we elaborate on Dias and Embrechts (2004), who propose a formal test for the presence of a structural break in the dependence at an unknown period of time. The sequential algorithm also includes a formal estimation of the date of break in the variance parameters. Then, a Monte-Carlo study shows that the sequential algorithm exhibits correct size and power properties whatever the type of copula. In the empirical application, which deals with the Asian 1997 crisis, it turns out that contagion, defined as an increase in correlation, is a dominating feature among the Asian economies. Furthermore, the assumption that dependence and volatility exhibit a simultaneous change in regime is rejected. The date of the change in regime is different, supporting the idea that transmission process may take some time after the occurrence of a financial crisis.

The rest of the chapter is organized as follows. Methodological tools are introduced in Section 
5.2. Section 5.3 is devoted to an extensive Monte-Carlo analysis. Section 5.4 presents the results of our empirical study in the case of the Asian crisis and Section 5.5 offers some conclusions.

\subsection{Methodology}

\subsubsection{Copulas}

Copulas are multivariate distribution functions, which have uniform marginal distributions. They capture dependence between the random variables of interest independently of their marginal distributions and hence are scale invariant. Copulas find their applications mainly in finance when calculating the Value-at-Risk of a portfolio, pricing exotic options and credit derivatives, or for simply estimating the joint distribution of asset returns. ${ }^{5}$ In this study we only focus on bivariate copulas. Definitions and most results of bivariate copulas carry over to the multivariate setting. In practice, however, the extensions are trivial only for very specific cases and we thus limit the analysis to the bivariate case. The most important result on copulas, Sklar's theorem, can be found with a proof in Nelsen (2006) and states the following. Let $F$ be the marginal distribution function of $X$, $G$ be the marginal distribution function of $Y$, and let $H$ be the joint distribution function of $(X, Y)$. Then there exists a copula $C$ such that

$$
H(x, y)=C(F(x), G(y)), \forall(x, y) \in \overline{\mathbf{R}} \times \overline{\mathbf{R}}
$$

where $\overline{\mathbf{R}}$ denotes the extended real line. If $F$ and $G$ are continuous then $C$ is unique. Conversely if we have distribution functions $F$ and $G$ and a copula $C$, then $H$ is a bivariate distribution function. Recalling the probability integral transform for continuous distributions, which states that the random variable $U=F(X)$ has a $U(0,1)$ distribution regardless of the original distribution $F$, it becomes clear that a copula is no more than a multivariate distribution function with uniform marginals. It captures all the dependence between random variables of interest, as all the dynamics of the marginal distributions are captured by $F$ and $G$ for $X$ and $Y$, respectively. In the case of bivariate normal distribution, $F$ and $G$ are just normally distributed and the copula is completely described by the correlation between the margins. Other copulas allow for more complex and possibly non-linear dependence structures. For formal introductions to copulas and related functions, as well as a large

\footnotetext{
${ }^{5}$ For a good exposition of financial applications of copulas see Cherubini et al. (2004). Applications in settings apart from measuring financial risk are rather rare and examples can be found in Granger, Teräsvirta, and Patton (2006) for modeling the income consumption relationship or Bonhomme and Robin (2006) who model earnings trajectories with the help of copulas.
} 
number of examples of copulas we refer to the books by Joe (1997) and Nelsen (2006).

Patton (2006b) extended the theory by allowing the copula to be time varying and to depend on a conditioning set $\mathscr{F}_{t-1}$. In this way both the functional form of the copula and the copula parameter may vary over time. It is crucial, however, that the conditioning set is the same for marginal distributions as for the copula, since otherwise the extension of Sklar's theorem to conditional distributions is not valid.

\subsubsection{The model}

This chapter opts for a model of contagion and interdependence between two asset markets, which consists in keeping the conditional mean process a simple linear process and in considering timevarying copula based distributions for the error terms, evolving with volatility and correlation regimes. To this aim, the class of semiparametric copula-based multivariate dynamic (SCOMDY) models by Chen and Fan (2006a) is considered. They propose a parametric estimation the conditional mean and variance of multivariate time series (using VAR or AR and GARCH models). In contrast, the multivariate distribution of the innovations standardized by the conditional standard deviation is estimated via a semiparametric copula model. Surprisingly, the estimation of the first step model asymptotically does not influence the estimation of the copula parameter provided it is correctly specified. The model for the conditional mean is given by the following stationary VAR model:

$$
\mathbf{R}_{\mathbf{t}}=\boldsymbol{\Gamma}(\mathbf{L}) \cdot \mathbf{R}_{\mathbf{t}-\mathbf{1}}+\epsilon_{t}
$$

where $\mathbf{R}_{\mathbf{t}}$ are the stacked returns in markets $r_{1}$ and $r_{2}, \mathbf{R}_{\mathbf{t}}=\left[\begin{array}{ll}r_{1 t} & r_{2 t}\end{array}\right]^{\prime}$ and $\boldsymbol{\Gamma}(\mathbf{L})$ is a lag polynomial with roots lying outside the unit circle. $\epsilon_{t}=\left(\epsilon_{1 t}, \epsilon_{2 t}\right)^{\prime}$ are the VAR errors which have the following conditional distribution:

$$
\left(\begin{array}{c}
\epsilon_{1 t} \\
\epsilon_{2 t}
\end{array}\right) \mid \mathscr{F}_{t-1} \sim C\left(F\left(\epsilon_{1 t} ; \boldsymbol{\eta}_{\boldsymbol{t}}\right), G\left(\epsilon_{2 t} ; \boldsymbol{\eta}_{\boldsymbol{t}}\right) ; \boldsymbol{\theta}_{\boldsymbol{t}}\right)
$$

where $\mathscr{F}_{t-1}$ is the $\sigma$-field generated by the past returns. The variances of the marginal series $\sigma^{2}$ are included in the parameter vector $\boldsymbol{\eta}_{\boldsymbol{t}}=\left[\sigma_{t}^{2}, \boldsymbol{\xi}^{\prime}\right]^{\prime}$, where $\boldsymbol{\xi}$ captures the other moments of the marginal distribution, which are assumed constant over time and are treated as nuisance parameters. The copula is chosen to provide the best fit to the data, which is the one giving the smallest value for the Akaike information criterion (AIC). The dimension of the copula parameter $\boldsymbol{\theta}_{\boldsymbol{t}}$ depends on which 
copula is considered.

The marginal distributions $F$ and $G$ may be specified parametrically or non-parametrically. We allow the conditional volatility to follow a GARCH process, but we leave the distribution of the standardized innovations unspecified and model it by the empirical distribution function

$$
\hat{F}(x)=\frac{1}{T} \sum_{t=1}^{T} \mathbf{1}_{\left\{X_{t} \leqslant x\right\}} .
$$

Furthermore, we allow for a single breakpoint in the unconditional variance. Similarly, the copula parameter $\boldsymbol{\theta}_{\boldsymbol{t}}$ is characterized by a single (unknown) breakpoint. Alternatively, it can dynamically evolve over time as proposed in Patton (2006b), which will be described below. Both the breakpoints in volatility and in correlation are determined endogenously. Thus, changes in volatility and correlation induced by the regime shift are captured in the conditional distribution of the $V A R$ residuals.

Using copulas has three main advantages compared to using a known multivariate distribution such as the multivariate normal or student t-distribution. First, the individual series are likely to be not normally distributed (i.e. leptokurtic and skewed). The marginals underlying standard multivariate distributions do not allow for these features. Leaving the marginal distributions unspecified, eliminates the risk of misspecification, which may influence the estimation of the dependence parameter. Second, the dependence between two stock markets may show tail dependence (dependence of extreme losses), which may be modeled by several types of copulas. ${ }^{6}$ Third, using a copula representation as in (5.2) allows sequential estimation of marginal distribution as well as the copula itself, which is the basis of our test for a breakpoint in the dependence parameter conditional on a break in volatility. It also leads to a significant decrease in the computing time.

A test for contagion can then be performed as follows: First, one searches for a breakpoint in the dependence parameter. If correlation does not increase, there is clearly no evidence for shiftcontagion. When a breakpoint in the dependence parameter is detected, it may support the hypothesis of contagion, but it may also simply be due to an increase in volatility, see Forbes and Rigobon (2002). To discriminate between these possibilities, several methods are available. First, it is possible to compare the confidence intervals for the breakpoint in variance in the "ground-zero" country and those for the breakpoint in correlation. Second, the model (5.3) is extended to allow for the dependence parameter to vary over time, conditional on volatility. It then becomes possible to

\footnotetext{
${ }^{6}$ However, tail dependence analysis will not constitute the focus of this chapter.
} 
build a likelihood ratio test to determine whether the level or the regime of the conditional variance can explain the variation of the dependence parameter.

The tests for contagion based on correlation proposed by Forbes and Rigobon (2002) consist of estimating the $V A R$ model $(5.2)^{7}$ and restricting the distribution of the error terms to a bivariate normal distribution, which is estimated over two predetermined periods (one preceding and the other one during the turmoil). A significant increase in the correlation of the error term during the turmoil (i.e. $\left.\rho_{1}<\rho_{2}\right)$ indicates the presence of contagion. Several studies have elaborated on this seminal paper keeping a similar framework. ${ }^{8}$ This approach suffers from several drawbacks. First, it may be subject to sample selection bias as the choice of tranquil and crisis periods is done prior to the analysis. Second, the test assumes that the residuals are normally distributed. It is obvious that such a condition is violated in our case. Comparing the models before and during the crisis leads to the introduction of non-linearities, and transforms (5.2) into a regime-dependent model. Forbes and Rigobon (2002) associate these regimes to volatility stance, and propose a correction of the original test. It may nevertheless miss other explanatory factors and introduce again an endogeneity bias as the volatility regimes are determined beforehand.

\subsubsection{Testing for structural breaks in copula models (Dias and Embrechts 2004)}

A formal test for the presence of a breakpoint in the dependence parameter of a copula is developed by Dias and Embrechts (2004). They assume an independent sample $\left(x_{1}, y_{1}\right) \ldots\left(x_{T}, y_{T}\right)$ where $t=1, \ldots ., T$ generated by the bivariate distribution functions $H\left(x, y ; \boldsymbol{\theta}_{1}, \boldsymbol{\eta}_{1}\right) \ldots H\left(x, y ; \boldsymbol{\theta}_{T}, \boldsymbol{\eta}_{T}\right)$. The $\boldsymbol{\theta}_{t}$ 's are the parameters of the underlying copula, whereas the $\boldsymbol{\eta}_{t}$ 's are the parameters of the marginal distributions and are treated as nuisance parameters. Formally, the null hypothesis of no structural break in the copula parameter becomes

$$
H_{0}: \boldsymbol{\theta}_{1}=\boldsymbol{\theta}_{2}=\ldots=\boldsymbol{\theta}_{T} \text { and } \boldsymbol{\eta}_{1}=\boldsymbol{\eta}_{2}=\ldots=\boldsymbol{\eta}_{T}
$$

whereas the alternative hypothesis of the presence of a single structural break is formulated as:

$$
H_{1}: \boldsymbol{\theta}_{1}=\ldots=\boldsymbol{\theta}_{k} \neq \boldsymbol{\theta}_{k+1}=\ldots=\boldsymbol{\theta}_{T} \equiv \boldsymbol{\theta}_{k}^{*} \text { and } \boldsymbol{\eta}_{1}=\boldsymbol{\eta}_{2}=\ldots=\boldsymbol{\eta}_{T}
$$

\footnotetext{
${ }^{7}$ Forbes and Rigobon (2002) also add interest rate as an exogenous control variable.

${ }^{8}$ (5.2) can be restated without adding more information as a common factor representation in order to separate the common factor from the idiosyncratic country-specific component. Corsetti et al. (2005) extract the common factor using principal components whereas Candelon et al. (2005) perform a common feature approach. The test for contagion boils down to a stability analysis of the common component. If its weight is larger after the crisis it can be concluded that shift-contagion occurred.
} 
In the case of a known break-point $k$, the test statistics can be derived as a generalized likelihood ratio test. Let $L_{k}(\boldsymbol{\theta}, \boldsymbol{\eta}), L_{k}^{*}(\boldsymbol{\theta}, \boldsymbol{\eta})$ and $L_{T}(\boldsymbol{\theta}, \boldsymbol{\eta})$ be the log-likelihood functions of our copula given in (5.3) using the first $k$ observations, the observations from $k+1$ to $T$ and all observations, respectively. Then the likelihood ratio statistic can be written as

$$
L R_{k}=2\left[L_{k}\left(\hat{\boldsymbol{\theta}}_{k}, \hat{\boldsymbol{\eta}}_{T}\right)+L_{k}^{*}\left(\hat{\boldsymbol{\theta}}_{k}^{*}, \hat{\boldsymbol{\eta}}_{T}\right)-L_{T}\left(\hat{\boldsymbol{\theta}}_{T}, \hat{\boldsymbol{\eta}}_{T}\right)\right]
$$

where a hat denotes the maximizer of the corresponding log-likelihood function. Note that $\hat{\boldsymbol{\theta}_{k}}$ and $\hat{\boldsymbol{\theta}_{k}^{*}}$ denote the estimates of $\boldsymbol{\theta}$ before and after the break, whereas $\hat{\boldsymbol{\theta}}_{T}$ and $\hat{\boldsymbol{\eta}}_{T}$ are the estimates of $\boldsymbol{\theta}$ and $\boldsymbol{\eta}$ using the full sample. In the case of an unknown break date $k$, a recursive procedure similar to the one proposed by Andrews (1993) can be used. The test statistic is the supremum of the sequence of statistic for known $k$ :

$$
Z_{T}=\max _{1 \leqslant k<T} L R_{k}
$$

Dias and Embrechts (2004) recommend obtaining critical values using the approximation provided by Gombay and Horváth (1996), which we present here. Under $H_{0}$ it holds that for $T \rightarrow \infty$

$$
\left|Z_{T}^{1 / 2}-\sup _{1 / T \leqslant t \leqslant 1-1 / T}\left(\frac{B_{T}^{(d)}(t)}{t(1-t)}\right)^{1 / 2}\right|=o_{P}\left(\exp \left(-(\log (T))^{1-\varepsilon}\right)\right)
$$

for all $0<\varepsilon<1$, where $\left\{B_{T}^{(d)}: 0 \leqslant t \leqslant 1\right\}$ is a sequence of stochastic processes such that $\left\{B_{T}^{(d)}: 0 \leqslant\right.$ $t \leqslant 1\} \stackrel{d}{=}\left\{B^{(d)}: 0 \leqslant t \leqslant 1\right\}$ for each $T$ and $B^{(d)}(t)=\sum_{1 \leqslant i \leqslant d} B_{i}^{2}(t)$, where $\left\{B_{s}: 0 \leqslant t \leqslant 1\right\}, s=$ $1, \ldots, d$ are independent Brownian bridges and $d$ is the dimension of the copula parameter. There is no simple closed form expression for the distribution in (5.5). The following approximation can be used in practice. For $0<h<l<1$

$$
\begin{aligned}
P\left(\sup _{h \leqslant t \leqslant 1-l}\left\{\frac{B_{T}^{(d)}(t)}{t(1-t)}\right\}^{1 / 2} \geqslant x\right)=\frac{x^{d} \exp \left(-x^{2} / 2\right.}{2^{d / 2} \Gamma(d / 2)} \times \\
\quad\left(\log \frac{(1-h)(1-l)}{h l}-\frac{d}{x^{2}} \log \frac{(1-h)(1-l)}{h l}+\frac{4}{x^{2}}+\mathscr{O}\left(\frac{1}{x^{4}}\right)\right),
\end{aligned}
$$

as $x \rightarrow \infty$. Note that this limiting distribution is identical to the one proposed in Andrews (1993), which applies in a more general context. The only difference is that Gombay and Horváth (1996) let the trimming parameters $l$ and $h$ depend on the sample size through $l(t)=h(t)=\log (t)^{3 / 2} / t$, whereas Andrews (1993) considers a constant trimming value. We thus opt for the use of the critical 
values tabulated by Andrews (1993) and to trim the first and last 15\% of the observations.

\subsubsection{Testing for a structural break in unconditional volatility}

We test for and locate a breakpoint in volatility using a quasi-likelihood ratio test. To this end we model the return data with a normal distribution that has a structural break in variance at an unknown point in time $p$. This does not mean that we assume the return data to be normally distributed, but the maximum likelihood estimate of the variance parameter $\sigma^{2}$ converges in probability to its pseudo-true value, which is the unconditional variance of the (sub-)sample.

Let $L_{p}(\sigma), L_{p}^{*}(\sigma)$ and $L_{T}(\sigma)$ be the log-likelihood function of the Gaussian distribution using the first $p$ observations, the observations from $p+1$ to $T$ and the whole sample, respectively. Again $\hat{\sigma}_{p}$ and $\hat{\sigma}_{p}^{*}$ stand for the estimates of $\sigma$ before and after the candidate breakpoint and $\hat{\sigma}_{T}$ is the estimate of $\sigma$ using the whole sample. Similar to the approach for testing for a breakpoint in correlation denoting

$$
L R_{p}=2\left[L_{p}\left(\hat{\sigma}_{p}\right)+L_{p}^{*}\left(\hat{\sigma}_{p}^{*}\right)-L_{T}\left(\hat{\sigma}_{T}\right)\right]
$$

the test statistic of interest is

$$
Z_{T}=\max _{1 \leqslant p<T} L R_{p}
$$

In practice the series should be demeaned over both subsamples, in order to remove the possible problems induced by different means. The asymptotic theory driving the behavior of the statistic $Z_{T}$ for i.i.d. data is similar to the test above and the same critical values can be used.

Besides, it is quite likely that conditional volatility exhibits clusters and then follows a GARCH process. In such a case, previous asymptotic theory is not valid anymore: the time variation of conditional volatilities affects the estimates of the unconditional volatilities in finite (sub-)samples and therefore also the distribution of the test statistic under the null hypothesis. Thus, in such a case, critical values will be simulated using the estimated GARCH model.

Once the break in variance $\hat{p}$ is estimated, it is necessary to build its confidence interval at a certain level. To this aim, we develop a bootstrap procedure. We draw two bootstrap samples from our data set before and after $\hat{p}$. The test for a structural break in the variance is then performed on the complete bootstrap sample, leading to the detection of a variance breakpoint $\hat{p}^{b}$, which may be different from $\hat{p}$. This procedure is repeated a sufficiently large number of times $B$ and the empirical $95 \%$ confidence interval is calculated from the distribution of $\hat{p}^{b}$ where $b=1, \ldots, B$. 


\subsubsection{Testing for structural breaks in dependence conditional on a break in volatility: a sequential algorithm}

The problem of testing for a structural break in the dependence parameter becomes more complicated when there is a breakpoint in the variances of the individual series. We take this into account by allowing for a breakpoint in the volatility parameter $\sigma_{t}^{2}$ at an unknown point in time $p$ both under the null of no change in dependence and under the alternative. The other moments of the marginal distribution, captured by $\boldsymbol{\xi}$, are still treated as nuisance parameters and are not allowed to change over time. Consequently, we test for a break in dependence conditional on the fact that a break in volatility has occurred. Formally, the null hypothesis of our conditional test is

$$
\begin{aligned}
H_{0}: & \boldsymbol{\theta}_{1}=\boldsymbol{\theta}_{2}=\ldots=\boldsymbol{\theta}_{T} \text { conditional to } \\
& \boldsymbol{\xi}_{1}=\boldsymbol{\xi}_{2}=\ldots=\boldsymbol{\xi}_{T} \equiv \boldsymbol{\xi} \\
& \sigma_{1}=\ldots=\sigma_{p} \neq \sigma_{p}+1=\ldots=\sigma_{T} \equiv \sigma_{p}^{*},
\end{aligned}
$$

versus

$$
\begin{aligned}
H_{1}: & \boldsymbol{\theta}_{1}=\ldots=\boldsymbol{\theta}_{k} \neq \boldsymbol{\theta}_{k+1}=\ldots=\boldsymbol{\theta}_{T} \equiv \boldsymbol{\theta}_{k}^{*} \text { conditional to } \\
& \boldsymbol{\xi}_{1}=\boldsymbol{\xi}_{2}=\ldots=\boldsymbol{\xi}_{T} \equiv \boldsymbol{\xi} \\
& \sigma_{1}=\ldots=\sigma_{p} \neq \sigma_{p}+1=\ldots=\sigma_{T} \equiv \sigma_{p}^{*} .
\end{aligned}
$$

Under the alternative a single break is present in both the dependence (at time $k$ ) and the variance (at time $p$ ). A time-varying copula model allows for a simultaneous estimation of the break in the variance and in the dependence parameter. Intuitively, the $L R$ statistics is calculated for all possible points $k$ and $p$. We then retain the supremum and compare the LR value to the simulated critical value at a fixed nominal size. More precisely, let us assume without loss of generality that $p<k$ and let $L_{k \backslash p}(\boldsymbol{\theta},[\sigma, \boldsymbol{\xi}])$ be the likelihood function of the joint distribution in our model (5.3) using the observations between $p$ and $k$. In this situation the LR statistic becomes

$$
L R_{k, p}=2\left[L_{p}\left(\hat{\boldsymbol{\theta}}_{k},\left[\hat{\sigma}_{p}, \hat{\boldsymbol{\xi}}\right]\right)+L_{k \backslash p}\left(\hat{\boldsymbol{\theta}}_{k},\left[\hat{\sigma}_{p}^{*}, \hat{\boldsymbol{\xi}}\right]\right)+L_{k}^{*}\left(\hat{\boldsymbol{\theta}}_{k}^{*},\left[\hat{\sigma}_{p}^{*}, \hat{\boldsymbol{\xi}}\right]\right)-L_{T}\left(\hat{\boldsymbol{\theta}}_{T},\left[\hat{\sigma}_{T}, \hat{\boldsymbol{\xi}}\right]\right)\right]
$$


The test statistic then has the following form

$$
S_{T}=\max _{\substack{1 \leqslant k<T \\ 1 \leqslant p<T}} L R_{k, p}
$$

This supremum statistics becomes more complicated when the variances of the two series exhibit a breakpoint at distinct points $p_{1}$ and $p_{2}$. In any case, the asymptotic distribution of the LR statistic $S_{T}$ will also depend on the estimation of the breakpoint in the variance parameter $\sigma^{2}$. The approximations provided by Andrews (1993) and Gombay and Horváth (1996) should be modified to take the uncertainty in the estimation of the variance break into account.

Estimating all three breakpoints jointly is computationally very demanding and we opt for a sequential procedure to estimate the variance and dependence breaks. The copula decomposition of a joint distribution allows us to first estimate the marginal distributions, including the breakpoint in variance, followed by the estimation of the copula, which greatly reduces the computational burden. Therefore, we apply a conditional test in the second step, estimating a breakpoint in the copula parameter conditional on a break in the variance. The breaks in variance as well as the $95 \%$ confidence intervals $I_{p_{1}}$ and $I_{p_{2}}$ are estimated in a first step using the method introduced in Section 5.2.4. ${ }^{9}$ In a second step both series are transformed into uniform variables $(\tilde{u}, \tilde{v})$ such that $\tilde{u}=\hat{F}(x)$ and $\tilde{v}=\hat{F}(y) . \hat{F}(\cdot)$ is the empirical probability integral transform which has a different form before and after the estimated breaks $p_{1}$ and $p_{2}$ :

$$
\begin{aligned}
& \hat{F}_{p_{1}}(x)=\frac{1}{p_{1}} \sum_{t=1}^{p_{1}} \mathbf{1}_{\left\{X_{t} \leqslant x\right\}} \\
& \hat{F}_{p_{1}^{*}}(x)=\frac{1}{\left(T-p_{1}\right)} \sum_{t=p_{1}+1}^{T} \mathbf{1}_{\left\{X_{t} \leqslant x\right\}}
\end{aligned}
$$

and

$$
\begin{aligned}
& \hat{F}_{p_{2}}(y)=\frac{1}{p_{2}} \sum_{t=1}^{p_{2}} \mathbf{1}_{\left\{Y_{t} \leqslant y\right\}} \\
& \hat{F}_{p_{2}^{*}}(y)=\frac{1}{\left(T-p_{2}\right)} \sum_{t=p_{2}+1}^{T} \mathbf{1}_{\left\{Y_{t} \leqslant y\right\}} .
\end{aligned}
$$

Finally, the structural break test of the copula parameter proposed by Dias and Embrechts (2004) is

\footnotetext{
${ }^{9} p_{1}$ and $p_{2}$ refer to the breaks in the first and second series, respectively.
} 
applied to the transformed data $(\tilde{u}, \tilde{v})$. Thus, we compute a similar test statistic with the following form

$$
S_{T}=\max _{1 \leqslant k<T} L R_{k}
$$

Assuming that the variance breaks are consistently estimated the resulting estimate (of the break in the correlation coefficient) is also consistent. However, the nuisance caused by the estimation error in the first step must be taken into account when obtaining the critical values for the sup $L R$ statistic in the second step. Furthermore, the stability test may exhibit a size bias when the breaks are close to the trimming value, see Candelon and Lütkepohl (2001). To tackle these potential problems the following parametric bootstrap algorithm is set up:

1. Sequentially estimate first the variance breaks $p_{1}$ and $p_{2}$, and then the correlation break conditional on the estimated variance breaks $k \mid \hat{p}_{1}, \hat{p}_{2}$. Store the $95 \%$ confidence intervals $I_{\hat{p}_{1}}$ and $I_{\hat{p}_{2}}$ for the variance breaks.

2. Estimate the time constant copula parameter $\bar{\theta}$

3. Randomly draw two points $p_{1}^{\prime}, p_{2}^{\prime}$ for the variance breaks from the confidence intervals $I_{\hat{p}_{1}}$ and $I_{\hat{p}_{2}}$.

4. Estimate GARCH parameters $\boldsymbol{\omega}_{p_{i}^{\prime}}$ and $\boldsymbol{\omega}_{p_{i}^{\prime}}$ before and after the drawn breaks for both series.

5. Generate two random series $(u, v)$ from a time constant copula $C(u, v ; \bar{\theta})$ and transform the marginal series into heteroscedastic variables using the estimated GARCH parameters before and after that break.

6. Apply the sequential breakpoint test to this series and compute the sup LR $Z^{*}$ statistic for the correlation break.

7. Repeat steps 3-6 $m$ times ( $m$ being sufficiently large) and obtain the desired empirical quantile from the bootstrapped test statistics.

Until now, for the sake of generality no specific type of copula is assumed. Nevertheless, in empirical work we suggest using the simple and well known Gaussian copula. Its distribution function is given by

$$
C^{\text {Gaussian }}(u, v)=\int_{-\infty}^{\phi^{-1}(u)} \int_{-\infty}^{\phi^{-1}(v)} \frac{1}{2 \pi \sqrt{1-\rho^{2}}} \exp \left(-\frac{s^{2}-2 \rho s t+t^{2}}{2\left(1-\rho^{2}\right)}\right) d s d t,
$$

where $\rho$ is the linear correlation coefficient of the corresponding bivariate normal distribution. The simulation results in the next section will show that breakpoints in the dependence parameter can 
be found consistently using the Gaussian copula even when the data are issued from data generating processes producing tail dependence or volatility clusters. The procedure is then quite robust to misspecification. Restricting the attention to the Gaussian copula is also justified by the fact that the asymmetric dependence often encountered in financial data and modeled by more flexible copulas seems to be taken into account partly by time-varying dependence.

\subsection{Monte Carlo study}

The purpose of this section is to study the performance of our testing procedure in small samples. To this aim, we retain three of the most frequent data generating processes (DGP's) encountered in empirical works.

The first $D G P(D G P(1))$ corresponds to a simple Gaussian copula with i.i.d. Gaussian marginals, i.e. a bivariate normal distribution. Thus the series $Y_{1, t}, Y_{2, t}$ have the distribution

$$
F\left(Y_{1, t}, Y_{2, t}\right)=C^{\text {Gaussian }}\left(\Phi\left(Y_{1, t}\right), \Phi\left(Y_{2, t}\right), \rho_{t}\right),
$$

where $\Phi(\cdot)$ denotes the CDF of the standard normal distribution. This distribution is the same as the one used in the sequential approach. Therefore, it is expected that under this DGP the LR approach for detecting a break in variance and dependence performs the best in terms of size and power.

The Monte Carlo experiments should also analyze the behavior of this testing procedure in front of DGP's relaxing the normality assumption. To this aim, $D G P(2)$ assumes volatility clusters in stock market returns series generated by univariate GARCH processes, whereas $D G P(3)$ allows for asymmetric dependence structures and tail dependence. To be more precise, in $D G P(2)$ let $\left(\Phi\left(\epsilon_{1 t}\right), \Phi\left(\epsilon_{1 t}\right)\right) \sim C^{\text {Gaussian }}$. Then $Y_{i, t}$ follows a GARCH process:

$$
Y_{i, t}=\sqrt{h_{i, t}} \epsilon_{i, t}
$$

where

$$
h_{i, t}=\omega_{i, t}+\alpha Y_{i, t-1}^{2}+\beta h_{i, t-1} .
$$

Such a process is frequent for financial series, which exhibits volatility clustering.

$D G P(3)$ corresponds to Clayton Copula with i.i.d. normal marginals and has the following form. 
The Clayton copula is given by

$$
C_{\theta}^{\text {Clayton }}(u, v)=\max \left[\left(u^{-\theta}+v^{-\theta}-1\right)^{\frac{-1}{\theta}}, 0\right],
$$

with $\theta>0$. To ensure comparability of the degree of dependence with the DGP's using the Gaussian copula, the parameter for the Clayton copula is chosen such that the two copulas have the same value for the concordance measure Kendall's $\tau$.

In a first experiment, the DGP's are simulated under the null hypothesis of no break in the dependence parameter (i.e. size analysis). Nevertheless, it is assumed that a single break in the variance occurs at date $p$, where $p=(1 / 4,1 / 2,3 / 4) \cdot T$ and $T$ is the sample size. The unconditional standard deviations before and after the breakpoint are set to $\sigma_{l}=1$ and $\sigma_{h}=3$, corresponding more or less to the estimates found in our empirical part. The sample sizes investigated are 500, 1000 and 2000.

Table 5.1 reports the rejection frequency of the test for a structural break in the dependence parameter in three cases. First, when the break in variance is ignored and applying the original Dias and Embrechts (2004) test (DE hereafter). Second, when this break is taken into account and following the sequential testing approach (STA hereafter) developed in Section 5.2.5 ${ }^{10}$. Third, when the volatility regimes are known and applying the famous Forbes and Rigobon (2002) test (FR hereafter). In all cases, the nominal size is fixed at $5 \%$.

It turns out that ignoring the break in variance leads to severe size distortion whatever the DGP considered. The rejection frequencies are always above the nominal size, indicating that the presence of a break in the dependence parameter is too often supported by DE in presence of volatility break. The importance of the size distortion increases with the sample size, but is not affected by the location of the variance break, indicating inconsistency of the test. ${ }^{11}$ This result corroborates the initial findings of Forbes and Rigobon (2002), who show that tests for dependence stability are heavily biased in the presence of volatility regimes. Nevertheless, FR does not appear as an adequate alternative when the DGP is copula based. Columns 8 to 10 of Table 5.1 show that the rejection frequency of the FR test is well below the nominal size. The null hypothesis of stability in the dependence is then too often supported, leading to reject the presence of shift contagion in almost all cases.

Columns 5 to 7 of Table 5.1 report the rejection frequency using STA approach, which takes into

\footnotetext{
${ }^{10}$ Critical values were obtained using the parametric bootstrap algorithm.

${ }^{11}$ It is nevertheless noticeable that the size bias is the lowest in the case of a variance break located at $p=3 / 4 \cdot T$.
} 
Table 5.1: Size of the stability test using DE and STA.

\begin{tabular}{l|rrr|rrr|rrrr}
\hline \hline & \multicolumn{4}{|c}{$\begin{array}{c}\text { DE } \\
\text { break in variance }(\mathrm{p})\end{array}$} & \multicolumn{4}{c}{$\begin{array}{c}\text { STA } \\
\text { ignored }\end{array}$} & \multicolumn{4}{c}{$\begin{array}{c}\text { FR } \\
\text { known }\end{array}$} \\
\hline break date* T & $1 / 4$ & $1 / 2$ & $3 / 4$ & $1 / 4$ & $1 / 2$ & $3 / 4$ & $1 / 4$ & $1 / 2$ & $3 / 4$ \\
\hline $\mathrm{DGP1}$ & & & & & & & & & \\
$\mathrm{T}=500$ & 0.601 & 0.605 & 0.543 & 0.051 & 0.059 & 0.06 & 0 & 0 & 0 \\
$\mathrm{~T}=1000$ & 0.731 & 0.758 & 0.667 & 0.061 & 0.052 & 0.064 & 0 & 0 & 0 \\
$\mathrm{~T}=2000$ & 0.850 & 0.860 & 0.788 & 0.053 & 0.048 & 0.055 & 0 & 0 & 0 \\
\hline $\mathrm{DGP2}$ & & & & & & & & & \\
$\mathrm{T}=500$ & 0.528 & 0.484 & 0.335 & 0.049 & 0.049 & 0.053 & 0.001 & 0 & 0 \\
$\mathrm{~T}=1000$ & 0.598 & 0.565 & 0.466 & 0.053 & 0.035 & 0.036 & 0 & 0 & 0 \\
$\mathrm{~T}=2000$ & 0.670 & 0.651 & 0.547 & 0.049 & 0.062 & 0.054 & 0 & 0 & 0 \\
\hline $\mathrm{DGP3}$ & & & & & & & & & \\
$\mathrm{T}=500$ & 0.611 & 0.656 & 0.564 & 0.073 & 0.061 & 0.083 & 0.002 & 0 & 0.002 \\
$\mathrm{~T}=1000$ & 0.752 & 0.767 & 0.699 & 0.079 & 0.06 & 0.075 & 0 & 0 & 0 \\
$\mathrm{~T}=2000$ & 0.832 & 0.857 & 0.798 & 0.083 & 0.074 & 0.068 & 0 & 0 & 0 \\
\hline \hline
\end{tabular}

Note: Size of the copula breakpoint test by Dias and Embrechts (2004) ignoring changes in volatility (columns 2 to 4 ) and the size of our copula breakpoint test procedure (columns 5 to 7 ). Nominal size is $5 \%$ and the number of replications is equal to 1,000 .

account the variance break. It turns out that the size is close to the nominal size of the test when considering $D G P(1)$. Such a result is not surprising for $D G P(1)$, as the Gaussianity hypothesis is at the basis of the sequential procedure. More interestingly, the size is also quite correct, even if slightly higher than $5 \%$ in the case of the Clayton copula $(D G P(3))$. It shows that STA is not too much affected by the presence of lower tail dependence. Intuitively, the dependence parameter of the Gaussian copula measures the overall degree of dependence. It is thus likely that stability tests are not affected by symmetric heavy tails and only marginally by asymmetric lower or higher tails. To conclude, the type of copula does not constitute a limiting factor for the STA approach, and thus the Gaussian copula will be used in the rest of the chapter. The size of STA in the case of $D G P(2)$ is also close to $5 \%$. Such an outcome is also expected as the critical values used in the test for structural break in unconditional volatility are obtained by simulations allowing for GARCH dynamics. ${ }^{12}$ All these conclusions hold whatever the sample size and the location of the variance break.

In a second experiment, the three DGP's and designs are kept identical except that the dependence parameter is subject to a structural break, occurring at date $k$, where $k=(1 / 4,1 / 2,3.4) \cdot T$. Before the break, $\rho_{l}=0.3$, and then increases to $\rho_{h}=(0.5,0.6,0.7)$. We are thus now investigating the

\footnotetext{
${ }^{12}$ The test for structural break in unconditional volatility can also be modified accordingly if one suspects asymmetric or fat tailed GARCH process. Critical values can be obtained by modifying the simulation technique and are available upon request for the case of a GARCH with Student-t innovations.
} 
power of the ST approach. Table 5.2 reports the rejection frequency of the test for structural break in the dependence parameter using the STA.

The power ${ }^{13}$ of the STA turns out to be quite high and of the same magnitude for the three DGP's under scope. Besides, as already noticed, it does not seem to depend on the location of the variance break. Finally, as expected, the power increases with the sample size $T$ and is maximum for breakpoints in the dependence parameter located at the middle of the sample.

Finally, from the previous experiment it is possible to determine the $95 \%$ confidence bound for $\hat{k}$, the break date in the dependence parameter. They are reported in Table 5.3. The STA appears to perform quite well in detecting the date of break in the dependence parameter. Not surprisingly, the width of the confidence bounds around the true value of the break date decreases with the sample size $T$.

To conclude, the STA based on a Gaussian copula appears as an adequate approach to test for a structural break in the dependence parameter, while accounting for the possible structural break in volatility.

\footnotetext{
${ }^{13}$ It is noticeable that power is not size corrected. Nevertheless, as the size distortions are really small (see Table 5.1) using ST, such a conclusion will remain valid when considering size adjusted power.
} 
Table 5.2: Power of the stability test using STA.

\begin{tabular}{|c|c|c|c|c|c|c|c|c|c|}
\hline \multirow[b]{2}{*}{ Break $\sigma^{*} \mathrm{~T}$} & \multicolumn{3}{|c|}{ Break $\rho: 1 / 4^{*} \mathrm{~T}$} & \multicolumn{3}{|c|}{ Break $\rho: 1 / 2^{*} \mathrm{~T}$} & \multicolumn{3}{|c|}{ "Break $\rho: 3 / 4^{* T}$} \\
\hline & $1 / 4$ & $1 / 2$ & $3 / 4$ & $1 / 4$ & $1 / 2$ & $3 / 4$ & $1 / 4$ & $1 / 2$ & $3 / 4$ \\
\hline \multicolumn{10}{|l|}{ DGP1 } \\
\hline \multicolumn{10}{|l|}{$\mathrm{T}=500$} \\
\hline$\rho_{h}=0.5$ & 0.509 & 0.528 & 0.532 & 0.642 & 0.646 & 0.654 & 0.496 & 0.464 & 0.525 \\
\hline$\rho_{h}=0.6$ & 0.917 & 0.938 & 0.928 & 0.973 & 0.977 & 0.975 & 0.917 & 0.917 & 0.924 \\
\hline$\rho_{h}=0.7$ & 0.999 & 1 & 0.999 & 1 & 1 & 1 & 1 & 0.999 & 0.999 \\
\hline \multicolumn{10}{|l|}{$\mathrm{T}=1000$} \\
\hline$\rho_{h}=0.5$ & 0.838 & 0.831 & 0.829 & 0.922 & 0.92 & 0.94 & 0.843 & 0.83 & 0.834 \\
\hline$\rho_{h}=0.6$ & 0.999 & 0.999 & 1 & 1 & 1 & 1 & 0.999 & 0.997 & 0.997 \\
\hline$\rho_{h}=0.7$ & 1 & 1 & 1 & 1 & 1 & 1 & 1 & 1 & 1 \\
\hline \multicolumn{10}{|l|}{$\mathrm{T}=2000$} \\
\hline$\rho_{h}=0.5$ & 0.995 & 0.992 & 0.994 & 1 & 1 & 1 & 0.99 & 0.99 & 0.989 \\
\hline$\rho_{h}=0.6$ & 1 & 1 & 1 & 1 & 1 & 1 & 1 & 1 & 1 \\
\hline$\rho_{h}=0.7$ & 1 & 1 & 1 & 1 & 1 & 1 & 1 & 1 & 1 \\
\hline \multicolumn{10}{|l|}{$\begin{array}{c}\text { DGP2 }\end{array}$} \\
\hline$\rho_{h}=0.5$ & 0.418 & 0.443 & 0.458 & 0.566 & 0.611 & 0.613 & 0.427 & 0.432 & 0.484 \\
\hline$\rho_{h}=0.6$ & 0.879 & 0.883 & 0.874 & 0.946 & 0.963 & 0.96 & 0.846 & 0.87 & 0.889 \\
\hline$\rho_{h}=0.7$ & 0.997 & 0.993 & 0.993 & 1 & 1 & 0.999 & 0.997 & 0.996 & 0.997 \\
\hline \multicolumn{10}{|l|}{$\mathrm{T}=1000$} \\
\hline$\rho_{h}=0.5$ & 0.783 & 0.776 & 0.751 & 0.88 & 0.896 & 0.88 & 0.755 & 0.786 & 0.817 \\
\hline$\rho_{h}=0.6$ & 0.997 & 0.992 & 0.994 & 0.999 & 1 & 1 & 0.989 & 0.998 & 0.997 \\
\hline$\rho_{h}=0.7$ & 1 & 1 & 1 & 1 & 1 & 1 & 1 & 1 & 1 \\
\hline \multicolumn{10}{|l|}{$\mathrm{T}=2000$} \\
\hline$\rho_{h}=0.5$ & 0.977 & 0.972 & 1 & 0.994 & 0.999 & 0.997 & 0.964 & 0.976 & 0.98 \\
\hline$\rho_{h}=0.6$ & 1 & 1 & 0.999 & 1 & 1 & 1 & 1 & 1 & 1 \\
\hline$\rho_{h}=0.7$ & 1 & 1 & 1 & 1 & 1 & 1 & 1 & 1 & 1 \\
\hline \multicolumn{10}{|l|}{$\overline{\text { DGP3 }}$} \\
\hline \multicolumn{10}{|l|}{$\mathrm{T}=500$} \\
\hline$\rho_{h}=0.5$ & 0.495 & 0.523 & 0.539 & 0.622 & 0.673 & 0.642 & 0.448 & 0.487 & 0.505 \\
\hline$\rho_{h}=0.6$ & 0.888 & 0.901 & 0.894 & 0.943 & 0.948 & 0.95 & 0.873 & 0.867 & 0.901 \\
\hline$\rho_{h}=0.7$ & 0.995 & 0.997 & 0.997 & 0.999 & 1 & 1 & 0.994 & 0.993 & 0.994 \\
\hline \multicolumn{10}{|l|}{$\mathrm{T}=1000$} \\
\hline$\rho_{h}=0.5$ & 0.804 & 0.818 & 0.822 & 0.91 & 0.911 & 0.925 & 0.773 & 0.811 & 0.821 \\
\hline$\rho_{h}=0.6$ & 0.992 & 0.995 & 0.997 & 1 & 1 & 0.999 & 0.998 & 0.99 & 0.995 \\
\hline$\rho_{h}=0.7$ & 1 & 1 & 1 & 1 & 1 & 1 & 1 & 1 & 1 \\
\hline \multicolumn{10}{|l|}{$\mathrm{T}=2000$} \\
\hline$\rho_{h}=0.5$ & 0.985 & 0.977 & 0.987 & 0.997 & 1 & 0.997 & 0.977 & 0.979 & 0.988 \\
\hline$\rho_{h}=0.6$ & 1 & 1 & 1 & 1 & 1 & 1 & 1 & 1 & 1 \\
\hline$\rho_{h}=0.7$ & 1 & 1 & 1 & 1 & 1 & 1 & 1 & 1 & 1 \\
\hline
\end{tabular}

Note: Power of STA for all three DGP's. Nominal size is $5 \%$ and the number of replications is equal to 1,000 . 


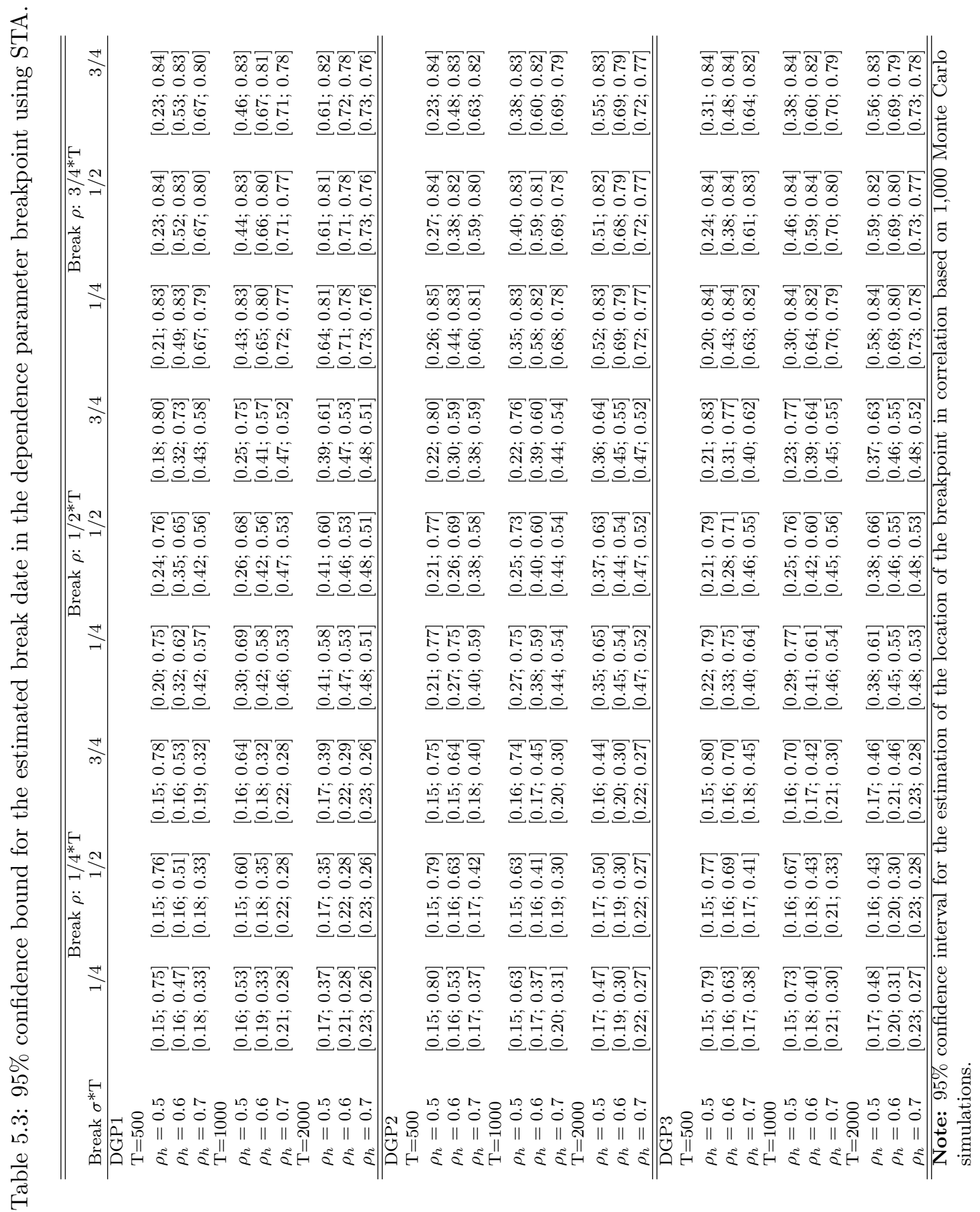




\subsection{Empirical application}

The financial turmoil that has affected Asian countries in 1997 has fueled the empirical literature on contagion, see Dungey, Fry, and Martin (2006) or Candelon, Piplack, and Straetmans (2008). Its main feature is that Asian countries were assumed to be well behaved, i.e. to possess strong macroeconomic fundamentals, before the occurrence of the crisis, leaving market analysts or modelers of the first generation without any voice. The importance of the transmission of the crisis from Thailand, which was first hit, to the rest of Asia is therefore at the core of this global crisis. The conclusion of Forbes and Rigobon (2002) rejects the presence of contagion in this crisis, and has left analysts skeptical. ${ }^{14}$ However, their test tends to be biased if the break in variance and correlation are erroneously assumed to be identical. In such a case the sequential procedure based on time-varying copula presented in Section 5.2 may be the best alternative.

\subsubsection{Data}

In this empirical application, stock returns for eight Asian countries ${ }^{15}$ are considered. Series are daily, extracted from Datastream market indices, labeled in $U S \$$ and cover the period 01/01/1996 30/06/1998, i.e. 652 observations. Returns of the market indices are plotted in Figure 5.1.

To shed new light on the question of the possible contagious characteristic of the Asian crisis, the ST approach, presented in Section 5.2.5, is applied. We consider both Thailand and Hong Kong as the "ground-zero" countries at the origin of the financial crisis. It is well-documented in the literature, see for example Dungey et al. (2006), that the Asian crisis was first initiated by the Thai Baht devaluation, and then by the crash of the Hong Kong stock market. These shocks have to be analyzed separately to draw a global picture of the shift-contagious process during the Asian crisis.

\subsubsection{The Sequential Testing Approach (STA)}

For each bivariate system of the other 6 Asian countries with both Thailand and Hong Kong (i.e. 13 models), we estimate the VAR given in model 5.2 and then the steps $1-7$ are performed on the residuals. ${ }^{16}$ Note that following the earlier discussion and the outcomes of the Monte-Carlo

\footnotetext{
${ }^{14}$ Some other empirical papers extending the Forbes and Rigobon (2002) test reach a different conclusion. See Corsetti et al. (2005) and Candelon et al. (2005).

${ }^{15}$ These countries are Thailand, Malaysia, Japan, Hong-Kong, Taiwan, Indonesia, Korea and the Philippines.

${ }^{16}$ The study is limited to bivariate systems in order to avoid the possible effect of indirect contagion or third-country effects. The Johansen cointegration tests reject the presence of cointegration relationships for the bivariate systems composed by the indices, allowing us to specify the VAR for the return series. Furthermore, optimal lag length is determined according to the Schwarz information criterion.
} 
Figure 5.1: Returns series
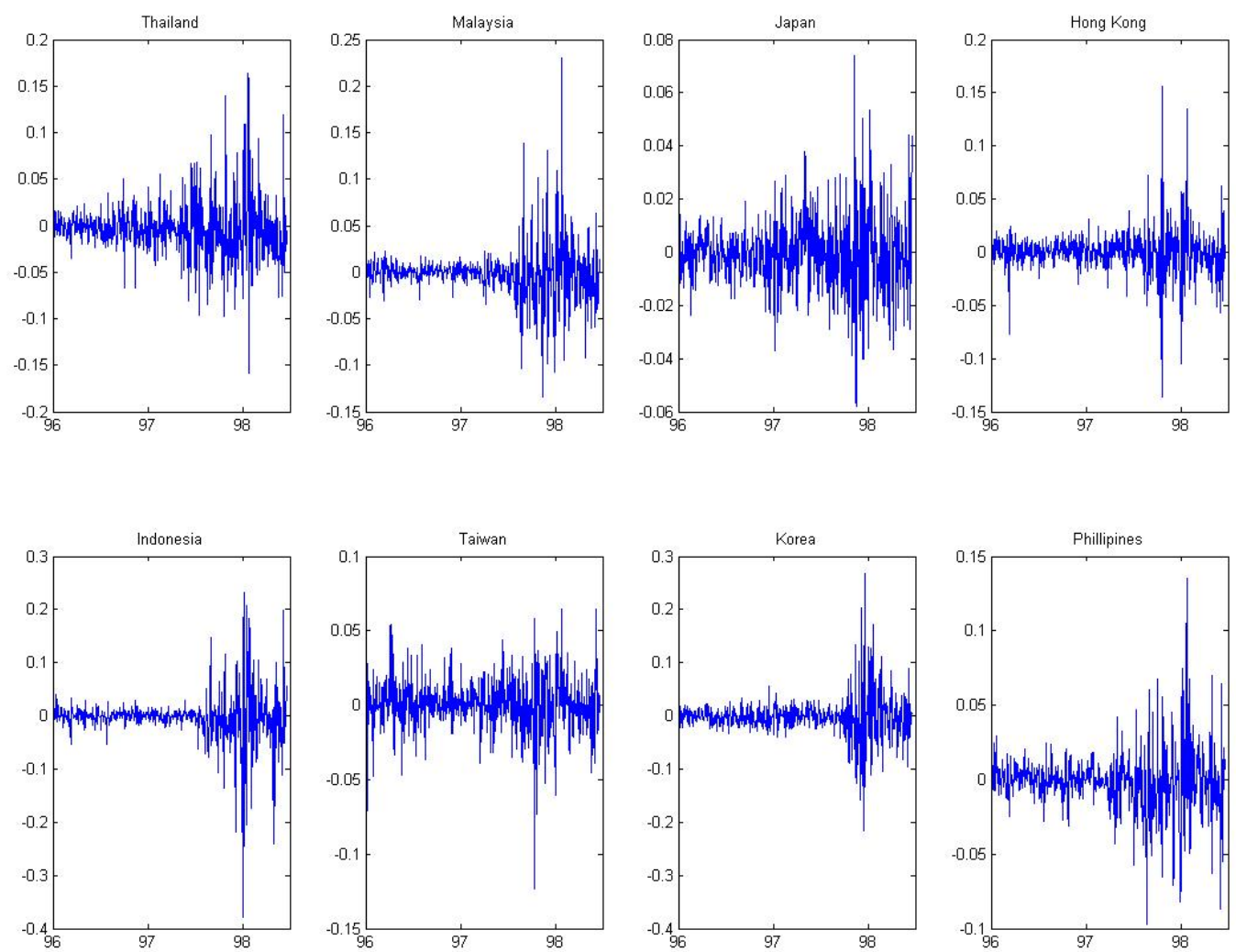

experiments, only Gaussian copulas are considered for sake of simplicity. Table 5.4 reports the dates for the structural breaks found in all univariate systems, and Table 5.5 gathers the dates for the structural breaks in the conditional copula, together with confidence intervals, which together should allow for a conclusion whether contagion has occurred.

It first turns out that point estimates for the dates of the break in variance generally precede the ones from of the conditional correlations. ${ }^{17}$ There are two cases, one where the confidence intervals of the variance and correlation breaks overlap and one where they do not. The assumption of concomitance between the change in volatility and dependence is thus not always supported. It supports the intuition that some time elapses between the occurrence of the crisis, the increase in volatility and its spill-over developments. This delay finds its justification in the time for information to be spread over the region. Typically, tensions occurring on the "ground zero" reduce the dependence with the

\footnotetext{
${ }^{17}$ Asides from comparison of break dates in the variance and the conditional correlation, the "J-shape" form of the conditional correlations, which is plotted in Figure 5.3 constitutes another support for this conclusion.
} 
Table 5.4: Breakpoints in variance.

\begin{tabular}{lc}
\hline \hline Thailand & $12-5-1997$ \\
Malaysia & {$[24-4-1997,2-6-1997]$} \\
& $30-7-1997$ \\
Japan & {$[28-7-1997,6-8-1997]$} \\
& $3-12-1996$ \\
Japan second break & {$[22-11-1996,26-12-1996]$} \\
& $20-10-1997$ \\
Hong Kong & $11-9-1997,5-2-1998]$ \\
& $15-8-1997$ \\
Indonesia & $114-7-1997,5-9-1997]$ \\
& $12-8-1997$ \\
Taiwan & {$[5-8-1997,18-8-1997]$} \\
& $18-7-1997$ \\
Korea & {$[24-1-1997,8-12-1997]$} \\
& $13-10-1997$ \\
Philippines & {$[9-10-1997,22-10-1997]$} \\
& {$[11-4-1997,1-59-1997]$} \\
\hline \hline
\end{tabular}

Note: This table reports the date of the structural break in volatility found using the sequential approach, describe in Section 5.2.4. Confidence bounds are bootstrapped (1,000 replications) and indicated between brackets.

other markets until the transmission of the crisis. Then, linkages would rise to become higher than before the turmoil. Moreover, such a result justifies our sequential testing procedure, which tends to support contagion for all the systems except (Hong Kong; Japan) and (Hong Kong; Korea), for which no significant change in conditional correlation, is detected. It is nevertheless clear that these two systems are a minority compared to the 11 other exhibiting a significant break. Thus, the main picture delivered by our study is clearly in favor of the existence of contagious characteristics of the Asian 1997 crisis. 


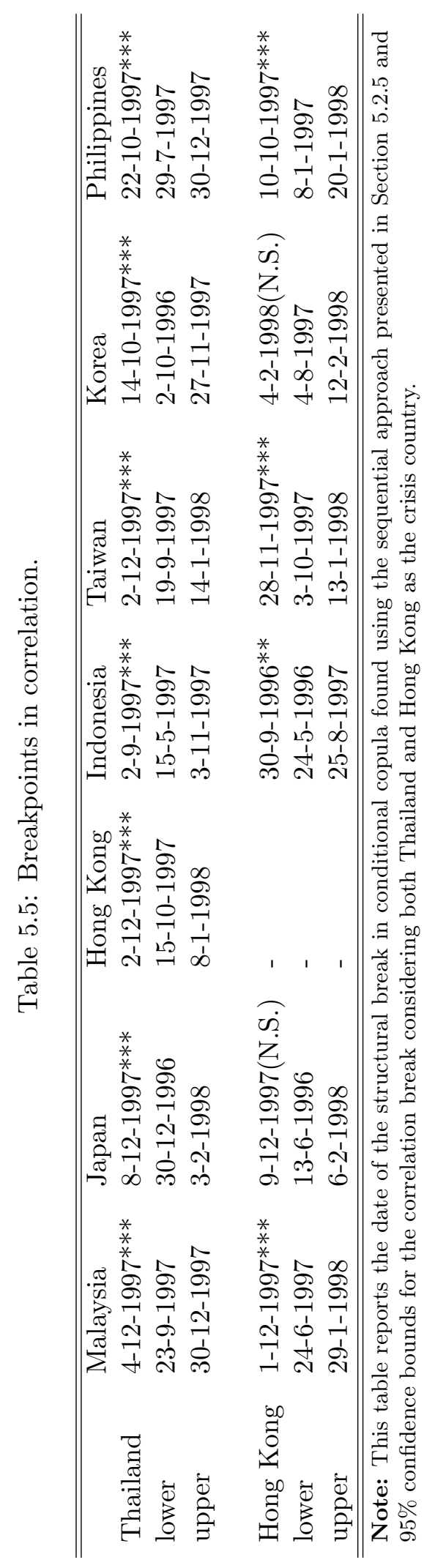




\subsubsection{Modeling time-varying dependence}

Next, a log-likelihood ratio test is used to evaluate the relative significance of the volatility and the correlation regime (characterized by two different dummies) in the time-varying copula parameter. To this aim the conditional mean is modeled via a VAR model given in (5.2). The conditional variance $h_{t}$ of the errors $\epsilon_{t}$ is modeled by a t-GARCH, which can be stated as

$$
h_{t}=\omega_{t}+\alpha_{1} \epsilon_{t-1}^{2}+\alpha_{2} h_{t-1}
$$

where the standardized innovations $\nu_{t}=\sqrt{\frac{\kappa}{h_{t}(\kappa-2)}} \nu_{t} \sim t_{\kappa}$. The $\nu_{t}$ 's are modeled by a semiparametric Gaussian copula model, where the correlation parameter is allowed to vary over time conditional on a set of variables. Formally,

$$
\left(\begin{array}{c}
v_{1 t} \\
v_{2 t}
\end{array}\right) \mid \Omega_{t-1} \leadsto C^{\text {Gaussian }}\left(\hat{F}\left(v_{1}\right), \hat{F}\left(v_{2}\right) ; \rho_{t}\right),
$$

where $\hat{F}$ denotes the empirical probability integral transform. Recall that $p$ is the breakpoint in variance of the "ground-zero" country and $k$ is the break of the copula parameter estimated previously. Define two dummies $D_{t}(\rho)$ and $D_{t}(\sigma)$ as: $D_{t}(\sigma)=0$ for $t<p, D_{t}(\sigma)=1$ for $t \geqslant p, D_{t}(\rho)=0$ for $t<k$ and $D_{t}(\rho)=1$ for $t \geqslant k$. Then $\rho_{t}$ evolves over time according to

$$
\rho_{t}=\Lambda\left(\alpha+\beta_{1} \cdot D_{t}(\sigma)+\beta_{2} \cdot D_{t}(\rho)\right)
$$

where $\Lambda(x)=\frac{1-e^{-x}}{1+e^{-x}}$ is the modified logistic transformation to keep $\rho$ in $(-1,1) .{ }^{18}$ We test the two restrictions that one of the two dummies is not significant, given the other is included in the model. Formally, the sequence of hypotheses is the following one:

$H_{0}^{a}: \beta_{2}=0$

and

$H_{0}^{b}: \beta_{1}=0$

against:

$H_{1}: \beta_{1} \neq 0$ and $\beta_{2} \neq 0$

\footnotetext{
${ }^{18}$ The conditional variance estimated from a GARCH model can also be used as the measure for volatility instead of the dummy. The results were very similar to the ones presented here and are available upon request.
} 
Table 5.6: Likelihood ratio tests for significance of variance and correlation break dummies.

\begin{tabular}{crrrrrrrr}
\hline \hline \multirow{2}{*}{ Thailand } & & Malaysia & Japan & Hong Kong & Indonesia & Taiwan & Korea & Philippines \\
& $H_{0}^{a}$ & 0 & 0 & 0 & 0.0011 & 0.0003 & 0.004 & 0 \\
& $H_{0}^{b}$ & 0.0658 & 0.2827 & 0.0002 & 0.5368 & 0.2249 & 0.5668 & 0.0309 \\
\hline Hong Kong & $H_{0}^{a}$ & 0.0169 & 0.3581 & - & 0 & 0.0027 & 0.0907 & 0.0077 \\
& $H_{0}^{b}$ & 0.5381 & 0.7328 & - & 0.0026 & 0.3171 & 0.0696 & 0.4516 \\
\hline \hline
\end{tabular}

Note: This table reports the p-value for a LR test of a dummy capturing the correlation (variance) breakpoint, given a dummy for the variance (correlation) break is also included in the model.

The p-values for the tests are given in Table 5.6. In almost all the cases $H_{0}^{a}$ is rejected against $H_{1}$, indicating that the correlation dummy improves the model when the variance dummy is included. However, $H_{0}^{b}$ is rejected in favor of $H_{1}$ only in a few cases. The presence of a dummy for the variance hence is not found to be significant when a correlation break dummy is already in the model. Taken together these results support the previous finding of shift-contagion, irrespective of the presence of a change in volatility.

As a final step in our analysis we use conditional copulas to model the path of the correlation coefficient of a Gaussian copula for the standardized VAR-tGARCH residuals, transformed by the empirical distribution function. To this end the correlation coefficient evolves as proposed in Patton (2006b):

$$
\rho_{t}=\Lambda\left(\alpha+\beta_{1} \cdot \rho_{t-1}+\beta_{2} \cdot \frac{1}{p} \sum_{j=1}^{p} \Phi^{-1}\left(u_{t-j}\right) \cdot \Phi^{-1}\left(v_{t-j}\right)+\gamma \cdot Z_{t}\right),
$$

where, as before, $\Lambda(\cdot)$ is the modified logistic transformation. The number of lags of cross products is chosen such that the fit of the model is best. $Z$ is a set of additional conditioning variables. If $Z$ is exogenous the distribution of the marginals must also be conditioned on it. In our case, however, it will be the dummies capturing the breakpoint in correlation and variance we detected with our procedure above. Dummies are included depending on the results obtained with the likelihood ratio tests given in Table 5.6.

The GARCH-variance are reported in Figure 5.2, whereas the conditional correlations are plotted in Figure 5.3. One can see that conditional correlation vary quite a lot over time, increasing after the "correlation" break. The evolution of the conditional correlation after the "volatility break" show a more shadowed picture: On one hand, it turns out that dependence decreases for some system leading to a "J-shape" evolution of the conditional correlations. Such a shape remains difficult to explain, even if it is confirmed by other studies using different techniques (Cappiello et al. 2005). 

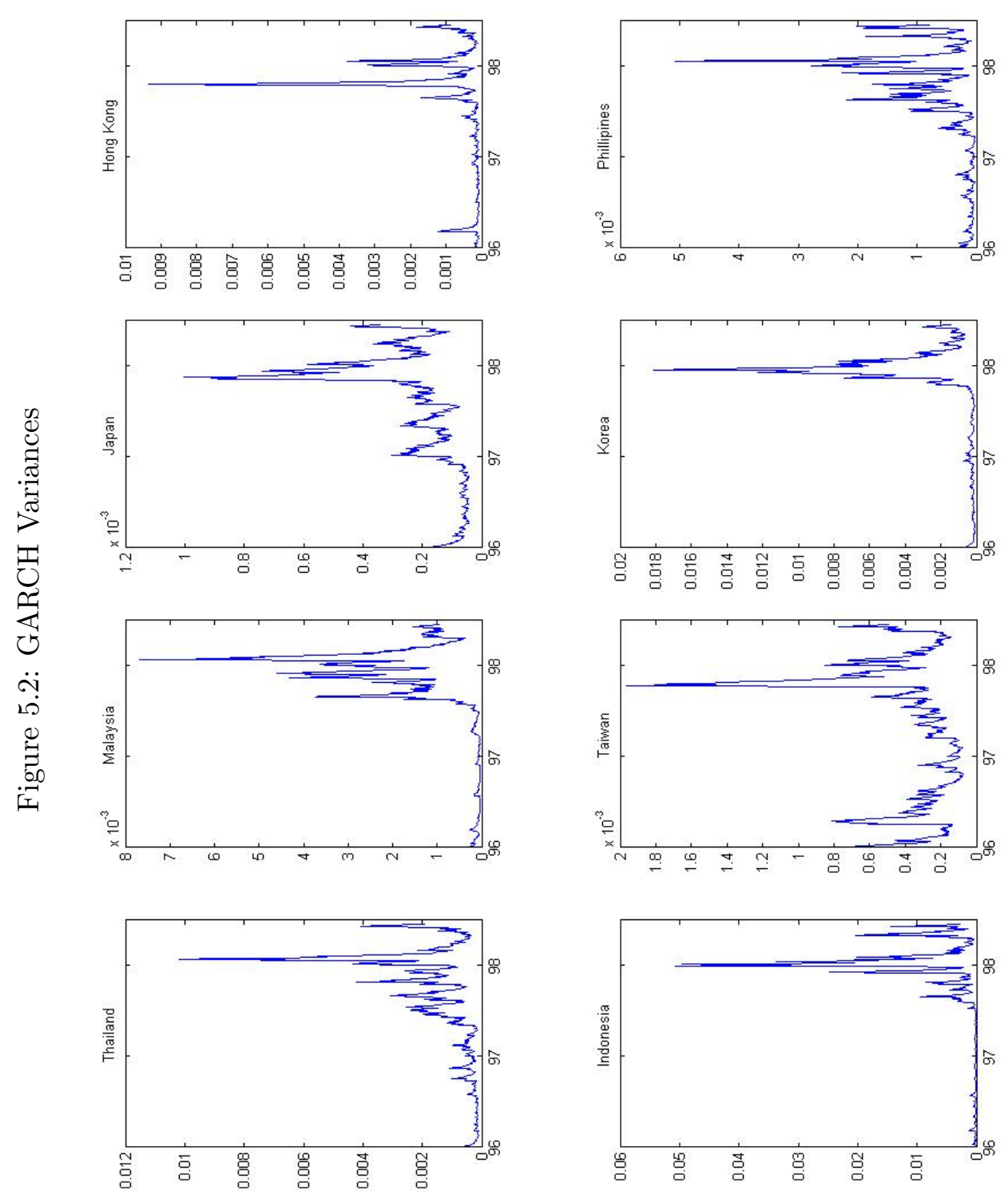

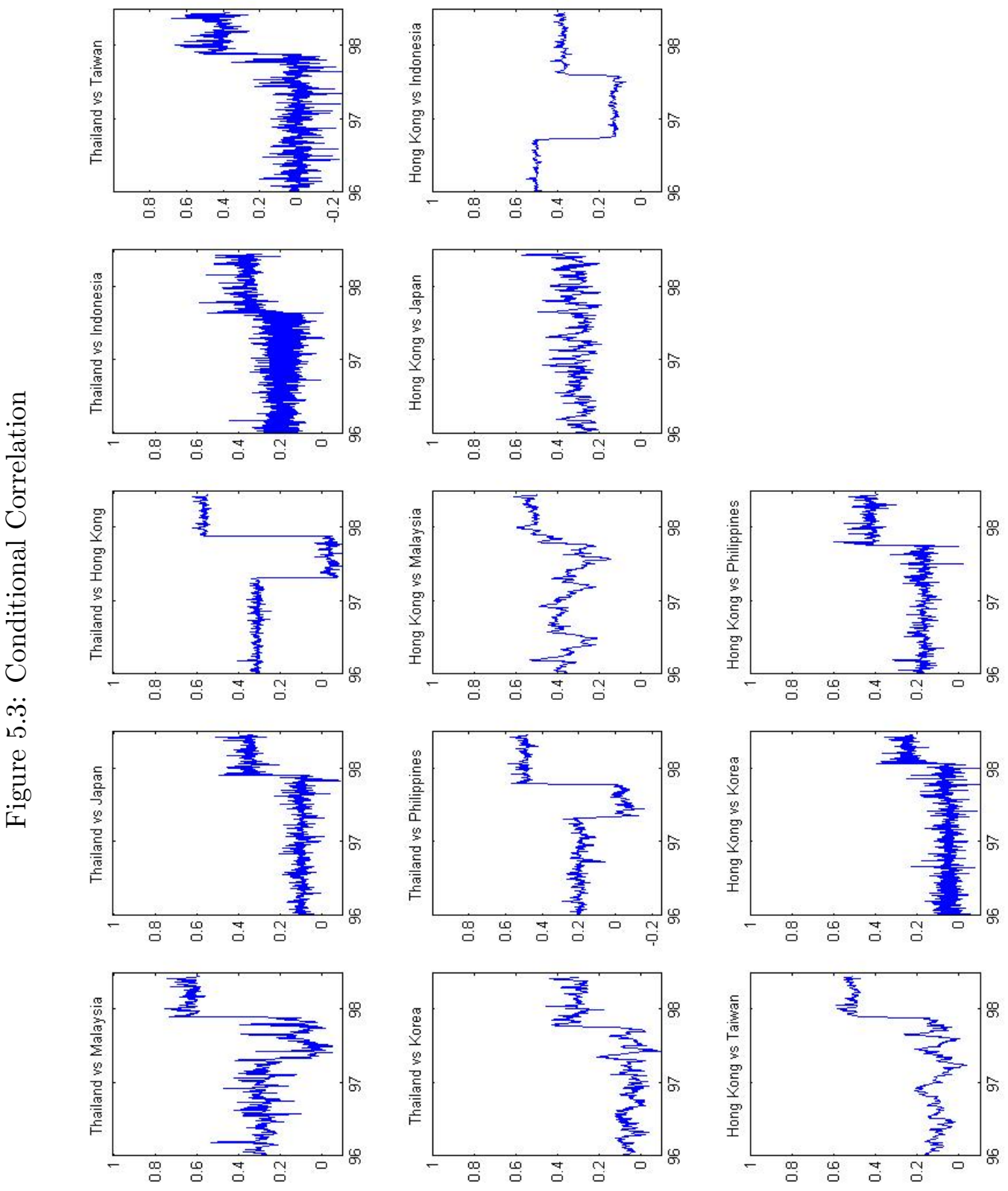
On the other hand, some pairs of countries exhibit a constant increase in the conditional correlation, stressing that dependence begins to increase with the volatility break. Nevertheless, Figures 5.2 and 5.3 support our previous conclusions that the break in variance generally precedes the one in correlation. This is thus further evidence that high volatility is not always concomitant to an increase in correlation.

\subsubsection{Robustness check}

The robustness of the previous results vis-à-vis the data and the estimation method of the conditional copula has been checked. We considered filtering the series only by AR models (instead of VAR), with or without GARCH filtering, and a fully-parametric estimation of the copula (i.e. specifying the marginal distributions parametrically), without finding different qualitative results. The qualitative results are also quite robust to the choice of copula. ${ }^{19}$ In some particular cases, applying a different type of copula appears to outperform the Gaussian copula. However, as the conclusions are not modified and the computing time explodes, we only report results for the Gaussian copula.

\subsection{Conclusion}

In this chapter we propose a new sequential procedure using time-varying copula to test for the presence of an increase in stock market dependence after a financial crisis, i.e. contagion process. We show that it is very important to consider simultaneously separate breaks in the volatility and dependence, otherwise the contagion test would be severely biased. In order to offer a more suitable approach, we develop a sequential algorithm, which allows for different breakpoints in the variance and the conditional correlation. Moreover, the proposed contagion test is a sequential "all-in-one" procedure which takes into account the uncertainty in the determination of the variance regime. The formal stability test is elaborated from the one proposed by Dias and Embrechts (2004) and a bootstrap procedure is implemented in order to tackle the distortion in the asymptotic distribution due to the presence of breakpoint in the nuisance parameters. Applied to the recent 1997 Asian crisis, the results produced by our sequential algorithm support that assuming the same break date for the variance and the conditional correlation is an erroneous assumption: Breaks in variances are generally preceding those in conditional correlation. Nevertheless, the Asian crisis turns out to have been characterized by a regional contagious transmission of the Thai shock.

\footnotetext{
${ }^{19}$ Further copulas considered are the Student, Clayton, Frank and Gumbel copulas and mixtures of them.
} 
Beyond the separate analysis of the effect of the volatility regime on the evolution of asset market dependence, tail-dependence may be also interesting as a complementary measure for contagion (Rodriguez 2007). Future research would include another step in the sequential procedure that would allow for lower tail dependence as well as changing types of dependence over time as studied by Rodriguez (2007). This could be performed using a more flexible copula model that additionally allows for conditional tail dependence. Even if such an analysis would bring a complementary insight on the tail dependence time path, it would not modify the previous results. 


\section{Dynamic stochastic copula models: Estimation, inference} and applications ${ }^{1}$

\subsection{Introduction}

Stochastic volatility (SV) models have attracted considerable interest in recent years, as they have been shown to offer a higher goodness-of-fit and flexibility than GARCH-type models when applied to financial time series, see e.g. Jacquier, Polson, and Rossi (1994), Danielsson (1998), Kim, Shephard, and Chib (1998), Carnero, Peña, and Ruiz (2004) for comparisons between the two model classes. With the same number, or less, of parameters the SV models gain additional flexibility through the use of stochastic latent variables that drive volatilities. Until quite recently, SV models have suffered from the difficult estimation problem, due to the fact that the evaluation of the likelihood function amounts to solving an integral of dimension equal to the sample size. Several methods have been proposed to circumvent this problem, see e.g. Broto and Ruiz (2004) for a survey. The proposed methods still require in most cases some computational effort, but thanks to increased computing power one can now estimate univariate SV models for typical sample sizes in the realm of seconds or even fractions of seconds. This makes them attractive to the applied econometrician and attenuates the comparative advantage of GARCH models in terms of computational simplicity.

Starting with Harvey, Ruiz, and Shephard (1994), multivariate SV models have become more popular in empirical finance to describe return volatilities and correlations, with direct applications e.g. to portfolio selection. Yu and Meyer (2006) are, to our knowledge, the first ones to propose a multivariate SV model with stochastic correlations, where the Fisher transform of the correlation follows a Gaussian AR(1) process. Asai and McAleer (2009) propose a model that is similar in structure to the DCC model of Engle (2002) but where the correlations are driven by a stochastic VAR(1)-type process. Amisano and Casarin (2007) suggest to introduce a Markov-switching process

\footnotetext{
${ }^{1}$ This chapter is based on Hafner and Manner (2008).
} 
to explain correlation dynamics in a multivariate SV framework. These model classes are very flexible, but they might fail to correctly describe the dependence between the series in situations where the dependence is nonlinear. If the multivariate return distribution conditional on the latent variables was elliptical, then correlations would be sufficient to describe dependence. However, one often observes non-elliptical distributions especially in finance, where the lower tail-dependence is usually much higher than upper tail-dependence.

A natural, and in fact the most general way to model non-linear dependencies is to use copulas, see e.g. the monograph of Joe (1997). A broad variety of copula models exists that allows to capture, for example, asymmetries in tail dependencies. One advantage of using copulas is the decoupling of marginal distributions from the dependence. This allows to first estimate the marginal processes, e.g. assuming univariate SV processes, and then in a second step the copula parameters. Furthermore, in situations where the marginal distribution cannot be assumed to be conditionally normal, the copula approach allows for the construction of new multivariate distributions by coupling the appropriate marginal distributions using a copula. A remarkable extension of copula theory has been made by Patton (2006b), who introduced the notion of a conditional copula and proposed a model for the evolution of time-varying dependence parameters. As an alternative to the Patton (2006b) model we propose a model where one or more of the copula parameters follows a transformation of a stochastic process, e.g. a Gaussian autoregressive process. As this is again a stochastic latent variable, it is in the same spirit as standard multivariate SV models. As a special case, we obtain a multivariate SV model with stochastic correlation by using a Gaussian copula. The use of other, in particular asymmetric copulas allows to better capture asymmetric tail dependencies. In this sense our model can be viewed as a generalization of standard multivariate SV models. However, as we will argue, estimation is straightforward due to the two step estimation. In particular, for estimation we use the efficient importance sampling (EIS) algorithm of Liesenfeld and Richard (2003) and Richard and Zhang (2007), and we extend it to the present model framework. We discuss specification tests such as parameter constancy and copula selection. In case of nonlinear transformations of the latent process, forecasting becomes a nontrivial issue, but we show that in most cases simple solutions can be found.

In the application part of the chapter we show that for two different bivariate stock index series, a stochastic copula model with SV marginals outperforms the Patton (2006b) model and a DCC model with $\operatorname{GARCH}(1,1)$ marginals, which is a natural competitor to our model and, due to its popularity, serves as a benchmark. This also holds if we replace the SV marginals by GARCH(1,1) to compare 
directly the dependence models, i.e. dynamic conditional correlation versus stochastic copula.

The remainder of the chapter is organized as follows. The next section introduces the model, discusses an estimation and inference method as well as the testing and forecasting problems. Section 6.3 discusses the application to bivariate stock index series, both at a daily and a weekly frequency and Section 6.4 concludes.

\subsection{Specification, estimation and diagnostics}

\subsubsection{The stochastic copula model}

Let $x$ and $y$ be two continuous random variables with joint distribution function $H(x, y)$ and marginal distribution $F$ and $G$, respectively. Then by Sklar's theorem there exists a unique copula $C$ such that

$$
H(x, y)=C(F(x), G(y))
$$

Thus by the probability integral transform a copula is a multivariate ${ }^{2}$ distribution function with uniform marginals and it fully captures the dependence between $x$ and $y$ irrespective of the marginal distributions.

Consider the bivariate time series process $\left(u_{t}, v_{t}\right)$ for $t=1, \ldots, T$ with distribution function given by the following time-varying copula model

$$
\left(u_{t}, v_{t}\right) \sim C\left(u, v \mid \theta_{t}\right)
$$

where $\theta_{t} \in \Theta \subset \mathbb{R}^{K}$ is a random parameter vector of the copula function. In this chapter we only consider copulas with one parameter, so that $K=1$. We assume that $\theta_{t}$ is driven by an unobserved stochastic process $\lambda_{t}$ such that $\theta_{t}=\Psi\left(\lambda_{t}\right)$, where $\Psi: \mathbb{R} \rightarrow \Theta$ is an appropriate transformation to ensure that the copula parameter remains in its domain and whose functional form depends on the choice of copula. The underlying dependence parameter $\lambda_{t}$, which is unobserved, is assumed to follow a Gaussian autoregressive process of order one,

$$
\lambda_{t}=\alpha+\beta \lambda_{t-1}+\nu \varepsilon_{t},
$$

\footnotetext{
${ }^{2}$ We only consider bivariate copulas in this chapter, but most methods can in principle be extended to the multivariate case, see Section 6.2.8.
} 
where $\varepsilon_{t}$ is an i.i.d. $N(0,1)$ innovation. In principle, higher order autoregressive models could be considered, but estimation would become very difficult and in all applications we found that $\operatorname{AR}(1)$ sufficiently describes persistence and the autocorrelation structure of the dependence parameter. Furthermore, the first order autoregressive model allows for the state space representation of the model we describe below. We assume that the latent process is strictly stationary, i.e. $|\beta|<1$. For identification reasons we also assume that the scale parameter of the innovations, $\nu$, is positive. Note that we ignore specification of the marginals for the moment, so we assume that the observed variables have a $U(0,1)$ distribution or, alternatively, we assume perfect knowledge of the marginals. We will discuss possibilities on how to deal with the marginals below.

The copulas used in this chapter are the most popular one-parameter families, the Gaussian, Frank, Clayton, Gumbel and rotated versions of the Clayton and Gumbel copulas. The fact that the parameter of the Frank copula lies in $(-\infty, \infty) \backslash 0$ makes it particularly attractive in our case, since we can chose $\Psi(x)=x$, which implies that the time-varying parameter $\theta_{t}$ itself follows a Gaussian AR(1) process. For the Gaussian copula we will we use the inverse Fisher transform $\Psi(x)=(\exp (2 x)-1) /(\exp (2 x)+1)$ to ensure that its parameter lies in $(-1,1)$ at all times. Note that this choice is natural as the Fisher transform is the variance stabilizing transformation for the correlation coefficient (see van der Vaart, 1998). For the Clayton copula it holds that $\theta \in(0, \infty)$. This suggests the transformation $\Psi(x)=\exp (x)$ and implies that the dependence parameter has a $\log$-normal distribution. Finally, for the Gumbel copula $\theta \in[1, \infty)$, so an obvious choice for the transformation is $\Psi(x)=\exp (x)+1$, which again implies log-normality of the dependence parameter.

The above model has a nonlinear state space representation with observation equation $\left(u_{t}, v_{t}\right) \mid$ $\lambda_{t} \sim C\left(u, v \mid \Psi\left(\lambda_{t}\right)\right)$ and transition equation given by (6.3). A straightforward extension is possible when the marginals are unknown and included in the model. Hence, the estimation procedure used in this chapter can be interpreted as a nonlinear filtering algorithm and is linked to the extensive literature on nonlinear filtering, see e.g. Doucet, de Freitas, and Gordon (2001). In particular, Durbin and Koopman $(2000,2001)$ discuss how importance sampling can be used to estimate timeseries models in state space form. In the following we describe how a particular importance sampling technique can be adapted to estimate our model.

\subsubsection{Estimation}

We are interested in estimating the parameter vector $\omega=(\alpha, \beta, \nu)$. Denote $U=\left\{u_{t}\right\}_{t=1}^{T}, V=\left\{v_{t}\right\}_{t=1}^{T}$ and $\Lambda=\left\{\lambda_{t}\right\}_{t=1}^{T}$ and let $f(U, V, \Lambda ; \omega)$ be the joint density of the observable variables $(U, V)$ and the 
latent process $\Lambda$. Then the likelihood function of the parameter vector $\omega$ is

$$
L(\omega ; U, V)=\int f(U, V, \Lambda ; \omega) d \Lambda
$$

If now $U_{t}=\left\{u_{\tau}\right\}_{\tau=1}^{t}$, and similarly for $V_{t}$ and $\Lambda_{t}$, we can factorize the integrand of this likelihood function into a sequence of conditional densities as follows.

$$
L(\omega ; U, V)=\int \prod_{t=1}^{T} f\left(u_{t}, v_{t}, \lambda_{t} \mid U_{t-1}, V_{t-1}, \Lambda_{t-1}, \omega\right) d \Lambda .
$$

Furthermore the joint density $f\left(u_{t}, v_{t}, \lambda_{t} \mid U_{t-1}, V_{t-1}, \Lambda_{t-1}, \omega\right)$ can be factorized into the copula density $c\left(u_{t}, v_{t} \mid \lambda_{t}, U_{t-1}, V_{t-1}, \omega\right)$ times the conditional density of $\lambda_{t}$ given $\left(U_{t-1}, V_{t-1}, \Lambda_{t-1}\right)$, which is $p\left(\lambda_{t} \mid U_{t-1}, V_{t-1}, \Lambda_{t-1}, \omega\right)$. Since $p$ does not depend on the past observable variables $\left(U_{t-1}, V_{t-1}\right)$ they can be omitted for the sake of notation. This gives the following likelihood function for $\omega$

$$
L(\omega ; U, V)=\int \prod_{t=1}^{T} c\left(u_{t}, v_{t} \mid \lambda_{t}, U_{t-1}, V_{t-1}, \omega\right) p\left(\lambda_{t} \mid \Lambda_{t-1}, \omega\right) d \Lambda
$$

This integral is $T$-dimensional and cannot be evaluated by analytical or numerical methods even for moderate sample sizes. However, it can be evaluated by simulation. In principle, one could simulate a large number $N$ of trajectories $\left\{\tilde{\lambda}_{t}^{(i)}(\omega)\right\}_{t=1}^{T}$ from $p$, which we call the natural sampler, and evaluate the likelihood function by

$$
\hat{L}_{N}(\omega ; U, V)=\frac{1}{N} \sum_{i=1}^{N}\left[\prod_{t=1}^{T} c\left(u_{t}, v_{t} \mid \tilde{\lambda}_{t}^{(i)}(\omega), U_{t-1}, V_{t-1}, \omega\right)\right] .
$$

However, as mentioned in Danielsson and Richard (1993) and Liesenfeld and Richard (2003) this estimator, labeled the "natural MC estimate", is very inefficient for reasonably large sample sizes. This results from the fact that the trajectories $\left\{\tilde{\lambda}_{t}^{(i)}(\omega)\right\}_{t=1}^{T}$ are sampled independently of the observed variables $U$ and $V$ and thus do not make any use of the information available in the data.

A technique to handle such problems, which is proposed in Liesenfeld and Richard (2003) and Richard and Zhang (2007), is called efficient importance sampling (EIS). The main idea of EIS is to make use of the information on $\Lambda$ contained in the observable variables $U$ and $V$ to construct a new sampler that exploits this information. Denote a sequence of this auxiliary sampler by $\left\{m\left(\lambda_{t} \mid \Lambda_{t-1}, a_{t}\right)\right\}_{t=1}^{T}$ indexed by the auxiliary parameters $a_{t}$, which need to be estimated. The 
likelihood function can be rewritten as

$$
L(\omega ; U, V)=\int \prod_{t=1}^{T}\left[\frac{f\left(u_{t}, v_{t}, \lambda_{t} \mid U_{t-1}, V_{t-1}, \Lambda_{t-1}, \omega\right)}{m\left(\lambda_{t} \mid \Lambda_{t-1}, a_{t}\right)}\right] \prod_{t=1}^{T} m\left(\lambda_{t} \mid \Lambda_{t-1}, a_{t}\right) d \Lambda,
$$

which can be evaluated by using $N$ trajectories $\left\{\tilde{\lambda}_{t}^{(i)}\left(a_{t}\right)\right\}_{t=1}^{T}$ drawn from the importance sampler $m$ by

$$
\tilde{L}_{N}(\omega ; U, V)=\frac{1}{N} \sum_{i=1}^{N}\left(\prod_{t=1}^{T}\left[\frac{f\left(u_{t}, v_{t}, \tilde{\lambda}_{t}^{(i)}\left(a_{t}\right) \mid U_{t-1}, V_{t-1}, \tilde{\Lambda}_{t-1}^{(i)}\left(a_{t-1}\right), \omega\right)}{m\left(\tilde{\lambda}_{t}^{(i)}\left(a_{t}\right) \mid \tilde{\Lambda}_{t-1}^{(i)}\left(a_{t-1}\right), a_{t}\right)}\right]\right)
$$

The challenge of EIS is to find a function $m$ and a sequence of auxiliary parameters $\left\{a_{t}\right\}_{t=1}^{T}$ to provide a good match between the numerator and the denominator in (6.9) in order to reduce the MC sampling variance of $\tilde{L}_{N}$ as much as possible. The auxiliary parameters need to be estimated, which must be done for each period $t$ due to the high dimensionality of the problem. A good match between $f$ and $m$ is not possible period by period since the integral of $f$ with respect to $\lambda_{t}$ depends on $\Lambda_{t-1}$, while that of $m$ is equal to one. Therefore we need a functional approximation $k\left(\Lambda_{t} ; a_{t}\right)$ for $f$ that is analytically integrable with respect to $\lambda_{t}$ such that

$$
m\left(\lambda_{t} \mid \Lambda_{t-1}, a_{t}\right)=\frac{k\left(\Lambda_{t} ; a_{t}\right)}{\chi\left(\Lambda_{t-1} ; a_{t}\right)}
$$

where $\chi$ denotes the integral of $k$ with respect to $\lambda_{t}$. Note that we must account for the function $\chi$ when matching $f$ and $k$, but since $\chi$ does not depend on $\lambda_{t}$ it can be transferred back into the subproblem for period $t-1$. Then for each period $t$ given a value for $\omega$ the following minimization problem must be solved

$$
\begin{aligned}
\hat{a}_{t}=\arg \min _{a_{t}} \Sigma_{i=1}^{N} & \left(\log \left[f\left(u_{t}, v_{t}, \tilde{\lambda}_{t}^{(i)}(\omega) \mid U_{t-1}, V_{t-1}, \tilde{\Lambda}_{t-1}^{(i)}(\omega), \omega\right) \cdot \chi\left(\tilde{\Lambda}_{t}^{(i)}(\omega) ; \hat{a}_{t+1}\right)\right]\right. \\
& \left.-c_{t}-\log k\left(\tilde{\Lambda}_{t}^{(i)}(\omega) ; a_{t}\right)\right)^{2}
\end{aligned}
$$

for $t=T, \ldots, 1$ and $\chi\left(\Lambda_{T} ; a_{T+1}\right) \equiv 1$. Since the trajectories of the underlying dependence process are drawn from the natural sampler, one should iterate this procedure and use draws $\left\{\tilde{\lambda}_{t}^{(i)}\left(\hat{a}_{t}\right)\right\}_{t=1}^{T}$ from the importance sampler in the next iteration until convergence of $\hat{a}_{t}$ to fixed values. This requires the use of Common Random Numbers (CRNs) at each iteration. Furthermore, if $k\left(\Lambda ; a_{t}\right)$ is chosen within the exponential family the least squares problem in (6.11) becomes linear. The likelihood function is then evaluated by substituting the estimated sequence $\left\{\hat{a}_{t}\right\}_{t=1}^{T}$ and $N$ draws from the 
importance sampler $m$ into (6.9).

Liesenfeld and Richard (2003) suggest the following decomposition of $k$

$$
k\left(\Lambda_{t} ; a_{t}\right)=p\left(\lambda_{t} \mid \lambda_{t-1}, \omega\right) \zeta\left(\lambda_{t}, a_{t}\right),
$$

with $\zeta\left(\lambda_{t}, a_{t}\right)$ a Gaussian kernel. This decomposition further simplifies the least squares problem and the functional form of the likelihood as the natural sampler $p$ now cancels out. The choice $\zeta\left(\lambda_{t}, a_{t}\right)=\exp \left(a_{1, t} \lambda_{t}+a_{2, t} \lambda_{t}^{2}\right)$ makes the least squares problem in (6.11) linear and thus greatly reduces the computational burden of the procedure. For non-Gaussian latent processes, however, the importance sampler should be chosen in a different way, which will most likely not result in a linear least squares problem. Given this choice the mean and variance of the importance sampler $m$, which depend on $a_{t}=\left(a_{1, t}, a_{2, t}\right)$, are

$$
\mu_{t}=\sigma_{t}^{2}\left(\frac{\alpha+\beta \lambda_{t-1}}{\nu^{2}}+a_{1, t}\right), \quad \sigma_{t}^{2}=\frac{\nu^{2}}{1-2 \nu^{2} a_{2, t}}
$$

The functional forms of $p, k$ and $\chi$ are given by Liesenfeld and Richard (2003). For a given choice of the copula, EIS can be implemented as follows.

1. Draw $N$ trajectories $\left\{\tilde{\lambda}_{t}^{(i)}(\omega)\right\}_{t=1}^{T}$ from the natural sampler $p$.

2. For $t=T, \ldots, 1$ solve the back-recursive least-squares regression problem

$$
\begin{aligned}
& \log c\left(u_{t}, v_{t} \mid \theta_{t}(\omega)\right)+\log \chi\left(\tilde{\lambda}_{t}^{(i)}(\omega) ; \hat{a}_{t+1}\right) \\
& =c_{t}+a_{1, t} \tilde{\lambda}_{t}^{(i)}(\omega)+a_{2, t}\left[\tilde{\lambda}_{t}^{(i)}(\omega)\right]^{2}+\eta_{t}^{(i)}
\end{aligned}
$$

with $c_{t}$ and $\eta_{t}^{(i)}$ the regression constant and error term, respectively.

3. Draw $N$ trajectories $\left\{\tilde{\lambda}_{t}^{(i)}\left(\hat{a}_{t}\right)\right\}_{t=1}^{T}$ from the importance sampler $m$ and solve the least-squares problem in Step 2 again. Iterate Steps 2 and 3 this until convergence of $\left\{\hat{a}_{t}\right\}_{t=1}^{T}$.

4. Draw $N$ trajectories $\left\{\tilde{\lambda}_{t}^{(i)}\left(\hat{a}_{t}\right)\right\}_{t=1}^{T}$ from the importance sampler $m$ and evaluate the likelihood function given in (6.9). 
The maximum likelihood estimator of the parameter vector $\omega$ is then obtained by maximizing the EIS likelihood function. In order to ensure its smoothness, the same CRNs are used for every evaluation of the likelihood function. Note that more than five iterations are not necessary in most cases. Concerning the number of trajectories $N$, a choice between 100 and 200 seems to be sufficient to keep the Monte Carlo variation small.

\subsubsection{Including the marginals: One Step vs. Two Step Estimation}

So far we have ignored that the marginals must also be modeled and estimated. Generally speaking this depends highly on the type of data one wants to model. Since in this chapter we are mainly focusing on financial data observed with a daily or weekly frequency, in particular stock market returns, it is crucial to properly model the time-varying volatility and leptokurtosis. However, within this chapter we limit ourselves to the specific case that each variable only depends on its own past, but not on the past of the other variable, and that there is only instantaneous causality between the variables. Chen and Fan (2006b) are a bit more general by allowing the conditional mean to depend on the past of both variables, whereas in Patton (2006a) general conditioning variables are allowed for and it is noted that both marginal distributions and the copula must be conditioned on the same information. As a consequence all densities should be defined as conditional densities in that case.

A natural model for the $i$-th stock market returns $r_{i t}$ for $t=1, \ldots, T$ is the stochastic volatility (SV) model proposed by Clark (1973) and Taylor (1986). In its simplest form it can be written as

$$
\begin{aligned}
& r_{i t}=\exp \left(h_{i t} / 2\right) \varepsilon_{i t} \\
& h_{i t}=\alpha_{i}+\beta_{i} h_{i t-1}+\nu_{i} \eta_{i t},
\end{aligned}
$$

where $\varepsilon_{i t}$ and $\eta_{i t}$ are mutually independent i.i.d. normal random variables with mean zero and variance one that are also uncorrelated with the innovations driving the dependence process. Estimation may be done using EIS as described in Liesenfeld and Richard (2003).

An alternative to the SV model is the large class of GARCH models, introduced by Engle (1982) and Bollerslev (1986). The standard GARCH(1,1) model is given by

$$
\begin{aligned}
r_{i t} & =\sqrt{h_{i t}} \varepsilon_{i t} \\
h_{i t} & =\alpha_{i}+\gamma_{i} \varepsilon_{i t-1}^{2}+\beta_{i} h_{i t-1} .
\end{aligned}
$$

As mentioned in the introduction, it is easily possible to relax the assumption of conditional normality 
of the marginal distributions. For example $\varepsilon_{i t}$ may come from a t-distribution with different degrees of freedom for each series and the dependence may still be modeled using a Gaussian copula. This is not possible using generic multivariate distributions such as the multivariate Student t. Note that the joint model allows a nonlinear state space representation. Denote by $\Phi(\cdot)$ the standard normal cdf. Then the observation equation is given by

$$
\left(r_{i t}, r_{j t}\right) \mid \lambda_{t}, h_{i t}, h_{j t} \sim C\left(\Phi\left(\varepsilon_{i t}\right), \Phi\left(\varepsilon_{j t}\right)\right)
$$

where in the SV model $\varepsilon_{i t}=\exp \left(-h_{i t} / 2\right) r_{i t}$ and the transition equation is given by (6.15), while in the GARCH model, $\varepsilon_{i t}=r_{i t} / \sqrt{h_{i t}}$ and the transition equation is given by (6.16). The state variables $h_{1 t}$ and $h_{2 t}$ of the marginal models do not depend on the states of the process $\lambda_{t}$ driving the dependence, which justifies the sequential estimation of the marginals and the copula we describe below.

For either choice of the model for the marginals, denote the parameter vector for component $i$ by $\delta_{i}$ for $i=1,2$ and $\omega$ for the copula. Assume we observe the processes $X=\left\{x_{t}\right\}_{t=1}^{T}$ and $Y=\left\{y_{t}\right\}_{t=1}^{T}$ with marginal distributions $F\left(X ; \delta_{1}\right)$ and $G\left(Y ; \delta_{2}\right)$. Then, as a consequence of Sklar's theorem, the joint log-likelihood function can be decomposed into the marginal likelihood and the copula likelihood,

$$
\mathscr{L}\left(\delta_{1}, \delta_{2}, \omega ; X, Y\right)=\mathscr{L}_{X}\left(\delta_{1} ; X\right)+\mathscr{L}_{Y}\left(\delta_{2} ; Y\right)+\mathscr{L}_{C}\left(\omega ; F\left(X ; \delta_{1}\right), G\left(Y ; \delta_{2}\right)\right)
$$

In principle, (6.17) can be estimated w.r.t. all parameters to give a fully efficient maximum likelihood estimate whose covariance matrix is given by the inverse of the Fisher information matrix. However, joint estimation of all parameters is computationally very expensive in our situation, in particular when SV models are chosen for the margins, because in that case all three components of (6.17) would need to be evaluated jointly by an algorithm such as EIS. In some cases it may not even be possible to reach convergence at all. To solve this problem, one may maximize the marginal log-likelihood functions in a first step, and then maximize the copula log-likelihood conditional on the estimated marginals. This method has been labeled the inference function for margins (IFM) estimator by Joe $(1997,2005)$. Under weak regularity conditions, this estimator is consistent, although not fully efficient. Standard errors of the two step estimator $\hat{\omega}$ can be obtained as follows. Let us denote the full parameter vector as $\vartheta=\left(\delta_{1}^{\prime}, \delta_{2}^{\prime}, \omega^{\prime}\right)^{\prime}$ and let $\psi(X, Y)=\left(\partial \mathscr{L}_{X} / \partial \delta_{1}^{\prime}, \partial \mathscr{L}_{Y} / \partial \delta_{2}^{\prime}, \partial \mathscr{L}_{\mathscr{C}} / \partial \omega^{\prime}\right)^{\prime}$ denote the inference functions. Furthermore, let us denote $D=E\left[\partial \psi(X, Y) / \partial \vartheta^{\prime}\right]$ and $M=E\left[\psi(X, Y) \psi(X, Y)^{\prime}\right]$. 
Then, as shown by Joe $(2005), \sqrt{T}(\hat{\vartheta}-\vartheta) \rightarrow_{d} N(0, V)$, where

$$
V=D^{-1} M\left(D^{-1}\right)^{\prime}
$$

We will use this result for inference in our applications.

Note that the possibility of two-step estimation is not exclusive to copula based models, but has also been proposed for the DCC model by Engle (2002) and for the model by Asai and McAleer (2009). However, these procedures are in fact equivalent to using the copula decomposition of the log-likelihood function with the Gaussian copula.

\subsubsection{Estimating the underlying process}

Even though the parameters of the underlying process are of interest themselves, it is of crucial importance to obtain an estimate of the sequence of the underlying latent state variable $\lambda_{t}$ and of the function $\Psi\left(\lambda_{t}\right)$. To this end we need to compute the conditional expectation of $\Psi\left(\lambda_{t}\right)$ given the information on the past observable variables $U_{t-1}$ and $V_{t-1}$. This is known as the filtered (or predicted) estimates of $\Psi\left(\lambda_{t}\right)$, which we denote $\Psi\left(\hat{\lambda}_{t \mid t-1}\right)$ and is given by

$$
E\left[\Psi\left(\lambda_{t}\right) \mid U_{t-1}, V_{t-1}\right]=\frac{\int \Psi\left(\lambda_{t}\right) p\left(\lambda_{t} \mid \Lambda_{t-1}, \omega\right) f\left(U_{t-1}, V_{t-1}, \Lambda_{t-1} ; \omega\right) d \Lambda_{t}}{\int f\left(U_{t-1}, V_{t-1}, \Lambda_{t-1} ; \omega\right) d \Lambda_{t-1}}
$$

Using the estimated parameters $\hat{\omega}$ the denominator is simply the likelihood function using the first $t-1$ observations, which is $L\left(\omega ; U_{t-1}, V_{t-1}\right)$. The numerator can be evaluated by

$$
\frac{1}{N} \sum_{i=1}^{N}\left(\Psi\left[\tilde{\lambda}_{t}^{(i)}(\hat{\omega})\right] \prod_{t=1}^{t-1}\left[\frac{f\left(u_{\tau}, v_{\tau}, \tilde{\lambda}_{t}^{(i)}\left(\hat{a}_{\tau}\right) \mid U_{\tau-1}, V_{\tau-1}, \tilde{\Lambda}_{\tau-1}^{(i)}\left(\hat{a}_{\tau-1}\right), \hat{\omega}\right)}{m\left(\tilde{\lambda}_{\tau}^{(i)}\left(\hat{a}_{\tau}\right) \mid \tilde{\Lambda}_{\tau-1}^{(i)}\left(\hat{a}_{\tau-1}\right), \hat{a}_{\tau}\right)}\right]\right)
$$

with $\left\{\tilde{\lambda}_{\tau}^{(i)}\left(\hat{a}_{\tau}\right)\right\}_{\tau=1}^{t-1}$ a trajectory from the EIS sampler for $L\left(\omega ; U_{t-1}, V_{t-1}\right)$ and $\tilde{\lambda}_{t}^{(i)}(\hat{\omega})$ a draw from the natural sampler $p\left(\lambda_{t} \mid \tilde{\Lambda}_{t-1}^{(i)}, \hat{\omega}\right)$. As before CRN's should be used to evaluate the numerator and the denominator. The integral in (6.19) must be evaluated for each $t=1, \ldots, T$. In addition it needs to be mentioned that the number $N$ of trajectories from the importance sampler must be a lot higher than for estimation purposes in order to ensure numerical stability at each evaluation of the likelihood function. We recommend a number of about 500, which means that evaluation of a filtered path for 2500 observations requires about 2-3 hours of computation on an Intel dual core processor.

An alternative to the computationally expensive method of filtering is to make use of the mechanics of EIS to obtain smoothed estimates of the latent process, i.e. to estimate each $\Psi\left(\lambda_{t}\right)$ using the 
complete information available at time T. As mentioned above, EIS exploits all the information available in the data to produce efficient samples of the underlying process $\left\{\lambda_{t}\right\}_{t=1}^{T}$. Thus as a byproduct of EIS when evaluating the likelihood function we obtain the smoothed estimate of $\left\{\Psi\left(\lambda_{t}\right)\right\}_{t=1}^{T}$ by

$$
\Psi\left(\hat{\lambda}_{t \mid T}\right)=\frac{1}{N} \sum_{i=1}^{N} \Psi\left(\tilde{\lambda}_{t}^{(i)}\left(\hat{a}_{t}\right)\right) \quad \forall t=1, \ldots, T .
$$

Using both simulated and real data, we obtained smoothed estimates of $\left\{\Psi\left(\lambda_{t}\right)\right\}_{t=1}^{T}$ that were very close to the filtered and true (in case of simulated data) paths of the underlying process. Furthermore, the smoothed estimates are much less noisy, due to the fact that they are calculated as an average, but also since they make efficient use of the complete information contained in the sample at each $\Psi\left(\hat{\lambda}_{t \mid T}\right)$. Furthermore, it is computationally much cheaper and requires only a small $N$.

A third option is to calculate the smoothed estimate of $\Psi\left(\lambda_{t}\right)$ using only the information available at time $t$, i.e. $U_{t}$ and $V_{t}$. This estimate, which may be called the 'updated' estimate shall be denoted by $\Psi\left(\hat{\lambda}_{t \mid t}\right)$. In order to understand how filtered estimates are obtained one should be aware that these are in fact the one-step forecasts of the updated state variable accounting for the nonlinearity of the transformation $\Psi$.

Since the main objective of time-varying correlation models is to estimate the correlation path over time, we conduct a small simulation study to see how competing methods for estimating time-varying correlation compare in the sense of being closer to the true correlation path. We draw a sample of size 1000 from a Gaussian copula model with a variety of underlying correlation dynamics. Four competing models are fit, the estimates for time-varying correlations are retrieved and mean square errors (MSE) are computed. The models are a constant copula, the stochastic copula autoregressive (SCAR) model $^{3}$ and the DCC GARCH model of Engle (2002), where correlation is described by

$$
\begin{aligned}
\rho_{i, j, t} & =\frac{q_{i, j, t}}{\sqrt{q_{i, i, t} q_{j, j, t}}} \\
q_{i, j, t} & =\bar{\rho}_{i, j}+\alpha\left(\varepsilon_{i, t-1} \varepsilon_{j, t-1}-\bar{\rho}_{i, j}\right)+\beta\left(q_{i, j, t-1}-\bar{\rho}_{i, j}\right),
\end{aligned}
$$

with $\bar{\rho}$ the unconditional sample correlation and $\varepsilon_{i, t}$ the standardized GARCH residual for variable $i$ at time $t$. Note that in our simulation study we do not consider volatility dynamics, but only the model for correlation. The fourth model we consider is the conditional copula specification from Patton (2006b), which is similar to the DCC model, but does not assume any marginal distribution.

\footnotetext{
${ }^{3}$ The correlation estimate is the smoothed correlation path.
} 
The conditional correlation in this model is given by

$$
\rho_{t}=\Psi\left(\alpha+\beta \rho_{t-1}+\nu \frac{1}{10} \sum_{j=1}^{10} \Phi^{-1}\left(u_{t-j}\right) \Phi^{-1}\left(v_{t-j}\right)\right)
$$

where $\Psi$ is chosen to be the inverse Fisher transform. The correlations follow several processes, both deterministic and stochastic, which are:

1. Constant: $\rho_{t}=0.5$

2. Jump: $\rho_{t}=0.2+0.5 I_{t>500}$

3. Sine: $\rho_{t}=0.5+0.4 \cos (2 \pi t / 200)$

4. Ramp: $\rho_{t}=\bmod (t / 200)$

5. DCC: Correlation generated by (6.22) with $\bar{\rho}=0.7, \alpha=0.04$ and $\beta=0.95$

6. $\operatorname{AR}(1): \lambda_{t}=0.03+0.97 \lambda_{t-1}+0.1 \varepsilon_{t}$

7. Noise: $\lambda_{t}=0.3+0.1 \varepsilon_{t}$

8. Random Walk (RW): $\lambda_{t}=\lambda_{t-1}+0.01 \varepsilon_{t}$

9. $\operatorname{ARMA}(2,2): \lambda_{t}=0.01+0.65 \lambda_{t-1}+0.3 \lambda_{t-2}+0.1 \varepsilon_{t}+0.05 \varepsilon_{t-1}+0.03 \varepsilon_{t-2}$

where $\varepsilon_{t}$ is a $N(0,1)$ random variable. For the DCC model the parameters were those estimated for the Dow Jones-NASDAQ data in the empirical application. For processes 6 to 9 we consider two transformations to keep correlation between -1 and 1 at all times: The first one is the inverse Fisher transform, whereas the second one is $\rho_{t}=\lambda_{t} / \sup _{t}\left|\lambda_{t}\right|$. The number of trajectories $N$ in the EIS sampler is chosen to be 200. We repeat the simulation 1000 times for the constant copula and the DCC model and only 100 times for the SCAR and Patton models due to computational complexity. We report the average of the mean squared distance between the true and the estimated correlation path. Results, which are reported in Table 6.1, show that the SCAR model clearly outperforms its competitors both under deterministic and stochastic correlation dynamics and regardless of the transformation. Not surprisingly, all models do worse when they are misspecified. The DCC only performs better when it is also the data generating process, which is not surprising as in that case the innovations driving the correlation are known. Still, the SCAR model does well and outperforms the specification of Patton (2006b). Note that in Asai and McAleer (2009) a similar simulation study is 
performed using DGP's very close to 1-4. In terms of estimating $\rho_{t}$ their dynamic correlation model is found to perform worse than the DCC, even though the volatilities in their DPG follow an SV model, which should strongly favor their SV based model over the (misspecified) DCC-GARCH.

\subsubsection{Testing}

We now consider two hypotheses that may be of interest in empirical modeling using the stochastic copula model. The first is whether the dependence parameter is actually time-varying. Formally, the null hypothesis can be written as

$$
H_{0}: \theta_{t}=\bar{\theta}, \quad \forall \quad t=1, \ldots, T
$$

where $\bar{\theta}$ is the time-constant copula parameter. In terms of our model parameters in (6.3) this null hypothesis can also be stated as

$$
H_{0}: \nu=0
$$

We test this hypothesis with a simple likelihood ratio test. Let $L L_{r e s}$ be the log-likelihood of the model under $H_{0}$ and $L L_{u r}$ the log-likelihood of the unrestricted model. The test statistic is

$$
L R_{C}=-2\left(L L_{r e s}-L L_{u r}\right)
$$

Since $\beta$ is unidentified under the null and furthermore $\nu$ is on the boundary of the parameter space the asymptotic distribution of $L R_{C}$ is non-standard. However, we can obtain approximate critical values by simulation. These critical values, which are approximately $4.65,6.44$ and 9.99 , at $10 \%$, $5 \%$ and $1 \%$ level of significance, respectively, are close to those of a $\chi^{2}$ distribution with 2 degrees of freedom. The second important hypothesis is that of the correct choice of the copula. The most simple, but still very reliable way of choosing the best fitting copula is to compare the values of the log-likelihood at the parameter estimates or the Akaike Information Criterion (AIC) when the competing models have different numbers of parameters. Although this works quite well, we have no guarantee that the model chosen in such a way fits the data well. There are various approaches to the problem of goodness-of-fit (GoF) testing in copulas, see e.g. Genest and Rivest (1993), Genest et al. (2006), Chen and Fan (2006a), Junker, Szimayer, and Wagner (2006), Patton (2006b), or for a comparison of some tests Genest, Rémillard, and Beaudoin (2009). Let $C_{i}\left(u_{t}, v_{t}, \hat{\theta}_{t}\right)$ be our candidate 
Table 6.1: Average MSEs for correlation estimates

\begin{tabular}{lccccccccc}
\hline \hline Model/DGP & constant & jump & Sine & ramp & DCC & AR(1) & Noise & RW & ARMA $(2,2)$ \\
\hline SCAR & 0.0005 & 0.0066 & 0.0130 & 0.0261 & 0.0041 & 0.0087 & 0.0104 & 0.0081 & 0.0556 \\
DCC & 0.0010 & 0.0134 & 0.0318 & 0.0458 & 0.0012 & 0.0209 & 0.0104 & 0.0090 & 0.1096 \\
Patton & 0.0019 & 0.0416 & 0.0530 & 0.0589 & 0.0062 & 0.0215 & 0.0134 & 0.0119 & 0.1193 \\
Constant & 0.0004 & 0.0631 & 0.0806 & 0.0838 & 0.0111 & 0.0356 & 0.0093 & 0.0154 & 0.1909 \\
\hline Alternative transformation & & & & & & & & \\
SCAR & - & - & - & - & - & 0.0173 & 0.0267 & 0.0128 & 0.0402 \\
DCC & - & - & - & - & - & 0.0282 & 0.0268 & 0.0247 & 0.0714 \\
Patton & - & - & - & - & - & 0.0315 & 0.0277 & 0.0437 & 0.0701 \\
Constant & - & - & - & - & - & 0.0350 & 0.0259 & 0.0972 & 0.1025 \\
\hline \hline
\end{tabular}

Note: Average mean square errors of time-varying correlation estimates. In the top panel the underlying process $\lambda_{t}$ is re-scaled by the inverse Fisher transform, whereas in the bottom panel the transformation $\rho_{t}=\lambda_{t} / \sup _{t}\left|\lambda_{t}\right|$ was chosen.

copula with estimated parameter $\hat{\theta}_{t}$ at time $t$ and let $C_{0}\left(u_{t}, v_{t}, \theta_{t}^{0}\right)$ be the true copula where $\theta_{t}^{0}$ denotes the true parameter at time $t$. Our hypothesis is

$$
H_{0}: C_{i}\left(u_{t}, v_{t}, \hat{\theta}_{t}\right)=C_{0}\left(u_{t}, v_{t}, \theta_{t}^{0}\right)
$$

As an estimator of $\theta_{t}$ we consider the smoothed paths $\hat{\theta}_{t \mid T}$ resulting from the importance sampler. Concerning the choice of the goodness-of-fit test many of the tests proposed in the papers cited above such as e.g. the bivariate $\chi^{2}$ test, are not suitable for time-varying copula models. A class of tests that is easily adaptable for the time-varying case is based on the fact that the conditional copula, i.e. the copula of $u$ given $v$ (or of $v$ given $u$ ), is uniformly distributed, which is an application of the Rosenblatt transformation. In our case this means

$$
z_{t}=C_{0}\left(u_{t} \mid v_{t}, \theta_{t}^{0}\right)=\frac{\partial C_{0}\left(u_{t}, v_{t}, \theta_{t}^{0}\right)}{\partial v_{t}} \sim U(0,1)
$$

Testing the copula specification therefore means testing whether $\hat{z}_{t}=C\left(\hat{u}_{t} \mid \hat{v}_{t}, \hat{\theta}_{t \mid T}\right)$ has a $U(0,1)$ distribution. For static copulas a closely related test has been considered in Breymann, Dias, and Embrechts (2003) and studied via Monte Carlo simulations in Dobric and Schmid (2007). For testing the uniformity of $\hat{z}_{t}$, various tests are available and we will consider the Kolmogorov-Smirnov (KS) test, the $\chi^{2}$ test, the Anderson-Darling (AD) test and the Jarque-Bera (JB) test for normality, for 
which we need to apply the transformation $\Phi^{-1}\left(\hat{z}_{t}\right)$. Let the empirical distribution function of $\hat{z}_{t}$ be

$$
\hat{\mathbb{F}}(x)=\frac{1}{T} \sum_{t=1}^{T} \mathbf{I}_{\left\{z_{t}<x\right\}}
$$

and let the CDF under the null hypothesis be $F(x)$. Then the KS test statistic is defined as

$$
T_{K S}=\sup _{x}|F(x)-\hat{\mathbb{F}}(x)|,
$$

for which critical values have been tabulated. In practice, the supremum is replaced by the maximum over the observations. For the $\chi^{2}$ test, consider splitting the domain $(0,1)$ in $k$ bins and let $c_{i}$ be the number of observations in bin $i$. Then the statistic of interest is

$$
T_{\chi^{2}}=\sum_{i=1}^{k} \frac{\left(E\left(c_{i}\right)-c_{i}\right)^{2}}{E\left(c_{i}\right)} \sim \chi^{2}(k),
$$

where the expectations of $c_{i}$ are taken under the null model. Next, let $S$ be sample skewness and $K$ be the sample kurtosis of $\Phi^{-1}\left(\hat{z}_{t}\right)$. Then the JB test is

$$
T_{J B}=\frac{T}{6}\left(S^{2}+\frac{(K-3)^{2}}{4}\right) \sim \chi^{2}(2) .
$$

Finally, the AD, which is a refinement of the KS test that is suitable to test deviations in the tails of the distribution, is given by

$$
T_{A D}=\sup _{x} \frac{\sqrt{T}|\hat{\mathbb{F}}(x)-F(x)|}{\sqrt{F(x)(1-F(x))}},
$$

for which again tabulated critical values are used.

\subsubsection{Forecasting}

A big advantage of specifying the underlying dependence process by an $\operatorname{AR}(1)$ structure is that it allows for easy forecasting. In contrast to the DCC model we can compute r-step ahead forecasts without making any approximations as outlined in Engle and Sheppard (2001). The techniques for forecasting AR(1) processes are standard and can be found in e.g. Hamilton (1994). With an estimate $\hat{\lambda}_{T}$, for which the smoothed estimate $\hat{\lambda}_{T \mid T}$ is suitable, the r-step ahead forecast of $\lambda$ is given by

$$
\hat{\lambda}_{T+r}=\mu+\beta^{r}\left(\hat{\lambda}_{T}-\mu\right)
$$


where $\mu=\alpha /(1-\beta)$. The mean squared r-period-ahead forecast error for $\lambda$ is

$$
\sigma_{T+r}^{2}=\nu^{2}\left(1-\beta^{2 r}\right) /\left(1-\beta^{2}\right)
$$

Unfortunately, only in the case of the Frank copula are we interested in forecasting $\lambda_{t}$ itself, but generally we want to forecast a nonlinear transformation thereof. In the case of the Clayton and Gumbel copulas we can use the following results. For $\lambda_{t} \mid \mathscr{F}_{t-1} \sim N\left(\mu_{t}, \sigma_{t}\right)$ the 1-step ahead forecast of $\theta_{t}=\exp \left(\lambda_{t}\right)$ is

$$
E\left(\theta_{t} \mid \mathscr{F}_{t-1}\right)=\exp \left(\mu_{t}+\frac{\sigma_{t}^{2}}{2}\right)
$$

For $\mu_{t}$, the conditional expectation of $\lambda_{t}$ given $\mathscr{F}_{t-1}$, we insert its linear forecast given in (6.34). From this it follows by straightforward calculations that the r-step ahead forecast of $\theta$ is

$$
\hat{\theta}_{T+r}=\exp \left(\hat{\lambda}_{T+r}+\frac{\sigma_{T+r}^{2}}{2}\right)
$$

The confidence bands for these forecasts can be used by using the corresponding quantiles of the log-normal distribution with parameters $\hat{\lambda}_{T+r}$ and $\sigma_{T+r}^{2}$.

Forecasting the correlation coefficient of the normal copula is not as straightforward due the nonlinearity of the inverse Fisher transform. We therefore use a Taylor approximation of $\Psi\left(\lambda_{t}\right)$ around $\mu_{t}$,

$$
\Psi\left(\lambda_{t}\right)=\Psi\left(\mu_{t}\right)+\Psi^{\prime}\left(\mu_{t}\right)\left(\lambda_{t}-\mu_{t}\right)+\frac{1}{2} \Psi^{\prime \prime}\left(\mu_{t}\right)\left(\lambda_{t}-\mu_{t}\right)^{2}
$$

Taking the conditional expectation we have

$$
\begin{aligned}
E\left[\Psi\left(\lambda_{t}\right) \mid \mathscr{F}_{t-1}\right] & =E\left\{\Psi\left(\mu_{t}\right)+\Psi^{\prime}\left(\mu_{t}\right)\left(\lambda_{t}-\mu_{t}\right)+\frac{1}{2} \Psi^{\prime \prime}\left(\mu_{t}\right)\left(\lambda_{t}-\mu_{t}\right)^{2} \mid \mathscr{F}_{t-1}\right\} \\
& =\Psi\left(\mu_{t}\right)+\Psi^{\prime}\left(\mu_{t}\right) E\left(\left(\lambda_{t}-\mu_{t}\right) \mid \mathscr{F}_{t-1}\right)+\frac{\Psi^{\prime \prime}\left(\mu_{t}\right)}{2} E\left(\left(\lambda_{t}-\mu_{t}\right)^{2} \mid \mathscr{F}_{t-1}\right) \\
& =\Psi\left(\mu_{t}\right)+\frac{\Psi^{\prime \prime}\left(\mu_{t}\right)}{2} \nu^{2} .
\end{aligned}
$$

Then the r-step ahead forecast is

$$
\hat{\theta}_{T+r}=\Psi\left(\hat{\lambda}_{T+r}\right)+\frac{-4\left(\exp \left(2 \hat{\lambda}_{T+r}\right)-1\right) \exp \left(2 \hat{\lambda}_{T+r}\right)}{\left(\exp \left(2 \hat{\lambda}_{T+r}\right)+1\right)^{3}} \sigma_{T+r}^{2} .
$$


Of course, higher order approximations could be used, but we did not find any substantial differences in our applications. Confidence bands are obtained by applying the inverse Fisher transform to the corresponding quantiles of the normal distribution.

\subsubsection{Tail properties of the Gaussian SCAR model}

Manner and Segers (2009) study the dependence in the tails of mixtures of Gaussian copulas. They show that when the random correlation parameter $\rho$ can get close to one with positive probability the tails of the mixture copula are much heavier than those of the static Gaussian copula. This situation applies to the Gaussian SCAR model. In particular, it is shown that the mixture copula has the property of near-independent tails as studied in Ledford and Tawn (1996), which means that for every $\varepsilon>0$ it holds that $u^{1+\varepsilon} \ll C(u, u) \ll u$ as $u \downarrow 0$, where $a(u) \ll b(u)$ means that $a(u)=o(b(u))$, that is $a(u) / b(u) \rightarrow 0$. This means that although $P[V<u \mid U<u] \rightarrow 0$ as $u \rightarrow 0$, the speed of convergence is extremely slow and at any practically relevant quantile the tail probability is substantially different from zero and hence at finite samples one gets the impression of asymptotically dependent tails. Consequently, one can expect the model to fit financial data well and in most situations the popular $t$-copula loses its advantage of being able to capture tail dependence. Economically, this property also has a nice interpretation. Just like GARCH or SV models imply fat tails in the univariate case, stochastic correlations create fat tails in the unconditional multivariate distribution. This might be seen as an extension of the "mixtures of distributions hypothesis" for stock prices introduced by Clark (1973).

\subsubsection{Possible extensions}

So far we have only considered stochastic copula models for bivariate copulas with a single dependence parameter. Here we discuss potential extension for bivariate models and some ideas that look promising for extending the model to larger dimensions.

\section{Extensions of the bivariate model}

Consider the conditional copula mixture density with time-varying mixing parameter

$$
c_{m i x}\left(u, v ; \theta_{1}, \theta_{2}, \kappa_{t}\right)=\kappa_{t} c_{1}\left(u, v ; \theta_{1}\right)+\left(1-\kappa_{t}\right) c_{2}\left(u, v ; \theta_{2}\right)
$$


where the mixing parameter $\kappa_{t}=\Psi\left(\lambda_{t}\right)$ with $\lambda_{t}$ following (6.3) and $\Psi(x)=1 /(1+\exp (x))$ to keep the mixing parameter in $(0,1)$ at all times. For example, $c_{1}$ and $c_{2}$ could be the Gaussian and the rotated Gumbel copulas and hence a decrease in $\kappa_{t}$ would cause an increase in lower tail dependence. Such a model could describe tranquil periods, when a Gaussian dependence structure is appropriate, and crisis periods with larger overall dependence and in particular greater dependence for losses.

The symmetrized Joe-Clayton copula introduced by Patton (2006b) is a flexible two-parameter copula that is parameterized in terms of $\lambda_{L}$ and $\lambda_{U}$, the coefficients of lower and upper tail dependence. A time-varying version of this model, as considered in Patton (2006b), allows for changing degrees of asymmetry, as well as a time-varying overall level of dependence. Again $\Psi(x)=1 /(1+\exp (x))$ could be chosen. For estimation the two component EIS procedure by Liesenfeld and Richard (2003) could be used. However, the estimation is likely to be computationally very burdensome. Furthermore, in Patton (2006b) the time-varying version of this model hardly has a better fit than a dynamic Gaussian copula, but three additional parameters need to be estimated.

An exogenous variable $x$ may be integrated into the models by replacing (6.3) by

$$
\lambda_{t}=\alpha+\beta \lambda_{t-1}+\delta x_{t}+\nu \varepsilon_{t} .
$$

The variable $x$ may be deterministic, such as a trend or a dummy representing a structural break, but it may also be an economic variable that is expected to explain correlation. A possibility would be to include trade volume, which could serve as a proxy for the impact of volatility on correlation.

\section{Multivariate modeling}

Extending the SCAR model to more than two dimensions is far from trivial. Archimedean copulas such as the Gumbel or Clayton copula have straightforward multivariate analogues, with the drawback that a single dependence parameter describes the complete dependence between all variables, which may be too restrictive for most applications. Similarly, for Gaussian copulas one could restrict all correlations to be equal leading to a model similar to the dynamic equicorrelation model by Engle and Kelly (2008).

Nevertheless, the recent advances in multivariate copula modeling using so called vine-copulas introduced by Bedford and Cooke (2002) and applied to financial data by e.g. Chollete et al. (2009) seem to offer a promising approach. We do not discuss the construction of vine copulas (also called pair copula constructions) here, but we note that the log-likelihood function decomposes nicely into a sum of the marginal log-likelihood and bivariate (conditional) copula log-likelihoods. We refer to Aas, 
Czado, Frigessi, and Bakken (2009) for a nice exposition of the details on pair copula constructions and their estimation. Thus the estimation of a rather complex model can be done in a number of relatively simple steps and the time-varying dependence processes can be estimated individually, but conditional on the estimated dependence process in earlier steps.

For Gaussian copulas an alternative to vine copulas could be to construct a time-varying correlation matrix $R_{t}$ by simply joining correlations obtained from bivariate Gaussian SCAR models. The obvious disadvantage of this approach is that $R_{t}$ may not be positive definite at all points in time, which is likely to be a serious problem for larger dimensions. This may be overcome by using shrinkage methods such as those proposed in Ledoit and Wolf (2004). However, optimal shrinkage rates and appropriate targets need to be developed. Moreover, it is not clear whether such an estimate for $R_{t}$ has good properties given the inefficient estimation and the bias due to shrinkage.

\subsection{Application}

In this section we present an empirical application of our model and compare it with competing models using two different data sets on stock indices, one measured at the daily frequency and during mostly tranquil market conditions, the second one at the weekly frequency and including a financial crisis at the end of the sample. The analysis is split up into estimation and in-sample validation, and out-of-sample comparison.

\subsubsection{Daily data: Dow Jones and NASDAQ}

The first data set we consider to illustrate our model and compare it with competing models are daily observations of the Dow Jones Industrial Average and the NASDAQ composite ranging from March 26, 1990 until March 23, 2000. The same data set has been considered in Engle (2002). Returns are calculated as the first difference of the natural logarithm multiplied by 100 .

\section{Estimation and in-sample validation}

In a first estimation step, a stochastic volatility (SV) model is fit to the demeaned returns. ${ }^{4}$ The SV model is estimated by EIS as described in Liesenfeld and Richard (2003). As a comparison we also estimate a standard GARCH(1,1) model. Parameter estimates and the values of the maximized loglikelihood function are given in Table 6.2, and the GARCH and smoothed SV volatilities can be found

\footnotetext{
${ }^{4} \mathrm{An} \mathrm{AR}(1)$ model for the conditional mean was also considered, but estimates for the volatility and dependence models were almost identical.
} 
Table 6.2: Estimates of GARCH and SV models: Dow Jones and Nasdaq

\begin{tabular}{ccc|ccc}
\hline \hline GARCH & DJ & NQ & SV & DJ & NQ \\
\hline$\alpha$ & 0.0060 & 0.0308 & $\alpha$ & -0.0112 & -0.0054 \\
& $(0.0044)$ & $(0.0173)$ & & $(0.0051)$ & $(0.0042)$ \\
$\gamma$ & 0.0480 & 0.1150 & $\beta$ & 0.9786 & 0.9733 \\
& $(0.0184)$ & $(0.0435)$ & & $(0.0076)$ & $(0.0078)$ \\
$\beta$ & 0.9450 & 0.8630 & $\nu$ & 0.1573 & 0.2072 \\
& $(0.0214)$ & $(0.0529)$ & & $(0.0254)$ & $(0.0266)$ \\
$\log \mathrm{l}$ & -3195.38 & -3693.89 & $\log l$ & -3137.37 & -3638.03 \\
\hline \hline
\end{tabular}

Table 6.3: Estimates of the stochastic copula model: Dow Jones and Nasdaq.

\begin{tabular}{lccc|ccc}
\hline \hline copula/logl & $\alpha$ & $\beta$ & $\nu$ & $\alpha$ & $\beta$ & $\nu$ \\
\hline Normal & 0.0302 & 0.9679 & 0.0824 & 0.0261 & 0.9720 & 0.0754 \\
902.169 & $(0.0099)$ & $(0.0103)$ & $(0.0147)$ & $(0.0089)$ & $(0.0093)$ & $(0.0135)$ \\
& & & & & & \\
rot. Gumbel & -0.0015 & 0.9795 & 0.1078 & -0.0013 & 0.9786 & 0.1111 \\
866.7934 & $(0.0023)$ & $(0.0084)$ & $(0.0236)$ & $(0.0024)$ & $(0.0085)$ & $(0.0235)$ \\
& & & & & & \\
Frank & 0.1527 & 0.9750 & 0.5457 & 0.1422 & 0.9768 & 0.5229 \\
790.9929 & $(0.0613)$ & $(0.0098)$ & $(0.1228)$ & $(0.0537)$ & $(0.0086)$ & $(0.1103)$ \\
& & & & & & \\
Clayton & 0.0136 & 0.9611 & 0.1549 & 0.0154 & 0.9636 & 0.1445 \\
752.0481 & $(0.0071)$ & $(0.0175)$ & $(0.0407)$ & $(0.0067)$ & $(0.0137)$ & $(0.0319)$ \\
\hline \hline
\end{tabular}

Note: Estimation results for the stochastic copula models with a Gaussian SV models as marginal distribution. Two-step estimation results in columns 2 to 4 and one-step estimation results in columns 5 to 7 .

in Figure 6.1. Not surprisingly, the SV model provides a better fit than the standard GARCH model due to its higher flexibility, and the GARCH model estimates a slightly higher degree of persistence. However, the estimated volatility series look very similar.

Using the estimated standard deviations, the data is transformed into $U(0,1)$ random variables using the probability integral transform, and the stochastic copula model is estimated. ${ }^{5}$ In order to assess the stability of our estimation procedure, we also estimate the model in one-step to obtain fully efficient estimates using the estimates of the two-step estimation as starting values. For the one-step estimator, standard errors are obtained by the inverse of the numerically evaluated Hessian. Standard errors for the two step estimates are obtained by evaluating (6.18) using numerical derivatives. Table

\footnotetext{
${ }^{5}$ We choose $N$, the number of simulated trajectories in the EIS sampler, equal to 500 in order to eliminate the Monte Carlo variation. A much smaller number such as 200 gives almost exactly the same estimates.
} 
Figure 6.1: Volatility estimates: Dow Jones and Nasdaq
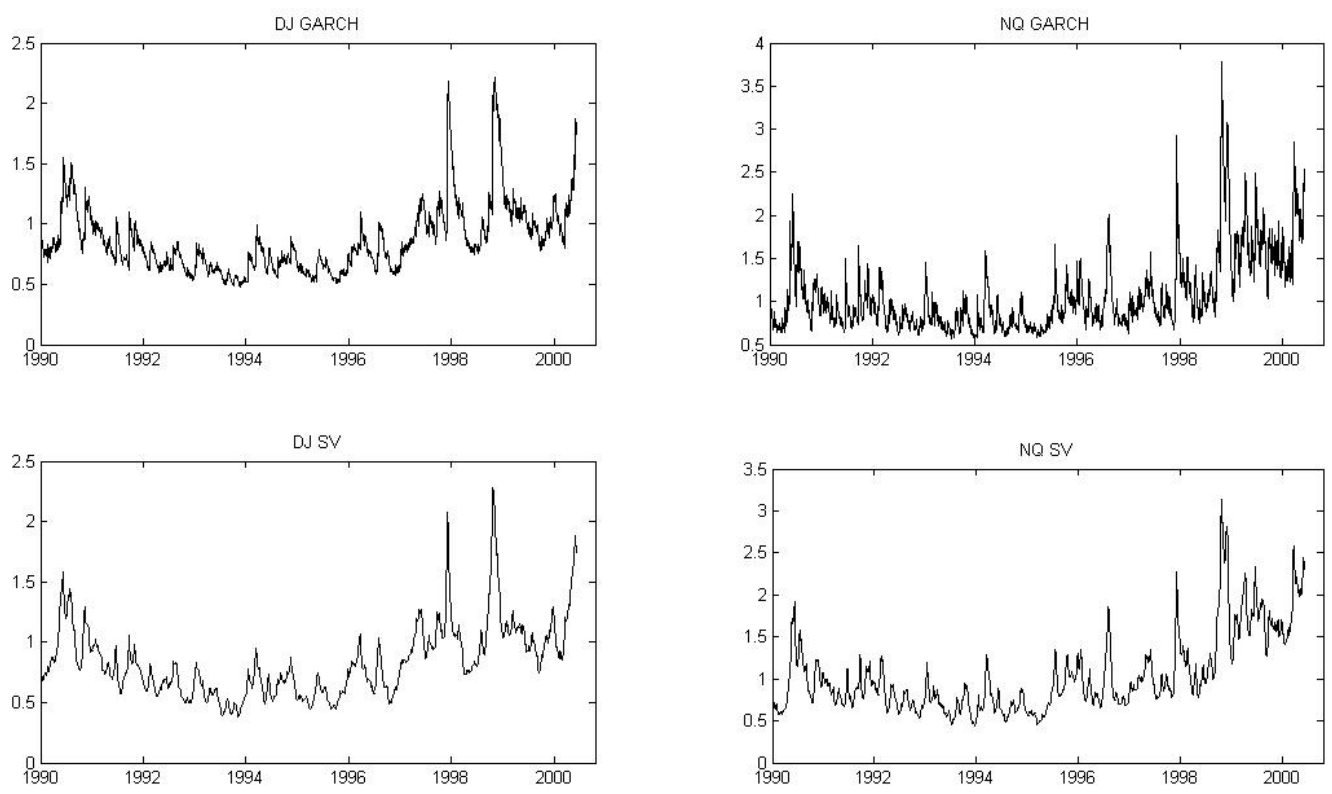

6.3 presents the results of the estimation. The first thing to observe is that for all models the dependence process is highly persistent, which is in line with findings in earlier studies. In terms of the value of log-likelihood, the Gaussian copula is the best fitting model, followed by the survival Gumbel copula. Comparing one-step and two-step estimates, one can see that they do not differ substantially. The standard errors for the two-step estimates are slightly higher, as expected. Only for the rotated Gumbel copula they are about equal, which could be attributed to numerical imprecisions. In Figure 6.2 , the smoothed estimate of the copula parameter is shown. It also includes the correlation path from the DCC and Patton models as a comparison. The path of the dependence parameters looks quite similar for all stochastic copula models, although the scale is different. The DCC correlation is a bit noisier, which is probably due to the fact that it is based on one-step ahead forecasts. Economically the decrease in dependence in 2000 was explained by Engle (2002) as the "sector rotation between new economy stock and and 'brick and mortar' stocks". Note that the Patton model fails to capture this drop in dependence. The outcomes of the Goodness-of-fit (GoF) tests are reported in Table 6.4. Note that the likelihood-ratio tests for constancy were also performed and led to a rejection of the null of constant dependence with p-values being essentially zero for all models. We also include the results for the Patton model for the Gaussian copula and the DCC model. Note that the DCC was estimated on the transformed variables $\Phi^{-1}(u)$ and $\Phi^{-1}(v)$, where $\Phi$ denotes the CDF of the standard 
Figure 6.2: Time path of the dependence parameters: Dow Jones and Nasdaq
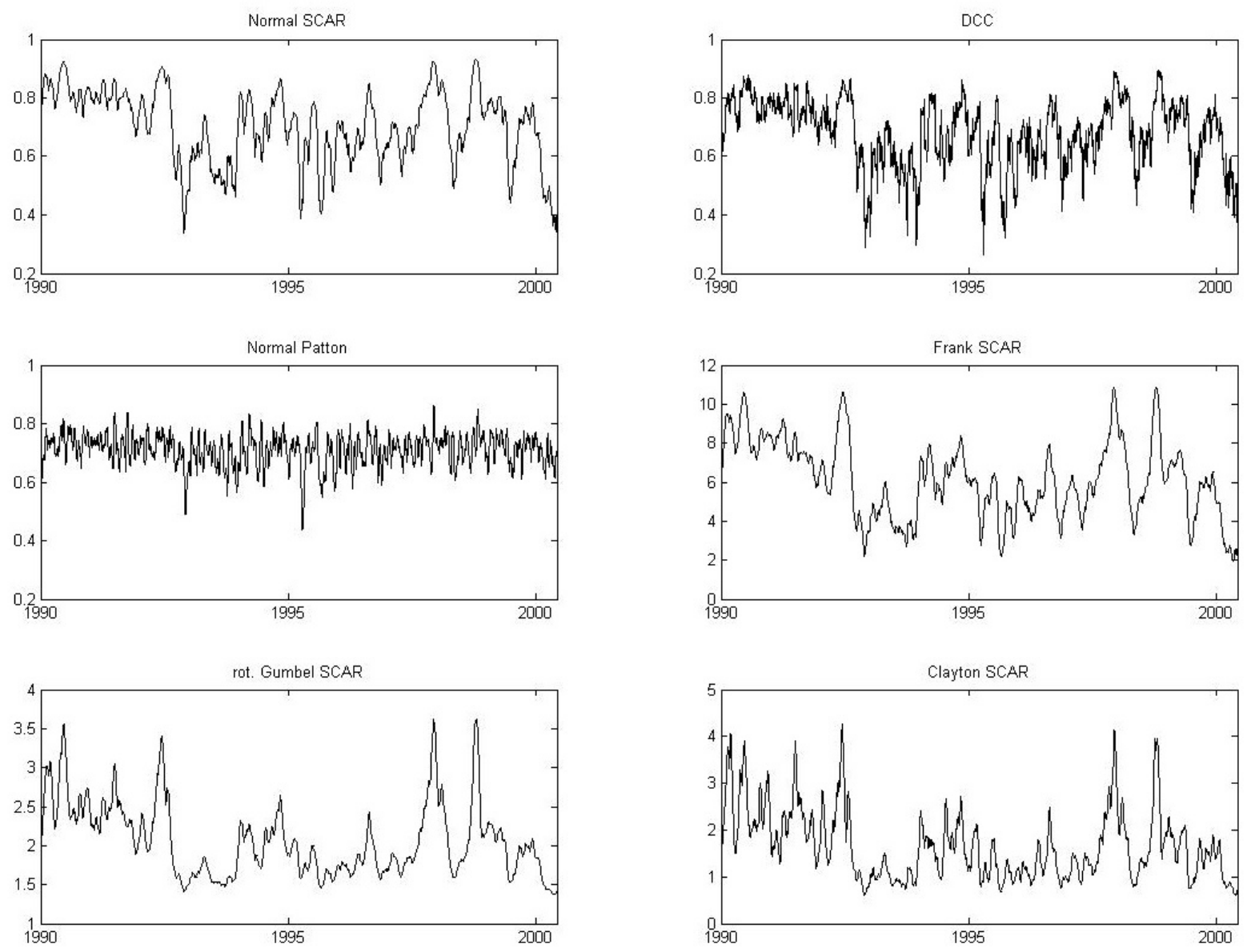

normal distribution. This was done to prevent differences in the copula fit due to different marginal distributions. The Gaussian SCAR model estimated in one step is the only model that passes all four tests. The Gaussian copula using the DCC and Patton models for time-varying correlation clearly have a worse in-sample fit. Next, it is surprising that the Gaussian copula outperforms the two asymmetric models for which losses have a higher degree of dependence than large returns, even though asymmetric models are preferred when considering a static copula model ${ }^{6}$. It seems that to some extent, this asymmetry is accounted for by the time-varying dependence parameter. Furthermore, the asymmetric models, in particular the Clayton copula, may simply underestimate the dependence for larger observations and this may outweigh the advantage of allowing for lower tail dependence. Also recall from Section 6.2.7 that the Gaussian SCAR model implies near independent tails and hence can capture dependencies in the extremes at finite samples.

\footnotetext{
${ }^{6}$ Estimation results for static copulas are available upon request.
} 
Table 6.4: Goodness-of-Fit tests: Dow Jones and Nasdaq.

\begin{tabular}{lcccccc}
\hline \hline Two step & Normal & rot. Gumbel & Frank & Clayton & DCC & Patton \\
\hline logl & 902.17 & 866.79 & 790.99 & 752.05 & 876.51 & 837.98 \\
KS & 0.2130 & 0.0046 & 0.0777 & 0.0001 & 0.1006 & 0.0392 \\
$\chi^{2}$ & 0.0012 & 0.0000 & 0.0001 & 0.0000 & 0.0001 & 0.0000 \\
JB & 0.0348 & 0.0000 & 0.0000 & 0.0000 & 0.0223 & 0.0014 \\
AD & 0.0303 & 0.0000 & 0.0000 & 0.0000 & 0.0155 & 0.0009 \\
\hline One step & & & & & & \\
KS & 0.2105 & 0.0043 & 0.0996 & 0.0001 & 0.1854 & 0.0404 \\
$\chi^{2}$ & 0.0527 & 0.0000 & 0.0000 & 0.0000 & 0.0010 & 0.0000 \\
JB & 0.0913 & 0.0000 & 0.0000 & 0.0000 & 0.0049 & 0.0010 \\
AD & 0.0584 & 0.0000 & 0.0000 & 0.0000 & 0.0039 & 0.0003 \\
\hline \hline
\end{tabular}

Note: P-values for the null hypothesis of correct specification of the copula function for the two-step (top panel) and one-step estimation (bottom panel) of the Dow Jones and Nasdaq data. KS, $\chi^{2}, \mathrm{JB}$ and $\mathrm{AD}$ are defined in equations (6.30) to (6.33).

\section{Out-of-sample comparison}

As a start we use the techniques described in Section 6.2.6 to obtain out-of-sample forecasts of the copula dependence process. We use the last observation of the smoothed dependence process as our initial observation and forecast over a horizon ranging from 1 to 250 trading days. As the dependence process is not observable, it needs to be estimated to check the performance of the forecasts. This is done by re-estimating the model using the 250 out-of-sample observations and computing the smoothed path of the dependence parameter. Figure 6.3 presents the forecasts together with $95 \%$ confidence bands and the smoothed path. It is noteworthy that the confidence band for the Normal, rotated Gumbel and Clayton models are asymmetric, thus taking the distributional assumptions on the dependence process into account. Although they are on different scales, their width is comparable when measured in terms of Kendall's tau. After about 100 days, the forecast distribution corresponds to the stationary distribution. Thus, the width of the forecast bands at long horizons corresponds to the large variation of the dependence paths in Figure 6.2. Also note that the 1-day ahead forecasts are surprisingly far off from the realization of the path. This is due to the fact that the complete sample has been re-estimated using additional data, which changed the estimates of the dependence process, in particular near the end of the original sample. This could be avoided comparing $r$-step ahead forecasts only with realizations of the process using $T+r$ observations, where $T$ is the size of the original sample. The forecasts seem to be reasonably precise considering the difficult task of forecasting unobserved dependence parameters and the realizations stay within the confidence bands 
Figure 6.3: Out-of-sample forecasts of the dependence parameter: Dow Jones and Nasdaq
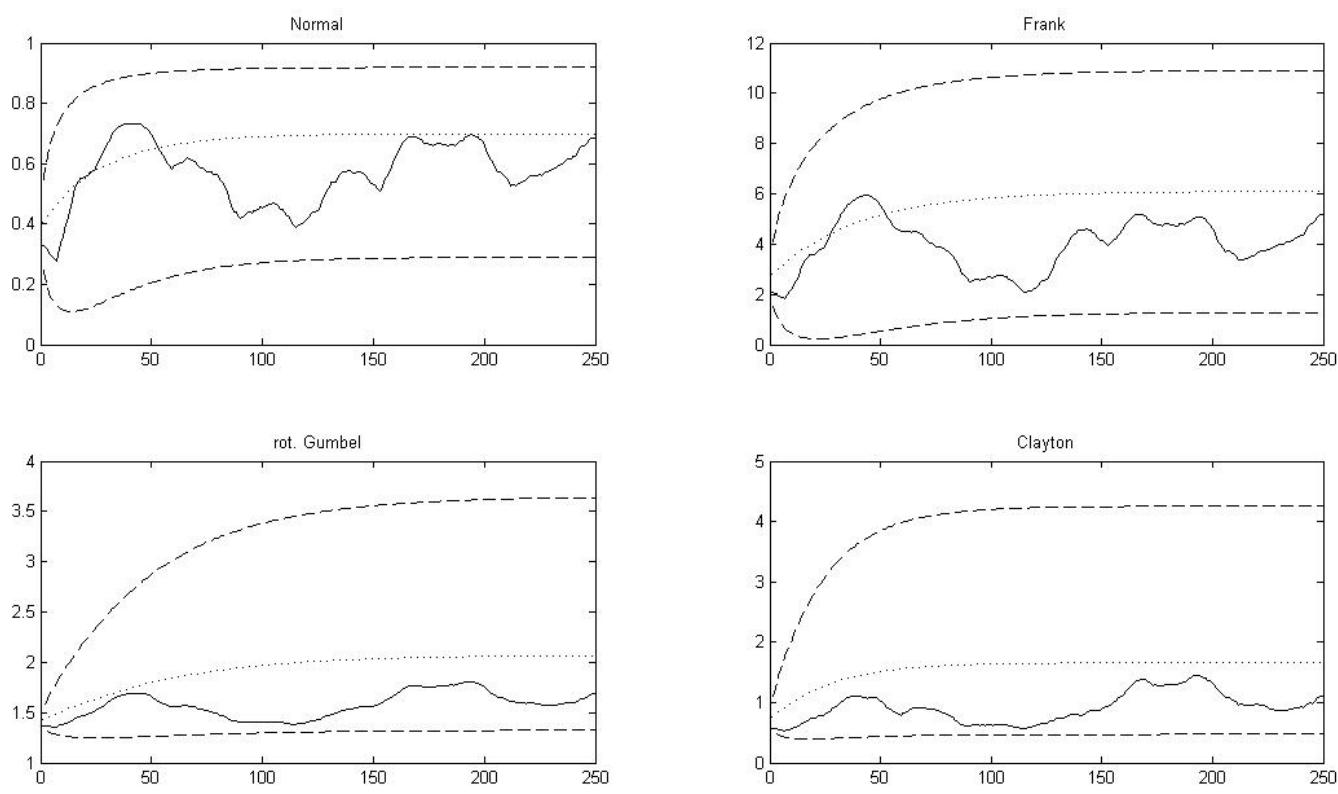

Note: The dotted lines denote the out-of-sample forecasts $\hat{\theta}_{T+r}$, the dashed lines are the $95 \%$ confidence intervals and the solid lines are the smoothed estimates $\hat{\theta}_{t \mid T}$.

at all times. So far our results suggest that stochastic copula model based on the Gaussian copula fits that data well in-sample and that the forecasts of the dependence path look reasonable when compared with the dependence implied by the model using the out-of-sample data. However, to show the usefulness of the forecasts of the copula parameter it is preferable to compare the forecasts to some measure that does not depend on a model. Given that the Gaussian copula had the best in-sample fit we exclusively focus on evaluating correlation and covariance forecasts in this section, which makes comparison to competing models and the use of established criteria possible. As in Pelletier (2006) we measure the closeness of an r-step forecast of the covariance matrix, which we denote $\hat{H}_{t+r}$, to the true covariance matrix by the following two criteria:

$$
\begin{aligned}
& R M S E_{r}=\left(\frac{1}{K^{2}} \sum_{i=1}^{K} \sum_{j=1}^{K}\left(\hat{H}_{i, j, t+r}-r_{i, t+r} r_{j, t+r}\right)^{2}\right)^{1 / 2}, \\
& M A D_{r}=\frac{1}{K^{2}} \sum_{i=1}^{K} \sum_{j=1}^{K}\left|\hat{H}_{i, j, t+r}-r_{i, t+r} r_{j, t+r}\right|
\end{aligned}
$$


Table 6.5: Out-of-sample fit: Dow Jones and Nasdaq

\begin{tabular}{llcccc}
\hline \hline & & CCC & DCC & SCAR-SV & SCAR-G \\
\hline MAD & $\mathrm{r}=1$ & 4.69 & 4.63 & $\mathbf{4 . 3 5}$ & 4.62 \\
& $\mathrm{r}=5$ & 4.76 & 4.72 & $\mathbf{4 . 3 2}$ & 4.70 \\
& $\mathrm{r}=10$ & 4.74 & 4.70 & $\mathbf{4 . 2 7}$ & 4.69 \\
& $\mathrm{r}=20$ & 4.27 & 4.25 & $\mathbf{3 . 8 5}$ & 4.23 \\
\hline RMSE & $\mathrm{r}=1$ & 10.29 & 10.32 & $\mathbf{1 0 . 2 5}$ & 10.31 \\
& $\mathrm{r}=5$ & 10.50 & 10.53 & $\mathbf{1 0 . 5 0}$ & 10.52 \\
& $\mathrm{r}=10$ & 10.60 & 10.63 & $\mathbf{1 0 . 5 9}$ & 10.62 \\
& $\mathrm{r}=20$ & $\mathbf{9 . 6 7}$ & 9.69 & 9.69 & 9.68 \\
\hline$\sigma_{M V P}$ & $\mathrm{r}=1$ & 1.45 & 1.43 & $\mathbf{1 . 4 3}$ & 1.44 \\
& $\mathrm{r}=5$ & $\mathbf{1 . 3 7}$ & 1.46 & 1.45 & 1.43 \\
& $\mathrm{r}=10$ & $\mathbf{1 . 3 6}$ & 1.49 & 1.50 & 1.47 \\
& $\mathrm{r}=20$ & $\mathbf{1 . 2 5}$ & 1.31 & 1.31 & 1.29 \\
\hline \hline
\end{tabular}

Note: This table presents the mean absolute deviation (MAD) and root mean square error (RMSE) between the forecasted covariance and the cross product of the out-of-sample data. The last panel shows the variance of the minimum variance portfolio (MVP) with weights given in (6.45).

where $K$ is the number of assets. The second criterion may be preferable because it is more robust to outliers. A third criterion on which we base our out-of-sample comparison could be linked to the economic value of the forecasts, namely the variance of the global minimum variance portfolio (MVP) constructed using the forecasted covariance matrix, see Fleming, Kirby, and Ostdiek (2001). The portfolio weights are given by

$$
w_{t+r}=\frac{\hat{H}_{t+r}^{-1} \iota}{\iota^{\prime} \hat{H}_{t+r}^{-1} \iota},
$$

where $\iota$ is a $(K \times 1)$ vector of ones.

We compare 1, 5, 10 and 20 step forecasts of the constant conditional correlation (CCC) GARCH, DCC-GARCH, SCAR-SV and the SCAR model with GARCH margins (SCAR-G). The SCAR model with GARCH margins was included to study the difference in forecasts that can be attributed to the correlation model. The parameters of the models are only estimated once using the in-sample period. We restrict the in-sample period to the last 1000 observations from the original sample to avoid effects of potentially unstable parameters, and since we need to run the computationally heavy importance sampler every time we want to forecast to evaluate the current volatility and correlation. For the out-of-sample period we consider the following 250 trading days. Table 6.5 reports the results of the analysis. The MAD and RMSE suggest that the SCAR-SV model has the best out-of-sample fit. Looking at $\sigma_{M V P}^{2}$ the simple CCC-GARCH model gives the best results, except for $r=1$, where 
Figure 6.4: Volatility estimates: CAC and DAX
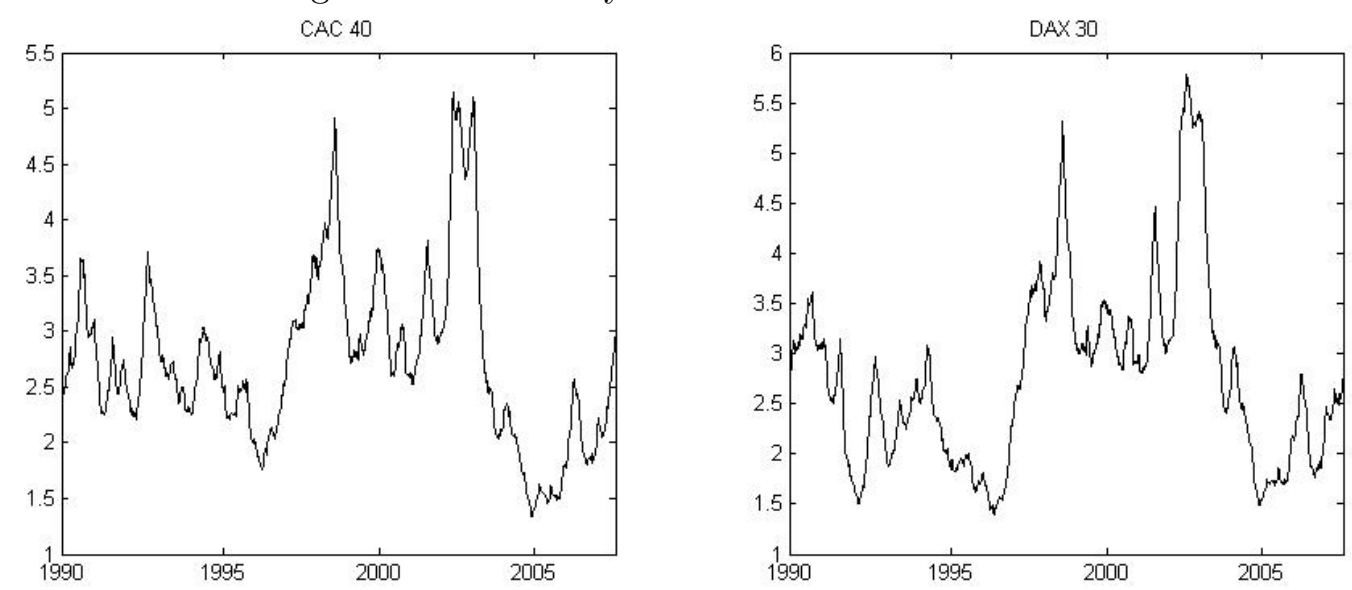

the SCAR-SV slightly outperform the DCC. This surprising finding is in line with the out-of-sample analysis of Pelletier (2006), who also found a good performance of a constant correlation model. This finding may be attributed to this specific data set. It is remarkable that comparing only the dynamic correlation models the SCAR does better than the DCC in all cases, except for $\sigma_{M V P}^{2}$ and $r=1$.

\subsubsection{Weekly data: CAC and DAX}

For the second application, we consider stock index returns observed at a weekly frequency. The series are the French CAC 40 and the German DAX 30 from January 1, 1990 until August 24, 2009 and thus also cover the recent financial crisis. The last 100 observations are put aside for the out-of-sample evaluation.

\section{Estimation and in-sample validation}

As above, a SV model is chosen for the margins and the SCAR model with different choices of copulas is considered for the dependence model. Only the two-step estimator is used as the estimates are quite close to those using one-step estimation. Since for two of the models the estimate for $\beta$ achieves the upper bound of 0.9999 the numerical derivatives could not be evaluated and hence standard errors of the estimates could not be obtained. Volatility estimates can be found in Figure 6.4. Note the high levels of volatility at the end of the 1990's and around the 9/11/2001 terrorist attacks. At the end of the in-sample period volatility seems to increase again, but the effect of the credit crisis mainly falls into the out-of-sample period. Estimates for the SCAR model and its competitors are reported in Table 6.6 and Figure 6.5. While in a static copula framework asymmetric models provides 
Table 6.6: Estimation and GoF for CAC and DAX returns.

\begin{tabular}{lcccccc}
\hline \hline & Normal & Gumbel & Frank & rot. Gumbel & DCC & Patton \\
\hline $\log \mathrm{l}$ & 575.20 & 531.15 & 532.03 & 543.44 & 565.11 & 498.80 \\
$\alpha$ & 0.0001 & 0.0284 & 0.0001 & 0.0219 & 0.0209 & 3.1171 \\
$\beta$ & 0.9999 & 0.9219 & 0.9999 & 0.9617 & 0.9792 & -1.9257 \\
$\nu$ & 0.0294 & 0.2749 & 0.2330 & 0.1764 & - & 1.0604 \\
\hline GoF & & & & & & \\
KS & 0.2052 & 0.0321 & 0.5434 & 0.1546 & 0.0326 & 0.0226 \\
$\chi^{2}$ & 0.3174 & 0.0692 & 0.5964 & 0.0345 & 0.1438 & 0.0000 \\
JB & 0.0044 & 0.0030 & 0.4691 & 0.0062 & 0.0010 & 0.0010 \\
AD & 0.0185 & 0.0000 & 0.6784 & 0.0006 & 0.0002 & 0.0000 \\
\hline \hline
\end{tabular}

Note: Two-step estimation results of the SCAR models and p-values for the null hypothesis of correct specification of the copula function using the tests in (6.30) to (6.33) for the weekly $\mathrm{CAC}$ and DAX returns.

a better fit, in the time-varying case the Gaussian copula is again clearly the best fitting model when looking at the log-likelihood. The GoF tests, on the other hand, suggest that the Frank copula is the appropriate model. Similar to the example above, the SCAR model fits slightly better than the DCC model and clearly outperforms the Gaussian conditional copula model by Patton (2006b). Note that the estimate of $\beta$ for the Patton model, which is not restricted to be smaller than one in absolute value, does not have the expected sign. However, different starting values lead to the same parameter estimates and this is in fact in line with Patton (2006b) who estimates a (large) negative persistence parameter for his time-varying Joe-Clayton copula. For the SCAR model the estimates for the persistence parameter differ quite significantly across different copulas. The symmetric models, namely the Gaussian and Frank copulas, show very high persistence, attaining the upper bound in the constrained optimization, so their dependence processes are likely to be integrated. Differences can also be seen from the time paths of the dependence parameters in Figure 6.5. This shows that choosing different copulas does not necessarily just result in similar shapes of the dependence process with differently scaled dependence parameters, but that an asymmetric model may in fact imply different dynamics over time. It is also encouraging that both the SCAR and the DCC model are able to capture movements of the dependence parameter that resemble trends or regime shifts without explicitly including such features in the model. The strong rise in stock market dependence is likely to be a consequence of the European integration process and the introduction of the Euro in 1999. 
Figure 6.5: Time path of the dependence parameters: CAC and DAX
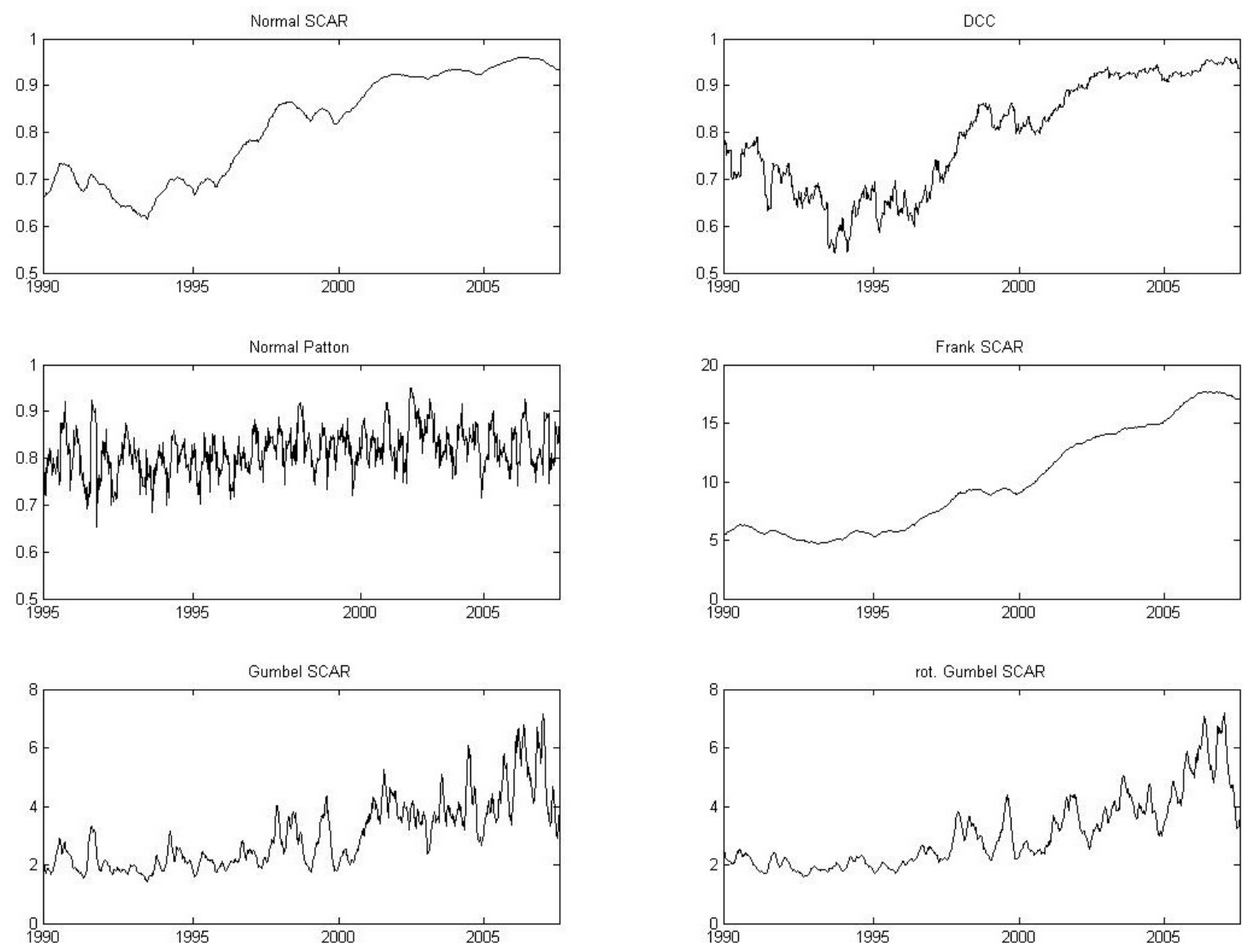

\section{Out-of-sample comparison}

The out-of-sample analysis is conducted in the same way as for the Dow Jones-Nasdaq data using the last 100 weeks of the sample. The results are reported in Table 6.7. Given that the credit crisis in 2008 is covered it is not surprising that now the CCC model does not beat the models that allow for correlation dynamics. The SCAR-SV model outperforms its competitors in the majority of cases. Comparing the DCC and SCAR with GARCH margins, the latter does better in terms of MAD and $\sigma_{M V P}^{2}$, indicating better forecasting performance of the SCAR model, in particular when measured in economic terms. It is remarkable that the model works well in a situation in which it is important to make good forecasts and when larger changes in dependence are likely to occur. 
Table 6.7: Out-of-sample fit: CAC and DAX

\begin{tabular}{lllccc}
\hline \hline & & CCC & DCC & SCAR-SV & SCAR-G \\
\hline MAD & $\mathrm{r}=1$ & 17.27 & 17.55 & $\mathbf{1 6 . 9 8}$ & 17.51 \\
& $\mathrm{r}=5$ & 17.68 & 18.00 & $\mathbf{1 7 . 4 9}$ & 17.94 \\
& $\mathrm{r}=10$ & 18.26 & 18.50 & $\mathbf{1 8 . 2 0}$ & 18.46 \\
& $\mathrm{r}=20$ & 18.13 & 18.21 & $\mathbf{1 7 . 8 0}$ & 18.19 \\
\hline RMSE & $\mathrm{r}=1$ & 33.28 & $\mathbf{3 3 . 1 8}$ & 33.21 & 33.19 \\
& $\mathrm{r}=5$ & 35.14 & 35.07 & $\mathbf{3 4 . 8 9}$ & 35.06 \\
& $\mathrm{r}=10$ & 36.13 & 36.00 & $\mathbf{3 5 . 9 7}$ & 36.01 \\
& $\mathrm{r}=20$ & 34.24 & 34.07 & $\mathbf{3 3 . 7 5}$ & 34.09 \\
\hline$\sigma_{M V P}^{2}$ & $\mathrm{r}=1$ & 19.07 & 19.01 & $\mathbf{1 8 . 6 7}$ & 18.91 \\
& $\mathrm{r}=5$ & 19.81 & 19.72 & $\mathbf{1 9 . 6 0}$ & 19.67 \\
& $\mathrm{r}=10$ & 20.79 & 20.88 & $\mathbf{2 0 . 6 6}$ & 20.84 \\
& $\mathrm{r}=20$ & $\mathbf{2 0 . 9 3}$ & 21.85 & 21.31 & 21.42 \\
\hline \hline
\end{tabular}

Note: This table presents the mean absolute deviation (MAD) and root mean square error (RMSE) between the forecasted covariance and the cross product of the out-of-sample data. The last panel shows the variance of the minimum variance portfolio (MVP) with weights given in (6.45).

\subsection{Conclusions}

We have proposed a stochastic copula model with a latent stochastic process driving the copula parameter. The model is discussed in various respects concerning specification, estimation, testing and forecasting. A simulation study compares the performance of the stochastic copula autoregressive (SCAR) model with that of the DCC model of Engle (2002) and of the Patton (2006b) model for alternative scenarios. In cases where all considered models are misspecified, the SCAR model clearly outperforms its competitors. In an empirical application we considered two pairs of stock index series, one on a daily, the other on a weekly frequency. In most cases, the SCAR model based on the Gaussian copula fits the data well and again outperforms the DCC and Patton models. The out-of-sample analysis shows good performance of the model compared the DCC and CCC GARCH models. In particular for the data including the credit crisis the model performs very well compared to its competitors.

We have discussed a number of possible extensions of the model. In particular the modeling or more than two assets appears to be an interesting, but very challenging, venue for future research. Furthermore, an interesting question is whether there are specific applications where asymmetric copulas outperform the Gaussian ones and hence the flexible and non-linear dependence structures such copulas capture can be exploited. 



\section{Tails of Gaussian and $t$-copulas with random correlations $^{1}$}

\subsection{Introduction}

It is a stylized fact that financial data such as stock or exchange rate returns exhibit positive tail dependence, and for that reason student $t$-copulas with low degrees of freedom often fit such data well. Furthermore, in several studies such as Erb et al. (1994), Longin and Solnik (1995) and Engle (2002) it is shown that correlations between financial time series are varying over time. Consequently, recent studies have modeled the dependence between financial variables such as stock or exchange rate returns using copula functions whose parameters are allowed to vary over time, see e.g. Patton (2006b), Hafner and Reznikova (2008) and Hafner and Manner (2008).

Tail dependence has been studied quite thoroughly in the literature on extreme value theory by, among many others, Ledford and Tawn (1996) and Heffernan (2000). For an overview, a comparison of competing techniques to estimate tail dependence, and a discussion of practical issues see Coles, Heffernan, and Tawn (1999) and Frahm, Junker, and Schmidt (2005).

In this chapter we focus on the specific case of tails of Gaussian and $t$-copulas whose correlation coefficient is itself a random variable. The general case of tails of elliptical distributions have been studied in the literature by Abdous, Fougères, and Ghoudi (2005), Asimit and Jones (2007) and Hashorva $(2005,2008,2009)$. However, our situation has not been covered by these studies. In fact, the copula one obtains when mixing over the correlation of elliptical copulas is not itself an elliptical copula as can easily be seen by looking at the contours of the mixture of bivariate normal distributions with correlations equal to -0.99 and 0.99 .

As a model for random correlations we focus on the following specification that has been introduced by $\mathrm{Yu}$ and Meyer (2006) in the context of multivariate stochastic volatility models and by

\footnotetext{
${ }^{1}$ This chapter is based on Manner and Segers (2009).
} 
Figure 7.1: Penultimate tail dependence of $t$-copulas
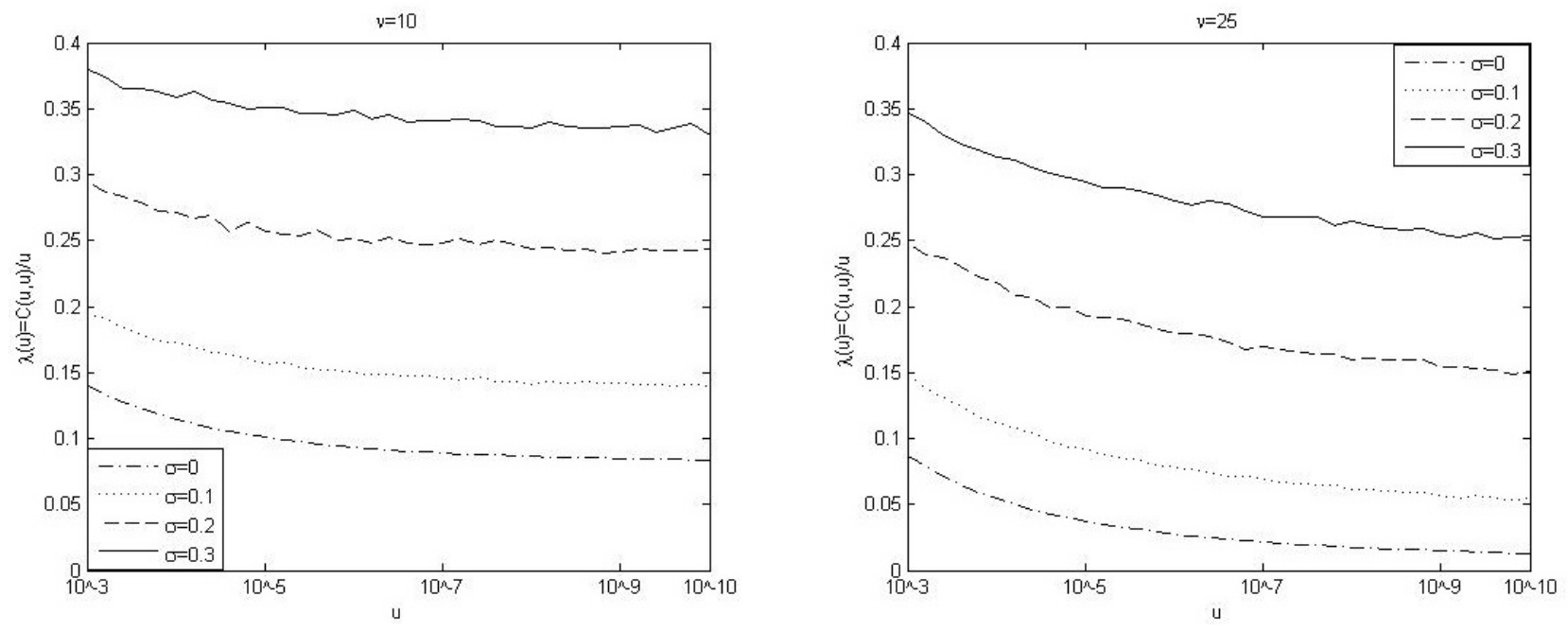

Hafner and Manner (2008) for time-varying copulas. Time-varying correlation $\rho_{t}$ is described by

$$
\begin{aligned}
& \gamma_{t}=\alpha+\beta \gamma_{t-1}+\sigma \varepsilon_{t} \\
& \rho_{t}=\left(\exp \left(2 \gamma_{t}\right)-1\right) /\left(\exp \left(2 \gamma_{t}\right)+1\right)
\end{aligned}
$$

where $\varepsilon_{t} \stackrel{\text { i.i.d. }}{\sim} N(0,1),|\beta|<1$, and the inverse Fisher transform is chosen to keep $\rho_{t}$ in $(-1,1)$ at all times. This specification is intuitively reasonable, analytically tractable and has been found to provide an excellent fit to financial data when used with a Gaussian copula. Nevertheless, all our results apply to more general specifications for random correlations and the model (7.1) merely serves as a plausible example.

Dependence in the tails is measured by the coefficient of penultimate tail dependence

$$
\lambda(u)=\frac{C(u, u)}{u}=\mathrm{P}(U<u \mid V<u)
$$

for copula $C$ and $0<u \leqslant 1$. If $\lambda(u) \rightarrow \lambda$ as $u \rightarrow 0$, then the limit $\lambda$ is the coefficient of tail dependence. We argue that $\lambda(u)$ is more relevant for practical purposes than $\lambda$, since for practical quantiles $u$ it is found to be much larger than in the limit. This phenomenon is depicted in Figure 7.1 where we plot $\lambda(u)$ as a function of $u$ as we look very far into the tail for $t$-copulas with correlations generated by (7.1) with unconditional correlation equal to 0.5 for all cases. This figure nicely demonstrates the main findings of this chapter. First, the penultimate tail dependence is significantly larger than its limit and the speed of convergence is extremely slow. Second, allowing for random correlation greatly 
increases both $\lambda(u)$ and $\lambda$. Similar effects can be observed when mixing over Gaussian copulas, but with $\lambda(u)$ converging to 0 at a very slow rate and being much larger beyond all practically relevant quantiles.

For practical purposes our findings imply that for assessing risks in the tails of the distribution financial variables it appears to be important to look at $\lambda(u)$ instead of its limit $\lambda$ and that it is crucial to take time-varying correlation into account when modeling the joint distribution of financial time series.

The rest of the chapter is structured as follows. In the next section the coefficient of (penultimate) tail dependence for elliptical copulas with random correlation parameter will be studied. In Section 7.3 we look at penultimate tail dependence and its rate of convergence as $u \rightarrow 0$ for Student and Gaussian copulas. Section 7.4 discusses the consequences of our results for modeling through a Monte Carlo study and an illustration on various financial time series. Finally, Section 7.5 concludes.

\section{2 (Penultimate) Tail dependence coefficients of mixture copulas}

Let $(X, Y)$ be a standardized bivariate elliptical random vector, i.e.

$$
(X, Y) \stackrel{d}{=}\left(S_{1}, \rho S_{1}+\left(1-\rho^{2}\right)^{1 / 2} S_{2}\right)
$$

where $-1 \leqslant \rho \leqslant 1$ and where

$$
\left(S_{1}, S_{2}\right) \stackrel{d}{=}(R \cos \Theta, R \sin \Theta)
$$

with $R$ and $\Theta$ independent random variables with $R>0$ and $\Theta$ uniformly distributed on $(-\pi, \pi)$. Consider the distribution of the radius $R$ as fixed. Let $C_{\rho}$ be the copula of $(X, Y)$ seen as parameterized by the correlation parameter $\rho$, that is,

$$
C_{\rho}(u, v)=\mathrm{P}(U \leqslant u, V \leqslant v), \quad(u, v) \in[0,1]^{2},
$$

with $U=F(X)$ and $V=F(Y)$, where $F$ is the common marginal distribution function of $X$ and $Y$.

Recall that the penultimate coefficient of tail dependence is defined by

$$
\lambda_{\rho}(u)=\frac{C_{\rho}(u, u)}{u}=\mathrm{P}(U<u \mid V<u)
$$

for $0<u \leqslant 1$. If $\lambda_{\rho}(u) \rightarrow \lambda_{\rho}$ as $u \rightarrow 0$, then the limit $\lambda_{\rho}$ is the coefficient of tail dependence. 
Proposition 7.1. For every $u \in(0,1 / 2]$, the function $\rho \mapsto \lambda_{\rho}(u)$ is convex in $\rho \in[0,1]$.

Graphs of $\lambda_{\rho}(u)$ for fixed $u$ suggest that the restriction to $\rho \in[0,1]$ cannot be avoided. Still, under some mild conditions on the distribution of $R$, the convexity is actually true on $\rho \in[-1,1]$ for all sufficiently small $u>0$. Let $F_{R}$ be the distribution function of $R$.

Proposition 7.2. Suppose that $F_{R}$ has unbounded support and is absolutely continuous with density $f_{R}$. If

$$
\liminf _{r \rightarrow \infty} \frac{r f_{R}(r)}{F_{R}(r)}>1
$$

then there exists $u_{0} \in(0,1 / 2)$ such that the function $\rho \mapsto \lambda_{\rho}(u)$ is convex in $\rho \in[-1,1]$ for every $u \in\left(0, u_{0}\right)$.

For the bivariate $t$-distribution with $\nu$ degrees of freedom (including the bivariate Gaussian with $\nu=\infty)$, we have

$$
\lim _{r \rightarrow \infty} \frac{r f_{R}(r)}{F_{R}(r)}=\nu
$$

Hence, condition (7.2) is satisfied if $\nu>1$. More generally, condition (7.2) is verified as soon as the radial density function $f_{R}$ is regularly varying at infinity of index $-\alpha-1$ for some $\alpha>1$, in which case the distribution of $R$ is in the max-domain of attraction of the Fréchet $(\alpha)$ distribution.

The relevance of the two propositions is the following. If the copula $C$ is a $\rho$-mixture of the copulas $C_{\rho}$, that is, if there exists a probability measure $\mu$ on $[-1,1]$ such that

$$
C(u, v)=\int_{-1}^{1} C_{\rho}(u, v) \mu(\mathrm{d} \rho)
$$

then the penultimate coefficient of tail dependence of $C$ is given by

$$
\begin{aligned}
\lambda(u) & =\frac{C(u, u)}{u} \\
& =\int_{-1}^{1} \frac{C_{\rho}(u, u)}{u} \mu(\mathrm{d} \rho)=\int_{-1}^{1} \lambda_{\rho}(u) \mu(\mathrm{d} \rho)
\end{aligned}
$$

for $u \in(0,1]$. Let

$$
\bar{\rho}=\int_{-1}^{1} \rho \mu(\mathrm{d} \rho)
$$

denote the average correlation parameter. If the function $\rho \mapsto \lambda_{\rho}(u)$ is convex, then by Jensen's inequality,

$$
\lambda(u) \geqslant \lambda_{\bar{\rho}}(u), \quad u \in(0,1]
$$


Figure 7.2: Tail dependence of static and dynamic $t$-copulas
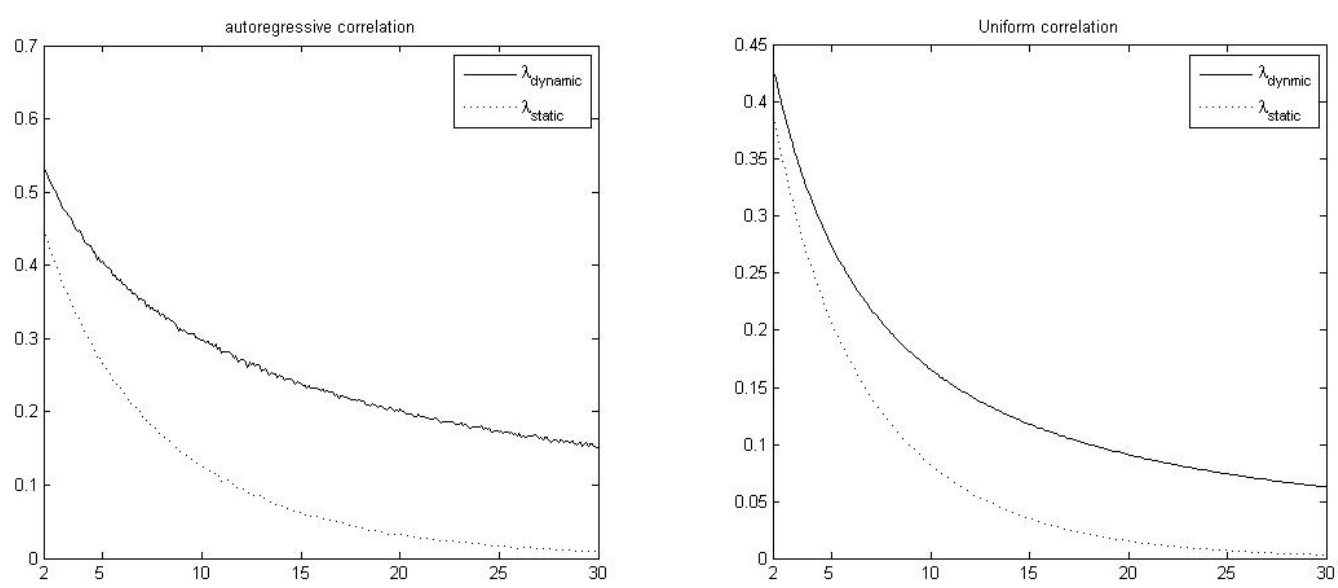

whatever the mixing distribution $\mu$.

The copula-mixture model (7.3) arises for instance in case of a time series $\left(X_{t}, Y_{t}\right)$ when the conditional distribution of $\left(X_{t}, Y_{t}\right)$ given the information available up to time $t-1$ is elliptical with correlation parameter $\rho_{t}$. The unconditional distribution of $\left(X_{t}, Y_{t}\right)$ is then of the form in (7.3), with $\mu$ the unconditional distribution of the (latent) random correlation parameter $\rho_{t}$. The interpretation of (7.5) then is that by allowing for time-varying correlation parameters, tail dependence may be larger than what one would at first expect.

For $\lambda(u)$ this phenomenon can be seen in Figure 7.1, whereas Figure 7.2 illustrates it for the coefficient of tail dependence $\lambda$. We consider Student $t$-copulas with stochastic and static correlations such that both have an unconditional correlation coefficient of 0.5 . The degrees-of-freedom parameter varies from 2 to 30. For the left graph the dynamic correlation is generated by (7.1), whereas for the right graph $\rho_{t} \sim U(0,1)$. The coefficient of tail dependence of the $t$-copula with $\nu>0$ degrees of freedom and correlation parameter $\rho \in(-1,1)$ is given by

$$
\lambda(\nu, \rho)=2 t_{\nu+1}(-\sqrt{\nu+1} \sqrt{1-\rho} / \sqrt{1+\rho})
$$

where $t_{\mu}$ denotes the cumulative distribution function of the $t$-distribution with $\mu>0$ degrees of freedom; see Demarta and McNeil (2005). Clearly the dynamic models lead to a significantly higher tail dependence than static models. In practice this in turn implies that falsely assuming a static $t$-copula when the data has in fact been generated by a time-varying $t$-copula will result in biased estimates of either the degrees of freedom parameter or the coefficient of tail dependence. Which of 
the two will be biased depends on the estimator applied and cannot be predicted in general. We will study this effect below both by simulations and real data examples.

\subsection{Penultimate tail dependence}

So far we have shown that mixtures over elliptical copulas have more tail dependence than static ones with the same overall dependence. In this section we look at the penultimate coefficient of tail dependence $\lambda(u)$ for Gaussian and Student $t$-copulas and the rate of convergence to its limit. This measure is more suited to assess the risks of joint extremes for practical purposes than the limiting coefficient of tail dependence, which is in general smaller.

Section 7.3.1 studies $\lambda(u)$ for bivariate $t$-copulas both with static and random correlations, whereas Section 7.3.2 looks at the tails of Gaussian copulas with random correlations

\subsubsection{Penultimate tail dependence of mixtures of bivariate $t$-copulas}

Let $\nu>0$ and $-1<\rho<1$. Let $Z_{1}, Z_{2}, S$ be independent random variables such that $Z_{i}$ is standard normal and $\nu S^{2}$ is chi-squared with $\nu$ degrees of freedom. Put

$$
X=S^{-1} Z_{1}, \quad Y=S^{-1}\left(\rho Z_{1}+\sqrt{1-\rho^{2}} Z_{2}\right)
$$

The distribution of the random vector $(X, Y)$ is bivariate $t$ with $\nu$ degrees of freedom and correlation parameter $\rho$. Its copula, $C$, is the bivariate $t$-copula with parameters $\nu$ and $\rho$.

Now again suppose that $\rho$ is itself a random variable taking values in $(-1,1)$, independent of $Z_{1}, Z_{2}, S$. The unconditional distribution (copula) of $(X, Y)$ is then a mixture of bivariate $t$ distributions (copulas) with fixed degrees of freedom parameter $\nu$.

We investigate the limit and the rate of convergence in

$$
\lambda(u)=\frac{C(u, u)}{u} \rightarrow \lambda, \quad u \downarrow 0 .
$$

The limit $\lambda$ is the coefficient of upper/lower tail dependence, whose value is given in (7.6), see Demarta and McNeil (2005). So our focus is on the rate of convergence to the limit. This is relevant for the penultimate behavior of joint extremes, that is, at high but finite thresholds. For real $x$, let $x_{+}=\max (x, 0)$ be its positive part. 
Proposition 7.3. For random or non-random $\rho$, we have

$$
\lambda(u)=\frac{C(u, u)}{u}=\lambda+\gamma u^{2 / \nu}+o\left(u^{2 / \nu}\right), \quad u \downarrow 0,
$$

where

$$
\lambda=\frac{E\left[\min \left(Z_{1}, \rho Z_{1}+\sqrt{1-\rho^{2}} Z_{2}\right)_{+}^{\nu}\right]}{E\left[Z_{+}^{\nu}\right]}
$$

and $\gamma$ is a certain positive constant depending on $\nu$ and the distribution of $\rho$, see (7.18) below.

\section{Remarks.}

1. For nonrandom $\rho \in(-1,1)$, the coefficient of tail dependence is equal to the value reported in Demarta and McNeil (2005) that can be found in equation (7.6). For general, random $\rho$, we find

$$
\lambda=\mathrm{E}[\lambda(\nu, \rho)]
$$

the expectation being with respect to $\rho$.

2. The rate of convergence of $C(u, u) / u$ to its limit $\lambda$ is of the order $O\left(u^{2 / \nu}\right)$. For large $\nu$, this rate is slow. Since moreover $\gamma>0$, the penultimate coefficient of tail dependence $\lambda(u)=C(u, u) / u$ may therefore be (much) larger than its limit, $\lambda$. As a result, at finite thresholds, the tail may look much heavier than it is in the limit. This in turn may cause estimators of $\nu$ to be negatively biased.

3. For $\nu \rightarrow \infty$ we recover the Gaussian distribution, for which the speed of convergence is known to be of logarithmic order only.

\subsubsection{Gaussian copulas with random correlation: Near asymptotic dependence}

Whereas the last section dealt with the Student $t$-copula, which includes the Gaussian copula when $\nu \rightarrow \infty$, in this section we exclusively focus on the Gaussian copula. Furthermore, the correlation coefficient is now also assumed to take on large values with positive probability, but besides that can be of any type. The stochastic correlation process in (7.1) is an example we focus on due to its simple and intuitive structure and because such a model was shown to fit financial data extremely well in Yu and Meyer (2006) and Hafner and Manner (2008). Thus, although the results below are not exclusive to the dynamic correlation model in (7.1) they provide an explanation for its good fit. 
Let $\left(X_{1}, X_{2}\right)$ be a random pair, let $R$ be a random variable taking values in the interval $(-1,1)$, and assume that conditionally on $R=\rho$, the distribution of $\left(X_{1}, X_{2}\right)$ is bivariate normal with mean zero, unit variance, and correlation $\rho$. The marginal distributions of $X_{1}$ and $X_{2}$ are then both standard normal and the copula of $\left(X_{1}, X_{2}\right)$ is

$$
C(u, v)=P\left[X_{1} \leqslant \Phi^{-1}(u), X_{2} \leqslant \Phi^{-1}(v)\right]=\int_{-1}^{1} C_{\rho}(u, v) d P[R \leqslant \rho]=E\left[C_{R}(u, v)\right]
$$

for $u, v \in[0,1]$; here $\Phi^{-1}$ denotes the inverse of the standard normal distribution function, $\Phi$, and $C_{\rho}$ is the bivariate Gaussian copula with correlation $\rho$. Note that we assume that $-1<R<1$ almost surely.

The objective here is to study the tail dependence properties of $\left(X_{1}, X_{2}\right)$. Since $\left(X_{1}, X_{2}\right)$ and $\left(-X_{1},-X_{2}\right)$ are equal in law, it suffices to consider the lower tail. Our primary objective then is to find the asymptotic behavior of $C(u, u)$ as $u \downarrow 0$.

By the assumption that $R<1$ almost surely, it is immediately clear that

$$
\lim _{u \downarrow 0} u^{-1} C(u, u)=\lim _{u \downarrow 0} \int_{-1}^{1} u^{-1} C_{\rho}(u, u) d P[R \leqslant \rho]=0 .
$$

Indeed, for each $\rho<1$ we have $u^{-1} C_{\rho}(u, u) \rightarrow 0$ as $u \downarrow 0$, a limit relation which can be integrated over $\rho \in(-1,1)$ in view of the dominated convergence theorem and the fact that $0 \leqslant u^{-1} C_{\rho}(u, u) \leqslant 1$ for all $u$ and $\rho$.

So just like Gaussian copulas themselves, mixtures of Gaussian copulas have asymptotically independent upper and lower tails, which is not surprising. However, the statement that the common value of $\lim _{x \rightarrow \infty} P\left[X_{1} \leqslant-x \mid X_{2} \leqslant-x\right]=\lim _{u \downarrow 0} u^{-1} C(u, u)$ is equal to zero needs some qualification: if the speed with which $u^{-1} C(u, u)$ converges to zero is slow, then for practically relevant values of $u$ the quantity $u^{-1} C(u, u)$ may be well different from zero. This is exactly what is happening here. Let $a(u) \ll b(u)$ be taken to signify $a(u)=o(b(u))$, that is, $a(u) / b(u) \rightarrow 0$.

Proposition 7.4. Let $C$ be as in (7.8). If $P(R>\rho)>0$ for all $\rho<1$, then for every $\varepsilon>0$,

$$
u^{1+\varepsilon} \ll C(u, u) \ll u, \quad u \downarrow 0 .
$$

Thus, for the unconditional copula in (7.8) the convergence of the conditional tail probability $P[U<u \mid V<u]$ as $u \downarrow 0$ is slower than $u^{\varepsilon}$ for every value of $\varepsilon>0$. To put this finding into 
perspective recall from Ledford and Tawn (1996) that the behavior in the tails is given by

$$
P[V<u \mid U<u]=\frac{C(u, u)}{u}=\mathscr{L}(1 / u) u^{1-\frac{1}{\eta}},
$$

where $\mathscr{L}$ is a slowly varying function, meaning $\mathscr{L}(t x) / \mathscr{L}(x) \rightarrow 1$ as $x \rightarrow \infty$ for all $t>0$, and the parameter $\eta$ is (also) called the coefficient of tail dependence. Ledford and Tawn (1996) speak of asymptotic dependence when $\eta=1$ and $\mathscr{L}(1 / u) \nrightarrow 0$ as $u \rightarrow 0$, of positive association for $1 / 2<\eta<1$, and of near independence when $\eta=1 / 2$ and $\mathscr{L}(1 / u) \geqslant 1$. In our case, however, we have the situation that $\eta=1$ and $\mathscr{L}(1 / u) \rightarrow 0$ as $u \rightarrow 0$, which, to our knowledge, has not been encountered in the literature until now. We shall denote this behavior by near asymptotic dependence to refer to the fact that even though the tails are not dependent in the limit we are extremely close to having tail dependence.

Changing variables $u=\bar{\Phi}(x)$ and exploiting the symmetry of the normal distribution allows us to write

$$
\begin{aligned}
C(\bar{\Phi}(x), \bar{\Phi}(x)) & =P\left[X_{1}>x, X_{2}>x\right] \\
& =P\left[X>x, R X+\sqrt{1-R^{2}} Y>x\right] \\
& =P\left[R X+\sqrt{1-R^{2}} Y>x \mid X>x\right] P[X>x],
\end{aligned}
$$

where $X$ and $Y$ are independent standard normal random variables, independent of $R$. In order to get more precise statements than the one in the Proposition 7.4, we need to analyse the first probability on the right-hand side of the previous display. More specific assumptions are required on the behavior of the distribution of $R$ near 1 , or equivalently of the one of $(1-R)^{-1}$ near infinity. Put

$$
G(t)=P\left[\frac{1}{1-R}>t\right]=P\left[R>1-\frac{1}{t}\right], \quad t>0
$$

Proposition 7.5. Let $X, Y, R$ be independent random variables, $X$ and $Y$ being standard normal and $-1 \leqslant R<1$. If the function $G$ in (7.11) is regularly varying at infinity with index $-\alpha<0$, then

$$
\begin{aligned}
\lim _{x \rightarrow \infty} \frac{1}{P\left[R>1-x^{-2}\right]} P\left[R X+\sqrt{1-R^{2}} Y>x \mid X\right. & >x] \\
& =E\left[\left(Z+Y^{2}+Y \sqrt{2 Z+Y^{2}}\right)^{\alpha}\right]=c(\alpha)
\end{aligned}
$$


Figure 7.3: $\lambda(u)$ and $\eta$ for the stochastic Gaussian copula
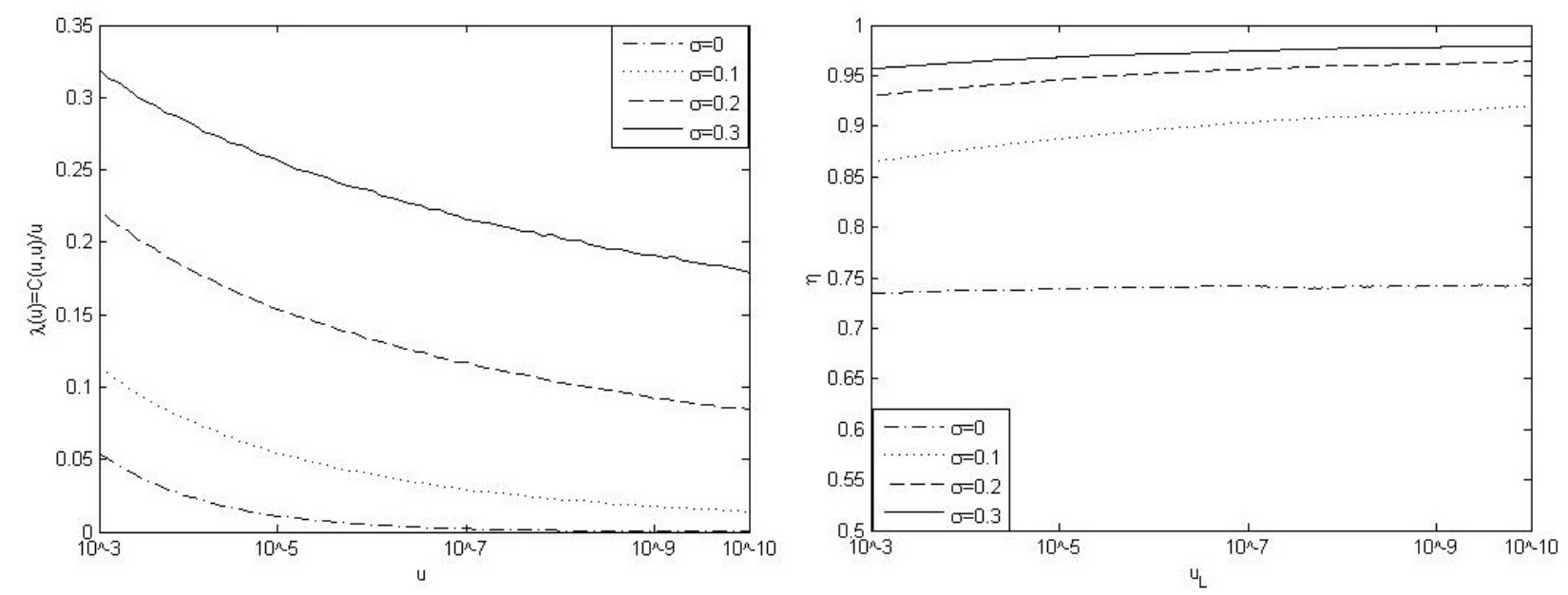

where $Z$ is standard exponential, independent of $Y$. In particular,

$$
C(u, u) \sim c(\alpha) u G(2 \log (1 / u)), \quad u \downarrow 0 .
$$

Addendum 7.1. The argument in the expectation on the right-hand side of (7.12) can also be written as

$$
Z+Y^{2}+Y \sqrt{2 Z+Y^{2}}=\frac{2 Z^{2}}{\left(\sqrt{2 Z+Y^{2}}-Y\right)^{2}}>0
$$

It remains to be seen what happens if $1 /(1-R)$ is in the Gumbel domain of attraction, as might be the case if $R$ arises as a Fisher-transformed normal random variable. At present, no general result apart from Proposition 7.4 seems possible: recall that even for the sum or the product of two random variables in the Gumbel domain of attraction, no general results are available on the tail behavior.

An illustration of the finding in this section can be found in Figure 7.3, the left graphs of which shows the penultimate coefficient of tail dependence $\lambda(u)=P[V<u \mid U<u]$ looking at finite but extremely small quantiles $u$ ranging from $10^{-3}$ to $10^{-10}$ and when correlation is random generated by (7.1). It can be seen quite clearly that the penultimate tail dependence is not only quite sizeable even very far in the tail when correlations are random, but that the speed of decline of the tail probability is extremely slow. We want to investigate this speed of decline of $\lambda(u)$ numerically. From (7.10) it follows that

$$
\log \{C(V<u \mid U<u)\} \approx \text { constant }+p \cdot u,
$$


where $p=1-1 / \eta$. The slope $p$ can be estimated by a simple least squares regression and using the relation $\eta=1 /(1-p)$ the coefficient of tail dependence $\eta$ can be recovered. We estimate it over intervals $\left[u_{L}, u_{U}\right]=\left[u^{-k}, u^{-k-3}\right]$ for $k$ ranging from 3 to 10 taking a grid of 0.01 . The corresponding estimates of $\eta$ as a function of $u_{L}$ are depicted in the right graph of Figure 7.3. For the constant correlation case $\eta \approx 0.75$, which corresponds to its analytic value, see Ledford and Tawn (1996). On the other hand, when correlation is not constant $\eta$ is rather close to one and seems to be converging to 1 as $u \downarrow 0$.

The results in this section suggest that at finite samples data generated by (7.8) cannot be distinguished from data generated by a copula that has asymptotically dependent tails. Conversely, such a model is likely to provide a good fit to data for which joint exceedances at large quantiles occur, which is the case for many financial time series and one of the main arguments for using copulas that allow for positive tail dependence such as the Clayton or Student $t$-copula.

\subsection{Consequences for Modeling}

In this section we illustrate the consequences of our results for modeling data with static and dynamic $t$-copulas, in particular to infer the degree of dependence in the tails of the distribution and to see how these estimates differ when correlation is falsely assumed to be constant. From our earlier findings it is clear that when ignoring time-varying correlations and estimating a static $t$-copula, one cannot estimate the correlation coefficient, the degrees-of-freedom parameter and the tail dependence coefficient correctly at the same time, and the estimates will depend on the estimator one applies. The estimator we use here is a combination of maximum likelihood (to estimate $\nu$ ) and the method of moments (to estimate $\rho$ ). The likelihood function for $\nu$ is given by

$$
L\left(\nu \mid U_{1}, U_{2}\right)=\mathbf{t}_{\nu}\left(t_{\nu}^{-1}\left(U_{1}\right), t_{\nu}^{-1}\left(U_{2}\right), \hat{\rho}\right)
$$

where $\mathbf{t}$ denotes the density of the bivariate $t$-distribution and $\hat{\rho}=\operatorname{corr}\left(t_{\nu}^{-1}\left(U_{1}\right), t_{\nu}^{-1}\left(U_{2}\right)\right)$.

\subsubsection{Simulation Study}

Here we present a short Monte Carlo study on the ability of static $t$-copulas to estimate penultimate coefficient of tail dependence $\lambda(u)$ and the limiting coefficient of tail dependence $\lambda$ when the correlation coefficient falsely is assumed to be constant. We simulate data from a Student $t$-copula with a fixed degrees of freedom parameter $\nu$, but with stochastic correlations. The correlations 


\begin{tabular}{lcccc}
\multicolumn{5}{c}{ Table 7.1 : Bias estimating $\lambda(u)$ and $\lambda$ using $t$-copulas } \\
\hline \hline$\lambda(u)$ & $\sigma=0.05$ & $\sigma=0.1$ & $\sigma=0.15$ & $\sigma=0.2$ \\
$\nu=5$ & -0.011 & -0.001 & 0.002 & -0.022 \\
$\nu=10$ & -0.027 & 0.014 & 0.022 & 0.008 \\
$\nu=20$ & -0.082 & 0.005 & 0.029 & 0.031 \\
$\nu=\infty$ & -0.096 & 0.000 & 0.034 & 0.030 \\
\hline$\lambda$ & & & & \\
$\nu=5$ & 0.002 & 0.029 & 0.029 & 0.012 \\
$\nu=10$ & 0.027 & 0.081 & 0.107 & 0.097 \\
$\nu=20$ & 0.039 & 0.142 & 0.213 & 0.235 \\
$\nu=\infty$ & 0.022 & 0.131 & 0.240 & 0.303 \\
\hline \hline
\end{tabular}

Note: This table shows the average bias for estimating $\lambda(u$ with $u=0.01$ and $\lambda$ implied by a $t$-copula when correlation is assumed to be constant, whereas the data has been generated by $t$-copulas with stochastic correlations as in (7.1). The sample size and the number of Monte Carlo replications are equal to 1000 .

are drawn from the Gaussian autoregressive process of order one in (7.1). We chose $\beta=0.97$, $\sigma=0.05,0.1,0.15,0.2$, whereas $\alpha$ was chosen such that the unconditional correlation is equal to 0.5 . The sample size and the number of Monte Carlo replications were chosen to be equal to 1000 . The degrees-of-freedom parameter $\nu$ ranges over the values $5,10,50$, and $\infty$, the last case corresponding to the Gaussian copula. For each of the 1000 simulated data sets we estimate the parameters of the time-constant $t$-copula $C_{\text {Student }}(U, V \mid \rho, \nu)$ and compute both the implied coefficient of tail dependence using (7.6) and the penultimate tail dependence $\lambda(u)$ for $u=0.01$, so a quantile that can be of practical interest.

Table 7.1 reports the average estimation bias for $\lambda$ and $\lambda(u)$. Overall, $\lambda(u)$ is estimated with much more precision than $\lambda$ and both are estimated best when the degrees-of-freedom parameter is low. For both the bias increases in $\nu$ and for the coefficient of tail dependence it also increases in the volatility of the correlation process $\sigma$. Notably, the estimates suggest quite strong tail dependence even when the data is generated by a Gaussian copula. The unreported estimates of $\rho$ and $\nu$ show that the bias in $\lambda$ is due to very low estimates of $\nu$, whereas $\rho$ is only slightly underestimated with average estimates ranging from 0.48 to 0.5 . We conclude that the degrees-of-freedom parameter $\nu$ is estimated such that the estimated copula matches the penultimate coefficient of tail dependence $\lambda(u)$ quite well at the practically relevant quantile $u=0.01$. Consequently, the tail dependence coefficient $\lambda$ is estimated with a serious bias. Note that when the correlations are driven by other distributions than the Gaussian, when correlations are i.i.d. instead of autoregressive, or when we vary the level of the unconditional correlation these findings continue to hold for various sample sizes. Simulation 
results for such situations are not reported but are available upon request.

\subsubsection{Empirical Study}

We illustrate the consequences of our results above for a number of bivariate data sets by looking at the difference in estimates for $\lambda(u)$ and $\lambda$ between static and dynamic $t$-copulas. We consider international stock market returns at daily and monthly frequency and daily exchange rate returns. The data sets are daily returns of the Dow Jones industrial index (DJ) and the NASDAQ composite index (NQ) from March 26, 1990 until March 23, 2000, daily returns of the MSCI index for France (Fra) and Germany (Ger) from October 6, 1989 until October 17, 2008, monthly returns of the Datastream stock index for Germany, Japan (Jap), the UK and the US from January 1974 until May 2008, and daily exchange rate returns of the Euro (EUR), British pound (GBP) and Japanes Yen (JPY) against the US dollar from January 1, 2005 until December 31, 2008. Returns are calculated as 100 times the first difference of the natural logarithm of prices.

Before we can fit a copula to the data we first need an appropriate model for the marginal distributions. The main feature of the financial data we have to model is heteroscedasticity, which can be captured either by GARCH models introduced by Bollerslev (1986) or stochastic volatility (SV) models proposed by Clark (1973) and Taylor (1986). We opt for the latter models class due to its higher flexibility and better fit, and because of its natural connection to the stochastic copula model for modeling the correlation dynamics. The basic SV model for the return $r_{t}$ for $t=1, \ldots, T$ is given by

$$
\begin{aligned}
& r_{t}=\exp \left(h_{t} / 2\right) \varepsilon_{t} \\
& h_{t}=\alpha+\beta h_{t-1}+\nu \eta_{t},
\end{aligned}
$$

where $\varepsilon_{t}$ and $\eta_{t}$ are mutually independent i.i.d. normal random variables with mean zero and variance one that are also uncorrelated with the innovations driving the dependence process. Estimation of the model is done by simulated maximum likelihood using the efficient importance sampler (EIS) by Liesenfeld and Richard (2003).

The static $t$-copulas are estimated as described above, whereas for the time-varying model we condition the estimate of the degrees-of-freedom parameter conditional on time-varying correlations estimated using the SCAR model by Hafner and Manner (2008) giving estimates $\hat{\rho}_{t}$ and $\hat{\nu} \mid \hat{\rho}_{t}$.

Next to the estimated degree-of-freedom parameters $\hat{\nu}$ and $\hat{\nu} \mid \hat{\rho}_{t}$ we report the implied coefficient 
of tail-dependence $\lambda$ and the penultimate coefficient of tail dependence $\lambda(u)$ at various economically interesting quantiles. In particular, the quantiles we consider correspond to exceedances that are expected to occur once a year, once a decade and once a century, which are consequently different for monthly and daily data and are calculated as one divided by the number of observations in the corresponding time interval. We denote the corresponding estimates by $\lambda_{\text {year }}, \lambda_{\text {dec }}$ and $\lambda_{\text {cent }}$. These can be interpreted as the probability that one market makes an extreme downward movement conditional on a significant drop in the other. The coefficient of tail dependence $\lambda$, on the other hand, denotes the probability of one market crashing completely, i.e. dropping to a level of zero, conditional on the other market crashing. This is an economically rather unrealistic situation and is, in our view, only relevant when considering individual stocks, since a single company can go bankrupt and contagion effects can cause similar companies to go bankrupt as well.

The results can be found in Table 7.2. The most striking finding is that the estimated degrees-offreedom parameter is significantly larger when correlations are stochastic in many cases being virtually infinity $^{2}$. This implies that a large part of the fat-tailedness can be captured by random correlations. Looking at the measures of dependence in the tails two things are notable. First, the dependence at larger quantiles is much larger than the limiting coefficient of tail dependence. This suggests that looking at $\lambda$ to assess the risk of crisis spillovers will underestimate the probability of a crash in one market conditional on a crash in another market. Second, although the models using timevarying correlations have a much lower limiting coefficient of tail dependence, at practically relevant quantiles these models capture the dependence in the tails of the distribution quite well. These findings correspond to our simulation results that both models seem to be able to match penultimate tail dependence, but that static $t$-copulas may overestimate the coefficient of tail dependence when the tail are not too heavy.

\subsection{Conclusions}

We have studied the dependence in the tails of elliptical copulas with time-varying parameters. We have shown that the (penultimate) coefficient of tail dependence of time-varying elliptical copulas is larger than that of static ones with the same unconditional correlation. Furthermore, we found that penultimate coefficient of tail dependence of $t$-copulas, i.e. the tail dependence at sub-asymptotic

\footnotetext{
${ }^{2}$ We set the upper bound of the optimization routine equal to 400 and report $\infty$ whenever the upper bound was obtained.
} 
Table 7.2: Finite level and asymptotic tail dependence of static and dynamic $t$-copulas

\begin{tabular}{lccccccccccc}
\hline \hline & \multicolumn{1}{c}{ Static correlations } & \multicolumn{1}{c}{ Dynamic correlations } \\
Data & $\hat{\nu}$ & $\lambda_{\text {year }}$ & $\lambda_{\text {dec }}$ & $\lambda_{\text {cent }}$ & $\lambda$ & $\hat{\nu} \mid \hat{\rho}_{t}$ & $\lambda_{\text {year }}$ & $\lambda_{\text {dec }}$ & $\lambda_{\text {cent }}$ & $\lambda$ \\
\hline Daily stock market & & & & & & & & & & \\
DJ-NQ & 6.06 & 0.36 & 0.33 & 0.32 & 0.31 & $\infty$ & 0.26 & 0.18 & 0.13 & 0.00 \\
Fra-Ger & 2.89 & 0.51 & 0.50 & 0.50 & 0.50 & 17.51 & 0.30 & 0.25 & 0.22 & 0.21 \\
\hline Monthly stock market & & & & & & & & & & & \\
Ger-Jap & 12.39 & 0.24 & 0.11 & 0.05 & 0.02 & $\infty$ & 0.23 & 0.08 & 0.04 & 0.00 \\
Ger-UK & 6.22 & 0.36 & 0.25 & 0.19 & 0.18 & 15.95 & 0.37 & 0.37 & 0.36 & 0.08 \\
Ger-US & 3.93 & 0.39 & 0.31 & 0.28 & 0.27 & 11.20 & 0.35 & 0.23 & 0.15 & 0.11 \\
Jap-UK & 10.32 & 0.26 & 0.13 & 0.07 & 0.04 & 41.03 & 0.25 & 0.10 & 0.03 & 0.00 \\
Jap-US & 9.91 & 0.26 & 0.13 & 0.07 & 0.05 & $\infty$ & 0.26 & 0.11 & 0.06 & 0.00 \\
UK-US & 14.85 & 0.41 & 0.25 & 0.14 & 0.07 & $\infty$ & 0.47 & 0.43 & 0.42 & 0.00 \\
\hline Exchange rates & & & & & & & & & & \\
EUR-GBP & 8.03 & 0.37 & 0.33 & 0.31 & 0.28 & 39.62 & 0.31 & 0.23 & 0.18 & 0.03 \\
EUR-JPY & 5.51 & 0.20 & 0.18 & 0.17 & 0.16 & $\infty$ & 0.14 & 0.09 & 0.06 & 0.00 \\
GBP-JPY & 4.55 & 0.17 & 0.16 & 0.15 & 0.15 & $\infty$ & 0.21 & 0.16 & 0.13 & 0.00 \\
\hline \hline
\end{tabular}

Note: This table presents estimates of the degrees of freedom of $t$-copula conditional on constant correlation (column 2) and autoregressive correlation of order 1 (column 7 ). The remaining column show the dependence in the tails at finite and asymptotic quantiles. The marginal distributions were Gaussian stochastic volatility models.

quantiles, is always larger than its limit and that the difference between the two can be substantial. Finally, we discovered that under some fairly weak conditions time-varying Gaussian copulas have tails that are much heavier than one would intuitively expect and fall into the newly defined category of 'near asymptotic dependence'. In practice this implies that it is virtually impossible to distinguish such copulas from ones that have asymptotically dependent tails such as $t$-copulas. In a simulation study we found that ignoring the time-variation of correlations when estimating $t$-copulas leads to biased estimates of the coefficient of tail dependence. Our empirical application showed that estimates of the degree-of-freedom parameter of $t$-copula are much lower when assuming static correlation than when conditioning on dynamic correlation. At the same time the models based on dynamic correlations produce similar dependence in the tails at economically relevant quantiles, but lower tail dependence in the limit.

Concluding, our findings suggest that for practical purposes the Gaussian copula is more attractive than often stated in the literature, as long as one accounts for the (empirically observed) time-varying correlations. This can be seen as an analogue to the effect that conditionally Gaussian models for time-varying volatility such as GARCH and stochastic volatility models can create fat tails in the individual series. Thus, conditionally Normal models are more than just a simplifying approximation 
in a multivariate setting, as they are able to capture both fat tails in the marginals and dependence in the tails of the copula.

\section{A Proofs}

Proof of Proposition 7.1. Write $\rho=\sin \gamma \in[-1,1]$ for $\gamma=\arcsin \rho \in\left[-\frac{\pi}{2}, \frac{\pi}{2}\right]$. Then $\left(1-\rho^{2}\right)^{1 / 2}=$ $\cos \gamma \in[0,1]$, so that

$$
\begin{aligned}
Y & =\rho S_{1}+\left(1-\rho^{2}\right)^{1 / 2} S_{2} \\
& =R \sin \gamma \cos \Theta+R \cos \gamma R \sin \Theta=R \sin (\Theta+\gamma),
\end{aligned}
$$

yielding the representation

$$
(X, Y)=(R \cos \Theta, R \sin (\Theta+\gamma))
$$

Let $t \geqslant 0$ be such that $1-F(t)=F(-t)=u$, where $F$ is the marginal distribution function of $X$ and $Y$. Since the distribution of $(X, Y)$ is symmetric around zero and since $R$ and $\Theta$ are independent,

$$
\begin{aligned}
C_{\rho}(u, u) & =\mathrm{P}(X>t, Y>t) \\
& =\mathrm{P}[\cos \Theta>t / R, \sin (\Theta+\gamma)>t / R] \\
& =\int_{t}^{\infty} \mathrm{P}\left[\cos \Theta>\frac{t}{r}, \sin (\Theta+\gamma)>\frac{t}{r}\right] \mathrm{d} F_{R}(r),
\end{aligned}
$$

with $F_{R}$ the distribution function of $R$. As a consequence, it is sufficient to show that for fixed $z \in[0,1)$, the function

$$
\rho \mapsto \mathrm{P}[\cos \Theta>z, \sin (\Theta+\gamma)>z]
$$

is convex in $\rho \in[0,1]$, where $\gamma=\arcsin \rho \in\left[0, \frac{\pi}{2}\right]$ and with $\Theta$ uniformly distributed on $(-\pi, \pi)$. Write $z=\cos \alpha \in[0,1)$, so $\alpha=\arccos z \in(0, \pi]$. Then

$$
\begin{array}{r}
\cos \Theta>\cos \alpha \\
\Longleftrightarrow-\alpha<\Theta<\alpha
\end{array}
$$


whereas

$$
\begin{aligned}
& \sin (\Theta+\gamma)>\cos \alpha=\sin \left(\frac{\pi}{2}-\alpha\right) \\
\Longleftrightarrow & \frac{\pi}{2}-\alpha<\Theta+\gamma<\frac{\pi}{2}+\alpha .
\end{aligned}
$$

Joining these two double inequalities and using the fact that $\frac{\pi}{2}-\gamma \geqslant 0$ yields

$$
\mathrm{P}[\cos \Theta>z, \sin (\Theta+\gamma)>z]=\mathrm{P}\left(-\alpha+\frac{\pi}{2}-\gamma<\Theta<\alpha\right)=\frac{1}{2 \pi} \max \left(2 \alpha-\frac{\pi}{2}+\gamma, 0\right)
$$

Since $\gamma=\arcsin \rho$ is convex in $\rho \in[0,1]$, the result follows.

Proof of Proposition 7.2. We keep the same notations as in the proof of Proposition 7.1. Now let $0<u<1 / 2$ so that $t=F^{-1}(1-u)>0$. We have

$$
C_{\rho}(u, u)=\int_{t}^{\infty} \frac{1}{2 \pi} \max \left(2 \alpha-\frac{\pi}{2}+\gamma, 0\right) \mathrm{d} F_{R}(r)
$$

where $\alpha=\arccos (t / r)$ and $\gamma=\gamma(\rho)=\arcsin \rho$. Observe that

$$
\begin{gathered}
2 \alpha-\frac{\pi}{2}+\gamma>0 \\
\Longleftrightarrow r>\frac{t}{\cos \left(\left(\frac{\pi}{2}-\gamma\right) / 2\right)}=r(\rho) .
\end{gathered}
$$

We find

$$
2 \pi \frac{\mathrm{d}}{\mathrm{d} \rho} C_{\rho}(u, u)=\bar{F}_{R}(r(\rho)) \frac{\mathrm{d} \gamma(\rho)}{\mathrm{d} \rho}
$$

and

$$
2 \pi \frac{\mathrm{d}^{2}}{\mathrm{~d} \rho^{2}} C_{\rho}(u, u)=-f_{R}(r(\rho)) \frac{\mathrm{d} r(\rho)}{\mathrm{d} \rho} \frac{\mathrm{d} \gamma(\rho)}{\mathrm{d} \rho}+\bar{F}_{R}(r(\rho)) \frac{\mathrm{d}^{2} \gamma(\rho)}{\mathrm{d} \rho^{2}}
$$

We have

$$
\begin{gathered}
\frac{\mathrm{d} \gamma(\rho)}{\mathrm{d} \rho}=\left(1-\rho^{2}\right)^{-1 / 2}, \\
\frac{\mathrm{d}^{2} \gamma(\rho)}{\mathrm{d} \rho^{2}}=\frac{\rho}{1-\rho^{2}} \frac{\mathrm{d} \gamma(\rho)}{\mathrm{d} \rho} .
\end{gathered}
$$


Some goniometric juggling yields

$$
\begin{aligned}
\cos \left(\left(\frac{\pi}{2}-\gamma\right) / 2\right) & =\left(\frac{1+\cos \left(\frac{\pi}{2}-\gamma\right)}{2}\right)^{1 / 2} \\
& =\left(\frac{1+\sin \gamma}{2}\right)^{1 / 2}=\left(\frac{1+\rho}{2}\right)^{1 / 2}
\end{aligned}
$$

whence

$$
\begin{aligned}
r(\rho) & =2^{1 / 2} t(1+\rho)^{-1 / 2}, \\
\frac{\mathrm{d} r(\rho)}{\mathrm{d} \rho} & =-\frac{1}{2} \frac{1}{1+\rho} r(\rho) .
\end{aligned}
$$

Writing

$$
\nu(r)=\frac{r f_{R}(r)}{\bar{F}(r)}
$$

we conclude that

$$
2 \pi \frac{\mathrm{d}^{2}}{\mathrm{~d} \rho^{2}} C_{\rho}(u, u)=\bar{F}(r(\rho)) \frac{\mathrm{d} \gamma(\rho)}{\mathrm{d} \rho} \frac{1}{1+\rho}\left(\frac{1}{2} \nu(r(\rho))+\frac{\rho}{1-\rho}\right)
$$

For $\rho<0$, we have $r(\rho)>t$. If $u$ is small enough so that $t$ is large enough so that $\nu(r)>1$ for all $r \geqslant t$, then the factor between big brackets on the right-hand side of the last display is positive for all $\rho \in(-1,1)$. Hence $C_{\rho}(u, u)$ is convex in $\rho$.

Proof of Proposition 7.3. Let $x>0$. We have

$$
\begin{aligned}
\mathrm{P}(X>x, Y>x) & =\mathrm{P}\left(S^{-1} Z_{1}>x, S^{-1}\left(\rho Z_{1}+\sqrt{1-\rho^{2}} Z_{2}\right)>x\right) \\
& =\mathrm{P}\left(\min \left(Z_{1}, \rho Z_{1}+\sqrt{1-\rho^{2}} Z_{2}\right) S^{-1}>x\right) .
\end{aligned}
$$

Put $W=\min \left(Z_{1}, \rho Z_{1}+\sqrt{1-\rho^{2}} Z_{2}\right)$ and $W_{+}=\max (W, 0)$. Then we can rewrite the above equation as

$$
\mathrm{P}(X>x, Y>x)=\mathrm{P}\left(W_{+} S^{-1}>x\right) .
$$

We need precise information on the upper tail of $S^{-1}$ : see Lemma 7.1. We can then proceed as in 
Breiman's lemma:

$$
\begin{aligned}
\mathrm{P}(X>x, Y>x) & =\int_{(0, \infty)} \mathrm{P}\left(S^{-1}>x / w\right) d \mathrm{P}(W \leqslant w) \\
& =\int_{(0, \infty)} a_{\nu}(x / w)^{-\nu}\left(1-b_{\nu}(x / w)^{-2}\right) d \mathrm{P}(W \leqslant w)+\int_{(0, \infty)} R(x / w) d \mathrm{P}(W \leqslant w) \\
& =a_{\nu} x^{-\nu}\left(\mathrm{E}\left[W_{+}^{\nu}\right]-b_{\nu} x^{-2} \mathrm{E}\left[W_{+}^{\nu+2}\right]\right)+\int_{(0, \infty)} R(x / w) d \mathrm{P}(W \leqslant w) .
\end{aligned}
$$

The remainder term is

$$
0 \leqslant \int_{(0, \infty)} R(x / w) d \mathrm{P}(W \leqslant w) \leqslant c_{\gamma} x^{-\nu-4} \mathrm{E}\left[W_{+}^{\nu+4}\right]
$$

We find that

$$
\mathrm{P}(X>x, Y>x)=a_{\nu} x^{-\nu}\left(\mathrm{E}\left[W_{+}^{\nu}\right]-b_{\nu} x^{-2} \mathrm{E}\left[W_{+}^{\nu+2}\right]+O\left(x^{-4}\right)\right), \quad x \rightarrow \infty .
$$

The marginal tail of $X$ can be represented in the same way: it suffices to replace $W$ in the preceding display by a standard normal random variable $Z$, that is,

$$
\mathrm{P}(X>x)=\mathrm{P}\left(S^{-1} Z>x\right)=a_{\nu} x^{-\nu}\left(\mathrm{E}\left[Z_{+}^{\nu}\right]-b_{\nu} x^{-2} \mathrm{E}\left[Z_{+}^{\nu+2}\right]+O\left(x^{-4}\right)\right), \quad x \rightarrow \infty .
$$

It follows that as $x \rightarrow \infty$,

$$
\begin{aligned}
\mathrm{P}(Y>x \mid X>x) & =\frac{\mathrm{P}(X>x, Y>x)}{\mathrm{P}(X>x)} \\
& =\frac{\mathrm{E}\left[W_{+}^{\nu}\right]-b_{\nu} x^{-2} \mathrm{E}\left[W_{+}^{\nu+2}\right]+O\left(x^{-4}\right)}{\mathrm{E}\left[Z_{+}^{\nu}\right]-b_{\nu} x^{-2} \mathrm{E}\left[Z_{+}^{\nu+2}\right]+O\left(x^{-4}\right)} \\
& =\frac{\mathrm{E}\left[W_{+}^{\nu}\right]}{\mathrm{E}\left[Z_{+}^{\nu}\right]}+b_{\nu} x^{-2} \frac{\mathrm{E}\left[W_{+}^{\nu}\right] \mathrm{E}\left[Z_{+}^{\nu+2}\right]-\mathrm{E}\left[W_{+}^{\nu+2}\right] \mathrm{E}\left[Z_{+}^{\nu}\right]}{\left(\mathrm{E}\left[Z_{+}^{\nu}\right]\right)^{2}}+O\left(x^{-4}\right) .
\end{aligned}
$$

As a consequence, the coefficient of tail dependence $\lambda=\lim _{x \rightarrow \infty} \mathrm{P}(Y>x \mid X>x)$ is given as in (7.7).

Moreover, the expansion in (7.17) gives us a handle on the rate of convergence of $C(u, u) / u$ towards $\lambda$. Let $t_{\nu}^{-1}$ be the quantile function of the $t$-distribution with $\nu$ degrees of freedom. By 
symmetry of the upper and lower tails,

$$
\begin{aligned}
\frac{C(u, u)}{u} & =\mathrm{P}\left(Y>t_{\nu}^{-1}(1-u) \mid X>t_{\nu}^{-1}(1-u)\right) \\
& =\lambda+b_{\nu} \frac{\mathrm{E}\left[W_{+}^{\nu}\right] \mathrm{E}\left[Z_{+}^{\nu+2}\right]-\mathrm{E}\left[W_{+}^{\nu+2}\right] \mathrm{E}\left[Z_{+}^{\nu}\right]}{\left(\mathrm{E}\left[Z_{+}^{\nu}\right]\right)^{2}}\left(t_{\nu}^{-1}(1-u)\right)^{-2}+O\left(\left(t_{\nu}^{-1}(1-u)\right)^{-4}\right)
\end{aligned}
$$

Since $1-t_{\nu}(x)=\mathrm{P}(X>x) \sim a_{\nu} x^{-\nu} \mathrm{E}\left[Z_{+}^{\nu}\right]$ as $x \rightarrow \infty$, we have

$$
t_{\nu}^{-1}(1-u) \sim\left(a_{\nu} \mathrm{E}\left[Z_{+}^{\nu}\right]\right)^{1 / \nu} u^{-1 / \nu}, \quad u \downarrow 0 .
$$

(By $f(y) \sim g(y)$ we mean that $f(y) / g(y) \rightarrow 1$.) We obtain that

$$
\frac{C(u, u)}{u}=\lambda+\gamma u^{2 / \nu}+o\left(u^{2 / \nu}\right)
$$

where

$$
\gamma=b_{\nu} \frac{\mathrm{E}\left[W_{+}^{\nu}\right] \mathrm{E}\left[Z_{+}^{\nu+2}\right]-\mathrm{E}\left[W_{+}^{\nu+2}\right] \mathrm{E}\left[Z_{+}^{\nu}\right]}{\left(\mathrm{E}\left[Z_{+}^{\nu}\right]\right)^{2}}\left(a_{\nu} \mathrm{E}\left[Z_{+}^{\nu}\right]\right)^{-2 / \nu} .
$$

Equating (7.7) and (7.6) (for non-random $\rho$ ) we find that

$$
\mathrm{E}\left[W_{+}^{\nu}\right]=\mathrm{E}\left[Z_{+}^{\nu}\right] \mathrm{E}[\lambda(\nu, \rho)]
$$

the latter expectation being with respect to the random variable $\rho$. As a consequence,

$$
\frac{\mathrm{E}\left[W_{+}^{\nu}\right] \mathrm{E}\left[Z_{+}^{\nu+2}\right]-\mathrm{E}\left[W_{+}^{\nu+2}\right] \mathrm{E}\left[Z_{+}^{\nu}\right]}{\left(\mathrm{E}\left[Z_{+}^{\nu}\right]\right)^{2}}=\frac{\mathrm{E}\left[Z_{+}^{\nu+2}\right]}{\mathrm{E}\left[Z_{+}^{\nu}\right]} \mathrm{E}[\lambda(\nu, \rho)-\lambda(\nu+2, \rho)],
$$

the sign of which is positive [since $\lambda(\nu, \rho)$ is decreasing in $\nu$ ]. Furthermore, by (7.20) and the identity $\Gamma(z+1)=z \Gamma(z)$,

$$
\frac{\mathrm{E}\left[Z_{+}^{\nu+2}\right]}{\mathrm{E}\left[Z_{+}^{\nu}\right]}=\frac{\frac{2^{\nu / 2}}{\sqrt{\pi}} \Gamma((\nu+3) / 2)}{\frac{2^{\nu / 2-1}}{\sqrt{\pi}} \Gamma((\nu+1) / 2)}=2 \frac{\nu+1}{2}=\nu+1
$$

We obtain

$$
\gamma=\left(a_{\nu} \mathrm{E}\left[Z_{+}^{\nu}\right]\right)^{-2 / \nu} b_{\nu}(\nu+1) \mathrm{E}[\lambda(\nu, \rho)-\lambda(\nu+2, \rho)]
$$

with $a_{\nu}$ and $b_{\nu}$ given in (7.19) and $\mathrm{E}\left[Z_{+}^{\nu}\right]$ in (7.20) below.

Lemma 7.1. For $\nu>0$, let $S$ be a positive random variable such that $\nu S^{2}$ is chi-square distribution 
with degrees of freedom $\nu$. Then for all $z>0$,

$$
\mathrm{P}\left(S^{-1}>z\right)=a_{\nu} z^{-\nu}\left(1-b_{\nu} z^{-2}\right)+R(z)
$$

where

$$
a_{\nu}=\frac{(\nu / 2)^{\nu / 2}}{\Gamma(\nu / 2+1)}, \quad b_{\nu}=\frac{(\nu / 2)^{2}}{\nu / 2+1}
$$

and $0 \leqslant R(z) \leqslant c_{\gamma} z^{-\nu-4}$ for some positive constant $c_{\nu}$.

Proof. Since $\nu S^{2}$ is chi-squared with $\nu$ degrees of freedom,

$$
\begin{aligned}
\mathrm{P}\left(S^{-1}>z\right) & =\mathrm{P}\left(S<z^{-1}\right)=\mathrm{P}\left(\nu S^{2}<\nu z^{-2}\right) \\
& =\int_{0}^{\nu z^{-2}} \frac{1}{\Gamma(\nu / 2) 2^{\nu / 2}} y^{\nu / 2-1} e^{-y / 2} d y .
\end{aligned}
$$

For $z \geqslant 0$, we have $e^{-z}-(1-z)=\int_{0}^{z}\left(1-e^{-y}\right) d y=\int_{0}^{z} \int_{0}^{y} e^{-x} d x d y$ and thus $0 \leqslant e^{-z}-(1-z) \leqslant z^{2} / 2$.

We find that

$$
\begin{aligned}
0 & \leqslant \mathrm{P}\left(S^{-1}>z\right)-\frac{1}{\Gamma(\nu / 2) 2^{\nu / 2}} \int_{0}^{\nu z^{-2}} y^{\nu / 2-1}(1-y / 2) d y \\
& \leqslant \frac{1}{\Gamma(\nu / 2) 2^{\nu / 2}} \int_{0}^{\nu z^{-2}} y^{\nu / 2-1} \frac{(y / 2)^{2}}{2} d y=\text { constant } z^{-\nu-4}
\end{aligned}
$$

the constant depending on $\nu$. The integral can be computed as follows:

$\frac{1}{\Gamma(\nu / 2) 2^{\nu / 2}} \int_{0}^{\nu z^{-2}} y^{\nu / 2-1}(1-y / 2) d y=\frac{1}{\Gamma(\nu / 2) 2^{\nu / 2}}\left(\frac{\left(\nu z^{-2}\right)^{\nu / 2}}{\nu / 2}-\frac{1}{2} \frac{\left(\nu z^{-2}\right)^{\nu / 2+1}}{\nu / 2+1}\right)=a_{\nu} z^{-\nu}\left(1-b_{\nu} z^{-2}\right)$

where

$$
\begin{aligned}
a_{\nu} & =\frac{1}{\Gamma(\nu / 2) 2^{\nu / 2}} \frac{\nu^{\nu / 2}}{\nu / 2}=\frac{(\nu / 2)^{\nu / 2}}{\Gamma(\nu / 2+1)} \\
b_{\nu} & =\frac{1}{2} \frac{\nu^{\nu / 2+1}}{\nu / 2+1} / \frac{\nu^{\nu / 2}}{\nu / 2}=\frac{(\nu / 2)^{2}}{\nu / 2+1} .
\end{aligned}
$$


Lemma 7.2. For $\nu>0$ and $Z$ standard normal,

$$
E\left[Z_{+}^{\nu}\right]=\frac{2^{\nu / 2-1}}{\sqrt{\pi}} \Gamma((\nu+1) / 2)
$$

Proof. Substituting $y=z^{2} / 2$, we have

$$
\begin{aligned}
\mathrm{E}\left[Z_{+}^{\nu}\right] & =\frac{1}{\sqrt{2 \pi}} \int_{0}^{\infty} z^{\nu} e^{-z^{2} / 2} d z \\
& =\frac{1}{\sqrt{2 \pi}} \int_{0}^{\infty}(2 y)^{(\nu-1) / 2} e^{-y} d z=\frac{2^{(\nu-1) / 2}}{\sqrt{2 \pi}} \Gamma((\nu+1) / 2) .
\end{aligned}
$$

Lemma 7.3. Let $-1<\rho<1$, let $Z_{1}$ and $Z_{2}$ be independent standard normal random variables, and put $W=\min \left(Z_{1}, \rho Z_{1}+\sqrt{1-\rho^{2}} Z_{2}\right)$. For $\nu>0$, we have

$$
E\left[W_{+}^{\nu}\right]=\frac{2^{\nu / 2}}{\sqrt{\pi}} \Gamma((\nu+1) / 2) t_{\nu+1}(-\sqrt{\nu+1} \sqrt{1-\rho} / \sqrt{1+\rho})
$$

where $t_{\mu}$ is the cumulative distribution function of the t-distribution with degrees of freedom $\mu$.

Proof. Equating (7.7) and (7.6) (for non-random $\rho$ ) we find that

$$
\mathrm{E}\left[W_{+}^{\nu}\right]=\mathrm{E}\left[Z_{+}^{\nu}\right] \lambda(\nu, \rho)
$$

In view of (7.20), the result follows.

Proof of Proposition 7.4. From (7.9), we already know that $C(u, u) \ll u$ as $u \downarrow 0$. So fix $\varepsilon>0$. We have to prove that $u^{1+\varepsilon} \ll C(u, u)$ as $u \downarrow 0$.

The pair $\left(X_{1}, X_{2}\right)$ admits the representation

$$
\left(X_{1}, X_{2}\right) \stackrel{d}{=}\left(X, R X+\sqrt{1-R^{2}} Y\right)
$$

where $X, Y, R$ are independent random variables and $X$ and $Y$ are standard normal. Fix $0<\rho<1$. 
By symmetry, if $0<u<1 / 2$,

$$
\begin{aligned}
C(u, u) & =P\left[X_{1}>\Phi^{-1}(1-u), X_{2}>\Phi^{-1}(1-u)\right] \\
& =P\left[X>\Phi^{-1}(1-u), R X+\sqrt{1-R^{2}} Y>\Phi^{-1}(1-u)\right] \\
& \geqslant P\left[X>\Phi^{-1}(1-u), \rho X>\Phi^{-1}(1-u), R>\rho, Y>0\right] \\
& =\frac{1}{2} P[R>\rho] P\left[X>\Phi^{-1}(1-u) / \rho\right] .
\end{aligned}
$$

Let $\varphi(x)=(2 \pi)^{-1 / 2} \exp \left(-x^{2} / 2\right)$ denote the standard normal density function and write $\bar{\Phi}=1-\Phi$.

From Mill's ratio,

$$
\lim _{x \rightarrow \infty} \frac{\varphi(x)}{x \bar{\Phi}(x)}=1
$$

it follows $\Phi^{-1}(1-u) \sim(-2 \log u)^{1 / 2}$ as $u \downarrow 0$ and therefore

$$
\begin{aligned}
& P\left[X>\Phi^{-1}(1-u) / \rho\right]=\bar{\Phi}\left(\Phi^{-1}(1-u) / \rho\right) \\
& \quad \sim \frac{\varphi\left(\Phi^{-1}(1-u) / \rho\right)}{\Phi^{-1}(1-u) / \rho} \sim \rho(-2 \log u)^{-1 / 2} u^{1 / \rho^{2}} .
\end{aligned}
$$

If $\rho$ is such that $1 / \rho^{2}<1+\varepsilon$, that is, $\rho>(1+\varepsilon)^{-1 / 2}$, then $P\left[X>\Phi^{-1}(1-u) / \rho\right] \gg u^{1+\varepsilon}$, as required.

Proof of Proposition 7.5. We have

$$
\begin{aligned}
P\left[R X+\sqrt{1-R^{2}} Y>x \mid\right. & X>x] \\
=P\left[R X+\sqrt{1-R^{2}} Y>\right. & x, Y \leqslant x \mid X>x] \\
& +P\left[R X+\sqrt{1-R^{2}} Y>x, Y>x \mid X>x\right]
\end{aligned}
$$

The second term is bounded by $P[Y>x]=\bar{\Phi}(x)=o\left(G\left(x^{2}\right)\right)$. From Lemma 7.4 below, the first probability on the right-hand side of the previous display can be written as

$$
\begin{aligned}
& P\left[R X+\sqrt{1-R^{2}} Y>x, Y \leqslant x \mid X>x\right] \quad=P\left[R>r_{0}(X, Y, x), Y \leqslant x \mid X>x\right],
\end{aligned}
$$


with $r_{0}(X, Y, x)$ as in (7.21). Now write

$$
Z_{x}=x(X-x)
$$

The density of $Z_{x}$ given $X>x$ is the function $f_{x}$ in Lemma 7.5 below. By independence of $R, Y, Z_{x}$, we obtain

$$
\begin{aligned}
P[R X & \left.+\sqrt{1-R^{2}} Y>x, Y \leqslant x \mid X>x\right] \\
& =P\left[R>r_{0}\left(x+Z_{x} / x, Y, x\right), Y \leqslant x\right] \\
& =\int_{0}^{\infty} \int_{-\infty}^{x} P\left[R>r_{0}(x+z / x, y, x)\right] \varphi(y) d y f_{x}(z) d z .
\end{aligned}
$$

We have

$$
\begin{aligned}
& 1-r_{0}(X, Y, x)=\frac{X^{2}+Y^{2}-x X+\sqrt{X^{2}-x^{2}+Y^{2}} Y}{X^{2}+Y^{2}} \\
& =\frac{X(X-x)+Y^{2}+\sqrt{X^{2}-x^{2}+Y^{2}} Y}{X^{2}+Y^{2}} .
\end{aligned}
$$

and therefore

$$
\begin{aligned}
1-r_{0}(x+z / x, y, x)=\frac{\left(x+\frac{z}{x}\right) \frac{z}{x}+y^{2}+y \sqrt{\frac{z}{x}\left(2 x+\frac{z}{x}\right)+y^{2}}}{\left(x+\frac{z}{x}\right)^{2}+y^{2}} & \\
& =\frac{z+\frac{z^{2}}{x^{2}}+y^{2}+y \sqrt{2 z+\frac{z^{2}}{x^{2}}+y^{2}}}{x^{2}+2 z+\frac{z^{2}}{x^{2}}+y^{2}} .
\end{aligned}
$$

We have $P(R>r)=G\left(\frac{1}{1-r}\right)$ and thus

$$
\begin{aligned}
& P\left[R X+\sqrt{1-R^{2}} Y>x, Y \leqslant x \mid X>x\right] \\
& =\int_{0}^{\infty} \int_{-\infty}^{x} G\left(\frac{1}{1-r_{0}(x+z / x, y, x)}\right) \varphi(y) d y f_{x}(z) d z \\
& =\int_{0}^{\infty} \int_{-\infty}^{x} G\left(\frac{x^{2}+2 z+\frac{z^{2}}{x^{2}}+y^{2}}{z+\frac{z^{2}}{x^{2}}+y^{2}+y \sqrt{2 z+\frac{z^{2}}{x^{2}}+y^{2}}}\right) \varphi(y) d y f_{x}(z) d z .
\end{aligned}
$$


If interchanging limits and integrals is permitted, then

$$
\begin{aligned}
\lim _{x \rightarrow \infty} \frac{1}{G\left(x^{2}\right)} P\left[R X+\sqrt{1-R^{2}} Y>x, Y \leqslant x \mid X>x\right] & \\
=\int_{0}^{\infty} \int_{-\infty}^{\infty}\left(z+y^{2}+y \sqrt{2 z+y^{2}}\right)^{\alpha} \varphi(y) d y e^{-z} d z & =E\left[\left(Z+Y^{2}+Y \sqrt{2 Z+Y^{2}}\right)^{\alpha}\right]
\end{aligned}
$$

with $Y$ and $Z$ independent random variables, $Y$ standard normal and $Z$ unit exponential. Hence we find (7.12), as required.

How to justify that the limit may be passed through the double integral? The integral may be cut off on the complement of $\left\{y: y^{2} \leqslant x\right\} \times\{z: 0<z \leqslant x\}$, the integral over the complement being bounded by

$$
P\left[Y^{2}>x\right]+P\left[Z_{x}>x\right] \leqslant 2\left(\bar{\Phi}(\sqrt{x})+e^{-x}\right)=o\left(x^{-m}\right), \quad x \rightarrow \infty,
$$

for every $m>0$. On the region $\left\{y: y^{2} \leqslant x\right\} \times\{z: 0<z \leqslant x\}$, the argument of the function $G$ is bounded below by $c x$ for some constant $c>0$. Hence Potter's theorem becomes applicable, yielding an integrable upper bound for the integrand. An application of the dominated convergence theorem yields the desired result.

Finally, to obtain (7.13), it suffices to replace $x$ by $x(u)$ defined as the solution to $P[X>x(u)]=$ $\bar{\Phi}(x(u))=u$. Since $P\left[R>1-x^{-2}\right]=G\left(x^{2}\right)$ and since $x(u)^{2} \sim 2 \log (1 / u)$, regular variation of $G$ yields (7.13).

Lemma 7.4. For $x>0$ and $Y \leqslant x<X$, we have

$$
\begin{aligned}
& \left\{r \in[-1,1]: r X+\sqrt{1-r^{2}} Y>x\right\}=\left(r_{0}, 1\right] \\
& \qquad \text { with } r_{0}=r_{0}(X, Y, x)=\frac{x X-\sqrt{X^{2}-x^{2}+Y^{2}} Y}{X^{2}+Y^{2}} \in(0,1) .
\end{aligned}
$$

Proof. We need to analyse the function

$$
f(r)=r X+\sqrt{1-r^{2}} Y, \quad-1 \leqslant r \leqslant 1 .
$$

Given $x>0$ such that $Y \leqslant x<X$, we want to determine the region $\{r: f(r)>x\}$. 
For $r \leqslant 0$, we have $f(r) \leqslant \sqrt{1-r^{2}} Y \leqslant x$. Hence $f(r)>x$ implies $r>0$. Note that

$$
f(0)=Y \leqslant x, \quad f(1)=X>x .
$$

The derivatives of $f$ are

$$
\begin{aligned}
f^{\prime}(r) & =X-\frac{r}{\sqrt{1-r^{2}}} Y \\
f^{\prime \prime}(r) & =-\frac{\sqrt{1-r^{2}}+\frac{r^{2}}{\sqrt{1-r^{2}}}}{1-r^{2}}=-\frac{\left(1-r^{2}\right)+r^{2}}{\left(1-r^{2}\right)^{3 / 2}} Y=-\frac{1}{\left(1-r^{2}\right)^{3 / 2}} Y
\end{aligned}
$$

Note that $f^{\prime}(0)=X>x>0$.

If $Y>0$, then $f^{\prime \prime}<0$, so that the function $f$ is concave, in which case that the region $\{r: f(r)>$ $x\}$ is an interval. Since this interval includes 1 but does not include 0 , it follows that there exists a unique $0 \leqslant r_{0}=r_{0}(X, Y, x)<1$ such that

$$
f\left(r_{0}\right)=x, \quad\{r: f(r)>x\}=\left\{r: r_{0}<r \leqslant 1\right\} .
$$

If $Y \leqslant 0$, then $f^{\prime \prime} \geqslant 0$, so that the function $f$ is convex. Since $f^{\prime}(0)=X>x>0$, it follows that $f$ is increasing on $[0,1]$. Hence again there must exist a unique $0 \leqslant r_{0}<1$ such that the equations in the above display are true.

So it remains to find $r_{0}$ such that $0 \leqslant r_{0}<1$ and such that $f\left(r_{0}\right)=x$. The equation $f(r)=x$ implies $(r X-x)^{2}=\left(1-r^{2}\right) Y^{2}$ and thus

$$
\left(X^{2}+Y^{2}\right) r^{2}-2 x X r+\left(x^{2}-Y^{2}\right)=0 .
$$

This is a quadratic equation to be solved in $r$. The solutions have been identified in previous calculations as

$$
r_{ \pm}=\frac{x X \pm \sqrt{X^{2}-x^{2}+Y^{2}} Y}{X^{2}+Y^{2}} .
$$

If $Y=0$ then $r_{ \pm}=x / X=r_{0}$. If $Y \neq 0$, however, $r_{-} \neq r_{+}$, so only one of these two values can be equal to $r_{0}$.

If $Y<0$, then the equation $f\left(r_{0}\right)=x$ also implies $r_{0} X>f\left(r_{0}\right)=x$ and thus $r_{0}>x / X$. But if 
$Y<0$ then also

$$
r_{+}<\frac{x X}{X^{2}+Y^{2}}<\frac{x X}{X^{2}}=\frac{x}{X}, \quad \quad r_{-}>\frac{x X+Y^{2}}{X^{2}+Y^{2}}>\frac{x X}{X^{2}}=\frac{x}{X},
$$

whence $r_{0}=r_{-}$.

If $0<Y<x$, then $r_{0} X<f\left(r_{0}\right)=x$ and

$$
r_{-}<\frac{x X}{X^{2}}=\frac{x}{X}<1, \quad r_{-}>\frac{x X-X Y}{X^{2}+Y^{2}}>0, \quad r_{+}>\frac{x X+Y^{2}}{X^{2}+Y^{2}}>\frac{x X}{X^{2}}=\frac{x}{X}
$$

so that again $r_{0}=r_{-}$.

Lemma 7.5. Let $X$ be standard normal. For $x>0$, the conditional density of $x(X-x)$ given $X>x$ evaluated at $u>0$ is

$$
f_{x}(u)=\frac{\varphi(x)}{x \bar{\Phi}(x)} \exp \left(-u-\frac{1}{2} \frac{u^{2}}{x^{2}}\right) .
$$

In particular, $f_{x}(u) \leqslant\left(1+x^{-1}\right) \exp (-u)$ for $x \geqslant 1$ and $\lim _{x \rightarrow \infty} f_{x}(u)=\exp (-u)$.

Proof. We have

$$
\begin{aligned}
f_{x}(u) & =\frac{d}{d u} P[x(X-x) \leqslant u \mid X>x]=\frac{d}{d u} \frac{P\left[x<X \leqslant x+\frac{u}{x}\right]}{P[X>x]} \\
& =\frac{d}{d u} \frac{\Phi\left(x+\frac{u}{x}\right)-\Phi(x)}{1-\Phi(x)}=\frac{\varphi\left(x+\frac{u}{x}\right)}{x \bar{\Phi}(x)}=\frac{\varphi(x)}{x \bar{\Phi}(x)} \frac{\varphi\left(x+\frac{u}{x}\right)}{\varphi(x)} \\
& =\frac{\varphi(x)}{x \bar{\Phi}(x)} \exp \left\{-\frac{1}{2}\left(x+\frac{u}{x}\right)-x^{2}\right\} \\
& =\frac{\varphi(x)}{x \bar{\Phi}(x)} \exp \left(-u-\frac{1}{2} \frac{u^{2}}{x^{2}}\right),
\end{aligned}
$$

as required. If $x \geqslant 1$, then by Mill's ratio

$$
1 \leqslant \frac{\varphi(x)}{x \bar{\Phi}(x)} \leqslant 1+\frac{1}{x}
$$

which converges to unity if $x \rightarrow \infty$. 
Proof of Addendum 7.1. For $z>0$ and $y \in \mathbb{R}$,

$$
\begin{aligned}
z+y^{2}+y \sqrt{2 z+y^{2}} & =z+y \frac{\left(2 z+y^{2}\right)-y^{2}}{\sqrt{2 z+y^{2}}-y}=z+\frac{2 z y}{\sqrt{2 z+y^{2}}-y} \\
& =z \frac{\sqrt{2 z+y^{2}}-y+2 y}{\sqrt{2 z+y^{2}}-y}=z \frac{\sqrt{2 z+y^{2}}+y}{\sqrt{2 z+y^{2}}-y} \\
& =\frac{2 z^{2}}{\left(\sqrt{2 z+y^{2}}-y\right)^{2}},
\end{aligned}
$$

as required. 


\section{Conclusion}

\subsection{Summary and reflections}

In this thesis we have studied copula models for asymmetric and time-varying dependence.

Chapter 2 introduced copulas, estimation techniques and a selection of goodness-of-fit tests. An application to exchange rate data illustrated the methods. No new results were obtained in this chapter, but it rather served to give the reader an overview on existing standard techniques and to enable him to follow the rest of the thesis.

In Chapter 3 the topic of asymmetric dependence was studied. A conditional version of Kendall's tau was proposed and the null hypothesis of symmetric dependence was tested using pseudo likelihood ratio tests on the parameters of copula models. The parametric route was shown to be superior to the model free approach suggested by Hong et al. (2007), even when the parametric models are misspecified. Some examples showed that asymmetric dependence can be generated by quite distinct nonlinear models and that the suggested tests are able to detect such nonlinearities in a reliable manner. The empirical application showed that not only dependence between stock market returns tends to be asymmetric, but it also confirms findings on macroeconomic data originally studied by Altissimo and Violante (2001).

The aim of Chapter 4 was to introduce time-varying copulas and to juxtapose a number of competing models. Furthermore, methods to test the goodness-of-fit for time-varying models were studied and turned out to work quite well. Among the models studied three stand out and can be recommended: The regime switching model, the model for smoothly changing dependence by Hafner and Reznikova (2008) and the stochastic copula model that was studied in detail in Chapter 6 .

In Chapter 5 time-varying copulas were used to study financial contagion. Contrary to the studies by Forbes and Rigobon (2002) and Rodriguez (2007) we relaxed the assumption that dependence changes simultaneously with volatility. Doing so allowed for new conclusions on how dependence changed during the Asian crisis. In particular it was found that the increase in correlation was 
significantly later than the increase in volatility and that in some cases higher volatility even seemed to decrease correlation. Overall we found strong evidence for contagion effects.

Chapter 6 introduced a new time-varying copula model, the stochastic copula autoregressive (SCAR) model. Issues related to estimation, inference, forecasting and possible extensions were discussed, and empirical applications both in-sample and out-of-samples were performed. Allowing dependence to evolve as a separate stochastic process worked very well as suggested by simulation results and an excellent data fit. Surprisingly, the model based on Gaussian copulas outperformed all other copulas.

Finally, Chapter 7 studied the tails of Gaussian and $t$-copulas with random correlations, motivated by the SCAR model. It was found that dependence in the tails is much larger for time-varying correlation models than for static ones with the same overall dependence. Furthermore, the penultimate coefficient of tail dependence is in general larger than its limit, the popular coefficient of tail dependence. The most intriguing result, however, was that Gaussian copula with random correlation are characterized by a new class of dependent tails that we label near asymptotic dependence. In this class the coefficient of tail dependence $\eta$ is equal to one, whereas the coefficient of tail dependence $\lambda$ is equal to zero ${ }^{1}$. This explains the good fit of the Gaussian SCAR model from Chapter 6 and makes this model more attractive than one initially would expect.

The results of this thesis suggest that copulas are a useful tool for modeling dependence, although sometimes care needs to be taken when interpreting results. For example, quite often the Gaussian copula is rejected in favor of 'more interesting' copulas, although we saw in this thesis that it is often just a matter of allowing for dynamic correlations. Also the concept of asymmetric dependence was, until now, mainly concerned with asymmetries in tail dependence, even though asymmetries at finite quantiles are much more relevant in practice. In general I find that the concept of tail dependence is sometimes abused in the copula literature by restricting the attention to models that allow for this feature. If I compare a Gaussian copula to e.g. a symmetrized Joe-Clayton copula the better fit of the latter model is not yet evidence of tail dependence. It may simply mean that an asymmetric model is closer to the true copula than a symmetric one. Relying on the concepts of quantile dependence or penultimate tail dependence may be much more appropriate in many situations.

Concerning time-varying copula models it is quite encouraging that different types of specifications are able to capture the dynamics of the dependence parameter quite well, which suggests that these models are quite robust. Nevertheless, it still remains to be seen what exactly drives changing

\footnotetext{
${ }^{1}$ Unfortunately, both $\eta$ and $\lambda$ are called coefficient of tail dependence in the literature.
} 
dependencies and whether conditionally Gaussian copulas are able to explain the observation of asymmetric dependencies in finite samples. The results in Chapters 3 and 6 suggest that this may indeed be the case, but more research needs to be done regarding this issue. Further important topics of future research are the specification and estimation of high dimensional time-varying copula models, time aggregation properties of dynamic correlation/dependence models, and the generalization of stochastic copula models to allow time trends, exogenous variables or correlated innovations for the dependence and volatility processes. 



\section{Bibliography}

Aas, K., C. Czado, A. Frigessi, and H. Bakken (2009). Pair-copula constructions of multiple dependence. Insurance: Mathematics and Economics 44, 182-198.

Abdous, B., A.-L. Fougères, and K. Ghoudi (2005). Extreme behaviour for bivariate elliptical distributions. The Canadian Journal of Statistics 33, 317-334.

Altissimo, F. and G. L. Violante (2001). The non-linear dynamics of output and unemployment in the u.s. Journal of Applied Econometrics 16, 461-486.

Amisano, G. and R. Casarin (2007). Particle filters for markov switching stochastic correlation models. In Proceedings of the 2007 Intermediate Conference "Risk and Prediction", Cleup, Padua, pp. 305-316.

Anderson, T. W. and D. A. Darling (1952). Asymptotic theory of certain goodness-of-fit criteria based on stochastic processes. Annals of Mathematical Statistics 23, 193-212.

Andrews, D. W. K. (1993). Tests for parameter instability and structural change with unknown change point. Econometrica 61, 821-856.

Ang, A. and G. Bekaert (2002). International asset allocation with regime shifts. Review of Financial Studies 15, 1137-1187.

Ang, A. and J. Chen (2002). Asymmetric correlations of equity portfolios. Journal of Financial Economics 63, 443-494.

Asai, M. and M. McAleer (2009). The structure of dynamic correlations in multivariate stochastic volatility models. Journal of Econometrics 150, 182-192. 
Asimit, A. V. and B. L. Jones (2007). Extreme behavior of bivariate elliptical distributions. Insurance: Mathematics and Economics 41, 53-61.

Bae, K.-H., G. A. Karolyi, and R. M. Stulz (2003). A new approach to measuring financial contagion. Review of Financial Studies 16, 717-763.

Baig, T. and I. Goldfajn (1998). Financial market contagion in the asian crisis. IMF Working Paper 98-155.

Bedford, T. and R. M. Cooke (2002). Vines - a new graphical model for dependent random variables. Annals of Statistics 30, 1031-1068.

Bollerslev, T. (1986). Generalized autoregressive conditional heteroskedasticity. Journal of Econometrics 7, 307-327.

Bonhomme, S. and J.-M. Robin (2006). Modeling individual earnings trajectories using copulas: France, 1990-2002. In H. Bunzel, C. B. J, G. R. Neumann, and J.-M. Robin (Eds.), Contributions to Economic Analysis, Vol. 275. "Structural Models of Wage and Employment Dynamics".

Bouyé, E., N. Gaussel, and M. Salmon (2001). Investigating dynamic dependence using copulae. Working paper, Financial Econometrics Research Centre, Sir John Cass Business School, London.

Breymann, W., A. Dias, and P. Embrechts (2003). Dependence structures for multivariate highfrequency data in finance. Quantitative Finance 3, 1-14.

Broto, C. and E. Ruiz (2004). Estimation methods for stochastic volatility models: A survey. Journal of Economic Surveys 18, 613-649.

Busetti, F. and A. Harvey (2008). When is a copula constant? a test for changing relationships. Cambridge Working Papers in Economics 0841.

Calvo, S. and C. M. Reinhart (1995). Capital inflows to latin america: Is there evidence of contagion effects? In A. Guillermo, S. Calvo, M. Goldstein, and E. Hochreiter (Eds.), Private Capital Flows to Emerging Markets After the Mexican Crisis. Institute for Internatioanl Economics, Washington, DC.

Candelon, B., A. Hecq, and W. Verschoor (2005). Measuring common cyclical features during financial turmoil: Evidence of interdependence not contagion. Journal of International Money and Finance 24, 1317-1334. 
Candelon, B. and H. Lütkepohl (2001). On the reliability of chow type tests for parameter constancy in multivariate dynamic models. Economics Letters 73, 155-160.

Candelon, B. and H. Manner (2007). Testing for asset market linkages: A new approach based on time-varying copulas. METEOR Research Memorandum RM/07/052, Maastricht University.

Candelon, B., J. Piplack, and S. Straetmans (2008). On measuring synchronization of bulls and bears: the case of east asian. Journal of Banking and Finance 32, 1022-1035.

Cappiello, L., R. Engle, and K. Sheppard (2007). Asymmetric dynamics in the correlations of global equity and and bond returns. Journal of Financial Econometrics 4, 537-572.

Cappiello, L., B. Gérard, and S. Manganelli (2005). Measuring comovements by regression quantiles. ECB Working Paper 501.

Carnero, M. A., D. Peña, and E. Ruiz (2004). Persistence and kurtosis in garch and stochastic volatility models. Journal of Financial Econometrics 2, 319-342.

Chen, X. and Y. Fan (2005). Pseudo-likelihood ratio tests for semiparametric multivariate copula model selection. The Canadian Journal of Statistics 33, 389-414.

Chen, X. and Y. Fan (2006a). Estimation and model selection of semiparametric copula-based multivariate dynamic models under copula misspecification. Journal of Econometrics 135, 125154.

Chen, X. and Y. Fan (2006b). Estimation of copula-based semiparametric time series models. Journal of Econometrics 135, 307-335.

Chen, X., Y. Fan, and A. Patton (2008). Simple tests for models of dependence betweeen multiple financial time series: with applications to u.s, equity returns and exchange rates. London Economics Financial Markets Group Working Paper 483.

Cherubini, G., E. Luciano, and W. Vecchiato (2004). Copula Methods in Finance. John Wiley and Sons Ltd.

Chollete, L., A. Heinen, and A. Valdesogo (2009). Modeling international financial returns with a multivariate regime switching copula. Journal of Financial Econometrics 7, 437-480.

Clark, P. K. (1973). A subordinate stochastic process model with finite variance for speculative prices. Econometrica 41, 135-155. 
Coles, S., J. Heffernan, and J. Tawn (1999). Dependence measures for extreme value analyses. Extremes 2, 339-365.

Corsetti, G., M. Pericoli, and M. Sbracia (2005). Some contagion, some interdependence: More pitfalls in tests of financial contagion. Journal of International Money and Finance 24, 1177-1199.

Creal, D., S. J. Koopman, and S. J. Lucas (2008). A general framework for observation driven time-varying parameter models. Tinbergen Institute Discussion Paper 08-108/4.

Danielsson, J. (1998). Multivariate stochastic volatility models: Estimation and comparison with vgarch models. Journal of Empirical Finance 5, 155-173.

Danielsson, J. and J. F. Richard (1993). Accelerated gaussian importance sampler with application to dynamic latent variable models. Journal of Applied Econometrics 8, 153.

de Matteis, R. (2001). Fitting copulas to data. Diploma thesis, University Zürich.

Demarta, S. and A. J. McNeil (2005). The t copula and related copulas. International Statistical Review 73, 111-129.

Dias, A. and P. Embrechts (2004). Change-point analysis for dependence structures in finance and insurance. In G. Szegoe (Ed.), Risk Measures for the 21st Century, pp. 321-335. Wiley Finance Series.

Diebold, F. X., T. A. Gunther, and A. S. Tay (1998). Evaluating density forecasts. International Economic Review 39, 863-883.

Dobric, J. and F. Schmid (2007). A goodness of fit test for copulas based on rosenblatt's transformation. Computational Statistics and Data Analysis 51, 4633-4642.

Doucet, A., N. de Freitas, and N. Gordon (2001). Sequential Monte Carlo Methods in Practice. Springer-Verlag, New York.

Dungey, M., R. Fry, and V. Martin (2006). Correlation, contagion and asian evidence. Asian Economic Papers 5, 32-72.

Dungey, M. and D. Zhumabekova (2001). Testing for contagion using correlation: Some words of caution. Pacific Basin Working Paper Series PB0109, Federal Reserve Bank of San Francisco. 
Durbin, J. and S. J. Koopman (2000). Time series analysis of non-gaussian observations based on state space models from both classical and bayesian perspectives (with disussion). Journal of the Royal Statistical Society, Series B 62, 3-56.

Durbin, J. and S. J. Koopman (2001). Time Series Analysis by State Space Models. Oxford University Press, New York.

Embrechts, P. (2009). Copulas: A personal view. Journal of Risk and Insurance 76, 639-650.

Embrechts, P., A. Höing, and A. Juri (2003). Using copulae to bound value-at-risk for functions of dependent risks. Finance and Stochastics 7, 145-167.

Embrechts, P., F. Lindskog, and A. McNeil (2003). Modelling dependence with copulas and applications to risk management. In Handbook of Heavy Tailed Distributions in Finance, Elsevier, pp. 329-384.

Embrechts, P., A. McNeil, and D. Straumann (2002). Dependence properties in risk management: Properties and pitfalls. In M. Dempster (Ed.), Risk Management: Value at Risk and Beyond, Cambridge University Press.

Engle, R. F. (1982). Autoregressive conditional heteroskedasticity with estimates of the variance of u.k. inflation. Econometrica 50, 987-1008.

Engle, R. F. (2002). Dynamic conditional correlation: A simple class of multivariate generalized autoregressive conditional heteroskedasticity models. Journal of Business and Economic Statistics 20, 339-350.

Engle, R. F. and B. Kelly (2008). Dynamic equicorrelation. Technical report, Stern Business School.

Engle, R. F. and S. Manganelli (2004). CAViaR: Conditional autoregressive value at risk by regression quantiles. Journal of Business and Economic Statistics 22, 367-381.

Engle, R. F. and K. Sheppard (2001). Theoretical and empirical properties of dynamic conditional correlation multivariate garch. NBER Working Paper 8554, National Bureau of Economic Research.

Erb, C. B., C. R. Harvey, and T. E. Viskante (1994). Forecasting international equity correlations. Financial Analysts Journal 50, 32-45.

Fan, J., M. Farmen, and I. Gijbels (1998). Local maximum likelihood estimation and inference. Journal of the Royal Statistical Society B 60,591-608. 
Fermanian, J.-D. (2005). Goodness of fit tests for copulas. Journal of Multivariate Analysis 95, $119-152$.

Fermanian, J.-D. and O. Scaillet (2003). Nonparametric estimation of copulas for time series. Journal of Risk 5, 25-54.

Fleming, J., C. Kirby, and B. Ostdiek (2001). The economic value of volatility timing. The Journal of Finance 56, 329-352.

Forbes, K. J. and R. Rigobon (2002). No contagion, only interdependence: Measuring stock market co-movements. The Journal of Finance 57, 2223-2261.

Frahm, G., M. Junker, and R. Schmidt (2005). Estimating the tail-dependence coefficient: Properties and pitfalls. Insurance: Mathematics and Economics 37, 80-100.

Garcia, R. and G. Tsafack (2008). Dependence structure and extreme comovements in international equity and bond markets with portfolio diversification effects. Working paper, EDHEC Risk Asset Management Research Centre.

Genest, C. and A.-C. Favre (2007). Everything you always wanted to know about copula modeling but were afraid to ask. Journal of Hydrologic Engineering 12, 347-368.

Genest, C., K. Ghoudi, and L.-P. Rivest (1995). A semiparametric estimation procedure of dependence parameters in multivariate families of distributions. Biometrika 82, 543-552.

Genest, C., J.-F. Quessy, and B. Rémillard (2006). Goodness-of-fit procedures for copula models based on the probability integral transformation. Scandinavian Journal of Statistics 33, 337-366.

Genest, C., B. Rémillard, and D. Beaudoin (2009). Goodness-of-fit tests for copulas: A review and a power study. Insurance: Mathematics and Economics 44, 199-213.

Genest, C. and L.-P. Rivest (1993). Inference procedures for bivariate archimedean copulas. Journal of the American Statistical Association 88, 1034-1043.

Giacomini, E., W. Härdle, and V. Spokoiny (2009). Inhomogeneous dependency modelling with time varying copulae. Journal of Business and Economic Statistics 27, 224Ü234.

Gombay, E. and L. Horváth (1996). On the rate of approximations for maximum likelihood tests in change-point models. Journal of Multivariate Analysis 56, 120-152. 
Granger, C. W. J., T. Teräsvirta, and A. Patton (2006). Common factors in conditional distributions. Journal of Econometrics 132, 43-57.

Guégan, D. and J. Zhang (2009). Change analysis of dynamic copula for measuring dependence in multivariate financial data. Quantitative Finance, forthcoming.

Hafner, C. M. and H. Manner (2008). Dynamic stochastic copula models: Estimation, inference and applications. METEOR Research Memorandum RM/08/043, Maastricht University.

Hafner, C. M. and O. Reznikova (2008). Efficient estimation of a semiparametric dynamic copula model. Manuscript, Institute of Statistics, UCL.

Hafner, C. M., D. van Dijk, and P. H. Franses (2006). Semiparametric modelling of correlation dynamics. In T. Fomby and C. Hill (Eds.), Advances in Econometrics, Volume 20, Department of Economics, Louisiana State University, pp. 59-103.

Hamilton, J. (1994). Time Series Analysis. Princeton University Press.

Hansen, E. B. (1994). Autoregressive density estimation. International Economic Review 35, 705730.

Hartman, P., S. Straetmans, and C. de Vries (2004). Asset market linkages in crisis periods. Review of Economics and Statistics 86, 313-326.

Harvey, A. (2009). Tracking a changing copula. Journal of Empirical Finance, forthcoming.

Harvey, A. C., E. Ruiz, and N. Shephard (1994). Multivariate stochastic variance models. Review of Economic Studies 61, 247-264.

Hashorva, E. (2005). Extremes of asymptotically spherical and elliptical random vectors. Insurance: Mathematics and Economics 36, 285-302.

Hashorva, E. (2008). Tail asymptotic results for elliptical distributions. Insurance: Mathematics and Economics 43, 158-164.

Hashorva, E. (2009). Conditional limit results for type i polar distributions. Extremes 12, 239-263.

Heffernan, J. (2000). A directory of coefficients of tail dependence. Extremes 3, 279-290. 
Heinen, A. and A. Valdesogo (2008). Asymmetric capm dependence for large dimensions: The canonical vine autoregressive copula model. available at ssrn: http://ssrn.com/abstract=1297506, CORE.

Hong, Y., J. Tu, and G. Zhou (2007). Asymmetries in stock returns: Statistical tests and economic evaluation. Review of Financial Studies 20, 1547-1581.

Jacquier, E., N. G. Polson, and P. E. Rossi (1994). Bayesian analysis of stochastic volatitliy models (with discussion). Journal of Business and Economic Statistics 12, 371-389.

Joe, H. (1997). Multivariate Models and Dependence Concepts. Chapman \& Hall/CRC.

Joe, H. (2005). Asymptotic efficiency of the two-stage estimation method for copula-based models. Journal of Multivariate Analysis 94, 401-419.

Joe, H. and J. J. Xu (1996). The estimation method of inference functions for margins for multivariate models. Technical Report 166, Department of Statistics, University of British Colombia.

Jondeau, E. and M. Rockinger (2006). The copula-garch model of conditional dependencies: An international stock market application. Journal of International Money and Finance 25, 827-853.

Junker, M. and A. May (2005). Measurement of aggregate risk with copulas. Econometrics Journal 8, $428-454$.

Junker, M., A. Szimayer, and N. Wagner (2006). Nonlinear term structure dependence: Copula functions, empirics, and risk implications. Journal of Banking ES Finance 30, 1171-1199.

Kim, S., N. Shephard, and S. Chib (1998). Stochastic volatility: Likelihood inference and comparison with arch models. Review of Economic Studies 65, 361-393.

King, M. and S. Wadhwani (1990). Transmission of volatility between stock markets. Review of Financial Studies 3, 3-33.

Ledford, A. W. and J. A. Tawn (1996). Statistics for near independence in multivariate extreme values. Biometrika 86, 169-187.

Ledoit, O. and M. Wolf (2004). A well-conditioned estimator for large-dimensional covariance matrices. Journal of Multivariate Analysis 88, 365-411. 
Lee, S. B. and J. K. Kwang (1993). Does the october 1987 crash strengthen the comovements among national stock markets? Review of Financial Economics 3, 89-102.

Lee, T.-H. and X. Long (2009). Copula-based multivariate garch model with uncorrelated dependent errors. Journal of Econometrics 150, 207-218.

Li, D. X. (2000). On default correlation: A copula function approach. Journal of Fixed Income 9, $43-54$.

Liesenfeld, R. and J. F. Richard (2003). Univariate and multivariate stochastic volatility models: Estimation and diagnostics. Journal of Empirical Finance 10, 505-531.

Longin, F. and B. Solnik (1995). Is the correlation in international equity returns constant: 1960-1990. Journal of International Money and Finance 14, 3-26.

Longin, F. and B. Solnik (2001). Extreme correlation of international equity markets. Journal of Finance 56, 649-676.

Manner, H. (2007). Estimation and model selection of copulas with an application to exchange rates. METEOR Research Memorandum RM/07/056, Maastricht University.

Manner, H. (2010). Testing for asymmetric dependence. Studies in Nonlinear Dynamics \& Econometrics, forthcoming.

Manner, H. and O. Reznikova (2009). Time varying copulas: a survey. Discussion paper DP 0917, Institut de statistique, Université catholique de Louvain.

Manner, H. and J. Segers (2009). Tails of gaussian and $t$-copulas with random correlations. Meteor research memorandum, Maastricht University, forthcoming.

Masson, P. R. (1999). Contagion: Monsoonal effects, spillovers, and jumps between multiple equilibria. In P. Agénor, M. Miller, and D. Vines (Eds.), The Asian Crises: Causes, Contagion and Consequences. Cambridge University Press.

Mercurio, D. and V. Spokoiny (2004). Statistical inference for time-inhomogeneous volatility models. Annals of Statistics 32, 577-602.

Nelsen, R. B. (2006). An Introduction to Copulas. Springer. 
Newey, W. and K. West (1994). Automatic lag selection in covariance matrix estimation. Review of Economic Studies 61, 631-653.

Patton, A. (2004). On the out-of-sample importance of skewness and asymmetric dependence for asset allocation. Journal of Financial Econometrics 2, 130-168.

Patton, A. (2006a). Estimation of multivariate models for time series of possibly different lengths. Journal of Applied Econometrics 21, 147-173.

Patton, A. (2006b). Modelling asymmetric exchange rate dependence. International Economic Review $47,527-556$.

Patton, A. (2009). Copula-based models for financial time series. In T. G. Andersen, R. A. Davis, J.-P. Kreiss, and T. Mikosch (Eds.), Handbook of Financial Time Series. Springer Verlag.

Pelletier, D. (2006). Regime switching for dynamic correlations. Journal of Econometrics 131, 445473.

Pesaran, H. and A. Pick (2007). Econometric issues in the analysis of contagion. Journal of Economic Dynamics and Control 31, 1245-1277.

Potter, S. M. (1995). A nonlinear approach to us gnp. Journal of Applied Econometrics 10, 109-125.

Ramchand, L. and R. Susmel (1998). Volatility and cross correlation across major stock markets. Journal of Empirical Finance 5, 397-416.

Richard, J. F. and W. Zhang (2007). Efficient high-dimensional importance sampling. Journal of Econometrics 141, 1385-1411.

Rodriguez, J. C. (2007). Measuring financial contagion: A copula approach. Journal of Empirical Finance 14, 401-423.

Simonoff, J. S. (1996). Smoothing methods in statistics. Springer series in statistics.

Sklar, A. (1959). Fonctions de repartition à $n$ dimensions et leurs marges. Publications de l'Institut de Statistique de L'Université de Paris 8, 229-231.

Straetmans, S. (1997). Extreme financial returns and their comovements. PhD thesis 181, Tinbergen Institute.

Taylor, S. J. (1986). Modelling Financial Time Series. JohnWiley and Sons, Chichester. 
Tse, Y. K. and A. K. C. Tsui (2002). A multivariate garch model with time-varying correlations. Journal of Business and Economic Statistics 20, 351-362.

van den Goorbergh, R. W. J., C. Genest, and B. J. M. Werker (2005). Bivariate option pricing using dynamic copula models. Insurance: Mathematics and Economics 37, 101-114.

van der Vaart, A. W. (1998). Asymptotic Statistics. Cambridge University Press, New York.

van Dijk, D., T. Teräsvirta, and P. H. Franses (2002). Smooth transition autoregressive models - a survey of recent developments. Econometric Reviews 21, 1-47.

Vuong, Q. H. (1989). Likelihood ratio tests for model selection and non-nested hypothesis. Econometrica 57, 307-333.

Yu, J. and R. Meyer (2006). Multivariate stochastic volatility models: Bayesian estimation and model comparison. Econometric Reviews 25, 361-384. 



\section{Nederlandse samenvatting}

Dit proefschrift heeft ten doel het uitbreiden van bestaande én het ontwikkelen van nieuwe technieken om afhankelijkheid tussen twee of meerdere economische variabelen te meten en te modelleren. Aangezien dit een groot spectrum van potentiële technieken en toepassingen behelst concentreren wij ons op modellen voor afhankelijkheid die gebaseerd zijn op zogenaamde copula functies. De praktische toepassingen hiervan bestaan voornamelijk uit het modelleren van financiële tijdreeksen, zoals beurs- en/of wisselkoersen. Desalniettemin zijn veel van deze methodes toepasbaar op een breed scala aan economische en niet-economische variabelen. Veel onderzoek is reeds gedaan naar de toepassing van copulas binnen de financiële econometrie en veel problemen zijn opgelost, maar er zijn nog altijd een groot aantal belangrijke open kwesties.

Het belangrijkste onderwerp van dit proefschrift is het herkennen van veranderingen in de aard van de afhankelijkheid in de tijd en het onderzoeken wat deze veranderingen precies inhouden voor de modellering van afhankelijkheid met copulas. De veranderende afhankelijkheid kan vanuit verschillende perspectieven worden bekeken en in dit proefschrift worden er twee van besproken en onderzocht. De eerste invalshoek is die van onvoorwaardelijke afhankelijkheid, waar de afhankelijkheid voor grote en voor kleine waardes van de variabelen verschillend is. Dit is wat gewoonlijk asymmetrische afhankelijkheid wordt genoemd en vrij vaak voorkomt in financiële gegevens, wanneer de afhankelijkheid hoger is tijdens baissemarkten dan tijdens een hausse van de beurs. Bepaalde copulas zijn geschikt om deze onvoorwaardelijke asymmetrische afhankelijkheidsstructuren te modelleren. Dit is één van de belangrijkste redenen van de recente interesse in copulas voor financiële tijdreeksen. Het tweede en in het bijzonder het derde hoofdstuk behandelen technieken om het bestaan van dergelijke asymmetrische afhankelijkheidsstructuren aan te tonen, ervoor te toetsen en deze te modelleren. 
Het tweede onderwerp omvat copulas die naast asymmetrie ook van eigenschappen veranderen gedurende de tijd, een eigenschap die belangrijk is bij het bestuderen van tijdreeksen. Tijdsafhankelijke, of voorwaardelijk afhankelijke copulas zijn geïntroduceerd door Patton (2006). Gezien het feit dat de volatiliteit en de correlatie van financiële tijdreeksen vaak sterk variëren over tijd, is de aanname van tijdsonafhankelijkheid twijfelachtig. Eenvoudige technieken, zoals het meten van afhankelijkheid over sub-periodes of gebruik maken van een rollend venster, zullen vrijwel altijd aangeven dat de veronderstelling van een tijdsinvariante copula niet adequaat is. Desondanks is het onduidelijk hoe de variatie in de tijd van een afhankelijkheidsmaatstaven het beste gemodelleerd kan worden. Daarnaast is het herkennen door middel van testen en het voorspellen niet voor de hand liggend. Dit proefschrift geeft antwoorden op dergelijke vragen en stelt nieuwe methodes voor om tijdsafhankelijke copulas te gebruiken.

Hoofdstuk 2 dient als een inleiding naar copulas en de technieken die nodig zijn om met deze te werken. De formele definities van copulas en verwante concepten worden verstrekt. Dit wordt gevolgd door een discussie van de belangrijkste theoretische resultaten en de meest gangbare copula maatstaven van afhankelijkheid worden geïntroduceerd. Hierna worden de populairste parametrische copulas gepresenteerd, tezamen met een korte bespreking van hun eigenschappen en methodes om gegevens te simuleren. Daarnaast worden verschillende methodes om parameters van copulas te schatten toegelicht en wordt de belangrijke kwestie van modelselectie kort besproken. Ten slotte worden de verschillende copulas geschat voor een aantal wisselkoersreeksen van Latijns-Amerikaanse munten vis-à-vis de Amerikaanse dollar en de beste modellen voor deze reeksen worden geïdentificeerd.

Hoofdstuk 3 behandelt uitsluitend asymmetrische afhankelijkheid, dus situaties waar de afhankelijkheid verschillend is voor grote en kleine waarden van een variabele. Om dit te meten wordt een voorwaardelijke versie van Kendall's tau, een populaire rangcorrelatiecoëfficiënt, bepaald. Hierna worden de formules verstrekt om deze voor elke relevante copula te berekenen. Twee waarschijnlijkheidsratiotesten, die gebaseerd zijn op bekende copulas, worden voorgesteld om de (ongeldige) hypothese van symmetrische afhankelijkheid te testen. Deze nieuwe testen hebben betere grootte eigenschappen en een grotere nauwkeurigheid dan een concurrerende test die door Hong et al. (2007) wordt voorgesteld. Bovendien genereren wij drie voorbeelden van processen die tot asymmetrische afhankelijkheid leiden. Deze processen worden zowel analytisch als door simulaties bestudeerd. Tot slot illustreren wij het gebruik van onze testen bij beurskoersen, het driemaandelijkse Amerikaanse BNP en werkloosheidspercentage. Wij vinden bewijs voor asymmetrie en niet lineair gedrag van deze variabelen.

In hoofdstuk 4 herzien wij acht concurrerende specificaties voor tijdsafhankelijke copulas en be- 
spreken kort hoe deze worden geschat. Wij bespreken dan hoe de mate van aanpassing van een model kan worden vergeleken en getest in het geval van tijdsafhankelijke copulas. Een simulatiestudie vergelijkt de eigenschappen van alternatieve technieken om de onderliggende tijdsafhankelijke afhankelijkheidsparameter te schatten, waarbij deze parameter onder verschillende veronderstellingen wordt gegenereerd. Een empirische toepassing illustreert de eigenschappen van de verschillende methodes voor beurs- en wisselkoers reeksen die met verschillende waarnemingsfrequenties worden gemeten. Naast de evaluatie van de statistische aanpassing van de verschillende modellen vergelijken wij ook hun prestaties door middel van risicomaatstaven.

Hoofdstuk 5 behandelt het toetsen van financiële besmetting tijdens de Aziatische crisis met gebruik van copula technieken. Een nieuwe methode om te testen voor verhoging van de afhankelijkheid tussen beurzen na een financiële crisis wordt voorgesteld. Deze verhoogde afhankelijkheid staat ook bekend als verschuivingsbesmetting. Toegepast op de recente Aziatische crisis van 1997 bevestigt de analyse dat veranderingen in de variantie altijd die in de afhankelijkheidsparameter voorafgaan. Ondersteunend bewijs voor het plaatsvinden van verschuivingsbesmetting wordt geleverd door het vinden van een significante "J-vorm" in de evolutie van de afhankelijkheidsparameter.

In hoofdstuk 6 wordt een nieuw tijdsafhankelijk copula model geïntroduceerd, namelijk het stochastische copula autoregressieve (SCAR) model. Diverse aspecten van dit model zoals schatten, toetsen en voorspellen worden besproken. Door middel van Monte Carlo simulaties en twee empirische toepassingen, met zowel voorspelling binnen als buiten de steekproef, wordt dit model vergeleken met het DCC GARCH model van Engle (2002) en het dynamische copula model van Patton (2006).

Hoofdstuk 7 bestudeert het staartgedrag van Gaussische en $t$-copulas wanneer de correlatieparameter wordt gegenereerd door een onbekend willekeurig proces. Wij vinden dat zowel de voorlaatste als asymptotische staartafhankelijkheid veel groter is voor elliptische copulas met willekeurige correlaties, dan voor statische met dezelfde onvoorwaardelijke correlatie. Voorts tonen wij aan dat voor Gaussische en Student copulas de voorlaatste coëfficiënt van staartafhankelijkheid over het algemeen groter is dan zijn grens, de coëfficiënt van staartafhankelijkheid. Tot slot, voor Gaussische copulas verkrijgt men, wanneer men mengt over de correlatie parameter rho, een copula waarvan staarten asymptotisch onafhankelijk zijn, maar die tegelijkertijd in een nieuwe klasse van asymptotische afhankelijkheid vallen, die wij als "dichtbij asymptotische afhankelijkheid" etiketteren, betekenend dat bij praktisch relevante kwantielen de staarten afhankelijk zijn. Wij verstrekken verscheidene numerieke illustraties van onze theoretische bevindingen en bespreken de gevolgen voor modellering 
door een simulatiestudie én een illustratie uit te voeren met behulp van financiële tijdreeksen. 


\section{Curriculum Vitae}

Hans Manner was born on October 30, 1980 in Frankfurt/Oder, Eastern Germany. In January 1989, shortly before the fall of the Berlin wall, he migrated with his family to Western Germany. In 1997/1998 he spent a year studying at a US High School in Sodus, New York, earning an honorary High School Diploma. In 2000 he received his Abitur from the Gymnasium Große Schule. He did his Civil Service at a retirement home in Dresden, before studying Econometrics at Maastricht University from 2001 to 2005. He spent a semester in Lima, Peru in the fall of 2004 and obtained his Master's Degree in Econometrics in July 2005. From November to December 2005 he did an internship at the Peruvian Central Reserve Bank. He joined the Department of Quantitative Economics at Maastricht University in April 2006 and worked under the supervision of Prof. dr. Franz C. Palm and Prof. dr. Jean-Pierre Urbain. Starting in April 2010 Hans will be working as a Junior Professor in Econometrics at the University of Cologne. 
